\title{
A MODEL-THEORETIC REALIST INTERPRETATION OF SCIENCE
}

\author{
by
}

EMMA BERNET RUTTKAMP

submitted in accordance with the requirements

for the degree of

DOCTOR OF LITERATURE AND PHILOSOPHY

in the subject

PHILOSOPHY

at the

UNIVERSITY OF SOUTH AFRICA

PROMOTER: PROF J HEIDEMA

JOINT PROMOTER: PROF W KISTNER

NOVEMBER 1998 
Student number: 3153-783-9

\section{I declare that}

A model-theoretic realist interpretation of science is my own work and that all the sources that I have used or quoted have been indicated and acknowledged by means of complete references.

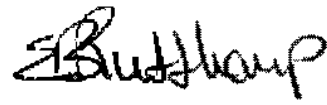

27 November 1998

Ms EB Ruttkamp

Date 


\begin{abstract}
My model-theoretic realist account of science places linguistic systems and the corresponding non-linguistic structures at different stages of the scientific process. It is shown that science and its progress cannot be analysed in terms of only one of these strata. Philosophy of science literature offers mainly two approaches to the structure of scientific knowledge analysed in terms of theories and their models, the "statement" and the "non-statement" approaches. In opposition to the statement approach's belief that scientific knowledge is embodied in theories (formulated in some (first-order) symbolic language) with direct interpretative links - via so-called "bridge principles" - to reality, the defenders of the non-statement approach believe in an analysis where the language in which the theory is formulated plays a much smaller role than the (mathematical) structures which satisfy that theory.

The model-theoretic realism expounded here retains the notion of a scientific theory as a (deductively closed) set of sentences, while simultaneously emphasising the interpretative role of the conceptual (i.a. mathematical) models of these theories. My criticism against the non-statement approach is based on the fact that merely "giving" the theory "in terms of" its mathematical structures leaves out any real interpretation of the nature and role of general terms in science. Against the statement approach's "direct" linking of general theoretical terms to reality, my approach interpolates models between theories and (aspects of) reality in the interpretative chain.

The links between the general terms of scientific theories and their interpretations in the various models of the theory regulate the whole referential process. The terms of a theory are "general" in the sense that they are the result of certain abstractive conceptualisations of the object of scientific investigation and subsequent linguistic formulations of these conceptualisations. Their (particular) meanings can be "given back" only by interpreting them in the limited context of the various conceptual models of their theory and, finally, by finding an isomorphic relation between some substructure of the conceptual model in question and some empirical conceptualisation (model) of relevant experimental data. In this sense the notion of scientific "truth" becomes inextricably linked with that of articulaled reference, as it - given its model-dependent nature - should be.
\end{abstract}

Key terms: science; theory; model; interpretation; reference; truth; reality 


\section{CONTENTS}

Chapter One: Introduction 1

1.1 The statement and non-statement accounts of science 1

1.2 The interpretation and use of the notion of "model" in

philosophy of science 5

1.2.1 Introduction

1.2.2 Heuristic uses of the notion of "model"

1.2.3 Variations on non-statement uses of the notion of "model" 11

1.2.4 The implications for philosophy of science of the use of the notion "model" in econometrics 14

Chapter Two: A model-theoretic account of sclence

2.1 Introduction 18

$\begin{array}{lll}2.2 & \text { Terminological note } & 21\end{array}$

2.3 The formulation of scientific theories 23

2.4 The interpretation of scientific theories 29

2.5 The process of science 36

2.5.1 Introduction 36

2.5.2 The nature of scientific progress 36

$\begin{array}{lll}\text { 2.5.3 Paradigms and models } & 42\end{array}$

2.5.3.1 Paradigms as constellations of group commitments $\quad 42$

2.5.3.2 Paradigms as shared examples $\quad 44$

$\begin{array}{lll}\text { 2.5.4 Conclusion } & 46\end{array}$ 
Chapter Three: Variations of the non-statement view of science

3.1 Introduction

3.2 Patrick Suppes's set-theoretical approach to science

3.3 The structuralist programme

3.4 The semantic approaches of Beth, Van Fraassen, and Suppe 76

3.5 Ronald Giere's naturalistic approach to science 82

3.6 Wójcicki's empiricist semantics of science 85

$\begin{array}{lll}3.7 & \text { Conclusion } & 88\end{array}$

Chapter Four: Nancy Cartwright and the lying laws of physics 94

$\begin{array}{lll}4.1 & \text { Introduction } & 94\end{array}$

4.2 Phenomenological and fundamental laws 94

4.3 The role of models in science and Cartwright's "simulacrum" $\begin{array}{ll}\text { account of science } & 109\end{array}$

$\begin{array}{ll}\text { 4.4 The process of science revisited } & 127\end{array}$

4.5 The "abstract" and the "concrete" 130

4.6 Nature's capacities causally explained 134

4.7 The "abstract" and the "concrete" revisited 143

$\begin{array}{lll}4.8 & \text { Conclusion } & 150\end{array}$ 
Chapter Five: A mode/-theoretic realism 152

5.1 Introduction 152

$\begin{array}{lll}5.2 & \text { Reality and science } & 154\end{array}$

5.3 A modified image of science 160

5.3.1 The distinction between observation and theory 160

$\begin{array}{ll}\text { 5.3.2 Science is cumulative } & 161\end{array}$

5.3.3 The deductive structure of science 162

$\begin{array}{ll}\text { 5.3.4 Scientific terminology } & 162\end{array}$

5.3.5 The contexts of justification and discovery 163

5.4 The empirical interpretations of scientific theories 164

$\begin{array}{ll}\text { 5.4.1 Introduction } & 164\end{array}$

5.4.2 Empinical adequacy 164

$\begin{array}{lll}\text { 5.4.3 Transduction } & 170\end{array}$

5.5 The succession of theories, verisimilitude, underdetermination, and other intertheoretic issues $\quad 173$

$\begin{array}{lll}5.6 & \text { Conclusion } & 186\end{array}$

$\begin{array}{lll}\text { Chapter Six: } & \text { Conc/usion } & 189\end{array}$

6.1 The meaning of a model-theoretic realism for philosophy of $\begin{array}{ll}\text { science } & 189\end{array}$

$\begin{array}{lll}6.2 & \text { Historical note } & 192\end{array}$

Endnotes 


\section{PREFACE}

On completion of an endeavour such as this, there are always numerous people to thank.

To my supervisor Professor Johannes Heidema - who has also become a treasured friend - I express my profound gratitude for guiding my often very faltering thoughts with endless patience and understanding.

I also wish to thank my co-supervisor Professor W. Kistner for the time and effort she has put into assisting the completion of this thesis.

On a more personal note, I thank my husband for his encouragement, my son for showering me with uncritical love whenever I needed it, and my close family and friends for all the various roles they have played in helping me realise my goal. 


\section{CHAPTER ONE: INTRODUCTION}

\subsection{The statement and non-statement accounts of science}

In this thesis I shall offer a model-theoretic realist interpretation of the processes and products of (natural) science built on recent work in the philosophy of science dedicated to analysing the natural sciences in terms of conceptual (mathematical) models of theories and the various semantic relations between such models, theories, and (aspects of) reality. Although analyses of theory-reality and model-reality links have long been a part of philosophy of science, I shall concentrate on the more recent developments concerning these issues in a model-theoretic context. Such analyses touch on core questions of the philosophy of science, such as questions regarding the nature of scientific theories, the usual realist inspired questions about the possibility and character of relations between scientific theories and reality, the notion of scientific truth, and, in general, on the nature of scientific progress. A model-theoretic realism offers, via analyses of the structure of scientific theories and the processes of science, a scientific realism that needs no metaphysical substructure as justification. This particular kind of realism is a very common sense kind of realism in the sense that rather than trying to justify everyday features of the science-reality relationship such as the underdetermination of scientific theories by their data - it not only acknowledges these features, but uses them to strengthen its realist claims. The underlying aim of this thesis is to show that claiming - as a model-theoretic realism does - that reality exists "outside" of human practice, neither means that reality is unknowable nor, at the other extreme of the scale, that science simply mirrors it. By analysing the structure of scientific theories model-theoretically (Chapter 2), it will be shown (Chapter 5) Ihat a model-1heoretic approach to science and its processes and products offers the best kind of scientific realism - i.e. a scientific realism with as little metaphysical content as possible.

First a few words on the notion of "science" as it will be used in this thesis. In principle, my model of science is applicable to all sciences (natural, behavioural, human, social, economic, and so on). I shall in this thesis however put the emphasis almost exclusively on the natural sciences, and in particular on physics, for the following reason: all of the three 
main aspects of my model (or interpretation) of science (namely empirical models, conceptual models, and theories) are - mostly - more simple and more clearly delineated in the case of the natural sciences (and in physics in particular) than in the other sciences. In Section $1.2,4$ I briefly discuss the use of the notion of "model" in econometrics to illustrate the applicability of a model-theoretic approach outside the natural sciences. See more on the use of terminology in this thesis in Section 2.2.

The use of the notion of models is nothing new in either philosophy of science or the (empirical) sciences themselves. Writers such an Achinstein (1968), Hesse (1963), and, more recently, Redhead (1980) have paid much attention to the heuristic uses of models in the development of scientific theories. In his article, entitled $A$ companson of the meaning and uses of models in mathematics and the empincal sciences, Patrick Suppes (1960) reviews the various uses of the notion in mathematical statistics, psychology, economics, and physics. Nancy Cartwright $(1989,1995 a, 1995 b)$ also often makes use of the analogies between the ways in which models are used in econometrics and theoretical physics to illustrate her views of the nature of scientific theones. In Section 1.2 I shall give a brief overview of some of the different interpretations and uses of the notion of "model" in philosophy of science.

In current philosophy of science, the most interesting questions centre around the ways in which writers distinguish between and assign roles to theories and the mathematical structures that interpret them and in which they are true, l.e. between scientific theories as linguistic systems and their non-linguistic models. In this context I shall in Chapter 2 set out my own model-theoretic account of the processes and products of science.

Philosophy of science literature offers us mainly two approaches to the structure of the products of science analysed in terms of linguistic and non-linguistic systems, the "statement" or syntactic approach, and the "non-statement" or semantic approach. The statement approach is characteristic of philosophers and logicians like Carnap, Hempel, and Nagel. The advocates of this approach use the tools of mathematical logic to depict theories as axiomatic systems in some well-defined language, and study the syntax and semantics of theories via the proof and model theories of language. The advocates of the non-statement 
view of science's products emphasise the tools of algebra and set theory. This approach originated with Poincerés work ìn geometry and mechanics and has started developing through semantic analyses of non-stalement reconstructions of certain scientific theories done by Von Neumann (1955), Adams (1959) and Suppes (see McKinsey, Sugar \& Suppes (1953), Suppes (1959)), and also Montague (1962).

The statement or "received" approach depicts the rational reconstruction of the language of science as a syntactic system with an axiomatised deductive theory formulated within that system. Its defenders usually charactenise theories in terms of two parts. First they identify an abstract formal calculus (a symbolic language) in which the primitive symbols (which in this case are terms that do not have obvious relations with "observation" terms, i.e. so-called "theoretical" terms fike "electron", "particle", "mass", and so on) of the theory are set out. The second part of the structure of a scientific theory they depict as a set of rules (called "correspondence rules" by Camap ${ }^{2}$ and "bridge principles" by Hempel) that assigns empirical (observational) content to the logical calculus by providing "co-ordinating definitions" or "empinical interpretations" for at least some of the primitive and defined symbols of the calculus, and in that way - supposedly - establishes direct links between elements of the theory and elements of reality.

Advocates of the "non-statement" or semantic approach view the rational reconstruction of the language of science in terms of a syntactic system and a family of interpretations (or models) of that syntax. In opposition to the statement approach's belief that theories are formulated in some (first-order) symbolic language with direct links to reality, the defenders of the non-statement approach believe in an analysis where the language in which the theory is formulated plays a much smaller role. They hold that foundational problems in the various sciences can in general be better addressed by focussing on the models these sciences employ than by reformulating the products of these sciences in some appropriate language.

The non-statement approach has had several branchings since Patrick Suppes has emphasised - against the metamathematical musings of the advocates of the received view 
- the clarifying advantages of set-theoretical reconstructions of empirical theories. I shall discuss Suppes's approach in section 3.2. The structuralist programme led by Sneed, Stegmüller, Moulines, and Balzer offers a structural analysis of science, and I shall discuss this programme in section $3.3^{3}$ The semantic approach offers an examination of the content of theories via Beth's notion of state-spaces and is supported by Van Fraassen, Suppe, and the (naturalistic) view of science offered by Ronald Giere. These notions are discussed in sections 3.4 and 3.5. Finally, I shall discuss the approach offered by a kind of affiliation to the semantic approach, which is headed by Wójcicki, and Przelewski ${ }^{4}$ - who both concentrate on offering an empinicist semantics for science while also working on the problem of "analyticity" - and also followed by Tuomela and Rantala", who apply this approach respectively to problems conceming the nature of theoretical terms and the problems of definability and indefinability in science. Wójcicki's approach is briefly discussed in section 3.6 .

The choice between the statement and non-statement approaches seems trivial as far as theories formulated in first-order languages are concerned. As Theo Kuipers (Kuokkanen, 1994:5) points out, the set of models (that is, structures for which the statements of the theory are true) of theories formulated as a set of statements of some first-order language is exactly the kind of (set of) structures that the defenders of the non-statement approach view as the building blocks of empirical theories. In other words (ibid.), possible interrelations between the two approaches exist in so far as an axiomatised theory may be characterised by a class of interpretations which satisfy it, and an interpretation (or class of interpretations) may be characterised by a set of sentences which it satisfies (and in neither case will the characterisation be unique $)^{7}$. I believe though that clarification of certain core problems in philosophy of science - such as the relations between scientific theories and reality and the notion of scientific truth - is more likely when following the emphasis on models that the semantic approach offers, while retaining the statement view's analysis of a theory as a (deductively closed) set of sentences. I thus do not go along with either of the two approaches. In the empirical sciences no theoretical entity is ever such a "free creation" of 
the human mind that its possible links with reality may be discarded in an examination of the various truth relations in which the theory (albeit via its models) stands during the various stages of the scientific process. I shall set out my own approach in Chapter 2, before continuing to discuss - in Chapter 3 - some of the main views in the non-statement school of thought, using my approach as a unifying meta-view.

In Chapter 4 I shall, within the context of my model-theoretic account, review the troubled attitude of Nancy Cartwright towards the "fundamental laws" of science, i.e. the axioms of the deductive set of sentences I refer to as a scientific theory. Cartwright's work is important in my terms since she is currently probably the most influential philosopher of science - writing on the issue of the realism of scientific theories - who does not subscribe to either the statement or the non-statement approaches to science. I do not think that she succeeds either in reconciling or superseding these two approaches though. A modeltheoretic account however solves a remarkable number of statement as well as nonstatement problems and so supersedes these approaches, reconciling their best features. Cartwright pays much attention to general realist issues (think of her pre-occupation with the so-called "lying" laws of physics). In Chapter 5, I too, shall concentrate on realist issues and offer a model-theoretic interpretation of scientific realism, before concluding on the menits of a model-theoretic realist interpretation of science in Chapter 6.

However, let us first examine the meaning and use of the notion of model in philosophy of science.

\subsection{The interpretation and use of the notion of "model" in philosophy of science}

\subsubsection{Introduction}

One intuitive idea of a model is a possible realisation in which a theory is satisfied in the Tarskian sense ${ }^{8}$. Suppes (1960: 290) points out that the most important distinguishing feature of the relation between a theory and a model of a theory is that a theory is a linguistic entity consisting of a set of sentences, and models are non-linguistic entities in which the theory is satisfied. The notion of "model" is used in widely different ways by philosophers of 
science and scientists alike. However, if one examines these various interpretations and uses a little more closely, it is possible to find common features in the ways in which the notion of a model is applied that show the advantages of this notion in terms of clarifying the intricacies of the processes and products of science. Suppes $(1960: 289,290)$ remarks rightly that the concept of "model" in the sense of Tarski may be used without distortion and as a fundamental concept in any discipline. In this sense he claims that the meaning of the concept of model in mathematics is the same as in - or very close to that of - the empirical sciences, and finds the difference rather in the various uses of the concept, in the sense that "mathematicians ask a certain kind of question about models and empirical scientists tend to ask another kind of question" (ibid.) ${ }^{8}$. However, rather than perhaps obscuring the role of models in rational reconstructions of science, these different kinds of "questions" that may be asked about models merely illustrate and strengthen the validity of using the notion of "model" in these kinds of rational reconstruction.

Suppes (in Morgenbesser, 1967: 57) apparently agrees, although perhaps with certain reservations, since he remarks that "[q]uite apart from questions about direct empinical observations, it is pertinent and natural from a logical standpoint to talk about the models of a theory ... [since these] models are highly abstract, non-linguistic entities, often quite remote in their conception from empirical observations". Therefore, he acknowledges the apparent logical problem undertying questions concerning that which the concept of a model actually has to add to the usual discussions of empirical interpretations of theories. He comments (ibid.) that mostly philosophers find it easier to talk about theories than about models of theories, because mostly, their examples are simple in nature and can be discussed in purely linguistic (first-order logic) terms, while introducing the notion of the model(s) of a theory adds a higher mathematical element to the discussion. However he offers the main part of the answer to these questions himself when he (in Morgenbesser, 1967: 58) points out that dealing with more complicated theories, like quantum mechanics, and classical thermodynamics, we are not always involved with "simple" examples at all. In these cases he claims (ibid.) we also need the general results of set theory as well as many results 
conceming the real numbers, since direct formalisation of such theories in first-order logic is rather impractical, given that the degree of complexity of theories of this sort is similar to that of theories studied in pure mathematics. He writes (ibid.): "In such contexts it is very much simpler to assert things about models of the theory rather than talk directly and explicitly about the sentences of the theory, perhaps the main reason for this being that the nolion of a sentence of the theory is not well-defined when the theory is not given in standard formalisation". Also, some calculations are simply too complex to do in any other context than in that of some model of the theory in question - think for example of a leaf blowing in the wind. To predict where it will come to rest, i.e. to describe its movement, it is possible to use Newton's laws of motion and his law of gravitation ${ }^{10}$, However, the actual calculations would be really complex, and therefore it would be more practical to work in some model of his "theory" that focuses only on certain of the aspects of the real system.

\subsubsection{Heuristic uses of the notion of "model"}

Redhead (1980: 146) comments that early depictions of "theoretical models", such as Achinstein's (1968), almost always implied these models to be false. The reason for this is that mostly, at that stage ${ }^{11}$, models were used in the heuristic sense of suggesting new ways to look at certain problems and the notion of "theorelical model" was generally explained in terms of a set of assumptions about some system, attributing some kind of inner structure to the system, and thus being a "simplified approximation" (Redhead, 1980: 146) of the system modelled. Also, these models were used - and are of course, still used for these reasons - to justify the production of predictive theories - i.e. theories formulated to explain one group of phenomena (one kind of real system) which then prove to have the ability to predict events concerning another type of system.

Thus, after the positivist reign, most of the initial philosophical reflection concerning models was concemed with the role of models within some kind of "logic of analogy". In these cases it is obvious that models will tum out to be "false" of the system in reality on which the development of the theory in question is focussed, since they are not interpreted in terms of 
mathematical models (and their empirical substructures) of the theories concerned. This usage of the notion of model should however not be confused with a Tarskian interpretation of the notion in the sense of models "making" the theories they interpret "true", ${ }^{12}$

A related way in which the notion of "model" has also entered into philosophy of science debates is via the well-known use of "iconic" models by Hesse (1963), Achinstein (1968), and Redhead (1980). Both Nagel (1961) and Hesse (1963) view the empirical interpretation of a scientific theory in terms of mathematical and iconic models. Achinstein (1968) defines an iconic model as a set of simplified and approximate assumplions about some system. These assumptions attribute a certain inner structure to the system and are proposed within the context of some more "basic" theory. Hesse (1963: 19) sees these madels as also possibly exhibiting analogies between the system modelled and some other system. Da Costa and French (1990: 258) explain these notions in terms of the example of the billiard ball model of kinetic gas theory. Viewed as an iconic model, it offers an image or representation (picture) of a system of gas atoms. There also exists positive analogy in Hesse's terms since there is a similarity between certain aspects of the system itself and certain aspects of the model. The billiard ball model is, however, also a mathematical model of the kinetic theory of gases, in the sense that a semantic interpretation is given to the theory in terms of the billiard ball system such that the axioms of the theory are true under that interpretation. $^{13}$

The main problem with this kind of approach is, as Da Costa and French (1990: 258) point out, a realist one. The mathematical model is supposed to somehow say something about how the world is (offer an empirical interpretation of the theory), while the same is not expected from an iconic model. Van Fraassen, for instance, ignores these kinds of problem by arguing that scientific theories are indeed nothing more but iconic models of phenomena (observable objects). Da Costa and French (1990: 259) go on to claim that on the "realist view" it seems that the difference between a theory and models of the theory is that the theory may be true and its models can only be false. This is the same kind of explication that Redhead (1980) offers. The problem is that these kinds of approach view the role of models 
purely in instrumental terms, that is, in terms of clarifying the axioms of the theory, or extending the domain of a theory, or perhaps even in limiting the domain of theory.

A model-theoretic realist, however, I claim, understands that the role of models in the scientific process is far more important than that. Such a philosopher of science acknowledges that the use of the notion of model in the Tarskian sense as a mathematical interpreting structure is by far the most important one as far as discovering possible links with reality is concerned, and thus such a philosopher only speaks of theories being true in particular models, and not of either theories or models being true or false per se, in the literal absolute sense of the word. ${ }^{14}$ Do not take me wrong, the powerful role of these kinds of (iconic) models and also of models as "approximations" to theories - Redhead's (1980: 147) "impoverishments" of theories ${ }^{15}$ - in e!aborating the domains of scientific theories, cannot be ignored. ${ }^{16}$ However, it should be noted that this is a different use of the term than that of the term as a mathematical model, and also that mathematical models can indeed also be used with these kinds of aim in mind.

Redhead (1980: 162) makes an interesting remark about the role of models in science, that reminds much of Cartwright's (1983) kind of approach. He (1980: 162) writes [i]f we always tried to solve every problem with absolute accuracy, and neglecting no 'accidental' aspects, science would never get started. Science depends on the possibility of ignoring accidents, of isolating certain key features in a situation. These are captured by models, although in the very act of idealisation or approximation we convince ourselves that the model is indeed false. It is moreover in respect of modelling that the imaginative and intuitive element in theoretical physics is most clearly seen ... Modelling is certainly an art, involving a number of logical gaps ....

Of course mathematical models are also idealisations of some real system. The problem that I have with Nancy Cartwright's kind of approach, and therefore with this kind of remark, is however that the conceptual models of some theory, despite their potentially idealised nature, may still be found to be related to some real system(s). I argue that it is not "truth" in terms 
of so-called "false" (or "distorting") idealisations of real systems that philosophers of science should be concemed with at all, but that rather they should concentrate on the Tarskian notion of truth in terms of satisfaction. This kind of (satisfaction) relation establishes (semantic) links between some theory and the models of the theory. It also may determine relations of empincal adequacy in terms of a modified version of Tarskian satisfaction established by (possible) links between the models of the theory and some system(s) in reality. See Chapters 2,4 , and 5 for more on this issue.

Redhead (1980: 147) also discusses the notion of "enniching models". This kind of model is more interesting for my purposes, since it seems to play a role very close to that which I ascribe to the "initial" or "intended conceptual" models in my analysis of the process of science. ${ }^{17}$ Redhead claims (ibid.) that these enriching models may come into scientific play whenever a theory seems to be "incompletely specified" such that "considerable latitude" is allowed in the selection of the detailed structure of the theory. He (ibid.) offers the example of axiomatic field theory where any number of fields may possibly satisfy the axioms of the theory, and claims that an "enriching model" may then be introduced to fill in "missing detail". Obviously here the model is not believed to be contradicting the theory, since no "completed" theory has yet been formulated. However, as soon as the model "resembles an exact theory" it is called a "theory" and not a "model" any longer."

Another more heuristically related use of the notion of "model" is the application of the notion in the construction of Gedanken experiments - usually done in the empirical sciences. Here, again, I see this as mainly done in the beginning stage of theory development, except when, as Suppes (1960: 296) points out, the notion of model is used in arguments against the general plausibility of a theory. In these cases the theory is (conceptually) extended to a now domain (where the scientists expect the results in the new domain to be different from those predicted by the theory) by constructing a model of the theory in that domain. I agree with Suppes that this aspect of the use of models need not however be restricted to Gedanken expeniments."

Giere (1991: 23 - 27) also discusses the various kinds of model that scientists use 
through the course of the scientific process. He identifies four different kinds of model, but I shall here discuss only the two more heuristic ones:

- Scale models are physical models built to scale and are rarely used, although they may play important roles in the formulation of theories, for example the model of DNA, built by Watson and Crick.

- Analog models are most useful in the beginning stages of scientific research, since they are usually discarded after theory formulation. He (Giere, 1991: 23, 24) cites the familiar example of the solar system as an analog model for an atom. In this model the electrons and the nucleus of an atom are said to be analogous to the planets circling the sun. (The idea being that if an atom could be magnified by whatever measure required, one would have an observable object with a structure similar to that of the solar system correspondingly reduced.) This model was very fruitful to scientists working in the first half of the century, especially in the ways in which it showed atoms not to be analogous to the solar system, the general point being that "a good analogy leads to its own demise" (Giere, 1991: 24).

\subsubsection{Variations on non-statement uses of the notion of "model"}

Wojcicki (1979: 158) sums up the different usages of the notion of model that philosophers of science should keep in mind as follows: "... while the semantic concept of a model is in common use among logicians and mathematicians, empirical scientists almost unanimously opt for the 'mathematical' meanings of 'model' in its syntactic (a set of equations) or semantic (a mathematical entity which is to represent an intuitive concept) sense" (ibid.).

The followers of the "semantic" approach (Suppe, Van Fraassen, Giere, Wójcicki, Przelewski, and the various structuralists) also have among them a few variations on the use of the notion of model. In general all of these support the idea that a scientific theory has to be logically reconstructed in terms of a description of its set of models, which is taken as the structures in which the theory's domain can be modelled. They also view models in the 
Tarskian sense in so far as these models are taken to be relational structures "for which all the sentences of a theory express true properties about the structure when the latter acts as an interpretation of the theory" (Da Costa \& French, 1990: 250). I shall here only comment on Van Fraassen and Giere's uses of the notion of "model", since the intricacies conceming the structuralist interpretation of the notion are best discussed as part of their programme which shall be done in Section 3.3 - and Wojcicki's notion of theoretical models - see Section 3.6 - differs only marginally from what follows.

Van Fraassen (1980: 43) states clearly that any structure that satisfies the axioms of a theory by making the axioms true is called a model of the theory. He (Van Fraassen, 1980: 44) points out that scientists usually use "model" as referring to "type of structure" rather than a specific structure, in the sense that some parameters are left unspecified in their description of the structure - he gives the example of the Bohr model of the atom which was intended to fit hydrogern atoms, helium atoms, and so on. Van Fraassen's own use of the term is directed by his notion of empirical adequacy and thus focuses on the identification of empirical substructures of models of theories, since establishing the empirical adequacy of a theory depends on being able to show that the structures which are described in experimental and measurement reports conceming the theory ${ }^{20}$ are isomorphic to the empirical substructures of a model of that theory. A more in-depth discussion of Van Fraassen's semantic approach follows in Section 3.4, but I want to point out briefly that my notion of empirical models - see Chapter 2 - is very close to his notion of empinical substructures, although $\mathrm{t}$ completely differ from his attitude towards the role of theoretical terms in all of this. More about this in the following chapters.

I have referred in the previous section to Giere's identification of the various uses of the notion of "model". The semantic notion of "model" that he discusses is in terms of what he refers to as "theoretical models". According to him (Giere, 1991: 24), this interpretation of the notion of "model" is the kind most often used in science. Giere (ibid.) uses the analog of maps to set out the characteristic features of theoretical models. A map is not the same thing as the thing it represents, although some kind of relationship does exist between a map and 
the object "mapped". This relationship is according to Giere $(1991: 25,26)$ a relationship of similarity. A theoretical model (map) exhibits a certain similarity of structure with the thing mapped. Although theoretical models are incomplete in the sense that they exhibit only selected features of their subject (as maps do), still they are "... similar in some specifiable respects and to some specifiable degree of accuracy" (Giere, 1991: 26). I shall comment fully on the notion of 'Uheorelical model' in my discussion of Giere's semantic approach in Section 3.5, and thus suffice it to remark here that although Glere seems to see some kind of semantic or perhaps interpretative link between theoretical models and certain aspects of reality, he still claims these models to be "false", and thus creates a few uncertainties concerning his notion of "similarity of structure" referred to above.

Apart from the distinction between models used as analogies and mathematical models, what often happens in the applied sciences is that the notions of "model of a theory" and "theory of the model" are nol always distinguishable. Suppes (1960: 289) points to an example of such confusion often found in the behavioural sciences and mathematical statistics. Here the word "model" is usually used to mean the set of quantitative assumptions of the theory, which in logical terms are synonymous with the axioms of the theory. The model is here a linguistic entity in contrast to the Tarskian use according to which a model is a non-linguistic entity in which a theory is satisfied.

Suppes (1960: 291) claims though that the set-theoretical use of the notion of "model" is more primary than the empirical scientific use of it. In my approach the so-called "physical model" used in the empincal sciences ${ }^{21}$ may perhaps play a role at the lowest most concrete level of the construction of what I refer to as the "conceptual intended model" in the beginning stages of theory formulation (see Chapter 2). However, the Tarskian notion of "model" equivalent to Suppes's "set-theoretical" notion - is much more important and is applicable both to the role I assign to models in the initial stages of theory formulation and the role I assign them in the interpretative or application stage of the theory's development. ${ }^{22}$ 
1.2.4 The implications for philosophy of science of the use of the notion of "model" in econometrics

As an example of the use of the notion of "model" outside the natural sciences, I briefly look at certain aspects of the meaning and use of this notion in econometrics. This illustrates that my model of science is not necessarily limited to the natural sciences. I shall refer to the economic sciences again in Section 2.4 when I discuss the meaning of the notion of an "empinical model". Nancy Cartwright $(1989,1995 \mathrm{c})$ often employs the econometric use of the notion of "model" to make this point too, and also to exemplify certain features of the relations between theories, their models, and reality.

In econometrics a "model" is generally a class of models in the logical sense. Note however that as early as 1968, John Harrod (in Wolfe, 1968: 180) points out the difference he sees between physics and economics and the various uses of "model" in the two sciences as resting on the fact that in physics, because there is no direct access to reality, "one can never compare the model with the reality", while in the case of economics, "all the entities with which economics deal are, in principle at least, directly observable" (Harrod in Wolfe, 1968: 183). This I find difficult to agree with. What does an actual instance of a "free market" look like then $?^{23}$ It seems far more probable if modern economics and econometrics are taken into account, that economists are in general likely to confuse the notions of "theory" and "model" such that their theories are mathematical models in the Tarskian sense, and their models are the linguistic expressions that the supporters of the statement approach call theories. ${ }^{24}$

Be that as it may, Nancy Cartwright's various references (especially in Nature's capacities and their measurement, Cartwright, 1989) to the similarities between the interpretation of equations or sets of equations in econometrics and the interpretation of scientific theories offer perhaps the best example of the versatility of the notion of "model". Understanding the intuitions behind these econometric calculations also goes a long way to illustrate the problems conceming the idealised nature of models that will be discussed in what follows. Therefore I shall briefly here set out an example she discusses in this text 
(Cartwright, 1989).

Cartwright (1989: 149) uses the methodology of econometrics and the nature of its equations and the relations between them to illustrate the kind of stability she associates with the capacities of nature. I shall discuss her notions about the so-called "capacities of nature" in Chapters 4 and 5, but for now, this example is simply meant to give the reader - by analogy to the natural sciences - an idea of the underlying intuitions about the relations between theories, their models, and the aspects of reality in question. (Remember that where the term "model" is used in what follows, mostly we would use "theory", and vice versa.)

Cartwright's example: (Cartwright, 1989: 149ff.): Consider the following equation (which represents a price-demand curve):

$$
q=\alpha p+u \quad \ldots \text { Equation }(D)
$$

where $q$ represents the quantity demanded, $p$ the price, $\alpha$ the strength of the tendency of the price to influence demand, and $u$ the factor changing the character of the equation from a deterministic one into a probabilistic one. Note that this equation plays the role of a fundamental law in Cartwright's terms, which is an axiom (or the set of axioms) of a "scientific theory" in my terms.

Now, keep in mind that econometrics fall in a tradition of economics in which it is accepted and assumed that economic theory studies the relations between causes and their effects, and that, therefore, this equation (Equation $(D)$ ) can be taken to represent a causal relationship. ( $\mathrm{am}$ not implying here that all explanations in the natural sciences are causal, but again, the kind of relations at issue here may be seen as analogous to and illustrative of certain relations in the natural sciences.)

Economic data and economic theory (models) have two characteristics (Cartwright, 1989: 149,150$)$ that are particularly interesting if the analogy between econometric methodology and the methodology of the natural sciences is taken into account:

- an econometric theory (model) is a system of simultaneous equations, and - $\quad$ some - or sometimes, all — of these equations include "random" terms which reflect the influences of numerous erratic causes in addition to the few "systematic" ones. 
What is important to Cartwright's - and our - aims here (Cartwright, 1989: 150), is that, given all of the above, Equation $(D)$ assumes a stable tendency of price to influence demand and that the strength of that tendency is fixed and measurable, denoted by $\alpha$ in Equation (D). The point of any econometric model (theory in our terms) is that its equations represent clearly the fact that something remains constant under change (the $\alpha$ of Equation $(D)$ ). Cartwright (1989: 152) quotes $O$. Duncan as follows: "... if we did not have hope that at least some features of our model [theory] would be invariant with respect to some changes in circumstances ... [i]f all the model [theory] is good for is to describe a particular set of data ... then we might as well forego the effort ...".

Consider now (Cartwright, 1989: 152) what happens if the amount of television advertising is added to Equation $(D)$ as an independent cause that may also influence the quantity demanded. Then we get

$$
q=\alpha p+\beta r+u \quad \ldots \text { Equation }(D)
$$

We add $r$, but assume $\alpha$ stays unaltered. Thus, with or without $r$, we assume the price elasticity to remain unchanged. Cartwright: $(1989: 152,153)$ writes: "It is important to see how peculiar this is. From the point of view of the new equation the old equation expresses a relationship that holds in one particular circumstance: $r=0$. When $r$ is $0, p$ and $q$ have a familiar distribution: a bi-variate normal. In principle, the distribution of $p$ and $q$ could be entirely different when $r$ takes on some different value. But the way the equation is written denies that. The method of writing the equation assumes that, however $p$ affects $q$, that is something that stays the same as the situation with respect to $r$ changes, however it does so". Thus, what happens in econometric methodology is that parameters ( $p$ in Equation $(D)$ ) are established in one context that are assumed to obtain in entirely different situations.

In econometrics, Cartwright (1991b: 8) explains,

[t]he fundamental equations of the model [theory] are supposed to describe separate mechanisms that operate independently from each other. Hence, it should be possible to manipulate the background structure so as to bring about a change in one of these equations without affecting the others - that 
is, to change one mechanism at a time while leaving [the] others intact. These equations are then said to be autonomous with respect to each other. The derived equation, which describes the actual behaviour that the system undergoes, is not autonomous with respect to these others: change any of them and it will change too. It is called a confluent equation. The resulting relationship between the basic explanatory equations of the model [theory] and the descriptive confluent equations appears to parallel that in physics between fundamental laws and their phenomenological consequences.

This relationship is in model-theoretic terms analogous to the relationship in science between theories and their models.

Let us now turn to the issue at hand - namely to work out a model-theoretic realist interpretation of science. 


\section{CHAPTER TWO: A MODEL-THEORETIC ACCOUNT OF SCIENCE ${ }^{25}$}

\subsection{Introduction}

What is it that philosophers can or should say about science? Should we explain the actions of scientists? Should we explain the methodology of science? Should we explain the process and progress of science? Should we study the knowledge claims offered by scientists and decide which of these are so-called "scientific" knowledge claims? Should we study the history of science and its influence on current scientific practice? What - if anything - should we say about reality and the links - again, if any - that exist between science and reality?

In his article on philosophy of science in The Oxford Companion to Philosophy (1995) David Papineau describes philosophy of science as being divided into what he calls the "epistemology of science" and the "metaphysics of science". He claims the former to be concemed with questions about the justification and objective nature of scientific knowledge and the latter to be occupied with "philosophically puzzling aspects of the reality uncovered by science" (ibid.:809). I agree that philosophy of science consists of, among other things, an epistemology of science. I would also agree to describe such an epistemology in terms of justification for scientific knowledge, but then only if it is understood to imply that a scientific epistemology should also - perhaps before anything eise - offer an analysis of the nature of scientific knowledge, and the process by which we come to such knowledge claims. As far as a metaphysics of science is concerned though, I have a few problems with Papineau's characterisation. Much rather than any kind of metaphysical musings conceming philosophical questions about the "philosophically puzzling" parts of reality that science "uncovers", a philosophy of science should offer an ontology of science. If we do assume as Papineau obviously does - that science can "uncover" certain aspects of reality a philosophy of science should surely focus rather on the structure of such an enterprise than that of reality? We are way past the time of so-called "natural philosophy", and should acknowledge that now - as has been the case for many centuries actually - philosophy of science is about science and not about reality. Of course, if it is assumed that science 
"uncovers" aspects of reality, we have to say something about reality as it is linked to science, but never should we, as philosophers of science, again try to write an ontology or (ontologies) of reality.

But, what then of realism? I shall argue in this thesis that the only kind of realism possible is indeed one that focuses more on science than it does on reality. For a workable scientific realism the only necessary and sulficient condition that is concerned with the nature of reality is, simply, the assumption that there exists such a reality independent of whatever happens in science. All the other conditions for such a realism should be concemed with science - its nature, its progress, and its justification. For, how can we claim that science is about reality if we do not study science and its processes? Perhaps it seems as if this question implies an equally strong motive for studying reality. However the assumption of the independent existence of reality takes care of that half of the question, simply because, in the end, a philosophy of science is about science, and science is about reality. When it comes to studying the links between science and reality, the original existential assumption conceming reality may be augmented by certain general ("common-sensical") ${ }^{26}$ ontological onentations conceming the nature of reality; for instance its complex and nich nature, as well as its similarities and regularities, should be taken into account. But the bottom line remains the same since, even then, the reason for these ontological orientations is that the way science links itself to reality cannot be motivated or understood without these orientations. The undertying reason for the complexity of the science-reality connection is that science and reality represent different logical classes and therefore they cannat simply be compared to each other directly - especially not in a one-to-one relation of correspondence - without committing a chain of serious category mistakes. See Chapter $\mathbf{4}$ for my discussion of Cartwright's views in terms of this issue. In Chapter 5 I shall come back to my own views on realism in the light of the model of science developed in this chapter. A discussion of the process of science in model-theoretic terms, analysing the development of scientific theories from their origin to their applications, forms the foundation of a model-theoretic realism. One cannot have the one without the other, therefore I shall begin by analysing science and its 
enterprises model-theoretically (in the remainder of this chapter), then I shall move on to discuss some of the main non-statement analyses of science (Chapter 3), then I shall comment on and discuss Cartwright's analysis of science and her specific kind of realist oriented problems (Chapter 4), and then, I shall discuss the realist features of science in model-theoretic terms (Chapter 5).

I am advocating a philosophical approach to science which introduces a mediating factor (the role of "models") between scientific statements and real objects. In Chapter 5 I shall discuss the realist implications of such an account of science. I shall also show that such an approach offers the means via which the implications of the fact that there is no unique kind of empirical linkage between theories and real systems - but rather a variety of many-to-many links between theories, their (conceptual and empirical) models, and systems in reality - may be satisfactorily examined and made sense of - still within a realist context. A model-theoretic account of science implies an articulation, a kind of "doubling - by splitting - up" of the relations between scientific statements and objects in reality, because it implies these relations have to be regarded as "filtering" through the models in the case of both the formulation of a scientific theory and its application. In this view a conciliation of the statement and non-statement view of science is achieved.

The model-theoretic analysis of the process of science that I propose is done in terms of a model of science that offers a rational (conceptual) reconstruction of the "life" of a typical scientific enterprise, and is offered against a stratified view of the process of science. This stratification is three-fold: it consists of an empirical level, a middle conceptual level, and a linguistic level. ${ }^{27}$ The terms of the first level are very particular, those of the second are more general, although still specific, while the terms at the final level are at the highest level of generality. The relationships between the levels are complex (and often interchangeable), but will be presented as a development in time from the first level through the middle level to the third level, and then back again.

The "purely empirical" level I interpret as based on the existence of vanious systems in reality and consists of our interactions with them, while I view the final level as a level of 
linguistic systems at which a scientific theory is formally formulated and suitably expressed in some appropriate language. The middle conceptual level is a very complex one in the sense that it has various facets that, in their turn, may be seen in terms of a certain kind of hierarchy. It is at this level that models both interpreting scientific theories and making them true are constructed, and, also at this level, the issue of adequate reference to real systems is examined. I claim the latter relations of adequacy to consist of various scientific experimental and observational activities, which may lead to the establishment of a relation of isomorphism between (a substructure of) some model of a theory and some "empirical" model of some real system and the expenimental relations executed in that system.

In this chapter, I shall concentrate on the nature of scientific theories, their relations to models and to (systems in) reality, and the implications thereof for the nature of scientific knowledge. The issue of realism will be addressed separately in Chapter 5.1 shall start off with a discussion of the formulation process of scientific theories, i.e. their "coming-intobeing". Then, in Section 2.4 I shall discuss the matter of applying and interpreting scientific theories. Finally, in Section $2.5,1$ shall conclude this chapter by making a few remarks on the nature of scientific progress as it is depicted by a model-theoretic approach to scientific theories. The issues discussed in the last two sections of this chapter shall be picked up again and worked out finally in terms of their realist implications in Chapter 5.

\subsection{Terminological note}

In this chapter, and also throughout the rest of this thesis, I shall take examples from the natural sciences - mostly from physics and astronomy - to support and illustrate my arguments. As mentioned in Chapter 1 a model of science such as the one that I shall set out in this chapter works very well for the natural sciences, because all three main aspects of such a model are simple and clearly portrayed in the natural sciences. As far as the aspects of reality studied go, an electron is a far simpler concept than a human being; the models employed at the "middle" level of my interpretation of science can often be mathematical in the natural sciences, which they cannot necessarily be in other sciences; and finally, the 
languages can be formalised quite easily in the natural sciences (in first-order logic for instance), while other sciences often use full natural language which is tremendously more complex. I am convinced though that the model that I am proposing for the philosophical interpretation of science is also applicable to the so-called "social sciences", although the slage of theory formulation and the interpretative stage of the scientific process will differ from that of the natural sciences as far as certain emphases on context dependency and other related issues are concemed. ${ }^{29}$ Apart form referring to certain parallel issues in economics and econometrics here and there, the scope of this thesis however does not allow me to go into these matters in any more detail.

Before proceeding, it is necessary to give at least an informal explanation of the notions of "theory" and "model" as | shall be using them. Let us choose a first-order predicate language, $L$, in which a deductive theory $T$ is formulated. ${ }^{29}$ The only condition I set with regards to language $L$, is that it should be appropriate for formulating statements about mathematical structures. Let us say that theory $T$ is the (deductively closed) set of all formulae which can be deduced from a consistent set (system) of axioms, $\Sigma_{1}$ in formal language $L$. Now, in this language, $L$, there will be - among other things ${ }^{30}-$ an infinite, countable set of individual vanables and a nonempty set of predicate letters. Then, a mathematician (or "scientist" for my purposes) may give meaning to these symbols used to formulate the sentences in theory $T$ in language $L$, by constructing a certain mathematical structure, call it $U$, suitable to be described by the language $L .{ }^{31}$ An interpretation of language $L$ will consist of a set over which we consider the individual variables to range, and predicates or relations defined on this set as interpretations of the predicate symbols in L. Thus as soon as every n-ary predicate symbol in language $L$ is associated with an $n$-ary relation in structure $U$, we can say that this mathematical structure is an interpretation of the language $L$, and thus, by implication, of any sentence in $L^{32}$ Note that of course, for every other definition of the domain of the mathernatical structure and of the relations defined on it, one is confronted with another interprotation of the language. There are thus no "rigid designators" across interpretations. A model of any formula such that every free occurrence of variables in it 
refers to an element in the domain of an interpretation of $L$ by means of a specific valuation, will be an interpretation under which that formula is true by the specific valuation defined for its (the formula's) variables. ${ }^{33}$ Now, a sentence is a formula with no free occurrence of variables. Thus the definition of the truth of a formula implies that a sentence will either be true under an interpretation by all possible valuations or false under all valuations. Hence for sentences we may speak of truth under an interpretation without mentioning valuations. And, a set of sentences is true under an interpretation if every sentence of that set is true under that interpretation. Thus a model of a theory (being a set of sentences in some formal language $L$ ) will be an interpretation under which that set of sentences (i.e. the theory) is true. ${ }^{34}$

\subsection{The formulation of scientific theories}

Now, tuming to the epistemic process leading to the formulation of a scientific theory, the following. No-one - not even scientists - ever studies reality in all its complexity. The way in which we come to knowledge is determined by acts of abstraction and simplification. Thus, rather than focussing on the colourful richness of reality, scientists typically will decide to focus on a particular aspect of reality. Moreover, intensifying their initial selective actions, scientists will also decide to concentrate only on particular features of the real system they have picked out.

At the start of a particular line of research, the first encounters between scientists and the relevant system in reality have an interesting feature. Although traditionally viewed as happening at the lowest level of scientific activity - "lowest" in the sense of least abstract and not least dependent on historical, social, and cultural factors - these encounters are already not "objective" in the sense of being neutral to any kind of extemal influence. This is because of the influence various contingent factors have on the actions of scientists and their arguments. These factors range from extremely specific to broad combinations of general factors influencing scientists at a given time.

They include personal factors such as the personal interests of scientists involved, their 
particular research goals, and the social context in which their research is done. Then there are also factors that pertain more to the "theory-ladenness" of the choice of experiments, the interpretations of data, and so on. These factors include the paradigm or research tradition in which the scientists are working, the state of their discipline at the time, the level of technology and experimental apparatus available at the time to those particular scientists, and also the body of "already established" theories (as the background) against which these particular scientists will work. Also included here are factors to which Gerald Holton (1995) refers as "themata". These factors are on the one hand, a cross between the specific motivation behind the choice of addressing results and problems within one particular scientific framework rather than another, and, on the other hand, the scientists' world view at the time. ${ }^{35}$ In this sense, I agree with the constructivists: no scientific activity takes place in some kind of objective vacuum.

The inherent conditioning, or refining, abstractive nature (and potentially idealising power) of activities carried out at this level leads ensuing activities to a level more general in scope than that of the original encounters with specific aspects of reality. At this level scientists create conceptual "models" - which I call "intended models" for obvious reasons - of the real system in question. These models are obviously not (yet) formally identifiable as interpretations of any sentences of the language in which the final theory will be formulated. However, after theory-formulation, at the stage where possible interpretations and (empirical) applications of the theory in question are considered, it will become clear that the intended "model" of the theory in question is also one of the (possible) mathematical models (i.e. interpretations under which the theory in question is true) of the relevant theory. (Thus, at the formulation stage of scientific theories, my use of the term "model" is perhaps not strictly in the same sense as the one model theorists use.)

For example, let us consider briefly the formulation of Newton's laws of motion and his law of gravitation. Newton wanted i.a. to continue Kepler's research about the movement and positioning - of the planets in our solar system. Kepler's laws onginated, it seems, largely because of his own interest in specifically the movement and positions of the planets 
in our solar system. His intended model thus may be said to have been supported by data concerning only these (and related) planetary features. His research was based on observational data regarding the positioning of the planets at different times, much of which was the original work of Tycho Brahe. His interpretation of his observational data would have been, for instance - and probably among other things - influenced or "laden" by his mathematical idealism - e.g. his claim that the planetary obits should fit exactly into nested Platonic solids. Finally, Kepler's research culminated in his formulation of his three laws the first two in 1609 and the third in 1618.

Now Newton could not study all the complexities of our solar system as it manifests itself in the manifold of reality. He was also, as mentioned above, interested in examining planetary motion in our solar system. Thus he identified the details necessary for his research goal by abstracting from this system in reality only those specific features in which he was interested. He would have discarded, for instance, the fact that the sun's rays are hot, that Mars seems to be reddish in colour, and so on. But, in this way, because these abstractions were so closely guided by his intentions - and certainly influenced by both Brahe and Kepler, and also Galileo's findings - he would never really have been dealing with the bare data that he had extracted from reality. He would, rather, in fact, have been dealing with a conceptualised model of our solar system that would in the end lead him to the formalisation of the theory of solar systems itself.

In order to study the dependence of the force of gravity on the distance from the centre of the earth, Newion compared the fall of a stone (the alleged apple) on the surface of the earth with the motion of the moon. Newton discovered that the "... forces of terrestrial gravity decrease as the inverse square of the distance from the centre of the earth" (Gamov, 1962: 62). He consequently generalised this result to "all material bodies in the universe" (ibid.) and so formulated his universal law of gravitation.

Scientists thus conceptualise their objectives in the light of the data they gather - and may still be gathering - with an eye on their research goals and guided by the specific scientific tradition, community, theoretical network or paradigm they are working from. ${ }^{36}$ The 
creative context of this stage of the scientific process offers scientists the chance to test and constantly reformulate their conceptual structures and to receive results under the conditions set by their goals and the context within which they work. This implies that these (intended) models have an idealised nature ${ }^{37}$ in the sense that they are the results of extremely focussed actions which typically disregard factors in the empirical system in question that could muddy the waters of their research.

The progression of generalisation common to this stage of science may perhaps roughly be logically reconstructed to range from

- scientists' initial sensations (possibly mediated by an apparatus) of "real" objects and their behaviour in some real system (broadly, of aspects of reality), to

- the construction of percepts of these sensations, to

- the construction of concepts of these percepts, to

- the construction of conceptual models which are structured sets of these concepts, and which

- may then - in certain sciences - culminate in the formulation of mathematical models.

Finally, these (abstracting) actions culminate ${ }^{38}$ in the formulation of a general (abstract) theory - expressed in some suitable language - in the field of research in which the relevant scientists have been working. The nature of this level at which theories are formalised is abstract, general, and simple in the sense that the values (meanings) of the parameters in the general theories are essentially unconditioned and the meaning of theoretical terms (such as "electron" or "mass") is in principle open to valuations or interpretations made by scientists interested in applying or implementing the theory. This implies naturally that a potentially infinite number of conceptual (or mathematical) models can be constructed of one and the same theory.

The aim of Kepler's research surely was to formulate some kind of law (or laws) concerning planetary motion. It is sometimes claimed that Kepler's laws do not constitute a scientific theory, however. For instance Diworth (1994b: 135) claims:

The main reason usually given for Kepler's laws, taken together, not ranking as a 
theory is their unequivocally empirical character - i.e. the fact that they are 'instantiated' in the sense that they refer to the individual planets in the solar system and, unlike a theory, are capable of being tested more or less directly. The present view [Dilworth's] supports a distinction along these lines, and in fact provides an explanation of it, viz., that, unlike Newton's theory, Kepler's laws are not integrally related to a model ....

Well, it is indeed the case that Kepler's laws are very "empinical" and less general in scope than Newton's laws of motion and his law of gravity, since the latter do not give any particular value or parameter to their theoretical terms. ${ }^{39}$

However, Kepler does generalise from his intended model in the sense that his laws are about the motion and position of all the planets - or any planet - (known to him) in our solar system, and not particularly about any one specific planet. Moreover, the application of his laws to the motion and position of any one planet may be seen to imply the construction of a specific conceptual model concentrating only on the particulars concerning that specific planet. Although his laws are indeed far less general than Newlon's, and also far more like the kind of "empirical" theory philosophers in the Popperian tradition advocate, his laws can be viewed as part of an "intellectual system" of the kind Torretti (1990: 24) supports. Torretti's argument comes down to the following: Should it be found (as perhaps from a certain perspective it was discovered much later) that a particular planet does not obey Kepler's laws it would imply a revision of our scientific thoughts concerning planetary motion. I also take this as sufficient motivation to view Kepler's laws as comprising a theory (albeit perhaps a "lowlevel" one).

It is the case that Newton's laws of motion and his law of gravity could in fact finally explain Kepler's laws, and thus that Kepler's laws may perhaps not be said to explain the positioning of the planets but rather merely to describe their motion. ${ }^{40}$ I think that we have at least to accept the legacy of the advocates of the deductive-nomological model of explanation in so far as we accept that scientific explanation is really some kind of inference, the conclusion of which describes the facts to be explained. Generally, Newton's laws explain 
Kepler's in the sense that a conceptual model of Newton's laws may be given by our solar system, and the data giving positions and motions of a particular planet in that solar system may be viewed to constitute an empirical model that is isomorphically embedded in the above conceptual model. More formally, the application of Newton's universal law of gravity to the motion of our solar system's planets around the sun (conceptual model) enables one to mathematically derive Kepler's three laws (empirical model). More specifically for instance, Newton's law of gravitation explains Kepler's third law in so far as it shows that Kepler's third law is based on a force that is exerted towards the centre of the sun and that is inversely proportional to the square of the distance between the sun and the planet in question. However, it has to be noted that although Newton's laws explain Kepler's they do not, after all, explain e.g. gravity - they merely describe it. (I shall briefly discuss the explanationdescription debate again in Cartwright's terms in Chapter 4. This, though, is a very complex debate that cannot be fully analysed within the scope of this thesis.)

Do Newton's laws constitute a theory? Should his laws be viewed as the axioms of this theory or as empinical laws in the Popperian way? In the model-theoretic approach to science that I advocate, the axioms of a theory describe the conceptual model(s) in which they (the laws they represent) are true. Such a model then sets out the calculation of values of certain functions and the interpretation of certain theoretical terms in the context of the model. In this case, for instance, it could mean that bodies are conceived of as "mass-points" without extension. The theory in general sets down the nature of the relations between the terms in its conceptual models. Thus on my account, Newton's laws of motion and his law of gravity do constitute a theory since they are general enough, or broad enough in scope to offer different conceptual models and interpretations of its terms in different contexts of application. ${ }^{41}$ They can be viewed as "empirical" laws only via the interpreting mediation of conceptual models and some empirical substructures representing observational data and other empirical calculations, which brings us to the next section on the interpretation of scientific theories. 


\subsection{The interpretation of scientific theories}

Now, in order for scientists to give "reference" to the multi-interpretable theoretical terms and parameters ${ }^{42}$ of scientific theories, more models - other than the original one leading to the formulation of the theory - may be constructed. In other words, because of the possibility of all these different models of theory $T$ (in language $L$ ), the theory - say in our example, Newton's laws - may be (in principle at least) related to any (mathematical and thus conceptual) model in which it is true and not only to the intended one. If we take the set of axioms, $\Sigma$, to be Newton's three laws of motion and his law of gravitation, expressed formally, then they will hold in any planetary system (because of the general nature in which they were formulated) and then we can pick any "planet" in such a system and be sure that its orbit will be an ellipse and the system's "sun" will be in one focus. E.g. a model in which, say, Jupiter is the "sun" and its satellites are the "planets" may now be constructed.

These models are thus interpretations of the theory ${ }^{43}$, each of which is also in its turn determined by - among other factors - the research intentions and thematic preferences of the scientists wishing to apply or study the theory. Note here that the first most obvious model of the theory is its oniginal "intended" one. However, since different groups of scientists will be applying the theory in question - perhaps for different reasons - at various times, this is not necessarily the model that will be chosen as the one via which the theory is to be applied or interpreted. The intended model is thus in nature no different from the mathematical structures that will be constructed to interpret the theory in such a way that the theory will be true in them. These models simply differ as far as the nature of their origins is concemed, and features common to both - such as the role of thematic preferences - are simply emphasised differently in each case. In this sense for example, the intended models have more of an organising and guiding role in the sense of being the first conceptual means via which scientists are able to make the first abstractions from reality. The conceptual models will give reference and meaning to or "fill in" the content of (some of) the general terms (e.g. electron, mass, velocity, temperature) used in the theory ${ }^{44}$ and specify values for the parameters of formulae in the theory in such a way that the theory turns out to be true in 
these models. The models thus constructed are then obviously mathematical models in the Tarskian sense. ${ }^{45}$

Although more specific than the theory, these models are still general in nature in so far as they are idealisations in the same way as the intended models are. Nancy Cartwright (for instance Cartwright, $1983,1986,1989)$ sees this as problematic as far as the possibility of theories offering descriptions of reality is concemed. I disagree, as will be discussed in Chapter 4. It is impossible to give clear-cut rules or conditions for the adequacy of our conceptions or the real existence of objects in systems in reality, because of the open-ended generality of theories in the sense of the different models in which they may be true and which is a consequence of the "abstract" character of theories and also because of the openendedness of the conceptual models in the sense of their "ideal" nature. The role played by the empirical activities of science in establishing the last referential link between some system in reality and some model of the theory needs a suspension at the conceptual level of the ceteris paribus clauses at play at the linguistic level of science in order to fill in the details that, at this level, have been "idealised" and "abstracted" away. More about this in what follows, and also in Chapters 4 and 5.

Here is an example to illustrate the construction of different models interpreting the same theory. Newton's laws made it possible to calculate very precisely the motion of the planets in our solar system (any solar system for that matter) under the influence of mutual gravitational attraction. Up to 1820, scientists interpreting (or applying) Newton's laws of motion and his law of gravitation to our solar system had worked in a model of these laws (comprising Newton's "theory") which consisted of only seven planets. Then, in 1820, calculations carried out within this model started to give "wrong" predictions, and it became apparent that the motion of Uranus "did not conform to Newton's grand scheme" (Schwinger, 1986: 195). The possibility that the motion of Uranus could be affected by the gravitational attraction of another planet seemed a good solution to the problem though. So, scientists thought of postulating the existence of an eighth planet, and consequently constructed a different model of Newton's theory, now with eight planets. In 1845 John Adams calculated 
the position of this "new" planet - Neptune - in our solar system, and shortly afterwards Urbain Leverrier's calculations confirmed Adams's findings.

Applications of the same Newtonian "theory" (his three laws of motion plus his law of gravity) includes the "discovery" of Pluto in 1930 as the result of theoretical calculations based on the universal law of gravity. Also Newton gave the first explanation of the "precession of the equinoxes" since the time of the Greeks by applying his law of gravity to the motion of the earth. And, a last example, aspects of the motion of the tides of the sea could be explained by applying the universal law of gravity to the earth's perihelion and aphelion motions (i.e. the movement of the earth far from and close to the sun). Thus new information results in different (new) models still constructed to attain the same (previous) goal, but also different aims result in different models.

I take the relations that exist between some theory and the mathematical (conceptual, semantic) models that are interpretations of the theory's language and in which the sentences of the theory are true, as the first set of relations that determine the possibility of reference to some real system. The goal of a formal logician will be to prove that his deduction (theory) is valid, i.e. true in all possible worlds allowed by the axioms, i.e true in all possible models (in the conceptual system), one of which may or may not be in its turn "about" some system in reality. Thus, for the formal logician, the question of whether it is possible to construct a "second set" of interpretations or models (to retrace the steps of the original scientist representing the group of scientists that "formulated" the theory in question - even further back to reality), is rather irrelevant,

Scientists, however, will definitely be interested to know whether one of the conceptual models of their theory can have a system in reality as some further interpretation or model, because they formulated their theory precisely to enable them to make some sort of claim about a certain aspect of some real system. The method of verification of each of these (conceptual, mathematical) models (i.e. how well do each of them reflect the system in the real world?), will be decided by the specific nature of the specific (conceptual, mathematical) model in question, as well as by the nature of the specific real system in question. It could be 
that an observation through a telescope is needed, or an observation through a microscope, or some sort of calculation, which has less to do with observation, and so on and so on. In other words, neither Tarski, nor anyone else, could or can really give a general criterion for the truth of the sentences in this last set of interpretations.

Should some of the elements and relations of one such a conceptual model be interpreted to correspond to objects and relations of some system in the "real world" however, I claim a further mathematical model may be identified such that its objects and relations (representing the relevant empirical data) constitute a mathematical structure which is (isomorphic to) a substructure of the relevant conceptual model. If the phenomena in some real system and the experimental data concerned with those phenomena are logically reconstructed in terms of such a mathematical structure - call it an "empirical" model - the relation of empirical adequacy (characterised by the various actions mentioned above) then becomes a relation which is an isomorphism between the empinical model and some substructure of the relevant (conceptual, semantic) model of the theory in question.

For example, take a conceptual model of the theory constituted by Newton's laws of motion and his law of gravity as the elliptical orbit of some planet - say Pluto - around the sun, with the sun in one of its foci. Say 117 individual observations of Pluto on this elliptical course are made by scientists working in this model. Then these observations, jointly (interpreted as 117 points, at different times, on an ellipse) represent an empirical model of the theory. ${ }^{46}$

Let us take a brief look also at what happens for instance in econometrics as far as empirical models are concemed, before we continue, Mary Morgan (in De Marchi, 1988: 199) points out that econometricians have always been occupied with finding "applied counterparts to theory that "worked" with reference to observed data" (ibid.). She (and econometricians in general) might however have a slightly different interpretation of the notion of "empirical model". For instance she (De Marchi, 1988:200) remarks that the onginal goals of econometricians were to make economic theories more "concrete" and to measure the constant parameters of the laws of these theories, and since the "theoretical models" (which 
can either refer to the theory or a conceptual model of the theory in my terms, since economists seem to use these notions sometimes interchangeably) were not measurable, finding empirical models that could be subjected to measurement became necessary. In my terms empirical models are the means via which the mathematical or statistical data of experiments are linked to the more qualitative content of a conceptual model of a theory. So perhaps the difference between our notions seems for present purposes negligible enough not to spend any more time on it.

However, although examining the econometric case is illuminating with regards to ours, it becomes apparent that Morgan is indeed somehow conflating the notions of conceptual and empirical model set out above. She (De Marchi, 1988: 201) sets out three points in the process of "finding an empirical model to match the theory involved [and] making the theory operational" (ibid.). Briefly they are transforming the "verbal theory" into mathematical form and deciding on values for variables, dealing with the ceteris paribus clauses under which the theory is taken to hold, and, setting down the specific time frame for each theory. At least the first two points seem to be worthy of comment for our purposes. The translation of the "verbal" theory into mathematical form is in my terms done either at the linguistic level if Morgan means here the mathematical formalisation of sentences in natural language, or at the conceptual level, at the time of interpretation, if she means to emphasise the determination of values of variables. In either case this formalisation is not necessarily part of finding a suitable empirical model, although the specification of time frames as part of the specification of parameters, depending on the theory in question, might be.

A more serious difference is related to her point about the ceteris paribus clauses necessary for the theory to hold in the general way that it does. These conditions are already "cashed out" when a conceptual model of the theory is constructed in the sense that the "initial conditions" needed to make the specific calculations characteristic of actions regarding the conceptual model are formulated as part of the said model. The idealised character of conceptual models is not so much a result of the ceteris paribus conditions at play at the theoretical level as simply a result of the fact that these models are still models of a particular 
theory. The empirical models in my terms are then even less related to such clauses. Of course, even when experiments are carried out, it is the case that certain factors are kept stable so as to be able to examine a specific aspect of the real system being studied. These factors are however far more specific than the ceteris paribus clauses and are rather a result of the specialising way in which science is typically practised. The relations between science and reality cannot be anything other than somehow idealised, given the complex nature of reality, although the construction of conceptual models of a theory starts the suspension of the generalisation implied by scientific theories' ceteris paribus clauses. More on this in Chapters 4 and 5.

Thus, my notion of empirical models implies that both the models assigning reference to the general terms of the theory and the empirical models representing the (usually quantifiable) aspects of verifying the conceptual models of some theory have to be "constructed". "Construction" in the first case is more formal and less complex than "construction" in the second case. In the second case it implies that data about some system in reality is "found" or "discovered" via the necessary experiments co-determined and executed in terms set out by the relevant (conceptual) model of the theory. And the results of this "construction" may then be represented by some empirical model in which those results about the relevant real system are true. Here there is a correlation between Morgan's (De Marchi, 1988: 208) remarks concerning the status of empirical models in econometrics and the status of empinical models in the natural sciences. She (ibid.) writes "... the empirical models that econometricians [work] with [are] a sort of halfway house, formed to capture the correspondence between theory and data ...". Well, empirical models in my terms also have to "bridge" the gap between the conceptual models of a theory and the quantified data concerning some system in reality. A last remark concerning Morgan's article: she (De Marchi, 1988: 207) remarks that Haalvelmo (1944) stated that “... in the absence of an experimental framework in economics, econometrics must act on both the theory and the data, making adjustments on both sides in order to get satisfactory models". In the natural sciences this is also true - even despite the presence of an experimental framework (or 
perhaps because of it?). The hierarchy of "tools" needed to bring this about is perhaps just a little more complex.

Note thus that the referential relations between the theory and its models are much more simple - although also neither nigid nor absolutely fixable - than the relations between conceptual models and empirical models of systems in reality. The latter are extremely complex and never passive (or absolute), because in this case there are so many more variable factors to lake into account when considering this more informal and supple relation. I quote Sir Allan Cook's (1994: 141) explanation of the link between observation, models and theory in physics to show this more clearly:

Observation is never an isolated activity. The way that we observe depends on human capabilities and properties of nature. Observation may affect the objects observed and our observational procedures depend upon the state of technology and are guided by theory. The results of observation [represented by my empirical models] have to be derived by procedures that depend upon some theoretical model [one of my conceptual models] as well as upon experimental techniques, ... The harder we question nature, [and] the more fundamental the observations we make, the more dependent are the results on technique and theory.

Moreover we as philosophers cannot tell - especially not "before the fact" - which specific conceptual construct (which interpretation of some theory) provides the most adequate description of some relevant system in reality. ${ }^{47}$ Only science itself can offer us - at some more mature stage of scientific development - an ontology (or ontologies) which can specify the contents or detail of the structures reality contains and the particular ways in which they behave. ${ }^{48}$ Thus, neither the adequacy ("truth") of our conceptions nor the "reality" of the system as described by some theory, is absolute, because both are products of epistemically relative interpretations and subject to change. "Adequate" scientific statements may, indeed, say of reality that it is the way it is. It is, however, only through the mediation of (conceptual and empirical) models that this can be established, and never directly by somehow companing theories to reality. More about this in Chapter 5. 


\subsection{The process of science}

\subsubsection{Introduction}

Thomas Kuhn's The structure of scientific revolutions (1970) represents a watershed in philosophy of science in terms of views concerning the nature of the scientific process, culminating in claims about the nature of science's progress, that cannot be ignored in a realist analysis of science such as the one offered in this thesis. Although Kuhn's writing perhaps never touches directly on the statement/non-statement debate, its use of notions such as "paradigm" and "scientific community" invites comment from any model-theoretic account of science, while Kuhn's notion concerning "incommensurability" invites comment from a realist point of view. In what follows I shall briefly discuss the nature of scientific progress in terms of a model-theoretic realist account of science, to show how Kuhn's revolutionary periods may thus be interpreted and accommodated. I shall also discuss the process of science in terms of the distinctions and common features between the Kuhnian notion of "paradigm" and my notion of "conceptual model". I shall mainly concentrate on the Kuhn of The structure of scientific revolutions, simply because that, in the end, remains the "bible" for Kuhnian philosophy of science.

\subsubsection{The nature of scientific progress}

Is the true nature of science revolutionary? Or is the process of science cumulative? Can there be revolutionary periods as well as cumulative periods in the development of science? Is the notion of paradigm essential to the idea of revolutionary change in science? is the notion of model essential to the notion of cumulative scientific progress?

In The structure of scientific revolutions Kuhn (1970:1) advocates a shift of focus in historical and philosophical studies of science from studying "finished scientific achievements" (ibid.) to concentrating on the "historical record of the research activity itself" (ibid.). He (Kuhn, 1970:2) is anguing against the conception that the content of science is somehow "uniquely exemplified" by observations, scientific theories and laws, and so against a rather naïve interpretation of the notion of "development-by-accumulation" (ibid.). Believers in this view of 
scientific progress have the task of showing that once current views of nature, such as Aristotelian dynamics and phlogistic chemistry, are, as a whole, neither less scientific nor more the result of views peculiar to specific scientists, than later theories are.

One of the most powerful arguments against scientific realism - sometimes referred to as the so-called "argument from scientific revolutions" - centres around the seeming contradiction implied by the following question:

- How is it possible, if scientific realism is true, that the history of science abounds with examples of theories that have had great predictive success, but that were eventually "unmasked" by later science as being "unacceptable" or simply "false"?

There are of course various ways in which the argument from scientific revolutions can be approached. The one that seems to me to be the most plausible relates to discussions in recent philosophy of science by philosophers like Nancy Cartwright, and also the nonstatement advocates such as Patrick Suppes, Bas Van Fraassen, Ronald Giere, and a few others. In this context, I approach the problem of the nature of scientific progress by means of the conceptual system of models of theories that I have explained in the above. I claim that the role of so-called "scientific revolutions" in scientific progress may be interpreted as less intemuptive and more continuous if the suppleness of this stage in the scientific process (i.e. the stage at which models are constructed) is allowed to influence our notions conceming scientific knowledge.

Now, Kuhn wishes to focus rather on the historical acceptance of scientific knowledge in a particular time than on any timeless contributions from an earlier science to the present one. To do this it seems to be important that any study of a specific scientific practice should be conducted from the point of view of the scientists working in that particular community at the time and not from the point of view of modern or current science. In this sense it seems likely that such a study may offer nothing more than explanations for the "internal coherence" of theories and for the reasons why they are deemed to offer the "closest possible fit to nature ${ }^{N}$ at a specific time. Kuhn (1970: 4) stresses in this sense that it is indeed the "incommensurable ways of seeing the world and of practising science in it" (ibid.) that 
differentiate between various "schools of science" and not simply methodological failures or differences. He (1970: 118) claims that: "At the very least, as a result of discovering oxygen, Lavoisier saw nature differently. And in the absence of some recourse to that hypothetical fixed nature that he 'saw differently', the principle of economy will urge us to say that after discovering oxygen he worked in a different worlo".

Kuhn goes on to claim (1970: 7) that "revolutions" do not simply imply the addition of one more "item to a scientist's world", but rather result in important changes in the scientific processional infrastructure. They cause the revision of the evaluative measures concerning experimental procedures that are in place at a given time in a given community, change the familiar ways in which entities were conceived or conceptualised up to that time, and Litimately, result in a shift of the "network of theory" through which the given community "dealt with the world" up to that point in time.

In what follows, I shall address the following questions related to the above:

- Is the content of science wholly, and uniquely - or even mainly - given by current observations and scientific theories and laws? In this context, what is the role of models in the process of science?

- If science is studied in the way advocated by Kuhn in The structure ..., how exactly should the nature and role of "paradigms" be understood? What is the difference - if ary - between the role played by models in the process of science and that played by paradigms?

- What is the meaning of Kuhn's "different worlds"?

In his best anti-realist manner, Kuhn (1970: 7) claims that "[s]cientific fact and theory are not categorically separable, except perhaps within a single tradition of normal scientific practice. That is why the unexpected discovery is not simply factual in its import and why the scientist's world is qualitatively transformed as well as quantitatively enriched by fundamental novelties of either fact or theory".

I disagree with Kuhn about the reason why an unexpected discovery is, as he (ibid.) claims "not simply factual in its import". He offers the inseparability of scientific fact and theory 
as justification for this claim. I would, rather than entwining "real" facts and "scientific" theories from the start, instead speak of the layered nature of the process of science and consider each layer and its active factors in turn, and only then go on to study the connections and relations between layers, and between (some of) these layers and aspects of reality.

In this way, at least a conceptual distinction between science and reality becomes possible, and I see that as a plausible way in which to rescue some form of realism and also to escape the overwhelming (epistemological and ontological) relativism implied by the kind of constructivist approach Kuhn advocates. I agree with Bhaskar who states in A realist fheory of science (1978) that the relationship between science and reality seems problematic only if one either accepts the social character of science, but denies that its object of study is independent of all social activity (the epistemic fallacy), or if one accepts the independence of reality, but denies the social nature of science (ontic fallacy). While Kuhn seems to be guilty of the first kind of fallacy, it is worthwhile to point out that the ontic fallacy (which seems somehow most compatible with the view concerning progress-by-accumulation) is not, however, the only other option open to us.

Acknowledging that reality exists independently of the enterprise of science, need mean neither the denial of the social nature of science nor succumbing to views as narrow as those focussing only on the "natural", "fixed", or "objective" nature of the object of scientific study. None of the above implies that for instance theories explaining and describing light as photons (quantum-mechanical entities that exhibit both characteristics of waves and of particles) are not scientifically "more advanced" or "better" than Huygens's theory of light, or theories based on Fresnel's and Young's theories that claimed light to be transverse wave motion. Neither does it imply that I somehow view Planck's, Einstein's, and Feynman's theories about light to be out of reach of further sciertific activities or criticism. Science progresses and scientific knowledge is cumulative in the following way.

Scientific progress is made at different speeds at the different levels of science. For instance, the model of Newton's theory of our solar system which works with seven planets can still be said to refer to an aspect of reality. The question of the truth of the theory is 
model-specific in the sense that it depends on the satisfaction of truth criteria which may differ from model to model and are satisfied differently in different models. ${ }^{50}$ Thus, in my example, Newton's theory may indeed be true in each independent model thereof, by specific valuations under specific interpretations. But the theory - taken in its general uninterpreted form - could not be said to be true because the model with seven planets referred to reality, and then be said to be false, because of the construction of the new, more encompassing model dealing with eight planels. Nothing can really, model-theoretically, be said about the truth (unqualified) of such an uninterpreted set of sentences.

Theories change very slowly, conceptual models more quickly, and empirical models and the empirical data bases (the accumulation of empirical data via observations and experiments) they depict, the quickest. The general theory of relativity was formulated by Einstein (and Hilbert) in 1915. For more than 80 years now physicists have been constructing literally dozens of different types of models - all models of precisely the same theory - to fit the experimental and observational data about the space-time struclure of the real universe, but also to fit individual or even cultural preferences, such as a passionate belief in, or an equally passionate aversion to, the idea of a Big Bang.

Theory changes usually occur only when the possibility of changing and modifying the models of the theory concerned has been exhausted, which confirms the continuity of scientific knowledge. Think of the rotation of the orbit of Mercury. Einstein's theory of general relativity interprets all gravitational interaction as due to the curvature of space-time. Applying Riemann's mathematical theory of curved spaces (of any number of dimensions) to the physically real curved space-time — a four-dimensional space-time - and correlating by equations the so-called "curvature tensor ${ }^{\mathrm{n} 1}$ of the space-time continuum with the distribution and motion of masses, all of Newton's law of gravity's results may be derived at the first approximation. For example, according to Newton's law of gravity planets move along the elliptical orbits with the sun in one focus that Kepler's empirical laws had already determined. According to Einstein's theory of general relativity all motions should be considered in the four-dimensional world of "events" $\left(x_{1}, x_{2}, i c t\right)^{52}$ which is curved if gravitational fields are 
present. The "world lines" (Gamov, 1962: 205) of any material body in the four-dimensional world (representing the history of the motion of that body) must be geodesics or the "shortest lines", and can be calculated on the basis of the general theory of relativity. Exact calculations showed that the elliptical orbit of a planet around the sun does not stay stationary as Newton predicted. Rather it is "slowly rotating with its major axis turning by a small angle in the course of each revolution" (ibid.). This phenomenon is most noticeable in the case of Mercury, which is closest to the sun and has the most elongated orbit of all the planets. Thus no model of Newton's laws of motion and his law of gravitation could solve the anomaly with regard to Mercury's perihelion motion, and so finally a new theory was formulated, some of the models of which can indeed explain these discrepancies. Gamov $(1962 ; 205,206)$ writes "Einstein calculated that the orbit of Mercury must turn by 43 angular seconds per century, and solved herewith the old riddle of celestial mechanics. It was calculated by mathematical astronomers long before Einstein was born that the major axis of Mercury's orbit must slowly tum around because of the pertubations, i.e., gravitational disturbances, of the other planets of the solar system. But, there was a discrepancy between the calculations and the observations amounting to 43 angular seconds per century which could not possibly be explained lin the models of classical mechanics]".

Now, in this sense, I agree with Kuhn that neither the content of science nor any system in reality should be claimed to be "uniquely exemplified" by scientific theories from the viewpoint of studies of "finished scientific achievements". And, therefore, one has to accept the open-endedness of theories as a permanent feature of the total process of science. The terms of an already established theory can be said to be "about" an ongoing potential of entities in some system of reality to give reference to some objects and relations in some model of that theory. The actualisation of this potential requires human action (in the sense of finding or formulating "satisfying" referenlial relations between systems in reality and some models of the theory).

Moreover, as mentioned before, any successful interpretation of a theory in a model is guided by contingent conditions which are context-specifically constructed. The range for 
content) of these models thus cannot be established a priori. The formal (or a prion) property of scientific progress we as philosophers can say something about is the conceptual process of (re)formulating and applying theories in terms of (many different) models constructed to interpret the theory and having the ability to link systems in reality (via an embedded empirical submodel) to the theory itself. This model-theoretic framework makes possible the verisimilitude enterprise of comparing theories as to their truthlikeness (of which more in Section 5.5). And, in this framework it is still possible to show that the progress of science is indeed accumulative. More on this in Chapter 5.

\subsubsection{Paradigms and models}

In the Postscript to The structure of scientific revolutions (1970), Kuhn admits that at least two main and "very different usages" of the term paradigm are possible. I shall in what follows briefly discuss these two usages - paradigms as "disciplinary matrices" and as "exemplars" - in terms of a model-theoretic account of science. I agree that the Strucfure's notion of paradigm in the sense of the context within which theories are interpreted and applied is a valid and unmistakable part of the conceptual processes of science. As explained in previous sections of this chapter, my usage of the notion of model identifies models as components of these kinds of paradigmatic context.

Kuhn (1970: 175) claims that the two main senses in which he meant the notion of "paradigm" to be interpreted are:

- as standing for the "entire constellation of beliefs, values, techniques, and so on shared by the members of a given community";

- and as denoting "one sort of element in that constellation, the concrete puzzle-solutions which, employed as models or examples, can replace explicit rules as a basis for the solution of the remaining puzzles of normal science".

\subsubsection{Paradigms as constellations of group commitments}

Kuhn (1970: 182) acknowledges that scientists would rather speak of shaning a theory, 
or a set of theories, than of sharing a paradigm or a set of paradigms. This led him to formulate the term "disciplinary matrix" to denote that which is shared by members of a scientific community and that accounts for the success of their communication and their agreement about professional judgements. He (ibid.) explains that "disciplinary" refers to the common possession of members of a specific discipline, and that "matrix" refers to the fact that what is shared is an ordered system which consists of elements of various kinds, each needing further specification. Disciplinary matrices (Kuhn, 1970:184) consist of mainly three components:

- first, symbolic generalisations - either formalised, or expressed in words, like "elements combine in constant proportion by weight";

- second, what he referred to as the "metaphysical" parts of paradigms and which he explained as commitments to beliefs such as "all perceptible phenomena are due to the interaction of qualitatively neutral atoms in the void, or, altematively to matter and force, or to fields" and which he described as beliefs in models; and,

- lastly, values such as predictive power, simplicity, consistency, plausibility - and he stressed that the application of values may be considerably affected by individual factors such as personality and biography.

In my exposition of the conceptual process of science, the need for a disciplinary matnix something which is common to a certain group of scientists - is obvious. Whether in the sense of offering the theoretical background against which scientists gather data from systems in reality, or in the sense of forming research objectives and directing conceptualisations of real systems, or in guiding interpretations of theories in their application or implementation, a context somehow common to all scientists of a certain research group is indeed indicated.

Note though, that Kuhn (1970: 184) sees it as the function of models to provide the relevant community with preferred or permissible analogies and metaphors, and so to help to determine what will be accepted as an explanation and puzzle-solving solution, as well as to determine as yet unsolved puzzles and their relative importance to the group. In my terms, 
although models help to do this, all of these are functions of the matrix, rather than of individual models. Models are rather the result than the cause of these things and are thus not to be identified with the matrix itself. In my view, models have a specialised role in the sense that they are specific interpretations of given theories in which these theories are true.$^{53}$ So, the formulation of models has to be preceded by factors such as determining the nature of these models, whether they will offer an explanation for whatever problem is being studied, and so on. The disciplinary matrix should in my terms be seen as offering the background against which actions at all three levels of the scientific process take place.

\subsubsection{Paradigms as shared examples}

Traditionally it is thought that scientific knowledge is embedded in theory and rules. A student cannot learn to solve problems before she has learned the theory and the rules for applying it. And then, after having solved many problems, such a student will find it less and less difficult to solve more and more complicated problems. Kuhn (1970: 188) claims that "... at the start and for some time after, doing problems is learning consequential things about nature. In the absence of such exemplars, the laws and theories [the student] has previously leamed would have little empirical content" ${ }^{4}$ He further goes on to say (Kuhn, 1970: 190) that scientists solve puzzles by modelling them on previous solutions. He wants to emphasise that what he refers to as "consequential knowledge of nature" is acquired while learning the similarity relationship and is afterwards embodied in a particular way of viewing nature rather than by laws and rules. He (Kuhn, 1970: 191) claims the verbal statements of laws taken by themselves to be "virtually impotent" ${ }^{54}$ and stressed that what actually results from the use of exemplars is something like Polanyi's "tacit knowledge" which is learned by doing science rather than by acquining rules for doing it. Kuhn (1970: 194) argues that claiming that rules and the ability to apply them are acquired from exemplars implies that there are, already at that stage, alternatives in the following sense. We might, he (ibid.) continues, "have disobeyed a rule or misapplied a criterion, or expenimented with some other way of seeing. [And those] are just the sorts of thing we cannot do." I would qualify this remark by adding 
that those are things scientists cannot do until after they have started making observations and thus taking part in the scientific process. Then they start abstracting data from real systems, constructing conceptual models, and maybe in the end, formulating theories, ahways directed by factors like research and personal goals and working from within a specific disciplinary matrix.

The only way in which we can have scientific contact with the world (that is, with systems in reality) is through actions involving selection, abstraction, and generalisation, which are always executed within some theoretical framework or disciplinary matrix, and are always teleological in character in the sense that these abstractions are made in order to theorise eventually about a specific aspect of some real system relevant for certain context-specific reasons. So in this sense there are indeed no absolute rules and laws guiding us towards the expression of our knowledge, because of the way our encounters with reality are structured. And, in this sense, it is indeed only by constructing various models of reality that we leam "consequential things about nature".

Thus, the way in which models function in a model-theoretic interpretation of the process of science has some of the features that Kuhn ascribed to this second use of the notion of "paradigm". Models can be seen as the basis for solving "puzzles" in the sense that they are actually constructed in the first place, when they form part of the process towards theoryformulation, to do just that. Also, when considering the implementation or application of theories, already established models of theories may lead scientists to new interpretations of these theories, or to amending the existing models in order to offer "better" explanations for the problem the theory is addressing. To claim however - as Kuhn does - that modeis replace explicit rules as besis for puzzle-solving is maybe taking matters a bit far. It is indeed not possible to find absolute laws or rules dictating the construction of models (whether during the process towards theory-fomulation, or afterwards, when the theory is applied), simply because these constructions are 50 context- and theory-specific. But, the fact that models operate as a link between theories and systems in reality, and that they have an essential interpreting role to fulfil in the process of science, is at least a significant feature of 
scientific practice, if not a rule or a law.

As far as Kuhn's claim that models offer scientists a specific way of viewing nature is concemed, that is indeed the case in a model-theoretic interpretation of the scientific process too (although the broader motivation for a specific world view is taken to be offered by a specific disciplinary matrix). Both in the process of theory-formulation and during the application of theories, models are constructed and employed in accordance with a certain view of reality, and aftenwards, these models exist in a sense as an affirmation of these views. See Chapter 5.

\subsubsection{Conclusion}

The problem with Kuhnian revolutions is that they imply more than an epistemological shift, they require an ontological shift that necessitates discontinuity. Kuhn's "different" or "incommensurable" wordds address both the problematic issue of the invariance of (the order of) nature and that of the neutrality of science (or of the language of science). And that is at least one of the reasons why incommensurability has proved to be such an arresting notion - it concerns the status of both of the two poles of scientific realism.

Kuhn (1970: 111) states that "[e]xamining the record of past research from the vantage of contemporary historiography, the historian of science may be tempted to claim that when paradigms change, the world itself changes with them", although he qualified that later (in the Postscript) by saying that it is not "the world" that changes with a change of paradigm, but rather it is that scientists afterwards work in a "different world". He (Kuhn, 1970; 121) wishes to argue against the Cartesian belief that "what changes with a paradigm is only the scientist's interpretation of observations that themselves are fixed once and for all by the nature of the environment and of the perceptual apparatus" (ibid.). In this sense of course, Priestley and Lavoisier both saw oxygen, and Aristotle and Galileo both saw pendulums, although in each instance, they differed in their interpretation of what they had seen. ${ }^{55}$

Now, since The structure... Kuhn has often referred to the process by which later meanings are produced from earlier ones as a "process of language learning". By 1990 he 
had however found this metaphor too inclusive and had started concentrating ${ }^{56}$ instead on the meanings of restricted classes of terms. These terms are taxonomic or kind terms, like "dog", "cow", "gold". They have two characteristic properties:

- They are identified as kind terms by virtue of lexical features such as taking the indefinite article. ${ }^{57}$

- They are subject to what Kuhn refers to as the "no-overlap" principle - no two terms of this kind may "overlap in their referents unless they are related as species to genus" (Kuhn in Tauber, 1997: 233). For example, no dogs are also cows, a bangle is not a ring, and so on. Encountering a cow that is also a dog, would mean, in terms of this rule, that part of a taxonomy will have to be redesigned, and not that it is simply the set of category terms that has to be broadened or enniched.

To be able to describe reality at all, it seems obvious that in these terms, then, some kind of lexical taxonomy should already be in place. Moreover for communication to be possible, shared taxonomic lexicons have to be in place, because if different communities have taxonomies that differ in a certain local area, then situations may occur where statements made in the one will not be expressible in the other. Kuhn (ibid.) claims that the only way to bridge such a gap would be to breach the rule of no-overlap, and so incommensurability becomes a kind of untranslatability. It would be possible, though, for members of one community to learn the taxonomy employed by members of the other, but that would not mean that they would be able to translate terms from one taxonomy to the other, and, moreover the cost of bilingualism is that the particular community in which discourse is occurning at the time, has to be kept in mind throughout, to escape re-instating the original threat to communication. In the scientific context, Kuhn (in Tauber, 1997) makes it clear that periods of revolution will, in terms of the above, then be episodes which require local taxonomic change. All this is to say that there are times in the development of science when fundamental change in taxonomic categories is involved (for example, think of the Copernican and Ptolemaic taxonomies).

The interesting thing, in this context, about a model-theoretic interpretation of the 
process of science is that it can support changes of this kind, without subscribing to a discontinuous view of scientific progress. It seems obvious that there are "degrees" of incommensurability in the above sense - surely the "gap" between the Aristotelian view of science and the Einsteinian one is bigger than that between the Newtonian and the Einsteinian. In other words, it seems as if the nature of episodes of progress depends very much on the specific paradigms or disciplinary matrices involved. And, it is this aspect of the scientific process that may become a lot clearer if the mediation of models between theories and aspects of reality as a kind of magnification of scientific change is acknowledged, because the "decelerating" influence models have on this entire process helps to highlight the different factors of change and their different nuances in different situations. Kuhn's emphasis on the directing role that paradigms play in science's processes is thus affirmed in a model-theoretic account of science, although in such an account the (extremely) slow pace of change of paradigms (in terms of disciplinary matrices) does not necessitate Kuhnian incommensurability. ${ }^{58}$ Given the overarching nature of disciplinary matrices, a change in disciplinary matrix might indeed be a revolutionary event, but the continuity models ascribe to science's processes is usually sufficient to render these events less interruptive than they are perhaps portrayed in Kuhnian terms.

The best way in which to interpret incommensurability in model-theoretic terms is perhaps then to acknowledge the - very gradual and slow - change of disciplinary matnix in the total process of science, but under the following conditions. The incommensurability should be understood to be about change in methodology, interpretation of observations, and general application of scientific knowledge. In other words, incommensurability may be more characteristic of model change than it is of change in disciplinary matrix. One may without real difficulty acknowledge that it would be difficult for Niels Bohr to work within the Einsteinian deterministic disciplinary matrix and get the same results as easily as he did, working in the context of the Copenhagen interpretation of quantum mechanics. However, Bohr and Einstein (Sachs, 1988) often had discussions about their different approaches. It might be that the more steadfast nature of disciplinary matrices that accounts for their slow 
change rate renders the conditions, detail of methodology, and implications of results of separate models less incommunicable as some believers in naive interpretations of incommensurability seem to claim.

Kuhn wants however to stress that, more than merely interpretational and methodological change, revolutions also result in change of disciplinary matrix, the theoretical and meta-theoretical background against which the scientists are working, and everything that goes with that. In model-theoretic terms, however, revolutions take place only in cases of disciplinary matrix change, and not in cases of model change. The phenomenon of incommensurability should thus not necessarily be linked to revolutionary changes. It is possible to have two different models of the same theory within the same disciplinary matrix, while different meta-theoretical orientations do not prohibit scientists from understanding each other.

It seems - especially in later writings - as if Kuhn's objective with arguing about the variable sensory experience of scientists differently placed in history and differently placed with respect to alternative paradigms that form the "different worlds" in which they practice science, is still to somehow recover the "one world". These kinds of "different" worlds, however, have to do with science and its practice. It does not directly have anything to do with Nalure, the "one" world, or however one decides to refer to it. It is, as Kuhn (1970: 121) puts it, "the nature of the environment" and the "perceptual apparatus" that "fix" observations. It should be noted though that interpretations of observations can never be fixed - they can be refined, amended, sharpened, and changed in whatever way, at any minute in any number of ways for any number of reasons. Secondly, the "nature of the environment" and the "penceptual apparatus" that shape interpretations of observations, are part of the disciplinary matrix within which the observations are made as well as the specific "view of the world" it offers.

Reality and our observations of certain of Nature's features are two different things. Nature and its features - such as gravitation - are independent of scientists and their actions, while observations of aspects of reality are not. It is important to distinguish between 
Nature and science; the one given, the other constructed. The always present danger of blurring the conceptual boundaries between Nature and science sometimes ends in a conflation of Nature and science, caused, it seems, by science's directedness towards reality. And this somehow implies, it seems - in a social constructivist kind of way - that science will have some kind of transforming influence on reality. That is the problem: science may change our conceptions of reality (think of the role of background information in shaping these images). However, the features of Nature that we call gravitation have always been related to the features we call motion, position, mass, and acceleration and will always do so. The fact that Newton formulated a very workable, successful version of these relations does not change this in any way. Newton's formulation enabled - and still in limited domains enables - us to understand and employ the phenomenon of gravity and its manifestations, but our understanding will not change the working of these features - except in our models of it, in the "artificial environments" Cartwright likes to speak of.

This is no great disaster though, except if one takes this to mean that scientists have no knowledge about Nature. But it does not mean this, because "having knowledge of Nature" means exactly that abstractions are made from what is presented to our faculties of knowledge (however one wants to fill that in) and manipulating these abstractions conceptually and rationally. Science is the product of scientists' efforts to give a rational account of empirical observations and their implications. ${ }^{59}$ Thus, the way some real system is will not itself be influenced by theory-change. Kuhn himself stated (in Tauber, 1997:243) that it is groups and their practices that constitute "worlds" (interpreted as the products of paradigms or disciplinary matrices) and that science is one of these "practices-in-the-world".

Therefore change in the disciplinary matrix from which scientists work may be triggered or influenced by theory-change, although this kind of change takes place even more slawly than theory-change itself. Think, for instance of Newton's method and how it differs from Cartesian mechanics, especially as far as the use of mathematics is concerned. ${ }^{\infty}$ Descartes had tried to derive basic physical laws from metaphysical principles, while Newton insisted on basing his theorising on a careful examination of reality. ${ }^{61}$ Finally, this kind of change may 
- and often does - lead to a change in the way in which scientists view reality, simply because different aspects of the system in reality they are studying are emphasised because of the change in purpose and method indicated by the matrix change.

The old debate between scientists aiming to "save the appearances" by "superimposing" mathematical relations on phenomena, and those who find explaining exactly why the phenomena are "there" more important, seems somehow to be lurking behind a lol of the issues raised both in this chapter and in Chapter 5 . Einstein spoke in this sense of "principled" and "constructive" theories, and considered his General Theory of Relativity as a principled theory in so far as it met the requirement of visualisability only after formulation. Quantum mechanics' development, however, was preceded by visual or pictorial interpretations, although the requirement of visualisability was rejected later on in formalising a number of basic quantum mechanical equations. Visualisation plays an interesting role in the search for atomic structure. The atomic model described by Rutherford in 1911 was closely analogous to the solar system. Some of Maxwell's theories of a more classical nature however gave rise to objections against this model. Bohr tried to eliminate these in 1913 by combining classical and non-classical approaches. Sommerfeld later elaborated Bohr's model and the analogy with the solar system was retained in the Bohr-Sommerfeld model. ${ }^{62}$

But, by the 1920's Pauli and Heisenberg were rejecting this pictorial atomic structure. Pauli remarked (Sarlemijn \& Spamaay, 1989: 7) that scientists should not try to shackle (the structure of atoms with their preconceived opinions, but should rather adjust their concepts "in line with experience". In a famous paper, written in 1925, Heisenberg stressed that his basis for theoretical quantum mechanics is founded only on "the relationships between quantities which are in principle observable" (ibid.: B). The Copenhagen interpretation of quantum mechanics supports the same approach: it is sufficient for the formalism adequately to establish the connection between the experimental results - no visualisation or physical interpretation of formal calculations are needed. A bit later, however, Heisenberg adopted a different attitude and began to search for physical interpretations that would "fit in" with matrix mechanics. This shows how the way chosen to solve a problem - which is in the first place 
detemined by the relevant disciplinary matnix — may influence scientists' methods and goals - whether to strive towards "saving the appearances", or to have as objective to be able to show the connection between scientific conceptualisations and aspects of reality only after theory formulation has been initiated or even completed.

Scientific method should thus ideally provide a model-dependent model-modifiable strategy, because such a strategy offers - within a realist context - the possibility of modifying or amending our existing theories in the light of further research. The continuous nature of science is also confirmed, since the methodological principles of a strategy like this will themselves depend on the theoretical picture provided by currently accepted theories. Both our new theories and the methodology by which we develop and apply them depend upon previously acquired theoretical knowledge. And this fact about the cumulation of scientific knowledge - as well as science's vanous relations to reality - can best be supported and explained by a model-theoretic - reslist - conception of scientific knowledge.

To clarify the distinctions and similarities between a model-theoretic account of scientific theories and the various non-statement approaches to this issue, in the next chapter some of the main non-statement approaches will be briefly discussed. 


\section{CHAPTER THREE: VARIATIONS ON THE NON-STATEMENT VIEW OF SCIENCE ${ }^{63}$}

\subsection{Introduction}

In Chapter 1, the Introduction, I have discussed the differences between the statement and the non-statement approaches. The advocates of the statement approach depict scientific theories as axiomatised deductively closed sets of sentences within some appropriate syntactic system, and discuss the "empirical interpretations" of these theories in terms of some set of correspondence rules or bridge principles. The defenders of the nonstatement approach, on the other hand, do not view the formal formulation of scientific theories in some appropriate language as the most useful characterisation of "theories". Rather, they depict these "theories" in terms of sets of mathematical structures that are the models of the theory in question. They do not, however, escape the usual realist questions in this way, the problem is simply pondered in a different context with the help of a set of more workable - in the sense of adaptability to different situations - tools.

In what follows I shall discuss a few of the main non-statement programmes offered by current philosophy of science. I focus on the non-statement approach to science since its defenders' acknowledgement of the role of mathematical models in science's processes is of paramount importance to my model-theoretic view of science. Although I have the notion of a scientific theory as a deductively closed set of sentences in common with the defenders of the statement approach to science, the rest of their (absolutist) views do not feature in my approach at all, and therefore I shall not discuss their approach in any more detail.

\subsection{Patrick Suppes's set-theoretic approach to science}

Suppes offers one of the first viable alternatives to the "received (statement) view" of scientific theories and so brings about a radical turn in philosophy of science. Other than the structuralists who stress the use of formal semantics and meta science to appeal to the structural aspects of theories, Suppes finds the axioms of set-theory sufficient, and claims mathematics, rather than meta-mathematics to be the language of science ${ }^{64}$

Since to define a class of set-theoretic structures of which a theory is true (relative to 
these structures) it is irrelevant to know really what the theory is about, he seems to be not overly interested in the problem of identifying or limiting the intended applications of scientific theories in the classic "statement" sense. I try to emphasise as often as possible that there are at least two kinds of truth relation to be examined when asking questions about the "truth of a theory". As I have pointed out in Chapter 2, the first kind of truth relation that theories typically enter into is purely formal in the sense of Tarskian satisfiability, and here, obviously, it is the case that the content of theories is not necessarily a deciding factor. The second kind of truth relation (between models of theories and empirical models depicting the behaviour of phenomena in real systems) is also formal in this sense, but also far more complicated, given that these relations are the relations determining empirical adequacy and all that it implies. And, obviously, in the second case, that "about which" theories are, becomes extremely relevant.

According to Suppes (1967: 57) the problem with the statement approach's co-ordinating correspondence rules is that they do not in the sense of modern logic offer an adequate semantics for the axiomatic calculus of the theory. Wojcicki (in Humphreys, 1994: 127) explains that "... if for the logical positivists the right way to define an empinical theory $T$ was to define a set of axioms from which all the other sentences valid in $T$ are logically derivable, Suppes suggests that to define $T$ is to define a set-theoretical predicate that denotes all the set-theoretical structures [semantical models] of which T is true in the Tarski sense". Suppes does not so much emphasise the non-statement approach versus the statement approach though. He rather stresses the advantages of analysing empirical theories within a settheoretical framework rather than a meta-mathematical one ${ }^{65}$, and (Suppes, 1954: 244) writes:

... Why axiomatise?, I may briefly say that axiomatisation is one constructive way of obtaining the sort of intellectual clarity and precision for which philosophers are always striving with respect to the foundations of the various sciences. Unfortunately a good many philosophers seem to labour under the misimpression that to axiomatise a scientific discipline ... one needs to formulate the discipline in 
some well-defined artificial language. ... this kind of linguistic viewpoint is, in my opinion, seriously in error, and the predominance of this attitude has perhaps been one of the major reasons for the lack of substantial positive results in the philosophy of science. ... Luckily we can pursue a programme of axiomatisation without constructing any formal languages. The viewpoint I am advocating is that the basic methods appropriate for axiomatic studies in the empirical sciences are not metamathematical (and thus syntactical and semantical) but set-theoretical. To axiomatise the theory of a particular branch of empirical science in the sense 1 am advocating is to give a definition of a set-theoretical notion, such as that of a system of classical particle mechanics (see McKinsey, Sugar, and Suppes (1953)), or that of a system of rigid body mechanics (see Adams (1959)), or that of a system of Mendelian genetics (see Rubin (1954)).

The class of structures (systems) under consideration is thus described by giving one "generic" structure, with parameters, which can be specified to deliver all the systems in the class. Suppes (in Morgenbesser, 1967: 60) acknowledges this when he points out that one of the simplest ways in which to provide an extrinsic characterisation of a theory is to define the intended class of models of the theory; and then asking if the theory can be axiomatised, merely comes down to asking if a set of axioms can be stated such that the models of these axioms are precisely the models in the defined class. He (in Morgenbesser, 1967: 61, 62) remarks however that "... the problem of intrinsic axiomatisation of a scientific theory is more complicated and considerably more subtle .... Fortunately, it is precisely by explicit consideration of the class of models of the theory that the problem can be put into proper perspective and formulated in a fashion that makes possible consideration of its exact solution."

And, in model-theoretic terms, even more positively, such consideration of the class of models of a given theory shows the continuous character of science (see Chapter 2). The underdetermination of theories by models and their underdetermination by data are more problematic from a non-statement point of view perhaps, precisely because from such a point 
of view the issue of underdetermination seems untouchable (and so insoluble), given the nonstatement aversion to theories as linguistic entities, so that the notion of a theory as some kind of overarching organising notion does not really exist for defenders of this view. In a model-theoretic approach underdeterminalion is more "natural" and even somehow forms part of scientific progress. ${ }^{66}$ I shall elaborate on the meaning of underdetermination in modeltheoretic realist terms in Chapter 5.

Suppes addresses the philosophically problematic relations between empirical systems and theories (i.e. my "second set" of interpretational relations) in terms of a hierarchy of models that focuses on the complex nature of the experimental process. ${ }^{67} \mathrm{He}$ (Suppes, 1954:243) already points out very early on in his work that progress in foundational studies of philosophy of science requires distinction between theory and experiment, since the reconstruction of the experimental stage of science is rather more problematic in comparison to the theoretical stage which may be axiomatised "quite easily" with the help of set-theoretic predicates. He (Suppes, 1954:246) wants to provide philosophy of science with "... a kind of algebra of expenimentally realisable operations and relations" and emphasises that discussion of the empinical interpretations of the primitive notions for certain defined notions of some empinical theory imply interpretations of quantilative notions, which recessitates some systematic theory of measurement. ${ }^{83} \mathrm{He}$ is not interested in the classic notion of absolute objective truth, nor is he interested in the kind of framework offered by the instrumentalists, rather he wants to speak about truth in terms of modem statistical decision theory.

Thus, one of the most important issues in Suppes's philosophy of science is the emphasis he puts on the "experimental stage" of science ${ }^{69}$ Empinical interpretations of the primitive notions for certain defiried notions of some empirical theory are interpretations of quantitative notions, which necessitates some systematic theory of measurement, as already mentioned.

... the point of a theory of measurement is to lay bare the structure of a collection of empirical relations which may be used to measure the charactenistics of 
empinical phenomena corresponding to the concept. Why a collection of relations? From an abstract standpoint a sel of empinical data consists of a collection of relations between specified objects. For example, data on the relative weights of a set of physical objects are easily represented by an ordering relation on the set; additional data, and a fortion an additional relation, are needed to yield a satisfactory quantitative measurement of the masses of objects ${ }^{n}$ (Scott \& Suppes, 1958: 113).

Thus, as far as the co-ordinating principles or bridge principles of the statement approach are concemed, Suppes stresses (in Morgenbesser, 1967:62) that the practice of testing scientific theories is a much more complicated issue than is implied by the usual comment about these issues. $^{70}$ I agree with this, but I do not see the philosophical need for tuming almost exclusively to the statistical methodology to examine these relations that Suppes (in Morgenbesser, 1967, and also Suppes, 1969, Suppes, 1989, and Suppes, 1993) insists on. ${ }^{71}$ I think that for the purposes of philosophy of science, it is sufficient - and a more philosophically challenging prospect, I might add - to look to the various model-theoretic relations involved, and to be able to point out all of (or as many as possible of) the factors involved in these connections. ${ }^{72}$

Suppes and Dana Scott in their article Foundational aspects of theories of measurement (1958) ground the foundational analysis of measurement in general model theory. Suppes (in Morgenbesser, 1967:58) points out that the essential characteristic of a theory of measurement is that it can study (in a precise way) the transformation or development of "qualitative observations" into the "quantitative assertions" characteristic of the more theoretical stages of the scientific process. He approaches this problem in terms of representation theorems, mainly because he views the models of the theory and the models of the data (see below) to be of different logical types: "Given an axiomatised theory of measurement of some empinical quantity such as mass, distance, or force, the mathematical task is to prove a representation theorem for models of the theory which establishes, roughly speaking, that any empinical model is isomorphic to some numerical model of the theory. The 
existence of this isomorphism between models justifies the application of numbers to things. ... What we can do is to show that the structure of a set of phenomena under certain empinical operations is the same as the structure of some set of numbers under arithmetical operations and relations" (Suppes in Morgenbesser, 1967:58). ${ }^{73}$ Although I would read "conceptual model" for his "empirical model" and "empirical model" for his "numerical model", this is essentially my view of the "verification" of the models of scientific theones too. In my approach it is however not necessary to use a separate language — from the one talking about the content of a theory's conceptual models - to talk about the empinical models of theories - although of course it can be done, and then Suppes's use of representation theorems will become applicable too. ${ }^{74}$

Suppes (1954: 245 ) sets out the various stages of formulating a set-theoretic predicate for (or axiomatising) a particular branch of empirical science as follows:

- In the beginning some kind of statement of what other theories are assumed (e.g. in axiomatising rigid body mechanics, one would assume the standard branches of mathematics and particle mechanics) is needed.

- Then the "primitive" notions of the theory are listed, and their set-theoretic nature (in particle mechanics, notions like "set of particles", the "interval of elapsed time", the "position function", the "mass function", and so on) is indicated.

- The set-theoretic definition can then be completed by listing the axioms which have to be satisfied, because one will then be able to examine the deductive consequences of the definition. Obviously one of the main tasks here is to rationally reconstruct within set theory the standard theorems of the branch being studied. One will also then be able to ask some of the questions of modem mathematics that have obvious implications for the structure of empirical theories, such as questions concerning the formulation of representation theorems ${ }^{75}$ which may be linked for instance to studies directed towards the problem of reduction between theories ${ }^{75}$.

Then finally, one will be in a position to give an empirical interpretation of the axiomatised theory, which will have to take the complexity of the entire experimental enterprise into 
account.

Suppes thus articulates a more complex stratified view of the relations between models of theories and systems in reality, than I do in this thesis. However, I too go to great lengths to point out the elaborate sophistication of the manoeuvres needed to find the possible links between particular systems in reality and certain models of a theory being examined at a given time. He (Suppes, 1989: 25) wants to show that the study of the relations between (empinical) theories and their data demands a study in terms of a hierarchy of models of different logical type ${ }^{77}$. He (Suppes, 1960: 297) stresses the clarifying role of the set-theoretical notion of model in experimental design and the analysis of data: "The maddeningly diverse and complex experience which constitutes an experiment is not the entity which is directly compared with a model of a theory. Drastic assumptions of all sorts are made in reducing the experimental experience ... to a simple entity ready for comparison with a model of the theory" (ibid.).

Suppes sees the empirical relation between a conceptual model (of a given theory or class of systems) and a system in reality as a highly articulated, composite relation, with an articulation which depends upon the experimental or observational situation in question ${ }^{78} .1$ am in complete agreement with this view, but for my (philosophical) purposes I collapse this complex relationship to a much simpler relation, indicated by "empirical adequacy" (in my terms). This simple relation results in fitting the empirical data - however elaborately extracted from the physical system, and subsequently formulated conceptually, i.e. mathematically - into the relevant conceptual model of the theory in question (i.e. the relevant conceptualisation of the empincal data in question forms a substructure of the conceptual model in question). A simple example: Observations over time deliver 113 different spatial positions $\left(x_{1}, y_{1}\right),\left(x_{2}, y_{2}\right), \ldots,\left(x_{113}, y_{113}\right)$ for the planet Neptune in the $x-y$-plane (of the planets in our solar system) of a coordinate system centred on the sun. These are the data. All 113 points lie on the (near-) ellipse with its uncountably many points which is the conceptual model of the orbit of Neptune (which in its turn is part of the conceptual model of the solar system, in which the - Newtonian or Einsteinian - theory of our solar system is 
true). What is suppressed or collapsed here is the process of distilling the data $\left(x_{1}, y_{1}\right),\left(x_{2}\right.$, $\left.y_{2}\right), \ldots,\left(x_{113}, y_{113}\right)$, which is a process which involves theories, models, and practices relating to telescopes, the human eye and visual system, light, movement of the earth, clocks, and so on, and so on.

Suppes (1989: 27-29) argues that the fundamental theory and descriptions of apparatus are two extremes of hierarchy - in between are the models of the theory, the models of the experiment, and the models of the data. He (in Morgenbesser, 1967: 62) emphasises over and over again that to be able to "connect" experimental data to a relevant theory, the data have to be put through a "conceptual grinder", which refers to this conceptual hierarchy he sets out from the "raw" observations to the final "fundamental" scientific theory. The theory of the experiment is the definition of all the possible realisations of the theory that is the first "step down from the abstract level" (Suppes, 1989: 28) of the fundamental theory. A possible realisation of the theory of the experiment is a model of the theory if the experimental conditions are satisfied. Models of the experiment represent experimental data in canonical form, but when is a possible realisation of the data a model of the data? Suppes (1989: 29) remarks again that an answer to this kind of question requires a "detailed statistical theory of goodness of fit", since models of the data should incorporate "all the information about the experiment which can be used in statistical tests of the adequacy of the theory" (Suppes, 1989: 31), which means that he (Suppes, 1989: 32) restricts the models of the data to those aspects of experiments which have a parametric analogue in the theory. ${ }^{79}$ In these terms, I would express Suppes's model of the scientific development of a theory (Suppes, 1989: 31) as follows:

Fundamental theory

Models of the fundamental theory

Theory of the experiment

Models of the experiment

Models of data

Experimental design 


\section{Ceteris paribus conditions}

Suppes (1989: 32 ) characterises the ceteris paribus conditions at the bottom of the table as "every intuitive consideration of experimental design that involves no formal statistics", which I presume refers to the context in which the concrete as-yet-untranslated-into-data "first" observational activities are camied out. Note that in a model-theoretic account of theories the ceteris paribus conditions are at play in the formulation of the theory and not at the level of dealing with data. He seems to think, as Cartwright does, that the closer to reality we get, the more of these clauses we need, while I claim that only by a suspension of these clauses can we move to the more specific levels of the scientific process. Thus although I do not deny the idealised character of our conceptual models or even of the images of real systems our empinical models present, I claim that these "idealisations" are not so much a result of ceteris paribus clauses as simply of the nature of scientific actions. (More about this in Chapter 4.)

A last point to note is that he (Suppes, 1989: 32) makes it clear that in analysing the relations between theories and experiments, difficulties encountered at any of the levels from the level of models of the theory to Suppes's last identified level of ceteris paribus conditions reflect problems or weaknesses in the relevant experiment, and not in the relevant "fundamental" theory. I agree, although it seems obvious that errors may also occur in the formal construction of the models of the theory, and also, that problems encountered in creating these constructions may indeed point to problems in the structure of the fundamental theory ${ }^{80}$.

Suppes (1989: 34) concludes: "One of the besetting sins of philosophy of science is to overly simplify the structure of science. ... What I have attempted to argue is that a whole hierarchy of models stands between the model of the basic theory and the complete experimental experience. Moreover, for each level of the hierarchy, there is a theory in its own right. Theory at one level is given empirical meaning by making formal connections with theory at a lower level." I am in complete agreement thus far, but again, my version of a model-theoretic view of the structure of theories can fully accommodate this kind of hierarchy. There is a principle of transitivity at work here - accommodated by a model-theoretic 
account of theories - that does the same philosophical work as Suppes's intricate comments on measurement theory and the role of representation theorems.

Take Newton's laws of motion and his law of gravity applied to our solar system again. Such a model (i.e. a model of our solar system) will be described by an uncountable set of sentences which, i.a., describes every position at any time of every planet in question on an elliptical curve. I claim there exists a transitive connection between experimental data and some model of the theory, since the data offer "pieces" of the model by means of some "experimental theory" in the sense that the theory of the experiment "translates" observations into data (or models of data), which can be then possibly linked with (i.e. embedded into) some model of the theory. If a scientist is looking at a planet through a telescope, the theory of the telescope translates those observations into data, and these data give the position of say Mercury at a given point in time. But this is exactly what the empirical models of Newton's theory offer (in this context), since a conceptual model of the solar system offers here the positions of all the planets at specific times. The data thus do depict certain relations valid in models of the theory.

\subsection{The structuralist programme}

Stegmüller ((1976), (1979)) places the structuralist programme in the "non-statement" tradition, because its main methodological principle is to view "theories" as structures, or sets of structures (in the standard set-theoretic sense), in the place of sets of statements. Sneed (in Balzer, Moulines, Sneed, 1987: 86) writes "... our point of view is not that of a philosopher who is puzzled by questions like how it is possible to obtain knowledge about 'the phenomena'. Our attitude is much more descriptive, and if we look at what is 'given' for an empirical theory we take the point of view of that very theory - in contrast to the 'absolute' point of view of the philosopher". This refers to the fact that the structuralists are more concerned with making apparent the logical form or structure of empirical theories than with their actual content. That is why their aim is to create "logical reconstructions" of empirical theories, rather than worry about the "usual philosophic issues to do with science and reality" 
(Sneed, 1983: 350) ${ }^{81}$. Also, Sneed (1971) and Stegmüller (1976) claim this approach can best handle Kuhn's condition for membership of a particular scientific community in terms of "holding" a certain theory, although a community's beliefs about the subject matter of that theory is not fixed over time.

Structuralists typically depict a scientific theory as a "... conceptual structure that can generate a variety of empirical claims about a loosely specified, but not completely unspecified, range of applications" (Sneed, 1976:120). This view is closely related to what I hold too, except for the fact that a scientific theory to me still is a linguistic expression - and an essential component of science - that may be interpreted by a set of (conceptual) structures (i.e. models in which the theory's sentences are true). 1 also take these models (and not the theory itself) to be the "generators" of "a variety of empirical claims" about a certain range of applications of the theory.

The structuralist programme dates from the early sixties and is essentially a development of Patrick Suppes's view. The programme originally started (Sneed, 1983:350) as an attempt to describe more precisely the empirical claims of theories with considerable mathematical apparatus. Since the early seventies Wolfgang Stegmüller, with his colleague Joseph Sneed, assisted by Wolfgang Balzer, Ulises Moulines and others, started refining this approach. The structuralist approach is a meta-theoretical approach to scientific (empinical) theories that essentially focuses on the nature of scientific theories, interrelations between theories (especially reduction, equivalence, and approximation), and theory progress or evolution. During the last two decades in various disciplines formal reconstructions of empirical theories into structuralist form have been carried out by the supporters of the programme, such as physics (Sneed, 1973), psychology (Suppes, 1969, 1989), and neuropsychology (Suppes, 1989).

Before I briefly discuss the main tenets of their programme, a comment on their view of the role of language, given their "non statement" approach. In An architectonic for science (Balzer, Moulines, Sneed, 1987: 17) it is claimed that "... we [Balzer, Moulines, and Sneed] believe ... that in the study of the structure and development of empirical science language 
has not a big role to play. This belief is mirrored in our concept(s) of an empinical theory which will not contain a language as an explicit part. It would ... be a serious misunderstanding to say that therefore we have dispensed with language altogether. ... What is left open in our account is the way in which sentences are formed out of basic symbols, variables, and other logical symbols. But such formation rules do not play any role in empirical science ...". They (ibid.) go on to point out that a species of structures totally describes the "non-logical" vocabulary of a given theory (i.e. its individual constants, predicate constants, function constants and their anities).

I agree that the last point may be true, but I have a problem with the first remark claiming that the formation rules of sentences (of theories) do not form part of empincal science and that this is justification enough for the structuralists to leave it out of their analysis of empirical theories. Of course these rules (like the rules of mathematics) do not form part of empinical science itself. However, (again like mathematics) they provide the means to formulate and communicate the conceptual structures, and they do form part of a philosophical analysis of science, since part of such an analysis would be to examine the structure and meaning of scientific theories as linguistic expressions. ${ }^{82}$ Retaining the notion of theories as linguistic expressions is the only way I can see in which to formulate the class of models of a theory in "one" formal expression. Even the structuralists have to employ language to describe the classes of structures they have in mind, albeit the mixture of natural language and mathematical symbolism usually employed by scientists and mathematicians, and of which a formal logical language is a (meta-) mathematically amenable stylisation. ${ }^{\text {ag }}$ Also, the linguistic expression of a theory offers different ways of "controlling" the multiplicity of models of one theory in the sense that amending the axioms of a given theory can logically strengthen the theory such that it "shrinks" the class of possible models (via boundary or initial conditions), or the theory can "shift" to a different class of models, such as happened when Einstein added the "cosmological constant" to his equations (theory!).

Joseph Sneed, in giving an exposition of what he sees as the subject matter and nature of philosophy of science (Sneed, 1976: 121), explains that the structuralist claim is that "... 
everything interesting a scientist of science might want to say about the products of science can be said within [a] conceplual [structural] framework. More precisely, it can be said within an ontology of scientific theories ... with sufficiently ingenious relations among these entities". He remarks (Sneed, 1976: 116) that philosophy of science in general is about setting out a "clear, coherent conceptual framework" in which the various sciences can formulate their empirical claim5. However, he (ibid.) continues that the "science of science" he is thinking about should be a social science, since its main subject matter are the communities within which these empirical claims are formulated. The coming-into-being, development, and goingout-of-existence of these communities as well as of their products (empinical scientific theories) should, it seems then, all form part of this subject matter. ${ }^{84}$

The basic elements of theory identification as set out by the structuralist programme may be summarised as follows (note that structuralists claim that all these components can be precisely explained in purely structural - i.e. set-theoretical - terms). "Theories" are taken to consist of (classes of) models in the Tarskian sense of formal semantics, l.e. a model of a theory $T$ is a possible realisation in which all the sentences (or at least a set of given axioms) of that theory are satisfied. ${ }^{\text {s5 }}$ The identity of a "theory" is first and foremost given by a class of models, which we may call $M$ (following Stegmülier and his colleagues) ${ }^{66}$ The models are determined by a given set of axioms (the "tautologies" of the theory), but the structuralists claim these axioms to be secondary to the determination of the identity of a theory, since any set of axjoms may be chosen just as long as it is satisfied by the same set of modeis, $M$.

The structuralists try to "fit" models to theories, since they do not acknowledge the theory as a linguistic entity to start off with. A defender of a model-theoretic account of science works towards the formulation of a theory as a linguistic expression, and then, in applying the theory, starts off with the theory as a linguistic entity and tries to construct models in which that particular theory will be true.

However, although it is true that in the structuralist view the specific set of axioms in question does not really play a primary role in the identification of the theory, distinguishing 
between two different kinds of axiom does. In the literature, these two types of axiom are usually referred to (Moulines in Schurz \& Dom, 1991:317) as framework conditions ${ }^{87}$, which are mainly the accepted body of theonies, or background knowledge, or paradigm within which scientists work, and "proper" axioms which are taken to be substantial empirical laws. In the example of Newtonian mechanics, Newton's Second Law is a "proper" axiom, while the (implicit) condition of the differentiability of the position function would be a framework condition. The framework conditions define the basic notions about the structure of each of the fundamental notions of the theory, i.e. the "base set" (Balzer, Moulines, Sneed, 1987: 5) of the theory; while the "proper" axioms state the law-like relations between these basic notions, i.e. "what conditions have to be satisfied for a possible candidate to really be a structure of this kind?". The latter are the "fundamental laws" (Balzer, Moullines, Sneed, 1987: 19) that "connect" all the terms of a theory in one "big formula".

Structures determined only by framework conditions are called potential models, the class of which may be denoted by $M_{p}$, or $M_{p}(T)$, while structures fulfilling both the framework conditions and proper axioms of a given theory, are called actual models, the class of which may be denoted by the familiar $M$, or $M(T)$. Obviously $M(T)$ is a subset of $M_{P}(T)-$ so that $M \subset M_{p}$. Methodologically speaking, clear distinction between these two classes of models is necessary in any logical reconstruction of a theory ${ }^{\text {Bg }}$. Thus the set-theoretic predicates determining the set $M_{F}(T)$ are defined by statements about the set-theoretic properties of the base set of the theory, and typifications and characterisations of basic relations. The determination of $M(T)$, on the other hand, relies on the specification of the laws (axioms) identified in the theory as well ${ }^{\text {ag }}$.

Also part of the determination of the identity of a theory is a distinction between the theoretical and the non-theoretical terms of the same theory, the interrelationships ("constraints") between models of the same theory, as well as the intertheoretical links between models of different theories (concerning different sets of potential models).

Advocates of the structuralist programme take $\left\langle M_{p}, M\right\rangle=K$ (Moulines in Schurz \& Dom, 1991:319, and Balzer, Moulines, Sneed, 1987:36ff.) to be the (conceptual) "theory-core" of 
a particular theory. The core $K$ plus the class of intended applications, call it $I$, form the simplest set-theoretic structure that may serve as a logical reconstruction of an empirical theory. $K$ and $I$ are called theory elements. $K$ is a purely formal mathematical structure and it says "something" or is "about" the class of intended applications. More complex theories are "built" of theory-elements that are linked or related in certain ways. In summary, the notion of a theory core is expanded to include the following elements (Balzer, Moulines, Sneed, 1987: 37):

- conceptual framework conditions,

- empirical laws, i.e. the "proper axioms",

- "constraints" describing connections or relations between different applications of a particular theory, and

- intertheoretical links (such as the relations of reduction, approximation, and equivalence) describing links between a particular application of the theory in question and other different theories represented by different theory elements.

- Also part of the theory core is the class of partial potential models $M_{P P}$ that consists of a subset of $M_{A_{1}}$ the class of potential models, that can be interpreted independently of the theory in question. Partial potential models are thus characterised in terms of a theory-relevant theoretic/nontheoretic distinction among the components of the class of potential models. More about partial potential models a little later in this section.

- An empirical claim - also part of the theory core - is associated with a particular theory element in terms of the part of the content of that theory element which forms the class of partial potential models that is "compatible" with the laws, constraints, and intertheoretic links associated with the particular theory element in question. This claim is simply the claim that the particular intended application of the theory in question is in $K$, i.e. in $\left\langle M_{\rho_{1}} M\right\rangle$.

An obvious motivation (that both realists and anti-realists would agree on I should think) for empirical theory construction surely is the (successful) application, in one way or the other, of that (empirical) theory. That is why claiming that we know what an empirical theory looks 
like if we know its core, is not completely correct. We also need to have some information on the nature of its intended applications. Structurally speaking, then, if we take $I$ as the set of intended applications of a given empinical theory identified by a specific given $K$, we have to know the nature of the elements of $I_{\mathrm{r}}$ as well as the extension ${ }^{90}$ of $I$. Note again that cores of theories and the applications of theories together - i.e. $M_{P,} M$, and $I$ - are the "material" out of which empinical claims may be formulated.

Now, the elements of $I$ are taken - by the structuralists - to be not "simply the 'real things', independent of any conceptualisation, to which the theory is supposed to apply" (Moulines in Schurz \& Dom, 1991: 319) ${ }^{51}$, but rather systems, which are nothing else but structures, that present us with ways of "... conceptually carving up reality in pieces and putting these pieces in certain relationships" (Moulines in Schur \& Dom, 1991: 320). Thus, we can take a system, $s$, to be a structure of the form $\left\langle A_{1}, \ldots, A_{m}, R_{1}, \ldots, R_{n}\right\rangle$. The important issue here is to determine the relationship between the class of potential models, $M_{p}$, and a particular intended application, s. Obviously, for a system s to be an intended application of a theory, it has to be an element of the set of potential models, $M_{\rho}$, in the core of that theory, and thus a necessary condition for the determination of a set $I$ of intended applications of a theory given by $K=\left\langle M_{p}, M>\right.$, becomes $I \subset M_{p}^{2 q}$. Sneed (in Humphreys, 1994: 196) helpfully points out that $I$ should be seen as the "totality" of potential data the theory in question is supposed to account for.

All right, but exactly what does this subclass ( $I$ look like? How big is it? To be able to understand the structuralist answer to this question, let us think a bit about the nature of the relations between $I$ and the class $M$ of actual models in $K$. We cannot simply assume that $I$ $=M$ since it is entirely possible that any empirical theory might have applications that are unwanted $^{\oplus 3}$, for whatever reason. The implicit result of multiple models of empinical theories entailed by the depiction of theories in terms of classes of models ${ }^{94}$ lies in the problem of identifying "empincally uninteresting" models (or potential models) so that they may be discarded (as soon as possible). As this is a problem defenders of the statement approach also face, it is especially interesting to see how the structuralists face up to it. Balzer, 
Moulines, and Sneed (1987: 23) offer an explication of the application of a theory to a certain (intended) range of phenomena in terms of $M_{p}$ and $M$. They first point out that this issue is related to a distinction between conceptualising a certain range of phenomena and making an empirical assertion about them. For my analysis of the scientific process this is also important, since my analysis is done in terms of a conceptualising stage (leading up to the formulation of the theory in question) and an applicative or interpreting stage (following the formulation of the theory).

So we do not want $M$ to be a subset of $I$, since there will probably always be elements in $M$ that we do not want in $I$. Moulines (in Schurz $R$ Dom, 1991: 321, 322) sets out three possible ways to describe the nature of the relations between $M$ and $I$ :

- (i) $I \subseteq M$

- (ii) $I \not \notin M$, but $I \cap M \neq \Phi$

- (iii) $I \cap M=\Phi$.

Case (i) is the ideal case, where the relevant theory is a complete success, capturing all the intended applications. Case (ii) presents us with a partially successful theory, and, of course, the bigger the inlersaction between $I$ and $M$, the more successful the theory will be. Case (iii) presents us with a theory that has no intended applications among its actual models, which, of course is a completely meaningless theory,

Moulines (in Schurz Dorn, 1991:321,322) points out that in terms of the methodological evaluation of empinical theories it is evident that the intersection between $I$ and $M$ needs to be defined or specified as precisely as possible, although the structuralists stress that there is no purely semantic answer to the question conceming this intersection. ${ }^{.0}$ Any kind of approach to this issue has to be preceded by what they term "pragmaticdiachronic considerations" (Moulines in Schurz \& Dom, 1991:321), because of the fact that for every given theory core, $K$, there has to exisl a scientific community that will use the theory identified by the core in "real life". Because $I$ is dependent on the scientific community within which the theory under consideration has been constructed or will be applied, the structuralists refer to the class of intended applications as a "genidentical" (Moulines in 
Schurz \& Dom, 1991:322) entity. ${ }^{97}$ The relationship between the scientific community and the pair $K$, the core (identity) of the theory in question, is philosophically important, because intended applications are taken — by the structuralists but not by me - to be part of and internal to the theory concept itself, and not somehow external to the theory. Baizer, Moulines, and Sneed (1987:38) claim that without this class it will be impossible to know the empirical content of the theory. They (ibid.: 38) put the structuralist case as follows:

We consider [the class of intended applications] to be a part of the identity of a theory because without it we would have no way to know whether we are dealing with an empirical theory at all. Take the case of an advanced scientific theory, where quite a few abstract terms expressed in mathematical language appear, and let us ask whether by just considening the theory's formalism we would be able to tell which part of the world the theory describes, or for what purposes the theory is useful. ... we cannot tell. For, even by assuming that the formalism is adequate for describing some part of the world, we should be able in general to go over to quite different phenomena described by the same formal means. This is indicated by a well-known theorem of logic, namely, that structures isomorphic to models of a theory are again models of that same theory. ... Thus, in order to know what a theory is about, we have to include an informal description of its intended application, as a part of the identity of the theory in question. ${ }^{26}$

The relationship between the set of intended applications and a given scientific community can vary in the sense that the same community may use completely incompatible cores. On the other hand, different communities may use the same core class and then the relationship between intended applications and cores will be completely different. This is possible because we can define (from the intended nature of these applications) the domain of intended applications of a certain core $K_{i}$ associated with a specific scientific community, as a particular subclass of the class of potential models, $M_{p r}$ for which the scientific community in question wishes (intends) to show (either by observation, experiments, or calculation) that it is also a subclass of the class of actual models, $M$, of the theory in question. "Strictly 
speaking [of course], I may not be characterised as a class in the precise sense of set theory since it is not determined by purely extensional means. ... The domain $I$ remains always within $M_{p}$ but its precise limits within $M_{\rho}$ change as the skills, knowledge and interest of [the scientific community] as a whole change" (Moulines in Schurz \& Dorn, 1991: 324).

This has however already been pointed out earlier by Suppes, and as I have pointed out above, not even the original formulators of some scientific theory can know in advance what will happen to their theory - i.e. in which models it will be interpreted. In my terms, this is a matter to be determined via the empirical models of the theory about the construction of which nothing can be said beforehand. The only thing that can be said about a scientific theory's "identity" is the remarks I made about the role of the "intended models" in the developmental stage of theories. This is no problem though, since given the nature of scientific knowledge, the nature of these applications simply has to be open-ended in this way. ${ }^{99}$

A common criticism against the structuralist programme - which can perhaps i.a. be seen as a product of or reaction to the post-Kuhnian philosophical atmosphere in the late sixties, which was the time in which the structuralist ideas found a wider audience - is that the defenders of the programme view empirical theories in purely mathematical terms. Moulines (in Schurz \& Dom, 1991: 314,315 ) gives a very apt answer to comments of this kind, which, I think, should be taken very seriously. He (ibid.) writes:

If I say that I am going to speak about a set of pigs, then, of course, I am going to speak about 'something mathematical', since a set of pigs is not a pig (an empirical object) but a set (clearly, a mathematical object). This does not mean that I am saying there are no pigs in the world or that the pigs themselves are mathematical entities. Similarly, when structuralists say that an empinical theory is to be conceived of as a particular kind of structure consisting of sets of structures, then, in a trivial sense, they are saying that an empinical theory is a mathematical entity, since, in general, structures consisting of sets of structures obviously are mathematical entities. This, however, does not at all imply that empirical theories 
are being put on the same footing as purely mathematical theories, nor that they only deal with mathematical objects, nor that 'there is no reference to reality'.

In a sense, 1 agree and add that thus elements of sets may be viewed - rather unproblematically - as mathematical representations of real (empinical) objects. However, in this text, Moulines (in Schurz \& Dom, 1991: 315) goes on to make the claim that structuralists in general seem to view as their "link" with reality, but, that, I claim, is really much more negative since it goes against the very spirit of their model-theoretic approach. First, the claim: "At least part of the models that constitute an empinical theory according to structuralism consists, at least in part, of sets of empirical objects. This one can read on the first pages of any introductory exposition of structuralism" (ibid.). Well, I have never been able to see how a real object can be an element of a mathematical entity. He should have said "at least some of the elements of the models that constitute an empirical theory ... represent, at least partly, certain empirical objects".

I believe that the failure of the structuralist programme's defenders to regard the relationship between a model (or a set of models) of a theory and some real system quite in the sense of a model-theoretic interpretative relation, may result in uncertainty concerning their realist sympathies. That such an interpretative relationship exists is denied by Moulines's (ibid.) claim that at least some of the sets of models of a theory are (consist of) empirical objects. He (Moulines in Schurz \& Dorn, 1991: 317) continues to say that "[i]f the theory is to be called 'empirical', at least some of the [domains] of at least some of its models will be such things as sets of physical bodies, or space time points, or persons, or states of mind, or wares, or written texts, or any other sort of empirically detectable entity which nobody would call a 'purely mathematical entity'. That's the way 'reference to reality' comes in[to] structuralism. As simple as that". Well, if everything was this easy, should not somebody perhaps consider telling those philosophers who persist (stubbornly it seems, in the face of the structuralist solution to their problems) in worrying about the problems of scientific realism?

Balzer, Moulines and Sneed (1987: 23) offer the following explication of the application 
of a scientific theory to a certain (intended) ${ }^{100}$ range of phenomena in terms of the set of potential models and the set of actual models of the theory. They write:

When confronted with some given 'data' or 'phenomena' we might want to use a theory $T$ to 'understand' them, to 'explain' them, to 'predict' them - in short we might want to apply $T$ to these data. To do this, the first thing we try is to conceptualise the domain $I$ of data in terms of $\mathrm{T}$, i.e. [we] ... use the concepts appearing in potential models of $\mathrm{T}$ to refer to $I$. We create a potential model of $\mathrm{T}$ for $I$. This is the more 'conceptual' aspect of the application of a theory. The next step is to make an assertion about $I$ in terms of $\mathrm{T}$ - an assertion with empinically testable consequences. We then assert that $I$ satisfies the fundamental laws of $\mathrm{T}$, which, of course, only make sense if $I$ has already been conceptualised in terms of T. In other words, we make the empirical assertion that the potential model considered is also an actual model of $\mathrm{T}$. This empincal assertion can be either true or false. If it turns out to be true, we can say that we applied $\mathrm{T}$ to $I$ successfully.

More specifically, Sneed (1976) and his colleagues (Balzer, Moulines \& Sneed, 1987) describe the set of (intended) applications of an empinical theory in terms of some set of partial potential models (i.e. theory-independent subsets of the set of potential models), and an empirical claim associated with the core of the theory in question. Such an empirical claim states that the set of partial potential models that satisfies the conditions set by the laws, constraints, and intertheoretic links of the theory in question, is indeed in $K$, the theory's core. If one recalls that the class of partial potential models represents subsets of the class of potential models, the above explication in terms of intended applications remains the same in general. This finer distinction of the class of potential models focuses on those theoretical terms that are specified by other theories and not by the theory in question. This implies that the part of a theory that may have relations to reality cannot be determined only by the theory itself. Sneed (in Eaizer, Moulines, Sneed, 1987: 86) remarks that the class of partial potential models represents what is "given" for a particular theory in terms of surrounding theories.

Why is this distinction between so-called "T-theoretical" and non-theoretical terms 
necessary? Let us first look again at the way in which the defenders of the structuralist programme see theories connected to their empirical claims. The structuralists' answer to these questions become very complex and extremely technical since they formulate these answers in terms of constraints, inter-theoretic relations, and partial potential models. Most simply put, as noted above, cores of theories and the applications of theories together form the "bricks" out of which empirical claims may be formulated.

Theories consist of these basic "theory-elements" ${ }^{101}$ which have the ability to construct various "theory-nets" (which are basically more complex theories than the original one in question) from the relevant theory's original elements. A theory-net consists of a "specialisation" of the basic theory element. This specialisation corresponds to an empirical claim about the range of intended applications offered by the theory element in question. Thus the net as a whole corresponds to a non-basic empirical claim about the whole range of intended applications the theory has to offer. The problematic part of this analysis of theories lies in the overlaps between applications in various theory elements. These theory elements are linked by the interrelationships between models of the same theory (referred to as "constraints" in the structuralist programme). And, it is these problems that may be solved - or so the structuralists seem to think - by distinguishing between theoretical and non-theoretical terms. In this sense the definition of the core of a theory is expounded (Sneed, 1976: 123) as follows:

$K=\left\langle M_{P}, M_{P P}, M, C>\right.$, where

- $M_{p}$ is the set of potential models of the theory, as in the above, but with the understanding that these models are models of the entire conceptual content of the theory, including theoretical components;

- $M_{P A}$ the set of partial potential models, is the set of all models obtained by excluding the theoretical components from the conceptual body of the theory in question ${ }^{102}$;

- $M$ is still the set of actual models, since it depicts the set of possible models of the "full conceptual apparatus ${ }^{n}$ of the theory that satisfy certain laws formulated in terms of theoretical components; 
- $C$ is the set of constraints on $M_{P}$, and captures the notion that different applications of the same theory-element are interdependent ${ }^{103}$ in the sense that values of a function in one application of the theory may not be used without taking account of the values of that function in other applications - thus, constraints single out certain admissible combinations of potential models ${ }^{104}$; and

- inter-theoretical links are depicted as follows: T-nontheoretical terms may be also or only determined by means of other theories which do not presuppose $T$ at all, so that the problem of communicating information from these theories to theory $T$ becomes problematic - Balzer, Moulines, and Sneed's answer (1987: 58) is - not too surprisingly - to link this with the interpretation of the set of partial potential models in the following way: "The information [from theories different from $T$ that we want to transfer to T] consists of data which are obtained in the course of some determination of a term which is non-theoretical in T. Clearly such transfer contributes to the interpretation of $M_{p p}(T)$. It is part of the determination of the meaning of the terms occurring in T's partial potential models, and therefore it is an essential component of T itself. ... [This leads to the introduction of] ... intertheoretical links which represent the transfer of data from theories $T^{\prime}$ to theory $T .^{n^{105}}$

Sneed (1976: 124) ${ }^{106}$ claims that a theory-element core is used to make empirical claims in the sense that a subset of $M_{\rho p}$, call it $A(K)$ (ibid.), is selected such that theoretical elements can be added to each of its members in such a way that it yields a subset of the set of actual models $M$ (this means that each member of the subset of $M_{p p}$ will satisfy the theoretical laws of the theory). ${ }^{107}$ The empinical claim that the particular theory element is thus making, is that descriptions of phenomena that actually occur is indeed a part of the theory core. In other words, if we have a theory-element $E=\langle K, I\rangle$, where $K$ is the elaborated theory core above, and $I$ remains the set of intended applications, then the claim that $E$ is making is that $I$ is an element of the subset $A(K)$ of $M_{p p}$. That means that the theory-element core $K$ narrows down the set $M_{P P}$ to the subset $A(K)$, thus restricting the possible models of the theory (containing only non-theoretical components) such that the result is $I$. This "narrowing down" is done via 
constraints and intertheoretical links ${ }^{100}$. Balzer, Moulines, and Sneed (1987: 87) point out that the assumption that the intended applications of $T$ have the structure of its partial potential models is the "most economical and most natural" assumption to make. Thus we should assume that $I(T) \subseteq M_{p o}(T)$. There will, of course, still be unwanted applications, even if we take $M_{p \rho}$ as the set of all possible applications of the theory, but these gentlemen (1987: B7) claim it is enough that "[w]e can say something precise [after all], namely that an intended application is a partial potential model, but we cannot be precise about every feature of intended applications". ${ }^{109}$

In model-theoretic terms - as I shall discuss in Section 5.5 - the notions of intertheoretical links and constraints are mostly addressed in terms of underdetermination via the various models of a particular theory. The "narrowing down" of the set of applications of a theory is best done by amending the axiorns of the theory itself, although the various relations possible between theories and models of the same theory as well as models of other, possibly related, theories also may be applied in this sense. As far as the last remark above is concerned, also in a model-theoretic account of science is it not possible to depict all the possible (intended) applications of a specific theory, as has already been pointed out often.

\subsection{The semantic approaches of Beth, Van Fraassen and Suppe}

Bas Van Fraassen developed a semantic approach to philosophy of science by buliding on the work of Evert Beth ${ }^{110}$, in which physical systems are depicted in terms of their possible states. This position was further developed by Frederick Suppe ${ }^{111}$. The foundational claim of this approach is that any scientific (physical) theory is taken - by scientists themselves to have many alternative linguistic formulations. (Think of the Lagrangian and Hamiltonian formulations of classical particle mechanics.) Theories thus cannot be identified with their linguistic formulations. Suppe (1973:130) claims that it is rather the case that "... scientific theories are extra-linguistic entities which are referred to and described by their various linguistic formulations ... [thus] theories are to be constructed as abstract structures which serve as models for the sets of interpreted sentences [that] constitute their linguistic 
formulations (i.e. that they are meta-mathematical models of their linguistic formulations), where the same structure (theory) may be the model for a number of different, and possibly inequivalent, sets of sentences or linguistic formulations of the theory. ${ }^{112}$ Here, then, is a more radical approach than either that of Suppes or the structuralist programme in the sense that the theory is identified, as it were, with the notion of model. A theory is a model to the defenders of this approach, they do not merely talk about discarding the linguislic features of theories in logical reconstructions, they claim a theory to be "extra-linguistic".

Beth ${ }^{133}$ developed what is referred to as a "state-spaces" view (related to the older phase and configuration space view of mechanics and that of Von Neumann (1955) for quantum mechanics) in three articles (1948/49), (1949), and (1961). Van Fraassen (1970:327), following Beth, believes that the meaning structure of a certain part of natural language becomes suitable for a technical role in some scientific language if it has a representation in terms of a model, in the sense of a mathematical structure. Then the scientific language can be formally reconstructed as an artificial language whose semantics is determined with reference to this mathematical structure or model. Such a language Van Fraassen (1967) calls a "partial or semi-interpreted language". ${ }^{\text {"14 }}$ Note that the "meaning structure" of a part of natural language that may be represented by some mathematical model may here then be described in some appropriate formal language. In a model-theoretic approach the semantic content of the linguistic expression of the theory in some appropriate formal language is determined by the initial conceptual model, but also, the linguistic theoretical expression is then interpreted by other conceptual models during the applicalion stages of the theory ${ }^{115}$, which implies nothing more than that the "meaning structure" of the linguistic expression is then represented yet again by other (or the same) mathematical structures. However, this is not what Van Fraassen and Beth really claim. To them the model is the mechanism that may determine the semantics of the formal language in which the theory may be formulated, as are my initial conceptual models. However the theory itself remains a non-linguistic entity, since nowhere do they mention the possible interpretation of the theory in terms of other mathematical structures in the model-theoretic sense. ${ }^{116}$ 
Now, in Beth's approach, the notions of a "physical system" of a theory and the various "states" in which this system can be at given times, are foundational. These notions are however easier to understand if some of Suppe's notions are introduced first. The specific class of phenomena that the (linguistic) formulation of a theory is meant to characterise, is called the intended scope of the theory. Theories do not characterise these phenomena in their complexity, though. Suppe (1973: 131) gives as illustration the fact that classical particle mechanics characterises mechanical phenomena as if they depend only on the abstracted position and momentum parameters, while actually various other "unselected" parameters usually also influence the phenomena. Thus a theory's characterisation describes what the relevant phenomena would have been like had the abstracted parameters - those the theory's formulation focuses on for whatever reason — been the only parameters influencing them. This is essentially what happens both in my onginal intended models and in the later conceptual models of a given scientific theory. More about this a little later in this section.

In this sense, theories may be said to characterise physical systems, because they are about the behaviour of certain abstract systems in the sense that this behaviour is dependent only on the parameters selected by the theory. Physical systems are relational systems whose domains consist of states, and relations and laws ranging over these states. ${ }^{117}$ Van Fraassen (1970: 330) states that the function of a law in Beth's approach is to describe the behaviour of the physical system with which the theory is occupied at the time in terms of its possible states, its normal evolution through time, and its behaviour in interaction with other factors. ${ }^{118}$ These laws thus indicate which states are physically possible for the various physical systems; and they also determine which combinations of states are so-called theory induced physical systems (notion explained below) and which are not. "Thus the relations of the theory determine all and only those sequences which are the behaviours of physical systems in the class of theory induced physical systems" (Suppe, 1973: 133).

The selected parameters abstracted from the phenomena can wholly describe the behaviour of physical systems, and so they are called the defining parameters of the physical system. The values of these parameters are physical quantities which may be determinate 
or statistical (Suppe, 1973: 131). A set of simultaneous values for the parameters of a physical system is a possible state of the system. Note that any physical system is an an time in exactly one of its possible states, although that state may change over time. The behaviour of a physical system is given in terms of these state changes. In this way, the behaviour of a system is the system's history and each physical system has a unique sequence of states (in the deterministic case) or a set of possible sequences of states with associated probabilities (in the statistical case) that it assumes over time. Each physical system is characterised fully by a specification of the possible states it can assume and the sequences of states it assumes over time.

The class of causally possible physical systems for a theory is the class of physical systems which correspond in the following way to causally possible phenomena $P$, within the theory's intended scope: any $P$ in the theory's intended scope corresponds to a (causally) possible system $P^{\prime}$ such that $P^{\prime}$ is what any causally possible phenomenon $P$ would have been were the idealised conditions imposed by the theory met and the phenomenon $P$ influenced only by the selected parameters. Obviously then, one of the tasks of a theory is to describe the class of causally possible physical systems for the associated theory. This is done by the theory describing a class of physical systems known as the theory induced class of physical systems, such that this class is identical to the class of causally possible physical systems.

A theory then is empirically true if the theory induced class of physical systems and the class of causally possible physical systems for the theory are indeed identical. Testing of theories involves determining whether this identity in fact exists between these two types of classes of systems and is usually done in statistical terms.

As far as the semantic content of the theory formulation ${ }^{119}$ is concerned, Van Fraassen (1970:328) writes that the "set of states of some physical system are represented by elements of a certain mathematical space, called the state-space. ${ }^{120}$ Apart from the statespace, these theories use a certain set of parameters - referred to in the above - to characterise the particular physical system. This yields the theory's set of elementary 
statements about the system in question. These are initial and boundary conditions which are part of the relations detemining empinical adequacy in my terms. These statements are such (ibid.) that each elementary statement $U$ formulates a proposition to the effect that a certain physical magnitude $m$ has a certain value $r$ at a certain time $t^{12 t}$ The truth of such a proposition $U$ always depends on (or is relative to) the particular state of the system at that time - in some states $m$ will have the value $r$ and in some states it will not have that value. ${ }^{122}$

A "satisfaction function" determines whether the system's actual state is represented by an element of the mathematical structure consisting of all the relevant state-spaces of the theory or not: "The mapping $h$ [of the theory] (the satisfaction function) ... connects the statespaces with the elementary statements, and hence, the mathematical model provided by the theory with empirical measurement results. ${ }^{123}$... The exact relation between ... [elementary statement $U]$ and the outcome of an actual experiment is the subject of an auxiliary theory of measurement, of which the notion of 'correspondence rule' gives only the shallowest characterisation" (Van Fraassen, 1970:329). ${ }^{124}$ A description of a set of state-spaces plus the satisfaction function are thus offered in the place of the statement approach's axioms or postulates concerning the "primitive" symbols of the scientific language. ${ }^{125}$ / agree, although l offer the entire model-theoretic stratified process of science in the place of these postulates.

Now if a physical system is in the class of theory induced systems, then the domain of the physical system will be a subset of the domain of the theory and the sequence of states of that system will be one determined by the theory's relations (laws). These physical systems are meant to be replicas of the actual systems in reality, and so by describing the physical systems the theory "... indirectly gives a counterfactual characterisation of the actual phenomena" (Suppe, 1973: 131). Also, it may happen that theories give an idealisation of some physical system - Suppe's (1973: 131) illustration again is from classical particle mechanics. These kinds of systems are "isolated systems with dimensionless point masses interacting in a vacuum". Such idealised physical systems are still abstract replicas of phenomena, but with the additional feature that certain idealised conditions (such as being isolated systems of dimensionless point masses) are imposed on these systems which actual 
phenomena can never actually meet. Thus (Suppe, 1973: 139) "[0]nly some of the propositions which are true of the theory will be true of a particular physical system in the class of theory-induced systems, but every proposition true of a physical system in that class will be true of the theory". If a theory is empirically true, the semantic relations holding between propositions in the theory-formulation language and the class of causally possible physical systems for the theory will be exactly the same as those holding for the theoryformulation language and the class of theory-induced physical systems, Moreover, every proposition in the theory-formulation language which is true of a causally possible physical system will be true of the theory.

A few remarks on this semantic approach to scientific theories in terms of a modeltheoretic account of these matters. In model-theoretic terms the intended scope of a theory is indeed the "class of phenomena" the (formulation of) the theory is meant to characterise. It is simply the case that within the latter kind of account we usually speak of real systems rather than phenomena or classes of phenomena. Also, in the latter approach, a theory can do no more (as Nancy Cartwright so delights in pointing out) than characterise the real system it means to describe as it would have been had the abstracted parameters of the theory been the only ones influencing the system in question. (1 shall discuss this "idealised" or "open-ended" feature of scientific theories more deeply in Chapters 4 and 5.)

Each possible state of a physical system - in Suppe's terms - might be viewed as a conceptual model in model-theoretic terms, seeing that a possible state of a system is given in terms of a simultaneous set of values for the parameters of the theory in question. The theory-induced physical systems would then perhaps best be viewed in terms of the empirical models of a model-theoretic account. The reason for this is that the class of causally possible systems tums out to be systems in which the idealised conditions set by the theory have been realised, influenced only by the selected parameters of the theory, and that a theory is said to be empirically true in Suppe's terms if this class of systems is identical to the class of theory-induced physical systems. The relation of empinical adequacy between conceptual and empirical models of some theory in model-theoretic terms is then very close to this relation 
of identity between causally possible physical systems and theory-induced physical systems. This becomes even clearer if we take into account that the "satisfaction function" of Suppe's semantic framework determines whether the actual state - i.e. the causally possible state — of a physical system is represented by the mathematical structure representing the theoryinduced physical systems.

\subsection{Ronald Giere's naturalistic approach to science}

Giere (1985:75) agrees with Van Fraassen that the logical positivists' (statement view) pre-occupation with the linguistic structure of scientific theories obscures the important role models in which those theones are true, have to play in the scientific process. He, however, does not waste much time in pursuing any of the semantic categories of reference and meaning in the way Van Fraassen does (via his notion of elementary statements yielding semi-interpreted languages). He (Giere, 1985:77) states clearly that he "... will simply ignore such issues. ... the theory of science need not wait on the development of adequate general theories of meaning and reference to proceed. We need not know in detail how general terms such as mass come to be associated with terms in an abstract mathematical structure. We know that it can be done because it is done" along. I have stressed that, because of the complex and changeable nature of these issues, questions concerning experimental design, measurement theories, and criteria determining the "fit" of some model to a system in reality, are best left to science itself to answer. I do however think philosophy of science has something to say about these issues, at least as far as showing their place in the structure of science as a whole (i.e. in the relations between the possible empirical and conceptual models of some theory), and their implications for the structure of scientific theories in particular (i.e. their possible reference to real systems).

Giere sees himself as a supporter of the non-statement view of theories, preferring Beth's state-space approach to Suppes's set-theoretic one. He states (Giere, 1994: 277) that he interprets the model-theoretic approach to imply that "... theories include two sorts of linguistic entities. Some are predicates, which may have an elaborate internal structure, as, 
for example, the predicates 'pendulum' or 'two-body Newlonian gravitational system'. Others are statements of the form ' $X$ is $P$ ' in which $X$ refers to a real world system and $P$ to one of the predicates, as in the statement, 'The earth-moon system is a two-body Newtonian gravitational system'n. These remarks might be viewed in terms of a model-theoretic approach's relations in its conceptual models - as the "predicates" Giere refers to, and such an approach's empirical models - Giere's "statements" concerning the "real world". In general, the structure of a theory consists, according to Giere (1994:277), of a 'family' of models or predicates, where the linguistic structure corresponding to a predicate is a definition instead of an axiomatic system (as in the traditional statement approach).

Giere addresses these issues in terms of "theoretical models" (the models (or set of models) created by defining a certain real system), and "theoretical hypotheses" which are statements picking out similarities between theoretical models and some system in reality. ${ }^{126}$ He (Giere, 1983:271) makes it clear from the start that theoretical models (as definitions of systems in reality) have no empirical content. They may, however, be used to make claims about reality via the theoretical hypotheses that identify elements of some theoretical model with elements of real systems and then claim that the real system exhibits the structure of the model in question. Giere (1984:11) acknowledges the idealised nature of models ${ }^{127}$ by stating (Giere, 1985:79) that a theoretical model is not "a faithful replica in all detail" of the object modelled. He (Giere, 1984:12) goes on to explain that theoretical models also come in various degrees of specificity, but points out that no such thing as a maximally specific model exists. This kind of model is always relative in the sense that it is a model of a designated type; thus a model is always an idealisation of reality. Giere $(1984: 12)$ also refers to the underdetermination of a theory by its models: "There are many ways of filling in a highly nonspecific model to achieve a highly specific version of that model. The relationship between non-specific and specific models, therefore, should not be confused with the relationship between general and particular, as in the relationship between general laws and particular instances" (ibid.). ${ }^{128}$

He (Giere, 1983:272) stresses though that a theory is not simply a general model. He 
(ibid.) blames scientists for thinking that theories have empinical content and accuses them of using the term "theory" to refer to a "more or less" generalised theoretical hypothesis asserting that "one or more specified kinds of system fit a given type of model". He (Giere, 1985:78) accuses the logical positivists of conflating two separate functions of a theory, namely to offer general interpretations of theoretical terms such as "mass"; and to provide the means of identifying particular instances of these terms. The two "furictions" of theories that Giere refers to might be respectively viewed in model-theoretic terms as the "work" conceptual models and empinical models do.

Giere, it seems however, wants actually to study "how we as human beings use abstract models in describing particular objects in the real world" - which reminds somewhat of Wartofski's approach ${ }^{128}$. He does this not by means of that favourite realist notion of approximate truth, nor in terms of Van Fraassen's (1980:9, 45ff.) notion of approximation in the sense of one model of a class of models fitting the real system, but rather by mearis of a particular notion of "similarity". Giere $(1985: 80)$ claims that theoretical hypotheses assert that " $\mathrm{t}$ the designated real system is similar to the proposed model in specified respects and to specified degrees ${ }^{n} .{ }^{130} \mathrm{He}$ adds that the precision associated with any hypothesis is always less than or at most equal to the precision of the measurement techniques employed at the time. ${ }^{134}$ Giere $(1983: 269)$ thus finds the rationality of science in the testing of "highly specified theoretical models against empinical data". ${ }^{132}$

Giere (1983: 272) remarks that theoretical hypotheses can also vary from having a very simple form to being a very complex type of claim, and that (Giere, 1984: 13) they reflect the level of specificity of the corresponding theoretical model. The simplest form of a theorelical hypothesis is a claim that a particular identifiable real system fits a given model. In my approach this would correspond to the claim that the empinical model obtained from the real system sits isomorphically embedded in a given conceptual model, making the latter empirically adequate for the real system (relative to the procedures delivering the empirical model), However, claiming for instance that our solar system is a Newtonian particle system (with a suitable set of initial conditions) involves the whole mechanical theory of the 
Newtonian system. He (Giere, 1984: 13) notes that theoretical hypotheses can thus be more or less general in the sense of including more or fewer real systems of various kinds. "Consider, for example, Newton's theory of celestial mechanics, Mendel's theory of inheritance, or the plate tectonic theory of the earth. On this account ... the difference between a 'hypothesis' and a 'theory' may be largely honorific" (Giere, 1984: 13). Well, in model-theoretic terms this is not the case. The difference in scope between these kinds of theoretical hypotheses rather refers to the kinds of structures it relates to each other. The "truth" relations between a conceptual model and its theory will necessarily be more general in scope than those between an empirical model of a theory and one of its conceptual models. It is also worth noting that theories then rather than theoretical hypotheses contain all the different possible histories of some real system that could result from different, but physically possible, initial conditions.

\subsection{Wójcicki's empiricist semantics of science}

Wójcicki (in Humphreys, 1994:125) argues that the coordination of set-theoretic definitions of theories such that the statements of scientific theories can be related to empirical hypotheses (i.e. theoretical hypotheses "decided" by observation) is an extratheoretical issue. Philosophers of science should therefore not attempt to address this issue within their analyses of the structure of science by notions such as the structuralist notion of "intended applications". Wójcicki thus replaces "observability" with "empirical decidability". I agree in the sense that philosophy of science is concemed with matters of empinical decidability, rather than with observations themselves, and also wish to point again to the overlap with and elaboration of Van Fraassen's constructive empincism here. ${ }^{133}$

When turning to the nature of empincal interpretations, Wójcicki (in Humphreys, 1994:131) also refers to the underdetermination of theories by data: "[n]o scientific theory is just true about the empinical systems to which it applies. ... Empirical systems are, to appeal to a Peircean metaphor, 'nebular. They are never fully separable from their environments; moreover they consist of objects whose properties are not uniquely determined and may not 
be exactly such as is required by the theory. Thereby, if a theory applies to any such system it applies to it in an 'approximate' way'. However, as a result of Wójcicki's model-theoretic sentiments, he (Wójcicki in Humphreys, 1994:132) stresses - much in accordance with my own approach ${ }^{134}$ - that it is not so much the issue of approximation and its determination that is important, or the related fact that "... scientific theories dramatically fail to be totally true of the states of affairs to which they refer" that should drive one's notion of truth. Rather, he views the implications of a model-theoretic approach to imply that theories are "partially" or "relatively" true, in the sense of being true "in certain selected respects". And it is such an approach, he - rightly - claims (ibid.) that can indeed account for the ways in which scientific theories refer to real phenomena.

He (Wójcicki in Humphreys, 1994:133) sets out his approach as follows: "An application of an empirical theory $T$, by itself or combined with some auxiliary hypotheses, to an empirical phenomenon $\Pi$ in order to solve a specific problem $Q$ conceming $\Pi$ may require the formation of a theoretical model ${ }^{135}$ (one may prefer to say mathematical moden of the phenomenon...". This implies defining an abstract system $M$ meant to satisfy the following conditions: $M$ is a realisation of $T$, and $M$ is a faithful representation of $\Pi$ under all the respects that are relevant to $Q$.

Thus, if $A$ is the solution to $Q$, then $A$ is factually true, i.e. true of $\Pi$ if and only if $A$ is true of $M$. A theoretical model of $\Pi$ is a model of an aspect $\Pi / Q$ of the phenomenon $\Pi-$ each other problem connected to $\Pi$ may lead to the formulation of a new model $\mathrm{M}^{\text {. }}$. ${ }^{136}$ This notion of a theoretical model is very close to my notion of an intended model. The "truth" of the solution depends an the accuracy and reliability of the steps resulting in the solution offered by theoretical model $M$, and is a matter of statistical analysis of all the steps of the procedure. ${ }^{137}$ In order for the model's equations to have a unique solution, Wojcicki requires that the values of the parameters of the equations should either be established experimentally or be deduced from the available experimental data with the help of hypotheses we consider to be confimed, i.e. the laws of the theory. This points towards an assumption of the same kind of hierarchy of models as Suppes's. ${ }^{138}$ 
I should mention that Wójcicki (in Humphreys, 1994:138) however does not view theoretical models as "the right candidates for the role of intended applications in the AdamsSneed sense $e^{\mathrm{N}}$. According to him the problem lies in their identity as realisations of the theories which are intended to refer to certain empinical systems. ${ }^{139} \mathrm{He}$ (ibid.) allows that, even though theoretical models are not empirical systems, they can be viewed as some idealised representations of these systems. Why then does he not allow for the kind of transitive reference or interpretation relation 1 argue exists, with the theoretical models mediating between their theories and empirical systems? If (theoretical) models may both be realisations of theories and representations of empincal systems, why can they not serve as a semantic link between theories and empirical systems? His acknowledgement (in Humphreys, 1994:138) of the fact that a structuralist intended application may indeed be a structure which may turn out to be a realisation of the theory in question, simply makes his comments on this issue more puzzling. He (Wojcicki, 1979:158), however, views the notion of "semantic model" as synonymous to that of "realisation", so that it does seem as if the problem here is distinguishing between the use of the various terms in certain contexts, especially given that he continues to state that "[c]learly, the same system may happen to be a model for an instance of an empinical phenomenon [my intended and his theoretical model] and at the same time a model for a set of laws [my conceptual model interpreting the theory and his semantic model] which describe the behaviour of that phenomenon (Wójcicki, $1979: 158)$

The problem might lie in the fact that Suppes and a few others want to distinguish between the "theoretical" model and a "semantical" one in a fundamental way, and perhaps Wójcicki simply does not point out clearly enough that someone like Suppes does not assume the two roles that a mathematical model can play in the process of science in the way we do. In this sense, Suppes (1960:291) claims the set-theoretical model — Wójcicki's semantic models and my conceptual models - to be more fundamental than the "physical" one - in Wojjcicki's terms, the theoretical one, and my intended model. I fail to see why the same kind of mathematical model might not be seen as playing both roles at different times of the 
scientific process. ${ }^{140}$

Suppes seems to reduce this interplay to interplay between the semantic model and the theory, which is acceptable as long as one accepts that this "semantic" model may sometimes play the role of Wójcicki's "theoretical" model. Thus, neither is Wójcicki nor am I introducing theoretical models as a separate class of formal models opposed to semantic models - we rather emphasise that the important thing is the stage at which one is creating a model: either "as a method for finding a new theory [theoretical model] or as a test of a given theory [semantic model]" (Wójcicki in Humphreys, 1994: 146) ${ }^{141}$

Wójcicki $(1979: 158-160)$ also points out that the semantic use of the term model is offen confused with the use of the term as a set of mathematical equations describing some kind of regularity in the behaviour of a particular phenomenon. I agree with him that this should be avoided and that the latter use should rather be changed such that it is taken that the meaning of "model" in that sense is "theory". Wojcicki (1979:160) remarks that "[i]t seems more reasonable then, when speaking about mathematical models, to speak about mathematical entities the equations define [and] which serve as abstract representations of the phenomena examined rather than about the equations themselves". This seems to imply that the sets of equations should be referred to as the "theory". ${ }^{142}$

\subsection{Conclusion}

The different relations between theories, models, and systems in reality offered by the various non-statement approaches to the scientific process all offer - among other things - variations on the theme of scientific realism. The model-theoretic tools these views are equipped with seem to offer a very good chance of, on the one hand showing that there are, indeed, such relations, and on the other hand, to define the nature of these relations more precisely than before. A model-theoretic rather than a non-statement (or semantic) approach promises the most at this point since it not only "speaks about" the relations of the models (of some theory) to reality (or rather physical systems in reality), but also about the relations between these models and the theory itself. In this way a truly model-and-theoretic realism 
can be achieved. The non-statement elimination of the theories as linguistic expressions actually do away with half of the realism issue. That is the main point of difference between my approach and the non-statement approaches to science discussed in the above.

There are however among these approaches and mine also quite a few common aspects conceming especially the final "empirical" links between models (of some theory) and real systems. Adams (1959) was the first person whom I know of who, in terms that may be interpreted in a realist way, described an empirical theory in terms of (i.e. as consisting of) two classes of structures: a class consisting of all the theory's "realisations", and a class consisting of all the intended applications of the theory in question. The latter class is merely a class of empirical structures (i.e. physical - or "real" - systems) of which the theory is (expected to be) true. As I see it, the problem is not only then to show that the theory is true of these empirical structures, but also to describe the relations - if any - between the "realisations" of the theory and these more physical structures making the theory true.

Now, as I have said often in the above, these are the two most difficult questions a realist, model-theoretically speaking, has to face. However, these questions can only be answered on the basis of - and analogous to, in a cerlain sense - the (formal) relations between a theory and its conceptual models (which I take to be Adams's "realisations"). In model-theoretic terms the answer to both the above "difficult" questions lies in the claim that a(n empirical) theory is true of Adams's empirical structures, because it is true (formally) in its conceptual model(s) within which we find the particular empirical structure(s) in question to be (isomorphically) embedded.

Most of the non-statement advocates discussed above offer notions conceming the last empirical model-theoretic link between theories and real systems - i.e. notions concerning the idea of empirical submodels embedded isomorphically into some conceptual model(s) of a given scientific theory - that are at least reminiscent of those of a model-theoretic approach:

- Suppes's hierarchy of theories and models expresses in far more detail the more simple modeltheoretic relation of isomorphic embedding at this last "empirical stage" of theory 
development.

- Without a distinction between theoretical and non-theoretical terms, structuralists simply say that a particular intended application is an element of $M_{p}$. If such a distinction is made, they say that a particular intended application belongs to the class of partial potential models, $M_{F F}$, which is formally derivable from $M_{p}$. Sneed and his colleagues link these classes of models (whether $M_{p}$ or $M_{p p}$ - whichever one is applicable) with an empirical claim associated with the core of the theory in question. Such an empirical claim states that the set of (partial) potential models that satisfies the conditions set by the laws, constraints, and intertheoretic links of the theory in question, is indeed in $K$, the theory's core. The role of these empirical claims would, in my terms, be fulfilled by the isomorphic relations between the conceptual model(s) and empirical submodel(s) of a given theory, which - at least partly - are determined by the empinical expressions giving the empirical data in question.

- In terms of Beth's state-space approach the link to reality is given via some satisfaction function between some (mathematical) state-space describing some physical system, and a set of elementary statements concemed with physical measurements. This means that the actual state of a physical system at a certain time may be given by defining some state-space representing the possible states of that system and some satisfaction function, which holds if the actual state of the system (described by some elementary statement) is an element in the domain of the relevant state-space. Suppe claims a theory to be empirically true if the semantic relations holding between the propositions in the theory-formulation language and the class of causally possible physical systems are those that hold in the case of theory-induced physical systems as well. If a physical system is in the class of theory-induced systems, the domain of the physical system will be a subset of the domain of the theory, and the sequence of states of that physical system will be determined by the theory's laws. ${ }^{143}$ The relations delermining the isomorphic embeddings of empinical models into conceptual models of a given theory are fairly close to Beth's "satisfaction" function. Also Suppe's definition of empinical truth 
makes a lot of sense translated into model-theoretic terms - i.e. "empirical submodels" for "causally possible physical systems", and "conceptual models" for "theory-induced physical systems".

- Van Fraassen announces a theory empirically adequate if some model of the theory is such that (real) structures describable in experimental and measurement reports are isomorphic to an empinical substructure of the relevant model of the theory. This is obviously the approach closest to mine. Van Fraassen's empiricism and non-statement sympathies however keep his views still sufficiently "non" -if not "anti" - realistic for me not to subscribe to them unconditionally.

- Giere (1991: 29) views theories as being represented by a family of theoretical models and a set consisting of these theoretical hypotheses that "pick out things ... that may fit one or another of the models in the family" (ibid.). Evaluating the truth of such hypotheses is a matter of statistical methodology (Giere, 1991), since he claims $(1985: 80)$ a real system to "have the same structure as a model" if the system is similar to the model to specified degrees and in specified respects. ${ }^{144}$ This is yet another albeit perhaps a weaker one - version of the model-theoretic isomorphic embedding between empirical submodels and conceptual models of scientific theories.

- Wójcicki (in Humphreys, 1994) speaks of factual truth if the solution offered by some theory is true of the phenomena the theory wants to explain, as well as true in a model of the theory. This model should be a realisation of the theory and should represent the phenomena in question in all relevant respects - which essentially is a shortened version of Adams's original approach. The strong agreement between Wojcicki's and my approaches is illustrated by the following remark. He (Wójcicki in Humphreys, 1994:137) writes: "The fact that formation of a theoretical model presupposes formation of a model of the data as well as the fact that formation of a model of the data can be controlled by the requirement of consistency of the model with the corresponding theory are of key significance for proper understanding of the interplay between the data and the theories, and thus for proper accounting for both the corrigibility of the data and the 
falsifiability of the theoretical claims".

On the whole Suppes's approach seems to me to hold the most promise as far as solving problems concerned with possible relations between theoretical entities, empirical data, and phenomena go. Obviously Van Fraassen's notion of empinical adequacy determined by certain relations of isomorphism is very close to my own ideas on these issues. In my terms, however, proving the existence of relations of isomorphic embedding between empinical models and conceptual models (which incorporate Suppes's hierarchy of models between, at the highest level, theories of experiments, and the notion of experimental design, closest to reality) offers a way - the possibility of which Van Fraassen denies — in which to refer to the contingent and complex relations between real systems and theories via their mathematical models in a precise way. ${ }^{145}$

From this brief summary it is obvious that the reasons why I am not fully satisfied with the non-statement approach to the nature of scientific theories and their links with reality do not lie in their use of the term "model" nor in their interpretation of it, The problem is rather that in a model-theoretic account of science the general terms of the theory are a prerequisite for linking these theories to (systems in) reality. No realism concerning real entities that is still a scientific realism can work without the organising role of the general linguistic terms of the theory.

In this sense an interesting change of direction is offered by Nancy Cartwright's approach to the theories of physics. She is not a non-statement defender, but neither does she really fit into the statement framework. She does retain a (syntactic) notion of a theory, in the sense that she often refers to sets of field equations as theories, but it is not always clear what her views are on the notion of theories as deductively closed sets of sentences. Her continued claims concerning the "falsity" of the fundamental laws contained in scientific theories seem to indicate that, like the advocates of the non-statement approach, she views the role that theories (as linguistic entities) play in the processes of science redundant. Thus, like mine, her account of science has statement and non-statement characteristics and also she addresses the issue of realism in various ways throughout her account. That is why the 
whole of the next chapter is devoted to discuss - against the background of my modeltheoretic account of science - the main points of her approach. 


\section{CHAPTER FOUR: NANCY CARTWRIGHT AND THE LYING LAWS OF PHYSICS ${ }^{146}$}

\subsection{Introduction}

Nancy Cartwright is one of the most influential philosophers currently writing on the role of models in the process of science. Although some of her work comes very close to the model-theoretic interpretation of science that I am offering, there are also serious differences in our approaches. At the end of this chapter I shall claim that my interpretation can incorporate hers, eliminating some of the more serious problems I think still exist in her work, and - especially in Chapter 5, but already in some of the later sections of this chapter - 1 shall show how a model-theoretic account of scientific knowledge has more to offer current realism than either of her simulacrum or causal accounts of science.

Cartwright's main claim is that scientific theories (or rather, the "fundamental laws" which are part of the theories' content) have very little or nothing to say about reality. She argues for this with the aid of two arguments:

- an instrumentalist, anti-fundamentalist, and, mostly, anti-realist, strategy arguing against a "theory-driven" interpretation of the function of models in (philosophy of) science, and - a metaphysical argument offering a hierarchy of causalities, dealing at the highest level with the capacities of real things - representing a "patchwork of laws" - based on a notion of reality as not necessarily being ordered and structured, even possibly being "disunified".

\subsection{Phenomenological and fundamental laws}

Nancy Cartwright claims in How the laws of physics lie (1983) that considening the truth of the (fundamental) laws of physics will force anyone to admit that almost all of these laws are strictly false, i.e. "lie", because they are valid only under certain circumstances or given certain conditions that do not strictly hold in reality. However, it is interesting to note that the implication ["Conditions" $\rightarrow$ "Law"] is (logically) true even if the "conditions" (the antecedent of the implication) are not satisfied. Therefore "inapplicable" would be more appropriate than "lying", which seems to imply "false" in Cartwright's context. 
Her distinction between phenomenological and fundamental laws is centred around the distinction between the particular (concrete) and the general. She claims this distinction to be in the Aristotelian sense of emphasising the richness and vitality of the particular, although it is interesting that she chooses not to refer to the fact that Aristotle saw the richness of the particular only becoming fully realised in the "universal" (or general). My approach is therefore perhaps more "Aristotelian" than hers, because my model-theoretic interpretation of the process of science will be meaningless without the role it ascribes to general statements (i.e. scientific theories), while Cartwright is always trying to get away from the need to assign too meaningful a role to this kind of statement. She quotes (Cartwright, 1983: 9) Boltzmann's equation and the general equation of continuity used by Maxwell in his explanation of the motion in a radiometer as examples of fundamental laws and describes these laws as "general, abstract equations; ... not about any particular happenings in any particular circumstances."147 | do not think that this description of fundamental laws is debatable. What is debatable, however, is whether this necessarily leads to the conclusion that fundamental laws have no links with aspects of the real world.

Phenomenological laws are complex descriptions of actual situations in very specific terms - "what can be confirmed through tests and comparisons with observations are phenomenological laws - comparatively detailed descriptions of concrete situations, which because of their richness in detail, do not have great generality (sometimes called 'low-level' generalisations)" (Cartwright, 1983: 129). Cartwright (1983: 129) claims that it is phenomenological laws that fulfil the "traditional role" of laws in the sense that they describe empirical regularities - which fundamental laws - because they are too general and much too simple - cannot do, since they cannot account for the actually observed variety in the behaviour of objects in reality. Fundamental laws do not have anything to say about "regularities" (constant conjunctions of events in Humean terms), because describing regular behaviour requires more and more complicated descriptions of the situation. The descriptive phenomenological laws thus have less and less generality and they can never be stated without exceptions, while fundamental laws "by contrast, are simple, general, and without 
exception" (Cartwright, 1983: 157).

Cartwright (1983: 55ff.) quotes the universal law of gravitation, Schrödinger's equation, and Maxwell's equations as further examples of fundamental laws, and gives (ibid.: 2) Airy's law of Faraday's magneto-optical effect as an example of a phenomenological law, because Airy's law does not explain Faraday's law (in the way that the more theoretical treatment of it in terms of electron theory by Lorentz does), but rather describes the actual changes in Faraday's dense borosilicate glass as magnetic fields rotate the plane of polarisation of light (while Lorentz's formulation of the Faraday effect appeals to the electron theory, and so has an underlying explanatory content to it, that Airy's law does not have). Other examples of phenomenological laws she discusses (Cartwright, 1983: 55ff.) are the performance characteristics of lasers as specified by their manufacturers, and the phenomenology of fundamental particle interaction, including things like scattering cross-sections. She choses these examples, because, as Alan Chalmers (1987: 83) points out, "[m]easurements of the rotation of the plane of polarisation are related to the strength of the magnetic field that causes it in the way specified by Airy's formula, lasers do perform [mostly!] in the way specified in the manufacturer's instruction manual, and, for example, scattening crosssections of interacting proton beams at some specified energy are reproducible whenever such beams are made to interact". ${ }^{148}$ It seems then that Cartwright claims fundamental laws to be explanatory of the content of phenomenological laws, and phenomenological laws to be descriptive of aspects of reality. Explanation and description are thus done at different levels of the scientific process. This is a very important point, and is also accommodated in my model-theoretic account of science, but it is not a point that necessarily scores any marks for any kind of anti-realism. I shall show that, on the contrary, it is rather a supportive point in a model-theoretic realist account of science.

It is claims like the following about fundamental laws that do not seem to be entirely correct from a model-theoretic perspective - "... fundamental laws ... do not hold for the most part, or even approximately for the most part, and conversely, those laws which are more or less true much of the time are not fundamental" (1989: 174). The unease that such claims 
cause is not necessanily the result of what she says about the nalure of these laws ${ }^{149}$, but rather that they seem to imply that she still believes in some absolute notion of truth. She stresses that fundamental laws can - possibly and at most - explain the content of phenomenological laws by organising or classifying them, and that fundamental laws therefore do not describe the behaviour of real objects in the world. However, as Rueger and Sharp (1996: 95) point out, fundamental laws are in this context still useful to her even though they are not "Irue descriptions" of real objects or their behaviour, precisely because they "organise and classify our knowledge in an elegant and efficient manner" (Cartwright, 1983: 100). She creates the impression in How the laws of physics lie (1983) though, that she might view the fact that fundamental laws only serve to organise and summarise real phenomena as a particular weakness of these kinds of law, because she puts so much emphasis on the fact that "the cost of explanatory power is descriptive adequacy" (Cartwright, 1983: 3), which seems to imply that the final cost of explanatory power is the loss of the truth of fundamental laws.

In my version of the scientific process, however, that is not a problem, and, I might add, neither should it be in hers, because we both accept and acknowledge from the outset that truth is a very local and limited notion, albeit in a more complex way than is ordinarily thought. In other words, the fact that she (ibid.:5) denies that "explanation is a guide to truth", surely is only problematic if one thinks of truth as a universal notion. She does, in a sense, make amends in Nature's capacities and their measurement (1989), as well as specifically stressing pretty clearly in her article entitled Fables and mode/s (1986), the fact that questions of Irulh are not necessarily questions of universality. ${ }^{150}$

Cartwright is arguing against the notion that fundamental laws give true descriptions of real phenomena. And thus, she is also arguing against my notion of scientific progress, because although we both acknowledge the use of the notion of models to mediate between the concrete and the abstract, she still thinks that accepting some kind of realism with regard to fundamental laws means accepting an absolute notion of truth, when, paradoxically enough - as I have mentioned above - she herself still seems to believe in this notion in 
any case. Why else does she say that fundamental laws lie? If she takes seriously the possibility of contextualising the "truth" of these kinds of laws with the help of abstract models, why then does she still argue for the falsity of fundamental laws as if it is not possible for her to be satisfied with a "localised" version of truth? She argues against assumptions of the absolute truth of fundamental laws by stressing the concrete character of phenomenological laws. However, she cannot acknowledge the semantical links between theories and models that I claim exist, because she apparently thinks that would somehow imply that she believes theories to be absolutely true, and as they are not, she would rather discard them completely as part of the meaningful (and descriptive) side of the scientific process, and simply acknowledge (à la Duhem) their organising role, than try to find (like I am) some kind of reason for them to be part of the chain of factors or concepts that in the end make science mean (and explain) something to people living in the real world. (More on these issues a litlle later on in this section.)

Retuming to Cartwright's interpretation of "phenomenological", she does not use it in terms of its usual interpretation as referring to the "observable" (Cartwright, 1983; Cartwright, 1986), but rather points to the fact that this kind of law describes actual behaviour of real objects. She believes that, regardless of whether an object is observable or not, if we can manipulate it (intervene in its behaviour à la Hacking (see his Intervening and representing (1983)), we can formulate (true) low-level generalisations which accurately describe the (causal) relations into which it enters. Phenomenological laws describe particular events while whatever fundamental laws have to say is always about various situalions in reality in one sweep. So, then - because fundamental laws can supposedly do no more than explain the content of phenomenological laws (in accordance with the covering law model of explanation, about which Cartwright has quite a lot to say), and good explanations are supposed to be simple (abstract) and general - it seems that fundamental laws can indeed never directly be about any particular aspect of reality. However, it is important to understand that Cartwright, by refering to phenomenological laws as low-level generalisations, means to say that they too, have an abstract nature in the sense of being idealised descriptions of 
objects in reality. Moreover, as Chalmers (1993: 199) also points out, quite in accordance with the fact that phenomenological laws too, are generalisations that involve conditions, and even the much discussed ceteris paribus conditions, these laws themselves sometimes fail to adequately describe the behaviour of real objects. (Think, in this regard, most simply of Cartwright's example of the instructor's manual of lasers - surely lasers can malfunction?)

But how then does Cartwright conceive of relating fundamental with phenomenological laws and either (or both) of these sets with real objects? It seems that the "content ${ }^{\text {" of }}$ fundamentel lews is filled in by various abstract models. ${ }^{151}$ In Cartwright's scheme of things ${ }^{152}$, these models mediate between theories and fundamental laws on the one hand, and phenomenological laws and reality on the other. According to the model-theoretic interpretation of the process of science that I am offering, models mediate between theories (linguistic systems) and systems in reality. Phenomenological laws, in my terms, would simply be part of the content (or properties) of the models interpreting scientific theories, and they would be expressible as sentences true in the model(s) under consideration, as well as possibly true in some empirical substructure(s) of these models, and so, true of some real system. ${ }^{13}$

Schematically, Cartwright's account will look something like this:

SETS OF FUNDAMENTAL LAWS

THEORIES

MODELS

Ceteris paribus conditions active

$\downarrow$

Ceteris paribus conditions active

PHENOMENOLOGICAL LAWS

1

REALITY

My scheme of things would rather be: 
AXIOMS

FUNDAMENTAL LAWS

THEORIES

Ceteris paribus conditions active

1

CONCEPTUAL MODELS

1

EMPIRICAL SUBSTRUCTURES

1

\section{SYSTEMS IN REALITY}

Cartwright explains in How the laws... (1983: 4), that the "route from theory to reality is from theory to model, and then from model to phenomenological law", and goes on to claim that "phenomenological laws are indeed true of objects in reality - or might be; but the fundamental laws are true only of objects in the model". In other words, she does not see the same kind of referential relation between models and systems in reality that 1 see. The reason, I think, is that she worries too much about the ideal character of the models and the role of the ceteris paribus clauses needed to interpret phenomenological laws. ${ }^{154}$ Also, it is difficult to see how a very specific link with reality can be given by a law, even if it is a phenomenological one. For example, if Newton's laws are fundamental, Kepler's are phenomenological (and deducible from Newton's), but the direct observations (done in both cases) are specific ectivities (looking in a particular precise direction) carried out at a specific time (specific to the second). Statements describing these kinds of activity surely are not Jaws, but can rather be expressed in terms of some empirical model which would be a subset of the model of the theory under consideration and which interprets experimental data and empirical activities leading to the formulation of these data.

Cartwright is not a complete anti-realist, as her interpretation of phenomenological laws clearly shows ${ }^{155}$, it simply seems to be the case that she cannot see how to escape the antirealist implications of the abstract nature of fundamental laws. This anti-realism has its 
origin in her interpretation of the "explanatory" role fundamental laws play in the practice of science (physics). It seems as if Cartwright is implying - in the sense of the validity of fundamental laws being dependent on abstract, idealised situations - that fundamental laws must hold regardless of the individual arrangements of things possible in each separate situation in reality that they (these laws) are "about"; while phenomenological laws potentially describe the actual situations to which they are applied. Here again it seems as if she still holds on to some belief in truth per se ("... fundamental laws hold regardless of ... individual arrangements ..."), although her entire crusade is supposedly focussed on showing the local character of truth. Perhaps this should be taken as a warning of the danger involved in attempting to exclude the role of fundamental laws from the model-theoretic process, since it makes for certain invalid - surely unintended? - conclusions. The problem is that Cartwight does not acknowledge that the sense in which fundamental laws hold "regardless of individual arrangements" is merely in terms of their abstract nature. This should not be linked to thinking that therefore they are universally true. Nor should it be thought that because they are too general to describe real systems, they are false. Questions of truth can only be addressed in terms of the conceptual models and empirical models of scientific theories. ${ }^{150}$

In my account of the scientific process, as remarked above, I show how meaningless any talk about the truth (or validity) of fundamental laws per se is and I argue that these issues can be meaningfully addressed only in terms of the infrastructure of the models interpreting these laws. But, if then in Cartwright's terms, the main distinction between fundamental and phenomenological laws is taken to be the fact that fundamental laws hold by themselves - albeit only in certain "unreal" situations - while phenomenological laws can only hold on account of some (non-necessary) arrangement of circumstances, what does that imply for scientific explanation, prediction and the description of real objects?

Phenomenological laws describe actual events, because although they are usually mathematically formulated in physics, no fundamental explanation of the mathematical formulae nor of the mechanisms undertying these formulae are assumed in these laws. ${ }^{157}$ The 
problems related to scientific explanation in the context of the "leap" from fundamental and even phenomenological laws into more messy "worldly" situations are emphasised differently in a model-theoretic approach. Within such a model of science - as I have pointed out before - it is usually taken that scientific theories explain the content of their models, and through these models, some aspects of reality and the behaviour of certain phenomena may be described and predicted. A theory does not always necessarily explain every detail of the system in reality it is focusing on. Newton's mechanics does not explain the phenomenon of gravity. It rather explains the influence of gravity on certain events and in that sense, it describes gravity rather than explains it. The old (deductive-nomological) symmetry between explanation and description should be "stretched" such that it covers all three strata of a model-theoretic model of science. If this is not done the fact that the descriptions of gravity in the above sense may enable someone applying Newton's mechanics to make certain predictions conceming the results of the exertion of the forces of gravity, without explaining gravity itself, cannot be grasped, and then it might seem that explanatory power indeed diminishes descriptive power, as Cartwright so often claims.

Thus, in model-theoretic terms scientific theories are said to explain in the basic sense of theories explaining the content of their models by establishing deductive links between the sentences expressing what is true in some model. Thus in a model-theoretic account of science a theory and its conceptual models "explain" in the strict logical sense that a predicted phenomenon can be logically deducted from the theory and the model(s) in question. Newton's three laws of motion and his law of gravity plus the model of our solar system - in terms of current scientific knowledge - explain why we see Mars tonight at eight o'clock in a particular position. In these terms, a preceding theory (e.g. Newton's laws of motion and gravitation) may describe models which (under certain conditions, within a certain interpretation, approximately) are also models of a later "higher order" theory (say the general theory of relativity), and then the latter may be said to explain the former. ${ }^{158}$ The better explanatory power of later theories with respect to the content of their models is then the result of at least the higher level of accuracy of the theory. For instance, as Penrose 
(1997: 57) points out, Einstein's general theory of relativity can be said to be accurate to about one part in $10^{14}$, which is about ten million times as accurate as Newton's mechanics, which may roughly be taken to be accurate to about one part in $10^{7}$. Improved accuracy is one embodiment of that continuity and progress in science with which some form of realism sits comfortably.

The referential relations between model terms and objects and relations in some real system are (indeed, as Cartwright claims) more descriptive than explanatory. However, this need not result in anything as negative as Cartwright's claims of high explanatory power of fundamental laws diminishing their "truth making" power. If the whole interpretative chain i.e. from terms of some theory, to terms in some conceptual model(s) of the theory, to terms in some empirical substructure of the conceptual model in question, to some real system is taken into account, the fact that models seemingly describe and theories explain only the content of their conceptual models does not necessarily have any anti-realist consequences. Usually it is even the case that theories contain some basic notion that they merely describe, even if they do explain the rest of the content of their models - e.g. Newton's mechanics does not explain the notion of "gravity" itself, but merely describes its behaviour. The distinguishability and interconnectedness of the three stages roughly outlined by this "interpretative chain" - as set out in chapter 2 - show however that description (a feature mainly of models) and explanation (a feature mainly of theories or fundamental laws) are inseparable, perhaps even just as much as explanation and prediction have traditionally been taken to be. Just as nothing can really be said about a theory's truth or reference without linking the theory to a specific interpretation of the relevant language given by some model of the theory, explaining something means at some ("deep") level describing certain aspects of that thing. Definitions have to terminate at undefined terms, and the deduction of sentences of the theory has to start at unproven axioms.

Cartwright has a valid point in emphasising the role of phenomenological laws against the overwhelming philosophical attention that fundamental laws have been getting - and in certain cases to a certain extent, still get - but my account differs from hers, because I 
introduce the role of models from a different angle than she does. My approach, although making much of the role of fundamental laws in the scientific process, is enough in the semantic (non-statement) tradition to find her anti-realism towards fundamental laws too limiting. Unfortunately, she has few kind words to say to supporters of the semantical approach to scientific theories: "On the semantic view, theories are just collections of models; this view offers then a modem Japanese-style automated version of the covering-law account that does away even with the midwife [of deduction]" (Cartwright, Shomar, \&uarez, 1995: 139). I agree that the non-statement elimination of the theory as a linguistic expression is misguided, and that is why in my approach I stress the role of theory as much as I do the role of models. Theories (or fundamental laws) do indeed, in a certain sense, aim to "state the facts in a more general way so as to make claims about a variety of different circumstances" (Cartwright, 1983: 103). But, I see them as a crucial link in the chain of scientific progress, and I stress that it is mainly thanks to their general nature in the above sense, that they are a link in the first place.

Laymon (1989: 355) formulates one of the challenges Cartwright directs at supporters of the explanatory priority of fundamental laws as follows:

- they have to specify how actual scientific practice can be viewed as supportive of the truth of fundamental laws.

He (ibid.) points out that the first thing to do, in order to meet this challenge, is to concede that the generic-specific eccount of explanation is not descriptive and then to give it the following normative reading: "[t]he goal of science should be to seek fundamental laws which are true, and can be used in the sound derivation of phenomenological laws ${ }^{\nu}$ (Laymon, 1989; 355). He describes $(1989: 355,356)$ the realist with regard to fundamental laws as someone who believes that scientific practice can be appealed to in confirmational considerations and, in particular, that attempts to derive phenomenological laws (and data) play a role in these kinds of considerations.

Cartwright needs this challenge answered, because she wants to claim that fundamental laws explain in the sense of organising the content of phenomenological laws, but do not 
describe anything real, while phenomenological laws describe real phenomena, but do not really explain them. ${ }^{159}$

Realists (in Laymon's terms) explain the practice of looking for increasingly more accurate and less idealised initial or boundary conditions in terms of the fact that idealisations are characteristically false and that they therefore have a distorting influence on derivations of predictions in such a way that, even if the fundamental laws are true, they will be able to produce onfy distorted or false predictions. In this sense, Laymon (1989: 359) claims, realists see the aim of science as the construction of more accurate models because they believe that our theories, if true, will produce more and more accurate predictions when applied to these more and more accurate models. He (Laymon, 1989: 359) gives the example of Baily's connection of coefficients rendered superfluous and corrected by Stokes's development of a Newtonian theory of viscous fluids to illustrate his point. Cartwright (1983) gives a few examples in quantum physics to illustrate this realist tendency about approximation among "fundamentalists" - most notably Messiah's hydrogen atom (Cartwright, 1983: 137-138), and Louisell's treatment of the gas laser (Cartwright, 1983: 146 - 148).

Well, is giving idealised (perhaps "approximately true") descriptions (or explanations) of real systems not at least one of the things that science "really" is about? The trick is perhaps to distinguish between "distorling" idealisation and counterfactual idealisation. Think again of Suppe's distinction between counterfactual and ideal truth. Models provide us with counterfactual truths in the sense that they realise cerlain selected parameters of the theory in specific contexts where certain other influences are held stable. However the physical systems thus described are causally possible, while the kind of distortion to which Laymon and especially Cartwright seem to be referring, is Suppe's notion of "pure abstractions" that is not part of the empinical truth of models. (See Chapter 3 for a discussion of Suppe's notion of empinical truth.)

In the light of these examples Laymon (1989: 359, 360) formulates the following confirmation principle:

- a set of fundamental laws receives confirmation if the use of more realistic specifications 
of initial or boundary conditions in fact leads to more accurate predictions.

He concludes from that that sets of fundamental laws (i.e. "acceptable" theories) are "monotonic towards the truth" (Laymon, 1989: 360) in the sense that more accurate and less idealised initial conditions lead to more accurate predictions. This implies that, from theory $T$ being monotonic in certain situations, we conclude that $T$ is, in principle, monotonic, that is in other words, that $T$ is such that if $T$ is true, then it is possible, in principle, to make sufficient corrections to its initial conditions so that it will yield better predictions. Laymon (1989: 360,361 ) claims that this monotonicity approach offers a way in which to show how fundamental laws can be confirmed or disconfirmed in the face of distorting idealisations, although he concedes (1989: 357 ) that a fundamental realist asks too much if she wants to be able to claim that it is possible to derive phenomenological laws and also, more generally, empirical data from some true fundamental law, or a set of fundamental laws. (He (1989: $361,362)$ cites the Michelson-Morley example and a few others to illustrate this.)

The model-theoretic interpretation of science that 1 offer accommodates both the possibility of this kind of development (in the sense of constituting models to interpret the fundamental laws) as well as fundamental laws being monotonic towards the truth - think of the events leading to the discovery of Neptune. It was possible to amend the model of the solar system with which scientists had been working up to the discovery of Neptune, without implying that the older model was necessanily "false" (in the sense of not referring to anything in reality anymore - if it ever did at all). This is also related to the fact that I take the problem of underdetermination to be twofold: theones (and intended models) are underdetermined by data - the old Duhem-Quine problem; and models are underdetermined by theories - the set of sentences of a particular language which are true in a particular model of a theory is bigger than the set of sentences comprising the theory. This implies that if a theory is underdetermined by data, a model of that theory is a fortion underdetermined by those data. See Chapter 5 for a discussion of underdetermination in model-theoretic terms.

Laymon (1989: 363) accuses Cartwright of ignoring the "confirmational value" of this sort of improvability of idealisation and approximation which is an actual fact of real scientific 
practice, although he acknowledges that he needs inductive evidence for his claim that this kind of improvability leads to stating that, if a set of fundamental laws is true, then it is possible, in principle, to make sufficient corrections to its initial statements (conditions) so that it will yield better predictions. He discusses (1989: $366-368$ ) in this context, the example Cartwright (1983: 107ff) gives of Bethe's derivation of the Lamb-shift (in the excited state) by correcting the Weisskopf-Wigner method of treating exponential decay, and also Agarwal's derivation of the Lamb-shift in the ground state. Laymon concludes (1989: 367) that all that can be said in the light of these examples is that some combination of approximations and idealisations entail the Lamb-shift - whether or not quantum mechanics entails the Lambshift depends on the approximations and idealisations scientists decide to use, and on nothing more. In other words, Cartwright's anti-realist argument in this regard is based on two factors:

- the choice of approximations and idealisations is limited by the initial and boundary conditions which have to be added to fundamental laws (via their conceptual models), but not dictated by them; and

- different choices lead to different incompatible results.

As shall be pointed out in Chapter 5, my treatment of the underdetermination problem shows that these factors need not have any anti-realist implications at all. For instance, the fact that initial and boundary conditions limit the choice of models, but cannot dictate scientists' choices - the first factor above - is completely accommodated within the framework for interpreting the progress of science that 1 am offering, and actually contributes to the continuous character of science.

These factors lead Cartwright however to conclude (Cartwright, 1983: 107) that the empirical content of the phenomenological laws is not contained in the fundamental laws which supposedly explain them, in other words, she concludes that the fundamental laws simply organise scientific knowledge by explaining phenomenological laws but cannot really describe - or say anything else for that matter about - the real objects in the real world. The main reasons for Cartwright's antirealism about fundamental laws can be summarised as 
follows:

- She believes that the fundamental laws of science (physics) do not describe the behaviour of the objects in their domain, rather they "provide mathematical frameworks into which, by various devices some phenomena of the world can be fitted" (Chalmers, 1987: 84). In other words, to give a fundamental theoretical account of an object is to "fit" that object into the mathematical framework of the theory (for examples see Chalmers, 1987: 84, 85) - which means that the laws explain idealised versions of the behaviour of real phenomena by some underlying (usually mathematical) mechanism.

- She takes the underdetermination of theory by data as proof for the anti-realist nature of fundamental laws (she offers the example of radiative damping as an example, see Chalmers's discussion of this example in Chalmers, 1987: 86). She claims to successfully address the underdetermination problem however by showing the real nature of capacities in her later works. (1 "solve" the "problem" of underdetermination by simply incorporating it into the structure of the scientific process.)

- She emphasises the logical gap between fundamental theoretical descriptions and adequate phenomenological descriptions of real situations by pointing to the imprecisions in mathematical expressions of theoretical descriptions (she offers the quantum mechanical treatment of the Lamb-shift as supporting evidence for this claim - see a discussion of this example in Chalmers, 1987: 86) - instead of stressing the interpretable nature of these kinds of expressions and examining the possibility of them having "links" with reality via models.

Chalmers (1987: 87) writes: "Cartwright takes on an anti-realist stance with regard to fundamental laws, then, because the situations described by them are too simple and artificial to correspond to real world situations [no description of real objects], because adequate descriptions of the latter cannot in general be logically deduced from fundamental laws in conjunction with initial conditions [against the covering-law model], and because physicists frequently employ fundamental laws in diverse ways to offer different descriptions of the one real world situation [underdetermination of theory by data]". 
Laymon (1989: 367) counters that one simply has to accept that judgements of "relative realism" are often made before the accuracy of predictions are compared. He claims that for instance, with regard to Bethe's derivation of the Lamb-shift, "Bethe's realisation that the mathematical debris [-] generated when the time approximation was applied before the sum approximation [-] could be interpreted as the Lamb-shift, may have served as a good reason to grant his calculation the status of being more realistic than the onginal Weisskopf-Wigner calculations" (Laymon, 1989: 368). In other words experimentation can - and is actually used to test the relative realism of idealisations. Which is essentially what I am claiming too. The idealised nature of models necessitates a variety of different empirical (mostly experimental) ways to select different interpretations (models) of one theory, namely those models which are able to accommodate the empinical models generated by the data. This, however, goes against Laymon's own confirmation principle (Laymon, 1989: 359, 360 and aboye) because these examples offer no illustration of the use of the previously determined relative realism of idealisations to test furidamental theories.

\subsection{The role of models in science and Cartwright's "simulacrum" account of science}

Traditionally, according to the statement approach, in philosophy of science a theory is taken as consistirg of two parts:

- internal principles (the "core" or basic content of the theory expressed in some theoretical language), and

- bridge principles (links or procedures "giving meaning" to the theoretical terms in the theory's language by relating these terms to phenomena in the "external world").

A problem that has been worrying Cartwright - and which occupies any philosopher concerned with dealing with the intricacies of realism, maybe especially those working from a model-theoretic point of view - is, very simply put, that the presence or necessity of these "bridge principles" of an indirect and complex nature, however they are interpreted (in terms of models, mathematical functions, both, or something entirely different), implies somehow that the theory ${ }^{100}$ itself has very little to say about the real phenomena the bridge principles 
are supposed to link it to.

First on her mind in How the laws of physics lie (1983, chapter 8$)$, is to make clear whether having as few as possible bridge principles, should hold a promise of high explanatory power. I suppose the reasoning behind this claim is that the fewer bridge principles a theory needs the less "fundamental" - in the sense of falsity - its fundamental laws. She (1983: 143) claims that, if members of some research community want to be able to work together, some way has to be found in which to limit the kinds of models that can be used to describe real phenomena, because, given the complex and rich nature of these objects (the phenomena that have to be described) a variety of models might describe the same phenomena. She (Cartwright, 1983: 144) writes :

If there were endlessly many possible ways for a particular research community to hook up phenomena with intellectual constructions, model building would be entirely chaotic, and there would be no consensus of shared problems on which to work. The limitation on bridge principles provides a consensus within which to formulate theoretical explanations and allows for relatively few free parameters in the construction of models ... It is precisely the existence of relatively few bridge principles that makes possible the construction, evaluation, and elimination of models. This fact appears also to have highly anti-realist side effects ... it strongly increases the likelihood that there will be literally incompatible models that all fit the facts so far as the bridge principles can discriminate ... ${ }^{161}$ The great explanatory power of quantum mechanics comes from its ability to deploy a small number of well-understood Hamiltonians to cover a broad range of cases, and not from its ability to match each situation one-to-one with a new mathematical representation.

The anti-realist implication of this is, I agree with her (Cartwright, 1983:145), not to be argued against by some belief in only a very small number of basic interactions in nature, but rather, I claim, by a belief in the very nature of the link between models of a theory and some aspect of reality. I do not think though that these links are determined solely by traditional bridge 
principles, because the notion of bridge principles too has too much of a universal air about it. I am more comfortable with the more "natural" limitation on models provided by the aims, background information, equipment, and training of the specific scientific community in question, as well as the satisfaction functions operating between theories and their models that set out the specific boundaries of the content of models in the first place. I am not entirely convinced though, that Cartwright would completely agree with that, given her anti-realist interpretation of the question of underdetermination, as well as her repeated efforts to show that terms in a theory have nothing to say about any aspect of reality, but can only explain the idealised behaviour of objects in models of the theory, thus effectively still severing the realist link.

She (1983:147 - 150) does however distinguish two senses of the notion of the "realistic" nature of a model:

- The first sense has to do with the relation between a model and reality (some aspect of reality, I would say). In this sense, a model is realistic if it gives an accurate description ("picture") of the aspect of reality ("situation") being modelled. In other words it will have to describe the structure and actual behaviour of the real system.

- The second sense has to do with the relation between the model and "the mathematics" (in her terms (1983: 150)), which is the relation between the model and the theory in my terms. According to her a fundamental theory determines criteria for what counts as explanations, and, in these terms - relative to those criteria - a model will be realistic if it explains the mathematical representation - i.e. if it realises the theory. ${ }^{162}$ She (Cartwright, 1983: 151ff., chapter 8) offers her "simulacrum account of explanation" in the place of the covering law model of explanation. According to her (Cartwright, 1983: 151) the covering law model requires the way in which phenomena are modelled to be realistic in both senses because it views a phenomenon to be explained if it has been derived from some fundamental law. Cartwright, however, primarily wants to show that - and how fundamental laws logically summarise and classify (as mentioned before, in Duhem's tradition) groups of phenomenological (experimental) laws without aiming to explain them. 
She (ibid.: 152) writes: "I have been arguing ... that the vast majority of successful treatments [of phenomena] in physics are not realistic. They are not realistic in the first sense of picturing phenomena in an accurate way; and even in the second sense, too much realism may be a stop to explanatory power, since the use of 'phenomenological' [still abstract] terms rather than more detailed 'causal' constructions may allow us more readily to deploy known solutions with understood characteristies and thereby to extend the scope of our theory [although this will not necessarily lead to a better understanding of the actual aspect of reality the fundamental laws are 'about']'.

Cartwright's problem with fundamental laws is that they are laws about distinct (separate) aspects of objects in reality and their behaviour - or, in her most recent terms, about distinct causes and their separate effects - while, in the real world, these things actually occur only in combination with other aspects of these or even other objects. And, moreover, these combinations change quite often and occur very seldom according to some regular kind of pattem, because of the variety of factors involved. Cartwright's problem is that "[e]ven if these regularities did hold ceteris paribus - or, other things being equal - that would have no bearing on the far more cornmon case where other things are not equal" (Cartwright, 1989: 177). Again, this interpretation of the nature of fundamental laws is not really what is at issue here, the problem or challenge really is to find a kind of view that can accommodate these fundamental features of scientific theories and still offer a realist interpretation of the scientific process. A model-theoretic approach such as the one that I am offering holds this promise without even having to specify whether one sees objects and activities in reality in terms of causes and their separate effects (as Cartwright seems to be doing nowadays) or not, since both accounts can be accommodated.

Now, if giving a fundamental theoretical account of a certain object means fitting it into the mathematical framework of the theory under discussion, and, if this is what fundamental laws ultimately do, as Cartwright claims - "To explain a phenomenon is to find a model that fits it into the basic framework of the theory and that thus allows us to derive analogues for the messy and complicated phenomenological laws that are true of it" (Cartwright, 1983: 152) 
- the obvious question to me is why this should result in false fundamental laws? The answer lies in Cartwright's notion of models and their role in the scientific process.

She (ibid.) points out that models help us to "see" the relevant phenomenon through the mathematical framework of the theory, but stresses that different problems will have different emphases on different aspects of that framework. This, to me, implies that different models can - and should - only be evaluated according to the different aims guiding their construction. And that is, in my view, why she calls her account of explanation a "simulacrum" account. ${ }^{163}$ She (ibid.) writes:

This is just what I have been urging that models in physics are like ... A model is a work of fiction. Some properties ascribed to objects in the model will be genuine properties of the objects modelled, but others will be merely properties of convenience ... Not all properties of convenience will be real ones. There are the obvious idealisations of physics - infinite potentials, zero time correlations, perfectly rigid rods, and frictionless planes. But it would be a mistake to think entirely in terms of idealisations - of properties which we conceive as limiting cases, to which we can approach closer and closer in reality. For some properties are not even approached in reality. They are pure fictions. I would want to argue that the probability distributions of classical statistical mechanics are an example ... It is better, I think, to see these distributions as fictions, fictions that have a powerful organising role in any case and that will not mislead us too much even should we take them to be real in the simple cases. ${ }^{164}$

However, "[b]eing explanatory in this sense, that is, being useful in many different contexts, requires the theory to neglect the special differences between the contexts ...Therefore, the theory cannot be true of any of these real situations; it can give a correct description only of the behaviour of objects in highly idealised contexts or models. The model contains the distortions and idealisations that are necessary to make a theory bear on a real situation. Real objects and their behaviour are too varied, too complex, too messy to be treated faithfully by theories of great generality; that's why we need models to mediate between 
theory and phenomenon" (Rueger \& Sharp, 1996: 95). The important thing that both Rueger and Sharp, and also Cartwright, seem to overtook is that scientists never examine any real system in all its messiness. That simply is not - and has never been - the aim of science. It is however part of the task of philosophy of science to show how such abstract and general theories may be said to be (or not to be) about aspects of this complex reality, and yes, that is where studies of the internal structure of models of theories and the various relations into which they enter come in. The mediation of models between theory and reality is however misunderstood if it is taken to offer the means by which science can indeed be said to say something about some real object in all its varied complexity.

And, it seems that (for Cartwright) it is because of the simulacrum nature of models that bridging relations can only hold ceteris paribus. This shows the structural error in her account as far as ceteris paribus conditions are concerned. The view that portrays these conditions as some kind of ingenious device cunningly designed by naive realists or staunch fundamentalists to "save theories from point-to-point testing" (Rueger \& Sharp, 1996: 103) ${ }^{165}$ is completely misguided. First, however, as far as bridge principles are concemed - they do not hold ceteris paribus. There is no absolute set of rules describing these kinds of correspondence relation. Rather these rules hold with respect to a certain model within whose boundaries the theory is true. The best that can be said about "bridge principles" in my terms is that perhaps one can speak of a set of bridging "procedures" or "links" that extracts data from the relevant real system relative to a specific empirical context, and then injects these data, as an empirical model into the model under consideration.

The theory holds ceteris paribus yes, but not in Cartwright's sense of the word. In my terms, to say that a theory "holds" means, per definition, that it holds (is "true") in a particular one of its models. To say now that it holds "ceteris paribus" adds nothing to simply saying that it is true. Moreover, there is nothing else about which it can be stipulated that it stays the same - everything is given in the model. Ceteris paribus clauses seem in Cartwright's terms to play a more and more important role the further away one moves from fundamental laws. In model-theoretic terms, however, they are necessary only at the level of scientific theories 
or linguistic systems, and become less and less active the closer to reality one moves. I claim - see also Chapter 2 - that they are suspended in their generality as soon as the theory in question is interpreted in specific models, rather than activated. The idealised nature of conceptual - and even empirical models - is not the result of specific ceteris paribus clauses, but indeed simply true to the nature of science. No real system can ever be examined, represented, explained, or described in its full complexity. That is simply not science's function.

Following Duhem (Duhem, 1914: 7), Cartwright (1983: 96) considers the notion of scientific explanation in terms of description in the sense that explaining a set of phenomena means giving a physical theory of them, "a physical theory in Duhem's sense, one that summarises ... and logically classifies them" (Cartwright, 1983: 96). Rueger and Sharp (1996: 95) refer to the problem she has with the covering law account of explanation as the "unsoundness argument" and set it out as follows: "If ... phenomenological laws could be soundly derived from more fundamental laws as the traditional [covering law] view would have it, then any successtul comparison of the phenomenological consequences of the theory with the observations would count unproblematically as inductive support for the theory. Confirmation would flow upwards from the phenomenological level to the fundamental level. This flow, however, is staunched ... because phenomenological laws typically cannot be soundly deduced from more fundamental theories. To denive the former we usually need assumptions [ceteris paribus clauses] which are either false (distorted representations of the situation of application) or which contradict the fundamental laws themselves. Inductive support cannot, therefore, be transmitted."

Claiming that phenomenological laws cannot "typically" be deduced from fundamental ones, is perhaps jumping the gun a bit. Is it not the case that Kepler's laws can be deduced from Newton's in a very sound way? Moreover the cerenis paribus clauses and other additional assumptions needed to validate the fundamental laws are suspended when models are constructed of some theory - as remarked above - and thus these clauses become more and more concretely realised as they set the boundaries for the truth of the theory, i.e. 
the clauses themselves (e.g. "no other forces act differentially on components of the system") become realised, i.e. true in the relevant models. Thus it is rather unclear how they can be understood to "contradict" the fundamental laws themselves (which are also true in these models). ${ }^{168}$ These ceteris paribus conditions or clauses will usually be incorporated into the formulation of the law explicitly (as when stating that Hooke's law holds as long as the elastic limit has not been exceeded), or else implicitly and tacitly by common understanding.

So, it seems that to Cartwright, in order to fit some phenomenon into the mathematical framework of some theory, a model of that phenomenon "which re-describes it in terms which are amendable to mathematical theoretical treatment" (Chalmers, 1993: 200) has to be constructed. "Before we can apply the abstract concepts of basic theory - assign a quantum field, a tensor, a Hamiltonian, ... or write down a force function - we must first produce a model of the situation in terms the theory can handle" (Cartwright, 1994b: 282). I do agree with this, but not with Cartwright's conclusion that models are thus (in her terms) merely devices used to fit phenomena into the theoretical frameworks of theories which in their turn classify and organise groups of phenomena. - "... the point of the kind of models I'm interested in is to bring the phenomenon under the equations of the theory" (Cartwright, 1983: 157). This is nat the only role models have. They do have this function, but it is by virtue of this very function that they offer ways to link the theory with aspects of reality.

Cartwright (1983: 160) wants to focus on what "actually happens in concrete situations, whether these situations involve theoretical entities or not, and how these differ from what would happen if even the best of our fundamental laws played out their consequences rigorously. Moreover, she stresses (1994b: 292) that Hacking's point in Representing and intervening (Hacking, 1983) is not merely that theoretical entities exist if we can use or manipulate them or intervene in their behaviour, but far more important, that "... it must be the case that we understand their behaviour very well if we are able to get them to do what we want".

She (1994b: 292) concludes then from this that such an understanding should be taken as enough evidence for "... the truth of some very concrete, context-constrained claims, the 
claims we use to describe their [the theoretical entities' under discussion] behaviour and control them". The important fact here remains that (ibid.) "... in all these cases of precise control, we build our circumstances to fit our models ... [in other words] that does not show that it must be possible to tailor our models to fit every circumstance. ... some circumstances resemble the models we have; other do not. And it is just the point of scientific activity to build models that get in, under cover of the laws in question, all and only those circumstances that the laws govem [try to describe]". She already says as much in How the laws of physics lie (1983: 157), where she clearly states yet again that she is arguing, not that the generality and exceptionlessness of fundamental laws are proof of their being laws of nature, but precisely the opposite, namely that these features are not real, that is, fundamental theories simply appear to have these characteristics. This is the result of an over-focussing on what she calls the "second stage of theory entry", in the sense that the fundamental laws may be true of the objects in the model (but not of objects in reality), "but that is because the models are constructed that way ... when we present a model of a phenomenon, we prepare the description of the phenomenon in just the right way to make a law apply to it (ibid.).

I suppose we do, in a sense, try to "make" the circumstances resemble the models we have, at the stage when the intended model is being constructed, insofar as the aims of the scientists concerned, the available mathematics, and so on, will define the nature of the models at that level. Also, perhaps at the stage of the scientific process where empinical adequacy is tested these kinds of considerations will have some role to play. In a sense, one's view on this will depend on the role one takes background information to play in the process of science - are scientists trying to find a "match" between some model and an aspect of reality, or an aspect of reality-as-they-interpret-it according to their established body of knowledge, future research aims, and training? More about this a bit later on in this chapter. A last remark on saving the phenomena: the construction of models means precisely the preparation of the conceptual description of a certain object in reality to "make" some law apply. However, no a prion rules exist - as pointed out above in terms of bridge principles - that govern the construction of models, and therefore, obviously scientists cannot 
guaranlee that these deliberate actions will indeed end in descriptions of real phenomena. I am not sure though whether philosophy of science should demand such guarantees. I think that being able to describe in general the way in which theories are linked to aspects of reality, and so to set out the way in which (the process of) science should be interpreted, should be enough. The guarantees at issue here are at the level where relations betweon aspects of reality and modeis are evaluated, and that is solely an empinical issue, and thus falls in the domain of science itself.

This also has to do, as Cartwight (1983: 158) points out, with the anti-realist implications of her account, because different - possibly incompatible - models are used for different purposes, and so there is no one-to-one matching between models and the real situations being studied. And, obviously then, that is why she cannot see how the laws goveming the models could be presumed to apply to real situations. According to Rueger and Sharp (1996: 107) fundamental theories cannot receive confirmation from successful predictions, based on models of theories, precisely because these models themselves are no mirror-images of reality but rather distort reality - because of their ideal (or context-dependent or) aimorientated emphasis-sensitive nature. Then one may well ask how, if there is no one-to-one mapping between a situation studied and a model, and a fundamental theory is true only in a model, can it be claimed that a scientific theory has something - or anything for that matter — to say about reality? Of course, this makes sense, but I am claiming that these issues can be viewed and set out a little differently:

- It is true that there is no guarantee that some theory will be applicable to a specific aspect of reality before that theory has been interpreted and this interpretation (model) linked empinically to some system in reality.

- But, what does it mean to "place a phenomenon in the mathematical framework of a theory"? Does that not already imply that the model was constructed — apart from giving a true interpretation of the theory - also with some kind of real phenomenon in mind? Yes, indeed.

In other words, the model-theoretic view of science implies that scientific theories can - and 
do - say something about reality (because of the way the reality-model-theory link is interpreted), but acknowledges that it is not possible beforehand to determine or claim that a certain theory will definitely be applicable to a certain aspect of reality and to no other. The reasons for this are very important since they explain the "vagueness" of any kind of description of "bridge procedures" between theories, models, and real systems. The first reason lies in the "freedom" involved in the construction of models in the sense of model construction being influenced, among other things, by the scientific community's aims with the theory under discussion. Entangled with this reason, the second reason lies in the specific nature of the real system that the theory in the end - via its conceptual and empinical models - may be said to be "about".

Cartwright does see this, in a way, since she (Carwnight, 1994c: 293) acknowledges that the link between (an aspect of) reality and some model is "a matter for hard scientific investigation, not a priori metaphysics". And goes on (ibid.) to explain that "[t]hat is the reason I am so concemed with the successes and failures of basic science in treating large varieties of situations differing as much as possible from our experimental arrangements". This is a valid point, but only worrying if one believes that science is mirroring total Nature by discovering its ultimate laws. Otherwise, this simply is a feature of the way science is being done.

So, although models are the source of the "distortions" and idealisations that prohibit theories (and their fundamental laws) to say anything directly about any real situation, modeltheorists like me cannot see the scientific process continuing without them. But, that simply brings us back to Cartwright's claim that laws that explain are not necessanily true. Rueger and Sharp again (1996: 96): "There is thus a trade-off between a theory's explanatory power and its (potential) truth: the more efficient a theory is in explaining or organising a large variety of different phenomena, the less can it be true or state the facts". As Cartwright (1983: 72,73 ) herself has been stressing since How the laws of physics lie (1983),

[i]f we state the fundamental laws as laws about what happens when only a single cause is at work, then we can suppose the law to provide a true description. The 
problem arises when we try to take the law and use it to explain the very different things which happen when several causes are at work. This is the point of 'The truth doesn't explain much'. There is no difficulty in writing down laws which we suppose to be true: 'if there are no charges, no nuclear forces, ... then the force between two masses of size $m$ and $m^{\prime}$ separated by distance $r$ is $\mathrm{Gmm} 1 r^{2}$. We count this law true - what it says will happen, does happen - or at least happens to within a good approximation. But this law does not explain much. It is irrelevant to cases where there are electric and nuclear forces at work.

Well, yes, of course, if one believes in an absolute notion of truth, and if one believes that this absolute truth in science is about specific individual situations in their uniqueness. That is the whole point! It is, I believe however, possible to speak only of theories being truein-some-model and not of theories being true qua nothing else, i.e. absolutely or "universally" true. Cartwright (1986) does point out the fact that "truth" does not mean "universal", but almost everywhere else she continues to contradict herself. I think the reason for these ambiguities may lie in her interpretation of ceteris paribus clauses or conditions. She views these clauses as playing an important role in the explanatory power of the fundamental laws, in the sense that they determine what kinds of explanation are permissible because they lay down or record, in a sense, the nature of the abstractions from real situations made by the theory and its fundamental laws. It seems then that in this sense the conditions laid down by these clauses also determine the nature of the models of the theory in question in their function as part of the concretising mechanisms of science, such that they "adapt" contexts to "fit" the laws explaining the behaviour of the objects found within that particular context.

In model-theoretic terms the role - if any - of ceteris paribus clauses is somewhat different - as I have already pointed out. First, they are only of importance - if at all - as part of the linguistic expression of some theory. Cartwright's reconstruction of Newton's gravitational law (see above quote) - i.e. "If there are no ..." is simply wrong. The law states rather that "The gravitational force between two masses ..." without exception. The law is still absolutely and totally relevant when there are (also) other forces present! What she implies 
with her reconstruction of the law is simply not the case. The gravitational force is still there in cases where electric and nuclear forces are at work. We take the (vector) sum of all the forces on a particular body to see how it will behave. I might also remark here that thus, a remark such as Rueger and Sharp's (1996: 96) conclusion concerning Cartwright's thoughts on this issue: "Because the complexity of the behaviour of real objects is produced by the interaction of hopelessly many (causal) factors, varying from context to context, a simple, highly explanatory theory which inevitably ignores most of these factors has (almost a priori) no chance of ever providing a true description of a [real] situation", is equally wrong. We do have theories and models about how different causal factors combine when acting on the same system in reality. A simple example is given by the vector addition of speeds, accelerations, and forces. The only kind of reconstruction of Newton's gravitational law that mentions other forces and factors should then simply be something like this: "Even if there are electric charges, nuclear forces, the sun shining on them, rain falling on them, ..., then still, everywhere, under all possible cincumstances; the gravitational force between two masses..."

Ceteris panibus clauses, where necessary, form part of (a complete formulation of the law and are not even really extraneous conditions. A scientific theory makes statements concerning the nature and behaviour of a certain phenomenon, ar a group of phenomena, in some real system(s). These statements are "sweeping" precisely because they have to cover all phenomena, in whatever context they may occur, that may exhibit the features of the ones described in the theory, and not only specific ones, In that sense, the formulation and application of any fundamental law is never ceteris paribus. Rather than saying "if all possible influencing factors not explicitly mentioned are absent or neutralised, then ...", a fundamental law will typically say "even if all possible other factors influence the system in all possible ways, then still .... Of course, the complete formulations of many (fundamental and phenomenological) laws are conditional. Remember again Hooke's law: "If the elastic limit has not been exceeded, then ....".

The specific clauses that Cartwright has in mind, are thus much rather part of the 
content of the (conditional) law expressed by the theory in question, than conditions for the law's applicability. Ironically enough, if these clauses are conditions (in Cartwright's or in the logical sense) it implies that they can be negated - l.e. not be satisfied - and the conditional formulation of the law can still apply. (It is a case of simple logical equivalence that $c p \rightarrow$ law $\Leftrightarrow \neg c p \vee$ law). One reason why Cartwright sees ceteris paribus conditions as separate from the law and will probably not accept my incorporation of them into a conditional formulation of the law, is the following. Her ceteris panibus conditions may involve influencing factors (for the physical system under consideration) for which there are not even terms in the language of the theory. Look again at her reconstruction of Newton's law of gravity: it drags in ceteris paribus conditions involving electricity and nuclear forces, about which the language of Newton's theory cannot even talk. So it is impossible to formulate her reconstruction of the law as a conditional sentence in the language of Newton's theory. What we have here is another manifestation of Cartwright's aim (which is again, not the aim of science) to scientifically and truthfully encompass a system in all its limitless complexity and interrelatedness. Maybe her "patchwork" picture of the world (see Section 4.6) is her loophole to investigate what would otherwise be inexorably entailed by her view, namely that there is only one possible object for science: the whole cosmos.

The conceptual models of theories determine the idealised context(s) - i.e. (truth) conditions - within which the theory will be true, simply because that is what an interpretation of a linguistic expression does. The idealised nature of models is not "ideal" by virtue of any ceteris paribus conditions added to the theory. As I have stated before, ceteris paribus clauses are suspended (in their generality) when the theory is interpreted in its model(s) - in the same way in which the law's generality is suspended, as is now evident form the above. Perhaps Cartwright misinterprets the reason for the fact that truth and universality are different concepts. She (Cartwright, 1997: 167) claims that "To say the laws of physics are true ceteris paribus, is not to deny that they are true. They are just not entirely sovereign". Well, model-theoretically, theories are indeed not simply true, whether conditional, ceteris paribus, or not, but they are sovereign. Theories can only be true in their models, 
regardless of how many - if any - ceteris paribus clauses form part of their formulation. Moreover it is exactly because they are sovereign, in the sense of being formulated for all possible circumstances satisfying their terms, that they have any chance at all to be true (in their models).

Now, let us look again at Cartwright's distinction between universality and truth. She (Cartwright, 1989: 162) claims that theories should not be taken as summaries of laws about observable entities, because theoretical entities are not needed to explain the behaviour of observable entities, but are rather necessary to systematise observable behaviour - as pointed out before. Very much in the constructivist tradition, she then goes on to stress that theories are never universally applicable, but their domain (the limit of their applicability) is determined by making use of the theory and its concepts themselves. This is interesting in the sense that, as far as the constructivists are concerned, it makes it impossible to ever move to a meta-level for any reason - like evaluating the scientific content of a theory. What Cartwright wants to show, I think, is also along these lines, although she is, more specifically, aiming to show that truth and universality do not necessarily imply each other. This, of course, is entirely in line with a model-theoretic interpretation of these notions. The notion of universality, in model-theoretic terms, is not applicable when it comes to science, and the notion of truth, though still important, is ultimately based on the notion of context-dependent empirical adequacy.

All of this illustrates the necessity of the interpretative role models play in science. In an article entitled The tool-box of nature (1995), that she co-authored with T.Shomar and M.Suárez, Cartwright again claims that "[r]epresentations of phenomena must be constructed and theory is one of the many tools we use ..."(Cartwright, Shomar, Suárez, 1995: 139). (My italics.) She goes on: "I want to urge that fundamental theory represents nothing and there is nothing for it to represent. There are only real things and the real ways they behave. And these are represented by models, models constructed with the aid of all the knowledge and technique and tricks and devices we have" (ibid.: 140), I have no quarrel with these remarks. That is exactly what 1 am trying to show, in the sense that I want to establish the 
fundamentally "constructed" nature of science. But, although in a sense I urge the "constructedness" of theories just as much as the "constructedness" of models, I view the role of theories and their abstract and general nature as a central part of what science is, while Cartwright more often than not sounds as if she would much rather do without theories, although she of course does acknowledge their organising features. And the reason for that is, I think, that she focuses too much on the spurious theory-reality link at the cost of the construction of the theory-model-reality link.

I see the role of theories within the whole representation process as more meaningful - and maybe more useful - than she sometimes seems to do. To me models are constructs, that is (conceptual, i.e. mathematical) structures that do not have to be primarily linguistic, while theories are primarily linguistic entities (sets of sentences of some appropriate language, that - among other things - describe models in which those sentences are true). In my view therefore, theories are absolutely essential to science, because they formulate the conceptual content of the models, make this content amenable to deduction and computation, and communicate this content to other scientists. The main means of communication in science still is language (together with diagrams, physical models, demonstrations, films, and so on).

However, the arguments Cartwright sets out against the "theory-driven" (Cartwright, Shomar, Suárez, 1995) approach to models fit well into my model-theoretic account of science. It is true that models are not simply deductions from theories, nor is it possible for any kind of nomological (law-like, universal, always-the-same) link between theories, models, and reality to exist. Briefly the "theory-driven" approach has to do mostly with that old realist favourite: approximation. However, this kind of account of science and its theories (in terms of approximations) seems somehow to imply a kind of apriori-ness about models, in the sense that each new (approximating) model will always definitely bring us closer to "the truth" than the previous one. I claim that in general no such guarantees can be given, and I think that Cartwright would agree. The advocates of the theory-driven view - according to her (ibid: 148 ) - see the construction of a new approximating model as one of the following 
activities:

- certain correction factors - that are strongly motivated from the perspective of physical theory - are introduced into the theory's equations;

- or, some other model is found for the (revised) theory which implies the revision of some of the standing physical assumptions. ${ }^{107}$

Cartwright and company (ibid.) are advocating a much more free notion of models in the sense that (phenomenological) model construction should be viewed as far more independent — in method and aim - from the theory in question. In a sense, at least as far as the limited power of the formulators of the original theory over its resulting logically possible interpretative models is concerned, it might seem as if that is what I am advocating too. The semantic link between theory and model in my approach, however, has to remain. Establishing this link is simply not applicable or relevant in a non-statement framework, given their disregard of scientific theories as linguistic entities (although they of course do not deny that models are interpretations in which a given theory is true).

Anti-realist supporters of a semantic view of the scientific process, especially someone like Bas Van Fraassen, aim to say as little as possible about the "raw" data used as a starting point of the process of science (Van Fraassen rejects all empiricist theories of meaning), and simply try to fit these data "into some single consistent model [an empirical substructure in Van Fraassen's case] which exemplifies the laws and theories as well" (Cartwright, 1989: 168). Cartwright (bid.) criticises Van Fraassen however for not explaining how exactly the raw data are supposed to be fitted into some model or theory. It is true that he only discusses cases where low-level theories (her phenomenological laws I think) are fitted into models of high-level theories (her fundamental laws), but never says anything about the lower level fitting of raw data to phenomenological laws or - in my terms - into some empirical model of the (fundamental) theory. Cartwright $(1989 ; 168)$ does acknowledge that he might have felt that this second kind of fit is aiready implied in the process of justifying or confirming the theory. As I have pointed out in the explication of my model-theoretic account in the previous chapters it is extremely difficult - even impossible - to formulate a set of "correspondence 
rules" to dictate the nature of the final realist link form model to (system in) reality. Speaking of empirical adequacy - both in Van Fraassen's narrow and my broader definitions of it is all that can be said in general. The actual factors at work at that level of science are too model - and (real) system specific to allow closer or stricler definition - in an absolute sense — of what happens so that a theory may "finally be announced 'empirically adequate".

Cartwright (1989: 168 - 169) stresses that, in any case, interaction with raw data is impossible in the following sense: "Scientific claims are tested, not against the empinicist's data (e.g. 'individual temperature readings from samples of lead, bubble chamber photographs') but rather against 'phenomena detected from them (e.g. the melting point of lead, the weak neutral current, changes in the rate of solar neutnino emission')". This points to the "dual" nature of models (in my terms) in the scientific process: not only do models interpret the abstract theories, but conceptual models via their empirical submodels also "help" with theory-formulation as mediators between nich complex aspects of reality and the research aims (and other context-dependent factors) influencing a given scientific community.

Also, Cartwright $(1989 ; 169)$ claims that the questions concerned with dealing with raw data or not, involve the whole ceteris paribus problem too - "... the need for ceteris paribus clauses is felt most acutely — and by many felt only at all - when the theory is brought to bear, not on a model, but a real, concrete thing". I think I have remarked often enough on the difference between her interpretation of the role of these clauses (or rather "conditions" in her sense) and a model-theoretic account of their role. She gives as reason for this claim concerning ceteris paribus clauses the fact that the movement from (theory to) model to aspect of reality is in terms of diminishing generality, which strengthens my criticism in this regard. I have pointed out often that the process of concretising has to do with the suspension of these clauses and neither with formulating more and more of them or with "activating" them.

Cartwright illustrates (Cartwright, 1994c: 296 - 297) her account of this aspect of the scientific process as follows:

We begin with a very detailed law; any highly homogeneous fused quartz 
gyroscope placed in space near the earth, electrostatically suspended by a layer of superconducting material, will experience a cerlain amount of precession due to spin-orbit coupling. Presumably some regularity of this kind would obtain if only enough details of the experimental design were filled in; that after all, is what the experimenters are at pains to ensure ... Is [this] highly detailed 'phenomenological' law ... indeed a deductive consequence of the general laws (given the descriptions of the experimental argument), or not? I think not. I find it instructive to break the reasoning process into two chunks:

- (i) the derivation of the behaviour in an abstract diagrammatic model (the simulacra of the simulacrum model of How the laws of physics lie), a process elaborating Schiff's original observation [remark] that a spinning gyroscope should experience a shift in its precession due to the space-time curvature predicted in the vicinity of the earth by the general theory of relativity;

- (ii) the elaborate fitting of this diagrammatic model to a real gyroscope spinning in real space. The full details of this latter will have taken more than twenty years of effor by a large team of experimental physicists and technicians.

At both stages approximation and fudges and totally fictitious 'idealisations' of the kinds described in How the laws of physics lie occur.

The fact however remains that Schiff noticed that it is possible to deduce from Einstein's general relativity theory that a spinning gyroscope should expenience a shift in its precession. The movement here is thus - despite, or perhaps rather as a result of the "totally fictitious idealisations" applied - from Einstein's general relativity theory to Schiff's model, to a real gyroscope.

\subsection{The process of science revisited}

A last few remarks on the confirmation or succession of theories, to illustrate both Cartwright's and my own interpretation of the role of models in the scientific process. Rueger and Sharp (1996: 99) very adequately formulate the two basic choices about the nature of 
our theories with which Cartwright's model of explanation leaves us as follows: our theories are either

- $\quad$ simple and explanatory (in the sense of being classificatory with a wide scope), but not confirmable; or

- complicated and descriptive (phenomenological, with a narrow scope), but confirmable. As I take these not as options for theories, but rather as characteristic of the different stages of the scientific process, 1 shall just point out that maybe some philosophers of science concentrate too exclusively on one aspect of the scientific process. Cartwright feels that philosophy of science is often too close to being exclusively philosophy of scientific theories to be able to offer a balanced view of the process of science (physics); and I agree, although I do not advocate getting rid of the role of theories as part of the referential process of science. Alan Musgrave (1981: 381) claims that we "... do not falsify a theory containing a domain assumption by showing that this assumption is not true of some situations ..., we merely show that that assumption is not applicable to that situation in the first place" in other words, finding a model in which a certain theory is false, does not necessarily imply the disconfirmation of the theory, at first it implies no more than that the theory is simply not applicable in that model.

Blaming the model rather than the theory, might sound indeed like using ceteris paribus clauses to protect theories against refutation. However, there is nothing contrived (or ceteris paribus for that matter) about this at all, it simply is the way science works (from a modeltheoretic point of view, at least). For instance, the fact that it is extremely difficult to use Newton's laws to detemine the route and behaviour of Neurath's bill blown about by the wind in St Stephen's Square (Cartwright, 1994b: 283 - 285) does not really say anything negative about Newton's laws, except that it is too difficult to apply them under certain circumstances. ${ }^{t 68}$ The negative factor is rather that science then seems to have very little to say about wind-blown bills, which also is not really true, it is simply that this is a complex enough situation to warrant a new arrangement of abstractions, and so some kind of new formulation of a combination of certain fundamental laws, which might then lead to the 
construction of new models or amendments of old ones, which, in their tum, might turn out to be empirically adequate of the windswept bill, or whatever situation is being considered.

More often than not when the question of the continuity of science and the nature of the succession of theories in physics is addressed, the example of classical mechanics vs. quantum mechanics and relativistic mechanics is used. Let us briefly take a look, in Cartwright's terms, at the implications this example has for the role of models in the progress of science. Classical mechanics is usually viewed as a limiting case of relativistic and quantum mechanics. "Limiting case" here should be taken as meaning "an approximation to the new theory when its domain is limited to the domain where the older theory was (still is, for that matter) successful". Specifically, the equations of special relativity theory are taken to reduce to those of classical mechanics or non-relativist mechanics in the limit where the speed of light goes to infinity (which implies that there is very little that differs in the equations of these two theories if small velocities are being treated). And also then the equations of quantum mechanics are supposed to take on the form of those of classical mechanics in the limit where the quantum of action becomes negligible, as it is taken to do in the situation of macroscopic objects, Now, although the notion of limiting cases is rather useful in the modeltheoretic approach, even if perhaps mainly in practical terms of saving the time and effort that repeating the experimental activity involved in recreating the "old" theory's evidential base would cost scientists ${ }^{169}$, the notion of approximation is too "naive" in a sense to really fit in easily enough (as mentioned before). It creates the illusion of a prionness, in the sense of a definite controllable march towards final truth, which is just not part of a model-theoretic account of theories. The "hit and miss"-quality of the construction of models is then somehow lost ${ }^{170}$, and that is one of the best sources of scientific creativity, I think.

This kind of approach in terms of approximations is also part of the heritage of the covering law model via the notion of "inference to the best explanation", as Cartwright (1991b: 6) points out in her article Can wholism reconcile the inaccuracy of theory with the accuracy of prodiction? She offers the fact that Newton's laws being able to explain Kepler's laws is taken to argue for the truth of Newton's laws (by inference to the best explanation) as 
illustration of how closely this notion's formulation resembles that of the fallacy of affirming the consequent. That is another illustration of why her arguments against the covering law account are also directed against Adolf Grünbaum's (1954) generic-specific account of laws, because in his terms, Kepler's laws are the specific version of Newton's in the domain of planetary motion - "[s]o to establish Kepler's laws is to establish a case of Newton's laws; and the more encompassing laws of Newton are, conversely, the generalisations of what is (almost) literally true in the orbits of the planets" (Cartwright, 1991a: 6). Again, I think it necessary to point out that this view is, in principle, not against any of the implications of a model-theoretic approach, although care should be taken not to interpret the notion of approximation too naively.

\subsection{The "abstract" and the "concrete"}

The special relationship between theories and models, and models and aspects of reality is illustrated very well by Cartwright's characterisation of models in terms of fables (Fables and models, 1986). She starts the article with the following statement about her aims - the same as in How the laws of physics lie, simply given from a different perspective: "I want to defend the view that the [phenomenological] laws may be true, literally true, yet they need not introduce new properties into nature. The properties they mention are already there; the new concepts just give a more abstract name to them ... we have no need to look for a single concrete way in which all the cases that fall under the same predicate resemble each other. What we need to understand, in order to understand the way scientific laws fit the world, is the relationship of the abstract to the concrete ..." (Cartwright, 1986: 56, 57 ).

The most important point about her discussion of the abstract and the concrete in this article (Fables and mode/s (1986)) is the conclusion (already mentioned above) that laws or theories may somehow be said to be true (although she nowhere spells out that this actually means true-in-a-model), but they cannot be universal. ${ }^{171}$ She (1986: 58 ) says: "We need not assume that they [the theories, or fundamental laws] are at work everywhere, undertying and determining what is going on. If they apply only in very special circumstances, then perhaps 
they are true just where we see them operate successfully - in the artificial environments of our laboratories, our high-tech firms, ...".

Well, I agree that we need not believe that our scientific theories give us laws that are "underlying and determining what is going on". However, our bridges remain untouched by most storms, our aeroplanes do fly around the planet, our astronauts do walk on the moon, and so on and so on. The interpretative development of scientific theories - from the theory to its interpretative models, and from there, possibly, via empirical models to some aspect of reality - as I see it, is a far more contingent issue than some philosophers would maybe care to admit - or be able to handle. I agree with Cartwright about speaking of truth only in terms of models - or the "artificial environments" we create for our theories' application although I cannot see why that should prohibit me from keeping in the discussion something like Van Fraassen's (1980) notion of empirical adequacy to describe the nature of the "last jump" from interpretative model via empirical model to the aspect of reality concerned.

Concentrating on — in my terms - the "jump" or movement from theory to interpretative model, she (in Cartwright, 1986: 58) quotes Gotthold Lessing's characterisation of fables as having the ability or potential to fill in the "graspable, intuitive content for abstract, symbolic statements". This comes close to my view of things, in the sense that my model-theoretic approach may be applied to general statemerits of all kinds, be they symbolic (in both natural and mathematical language), purely mathematical, or simply in natural language (think of morals like "the grass is always greener on the other side"). ${ }^{173}$

Lessing (according to Cartwight, 1986: 59) also claimed that in order to make "a genera! symbolic conclusion" as clear as possible, it has to be reduced to the particular, so that it can be known "intuitively". That is what he meant when he said the general can only become graphic or intuitive (anschauend) in the particular. (All very Aristotelian of course, with which I also have no quarrel.) Note that there is an ontological (the general exists only in the particular; where "exists" means "to be realised, and seen possibly to refer to some real system"), as well as an epistemological (the general becomes graphic, anschauend only in the particular; referning to conditions of truth - or empirical adequacy for that matter) claim 
being made here. Both these claims are addressed in my model-theoretic type of approach to science by the staggering of the various levels of scientific development, as well as by consideration of the nature of the relations and linkages between these levels.

Cartwright (1986: $62-65$ ) discusses Newton's second law of motion: $F=m a$, in these terms. This law is portrayed as an abstract "truth" - maybe theory would be a better choice of words - relative to claims about forces, motions, and masses. As it indeed is, and in the following way:

(1) to be subject to a certain force, say $F$, is an abstract property;

(2) $F=$ ma further claims that whatever object has the property in (1), also has the property of having a mass and acceleration, the product of which gives the value $F$.

Scientists then have to figure out the nature of the typical situation in which real objects have these features. This implies that they have to figure out how to "fill in" the value(s) of each variable (see Cartwright, 1986: $62 \& 63$ for detailed discussion of this example) and then to construct a model in which each of the variables will behave according to their natures - e.g. "... we have the small mass $m$ located at distance $r$ from the larger mass $M$. Now we can look to see if the small mass moves with an acceleration $G M I r^{2}$ (since $G M I r^{2}=F / m$ ). If it does, we have a model for Newton's law" (Cartwright, 1986: 63).

She goes on (ibid.: 64) to set out exactly what I also accept as my view about the nature of theoretical concepts, whether expressed in natural or mathematical language, as being abstract: "On my account, force is to be regarded as an abstract concept. It exists only in the more specific forms to which it is led back via models ... It is not a new, separate property, different from any of the arrangements which exhibit it. In each case, being in this arrangement - e.g. being located a distance $r$ from another massive body - is what it is to be subject to the appropriate force ${ }^{n}$. In other words, the abstract notions we are dealing with in scientific theories are not something over and above their concrete realisations - they do not in any way have "a life of their own" so to speak, floating around somewhere in some special kind of reality, they are merely part of the tools (Cartwright's point again in The toolbox of science (1995)) we use to formulate knowledge (or scientific) claims. The point is 
then simply that viewing the laws of physics as general (formal) claims and their concepts as abstract (and symbolic), comes down to acknowledging that Newton's $F=$ ma can be true of precisely those systems that it treats successfully. It however does not necessarily mean (Cartwright, 1986: 67) that Newton has discovered a fundamental structure goveming all of nature. Again, Cartwright's point about theories being true in limited domains, but not universal is illustrated.

Interestingly enough, this also shows the inaptness of a point Steve Clarke (1998) seems to want to emphasise; namely that there is some kind of tendency among realists (granted, he refers to fundamental realists, but my remark still is worthwhile, given the overall slant of his approach) to believe that one day, somehow, all fundamental laws will have been discovered and then the nagging open-endedness of the tiresome ceteris paribus clauses can forever be closed. Within a model-theoretic approach the notion of such tendencies becomes merely nonsense, since ceteris paribus clauses are not really relevant as such when theories are interpreted. The only kind of "open-endedness" at issue in the process of science, is the open-endedness of abstract linguistic expressions (which may contain ceteris paribus clauses - if any are indeed present) in terms of their potential to be interpreted by various different conceptual models, and, moreover, it is a result of this open-endedness that it is indeed possible within a model-theoretic scheme to link the fundamental laws to reality (or aspects of reality at least).

The open-endedness that is referred to here can thus never be "closed" since that would mean turning to at least a non-statement approach as far as scientific theories are concerned, as well as considerably weakening the realist claims still possible in such a context. Which is, again, why I see the model-theoretic approach as more sensible than Cartwright's account of models. Although she opens the possibility that the models may successfully describe reality, the fundamental laws themselves simply have nothing to say about reality. I find it a bit strange, that both Clarke and Cartwright agree that models "manifest" the fundamental laws, but still seem unable to recognise the simple formal feature of transitivity at play here: if models manifest theories, and models describe aspects of reality, how can theories have 
nothing to say about reality? Moreover, Cartwright herself points out on more than one occasion that "[t]o grant that a law is true - even a law of 'basic' physics ... is far from admitting that it is universal, that it holds everywhere and governs in all domains" (Cartwright, 1994b: 281), which means that she does see some kind of relation between theory, model and reality, but simply not a universal (a prion) one - or not?

In Nature's capacities and their measurement (1989), she works out the relations between abstractions, idealisations, and concretisations in far more detail, because, there, she discusses these notions in terms of causes and their complexities, and in terms of the capacities of nature. More about that in the next section.

\subsection{Nature's capacities causally explained}

Cartwright amends her simulacrum account of explanation in her book entitled Nature's capacities and their measurement (1989) by arguing that causal claims should play a central role in the explanations offered by science. This is an abvious continuation of her attack on the Humean characterisation of science (according to which explaining a phenomenon means showing it to be an instance of a general law or regularity), which she keeps focussed on arguments against the Humean attempt to reduce causal concepts to law-like ones (and finally to reduce these to regulanities, or statements of association). She offers, instead, a metaphysics of enduring causal capacities ${ }^{174}$. She (1995c: 292) claims that "t]laws in the conventional regularity sense ... must be constructed, and the knowledge that aids this construction is not itself again a report of some actual or possible regularities. It is rather knowledge about the capacities of [nature] and what these capacities can do if assembled and regulated in appropriate ways".

Cartwright and John Dupré in their article entitled Probability and causality: Why Hume and indeterminism don't mix (1988: 521) see events (constant conjunctions) in a completely un-Humean way: "... events and things have causal capacities: in virtue of the properties they possess, they have the power to bring about other events or states. ... The Humean tradition downplays capacities, and conceives of them as no more than misleading ways of referring 
to law like regularities. We [Cartwright and Dupré] want to reverse this idea: it is better to think of law-like regulanties as misleading ways of referring to the exercise of capacities". Causality, in the Humean tradition, is depicted in terms of relations between events, which holds, as I have already pointed out, in virtue of the regular association of the empirically distinguishable properties of these events. Dupré and Cartwright (1988: 521), however, point out that, on the other hand, no "right" sort of connections exist between capacities and properties of events. "Capacities are carried by properties. That is, you cannot have the capacity without having one of the right properties. But the same property can carry mixed capacities, and so the true complexity of the situation cannot be revealed by the association of properties". And, moreover, since "... at any stage in [an] inquiry, there are always alternative sets of capacity that could account for the statistical data [under consideration]" (Cartwright \& Dupré, 1988: 522), it is not possible - contrary to Hume - to find statistical data that can "settle" the truth of probable cases of regularity, In line with her empiricist's sympathies, Cartwright wishes to show that capacities can, however indeed, be measured. ${ }^{175}$ She lengthily discusses the use of probabilities in this regard in quite a few of her more recent articles, as well as in Nature's capacities and their measurement (1989). ${ }^{176}$

Cartwright gives (1989: 226 - 227) three main arguments for the real existence of capacities: (In the scope of this thesis I am primarily interested in the second argument, given its relation to realist considerations.)

- She bases the first argument on the nature of the composition of causes and the fact that scientific explanations and predictions involve causes as well as their behaviour.

- The second argument is based on the problem of the exportability of information or knowledge, that is, the fact that information gathered in one situation can be applied in a completely different situation. This is essentially the issue that Poincaré referred to as the problem of "transduction".

- Thirdly, she offers an argument that is a counter-attack on the Humean tendency to "modalise away" capacities, and here she concentrates on the problems of interaction between causes themselves and between causes and capacities, and also on the 
"duality" of capacities, which refers to the problem of controlling multiple capacities associable with one and the same feature of nature.

One of the most important reasons why Cartwright insists on the reality of causes is, as

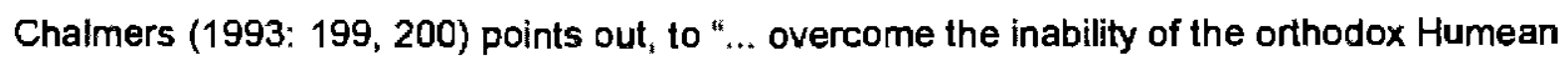
view of laws as constant conjunctions to accommodate the asymmetries that exist between the phenomena constantly conjoined...". "I agree with Hacking", writes Cartwright (1983: 3), "that when we can manipulate our theoretical entities in fine and detailed ways to intervene in other processes, then we have the best evidence possible for our claims about what they can and cannot do". So, she offers the fact that scientists can "successfully intervene" in nature by "manipulating causes" as an argument for the existence of those causes. This point is echoed by Eman McMullin (Cartwright, 1989: 185): "The unordered world of nature is a tangle of causal lines; there is no hope of a 'firm science' unless one can somehow simplify the tangle by eliminating or otherwise neutralising, the causal lines which impede, or complicate, the action of the factors one is trying to sort out ...".

However, as she points out (Cartwright, 1991a: 9), "[t]he punchline of course is that the fundamental laws of physics may not be so fundamental either. ... By choice and arrangement of materials and either by intensive shielding or very heavy over-determination, we create special environments which hold fixed the principal effective parts. We may in this way arrive at very precise and reliable regularities without in any way grasping the true form of what is going on". Cartwright, of course, is here referring to her notion of a "patchwork of laws" - a world of "... tens of thousands of patches, cut up in no particularly logical way, exhibiting tens of thousands of different regulanties of countless different forms ..." (Cartwright, 1994b), which leaves open the possibility of reality being completely unordered. Two brief related remarks on this last statement: I do not see how the acceptance of a notion as metaphysical as the notion of capacities, can soive any of Cartwright's anti-realist worries. On the other hand, the exact nature of the things in reality that scientific theories may be "about" - whether referred to simply as the activities or behaviour of real phenomena, as the mechanisms of reality, the capacities of nature, or whatever else - is irrelevant to the 
successful application of the model-theoretic model of science that I offer.

The primary problem that she wants to solve with her notion of capacities is indeed whether - and to what extent - the laws of physics that are true in certain situations - that is in the "highly contrived environments of a laboratory or inside the housing of a modem technological device" (Cartwright, 1994b: 281, 282) - carry across to "systems, even systems of very much the same kind, in different and less regulated settings" (ibid.). She says "[t]he overall programme I want to urge is a careful and detailed philosophical slory of the evidence about the boundaries of relevance ... for any of our ... fundamental laws. We have to allow for the possibility that they are true but not universal; exact but limited in range" (Cartwright, 1994c: 293).

She sets out a hierarchy of laws in Nature's capacities and their measurement (1989) and also in her Precis of 'Nature's capacities and their measurement' (1995a), in terms of generality - or modality, because the claims at the highest two levels are universal in time and space and they support counterfactuals, license inferences, and so on. Her classification looks roughly as follows:

- At the highest level there are "capacity claims" (Cartwright, 1989: 228), which are to be taken as being general causal claims. These are statements which associate capacities with properties. These claims summanise the range of outcomes that some system can cause. She says about these most general kinds of causal statement that maintain that the most general causal claims - like 'aspinins relieve headaches' or 'electromagnetic forces cause motions perpendicular to the line of action' - are best rendered as ascriptions of capacity" (Cartwright, 1989: 141). So, for instance, aspinins, because of being just that, can cure headaches. The phrase "because of being aspirins" indicates that this claim expresses a fact about a special property, namely that the property of being an aspirin camies the capacity of relieving headaches.

- At the middle level there are "causal claims" that give phenomenological (actual) content to the capacity claims - they are laws about "what singular causings occur in what circumstances what percentage of the time ${ }^{n}$ (Cartwright, 1989: 228). In other words, they 
are more specific than capacity claims and they describe - usually probabilistically what causal claims obtain in some given specific situation. And, thus they can - other than capacity claims - be used in a mechanical way to make predictions. Also, they can be established inductively, as long as the situation they are prescribing for, or predicting of, is the situation they are describing in the first place. "They describe what would happen were the situation like that. But by their very nature they do not describe what would happen were the situation different" - (Cartwright, 1995a: 154) - their weakness lies in their strength.

- At the lowest level of abstraction, at the level of the real, that is, there are singular causal claims. "Nature's capacities argues that it is not possible to characterise correctly the relation between probabilities and causal laws without referring to singular causal facts" (Cartwright, 1995a: 153). That is actually one of the main reasons why this book is still very important from a model-theoretic point of view. Because, if it is true that, as she claims now, fundamental laws are about the capacities of nature, as Chalmers (Chalmers, 1993: 201) points out, then they cannot describe sequences of events as well, and therefore they cannot anymore be taken to lie in the sense of How the laws of physics lie (1983).

The fact that capacities enable us to carry over information gathered in one set of circumstances to another, is the reason why capacity claims are not simply "higher levels of modality, but instead must be taken as ascriptions of something real "(Cartwright, 1989: 158). I will briefly discuss what she has to say on this point, as it points to her realist tendencies with regard to capacities, while she remains anti-realist with regard to fundamental (abstract) laws. The positivist programme for causality is described by Cartwright $(1989: 160)$ as follows:

At the level of modality:

Ascriptions of capacity (meta linguistic summaries of facts about causal laws)

Causal laws (summaries of facts about non-causal laws)

Functional and probabilistic (non-causal) laws 
At a non-modal level:

\section{Occurrent regularities}

Within this system, Cartwright (1989: 160) points out, the claims at a higher level constrain the nature of the set of facts at a lower level and in this way license inferences from one kind of fact at a lower level directly to another without the need for any support from the lower level. She states that this modal view of capacities runs into problems in two areas:

- ceteris paribus conditions involving interactions, and

- multiple capacities associated with the same feature of some (real) phenomenon (and trying to control them somehow).

Looking at the first area, the following. These kinds of assumptions about capacities that is, for them to remain intact from one situation to another - obviously go a long way towards seeing capacities as "things" in reality. But what if the capacity in question is not exhibited in this way? Cartwright claims $(1989: 163)$ that the most common reason for capacities not to exhibit themselves in new situations lies in the influence of causal interaction - the property carrying the capacity interacts with some particular feature of a new situation and so the nature of the capacity changes. She $(1989: 163,164)$ acknowledges that she cannot amend the positivist hierarchy of causal laws without admitting interactions into the descriptive content of reality. The ceteris paribus conditions on inferences licensed by causal laws cannot be specified without already involving notions of causality. Causal claims can function as a "tool to get new causal information out of the old" (Cartwright, 1989: 161), that is, causal laws can function as inference licenses from one probabilistic law to another, but only if, ceteris paribus, the individual singular causes are "right". This ceteris paribus condition involves a notion of causality which is at the same - or a higher - level than the one from which there is being argued. (See Cartwright, 1989: 160, 161 for a formal discussion of these issues.)

Thus, in the context of capacities, this means then (Cartwright, 1989: 164) that "[C]ausal interactions are interactions of causal capacities, and they cannot be picked out unless capacities themselves can be recognised. The attempt to 'modalise away' the capacities 
requires some independent characterisation of interactions; and there is no general noncircular account available to do the job". She claims that rather, independent evidence that some interaction between certain variables and no others - or at a certain level and no other - is occurring, should be found. She illustrates the value of this with the example of chemistry (Cartwright, 1989: 165): "One does not say the acid and the base interact because they behave differently together from the way they behave separately; rather we [should see that we] understand already a good deal about how the separate capacities work and why they should interfere with each other in just the way they do". Thus the ceteris paribus conditions describe the model in which the fundamental ascriptions of capacity will be true. In a model-theoretic account no ceteris paribus clause has the power to describe a model of some theory. Rather models are determined - among other things - by the whole linguistic expression of the theory (including factors such as the nature of the language in which the set of sentences comprising the theory are formulated) as well as by some of the applicationary aims scientists have in mind for the theory. Cartwright's aim is to show that the regularities that are described by the (phenomenological) laws in the model are the consequences and not the sources of these models.

She sees (Cartwright, 1995c: 290) "natures [or capacities] as primary and behaviours, even very regular behaviours, as derivative. Regular behaviour denives from the repeated triggering of determinate systems whose nature stays fixed long enough to manifest itself in the resulting regulanity". Well, as pointed out before, in model-theoretic terms the (relevant) empincal model that "drives" the final references of some theory (via its conceptual model(s)) to some real system is very much dependent both on the nature of the conceptual model of which it is a substructure, and on the nature of the system in reality it shows the theory to be "about".

Looking at the second problem area (the fact that multiple capacities may be associated with the same feature of some phenomenon in reality), the following. The connection between causes and probabilities that causal claims make possible can be described in the following terms: a cause increases the probability of its effect (while obviously a preventative should 
lessen it) if all other causes are held fixed somehow. However, Cartwright argues (1989, throughout chapter 3) that more important than holding the other causes and their forces of possible interference fixed, is that the operation or activity of all other capacities that may be at work, should be held fixed as well, whether they are attached to the cause being scrutinised or to the olher separate causal factors. She claims that only in this way, can facts about capacities be connected to facts about probabilities - "An ascription of a capacity cannot be taken merely as an inference ticket to get from one fact about probabilities to another, that is, as an efficient summary of complicated facts about the pattern of pure probabilities; for the pattern it summarises is not a pattern invoking just the probabilities themselves but a more variegated pattern involving both probabilities and capacities in an essential way" (Cartwright, 1989: 167).

However, since ascriptions of capacities are found in fundamental laws, Cartwright has to say something more about the role of fundamental laws as ascriptions of capacity in the causal account of scientific explanation that she is working out. She (ibid.: 175) writes: "Im]ore important for my thesis, however, is not the fact that laws which are nearly true, albeit for particular situations and finite periods, are not fundamental [which is what Mill also points out $]_{\mathrm{i}}$ but rather that fundamental laws are not true, nor nearly true, nor true for the most part. That is because fundamental laws are laws about distinct 'atomic' causes and their separate effects; but when causes occur in nature they occur, not separately, but in combination". As pointed out before, these "complex combinations" that we find in Nature are of no particular consequence to a model-theoretic realist at all. We have other laws that tell us how to combine effects - e.g. the addition of speeds (which by the way is different for Einstein than for Newton).

Now, Cartwright (1989: 178) has been arguing all the while in Nature's capacities and their measurement (1989) that scientific methodology and its application presupposes that these capacities (or tendencies, as Mill calls them, or propensities as Popper calls them) are real. In other words, the only way in which fundamental laws can be taken to say something about reality, is if they are viewed as ascriptions of capacity. Although this doesn't change 
their nature, they still lie (according to her) however, because they are still Aristotelian abstractions. But, now, at least, they can be interpreted in some kind of realist terms. I fail to see the need for this. Whether the function of the fundamental laws of nature is to assign stable capacities to specific causes (Cartwright, 1989: 179) or not, does not really decide whether these laws may have something to say about reality or not. Rather, it is the ways in which specific models may be shown to be linked to certain aspects of reality that hold that promise. More about this below in Section 4.7.

But, if capacities are real (more than high-level Humean modalities), what becomes of the lower level modal laws, in particular, what becomes of Hume's laws of association? Cartwright (1989: 181) concludes that Nature in no way presents us with them as "given", but rather Nature selects the capacities of different entities and determines their interaction.

It is not the laws that are fundamental, but rather the capacities ... Whatever associations occur in nature arise as a consequence of the actions of these more fundamental capacities ... Nature, as it usually occurs, is a changing mix of different causes, coming and going, a stable pattern of associations can emerge only when the mix is pinned down over some period or in some place. Indeed, where is it that we really do see associations that have the kind of permanence that could entitle them to be called law-like? [Only in ancient astronomy or in science laboratories.] ... laws of association are in fact quite uncommon in nature, and should not be seen as fundamental to how it operates. They are only fundamental to us, for they are one of the principal tools that we can use to learn about nature's capacities.

In these terms then, the basic laws of physics are not laws about sequents of events (à la Hume) but laws about the capacities of nature. I have no quarrel with remarking that "a stable pattem of association can emerge only when the mix is pinned down over some period or in some place", because that fits my description of the functions of models and the ways in which they interact with theories on the one hand, and with aspects of reality on the other hand. Keep in mind here too that the conditions in which capacities "reveal themselves in 
their canonical behaviour are usually in no sense normal at all; [but it] ... requires the highly arlificial and contrived environment of a laboratory to manifest them" (Cartwright, 1989: 200).

Alan Chalmers (1993: 201) agrees with Cartwright that, if laws are supposed to describe capacities, then they cannot be taken to describe Humean sequences of events as well. But, then, fundamental laws do not really lie in the sense of How the laws of physics lie, because only laws taken in the sense of regularities could be said to lie in this sense (Chalmers, 1993: 204). Which is close to what is worrying me, although I do not necessarily see scientific theories in terms of discovenes of regulanties, because it is simply not necessary to think in that way, given the descriptions of the nature of theories and models and their interaction in my model-theoretic system. Moreover, we all know that science has never aimed at describing reality in all its complexity and fullness. The main problem in realist philosophy of science has obviously atways been, in a sense, to link the simplified versions of reality modelled by scientists to fit their theories to the reality of reality as it were. Cartwright (and Roy Bhaskar) offer us ways in which to do this with their various descriptions of the "exportation of information" or bridging the "unsoundness argument". More about this in Chapter 5. Cartwright actually does say something along these lines when she claims (1989: 184) that "... abstractions can be taken as claims about capacities; ... and where abstraction reigns, ... laws - in the conventional empiricist sense - have no fundamental role to play in scientific theory".

\subsection{The "abstract" and the "concrete" revisited}

Fundamental lews or abstractions, considered as capacity claims, have the following distinct features:

- Cartwright calls the abstractions made in science material abstractions (which refers to the fact that there is no a prion recipe for getting from these abstract fundamental laws to the concrete situations (and capacities) they are supposed to be about). This brings in the role of ceteris panibus clauses again, since the suspension of these clauses in my terms "puts back" what abstraction has "left out". 
- In Cartwright's terms the laws that constitute the phenomenological context of capacity claims are causal, and neither laws of association nor equations. In a model-theoretic system the nature of these "laws" may be causal or not. They should merely be specific enough to function within the idealised contextual framework of the conceptual models of the theory in question.

What does it mean to "link" an abstract fundamental law making some kind of claim about the capacities of nature, to reality? How can a general ascription of capacity like that be linked with different situations in reality? Cartwright (1989: 203) discusses these matters in terms of what she calls "idealisation" and "abstraction" on the one hand, and "concretisation" on the other. She formulates the most pressing worries about these processes in terms of the problem of material abstraction.

Concretisation has to do with the route "downwards" (from abstract law to concrete real situation) and involves adding corrections to allow for the effects of interfering or disturbing causes that may be at work in a given real situation, because, as pointed out many times before, causes are rarely single and never act separately in nature. These corrections are a necessity, also in my terms. They are however not ceteris paribus but rather their function is similar to that of boundary and initial conditions. It is a necessary feature of the process of science, model-theoretically interpreted, that these "corrections" are needed, given the abstract and simple nature of fundamental laws (or scientific theories) and the rich changing nature of facts in reality (whether this richness is the result of multiple interacting causes or of something else). So, Cartwright (1989: 184), is not wrong in pointing out that "... the converse processes of abstraction and concretisation have no content unless a rich onlology of competing capacities and disturbances are presupposed", but my point is that that is true, however we fill in the ontology of reality.

The process of idealisation starts in reality, or rather, with some aspect of reality (Cartwright, 1989: 187), and rearranges (conceptually) some of its (inconvenient) irrelevant features. However, note that the model still says something - albeit something more simplified than is the case in reality - about all relevant factors present in the real system 
focussed on, and leaves out only the irrelevant factors. The ideal model thus still has a link with reality, because it sets the aspect being studied in a concrete situation. It is more "real" because all relevant factors are present (even if they are, as pointed out, idealised and simplified versions of the real ones). However, these models also have an "unreal" side to them - they are after all representations, conceptual models about some aspect of reality.

The laws active in models are phenomenological ones (as pointed out in the beginning of this chapter). These kinds of laws are however problematical in Cartwright's terms, in the sense that they are apparently subject to a kind of ceteris paribus condition - they tell us what happens if the relevant factors are arranged in a specific (ideal) way. The (fundamental) laws describing the content of abstractions, however, do not have these ceteris paribus-like conditions. I disagree as I have often pointed out. The fundamental laws are not about everything. Newton's laws of motion and his law of gravitation were not about everything in the universe, but were rather concemed with everything to do with gravitation. For this reason it is (some) fundamental laws that need ceteris paribus clauses and not the phenomenological ones. The ideal character of the models is ideal because the theory has to be true in it, and not because of special ceteris paribus conditions. The only sense in which "all other things are equal" in a model is in the sense of satisfying the truth relations between the model and its theory. This means that only entities and relations to which the (language of the) theory refers (under the relevant interpretation) occur in the model, and there they behave as the theory stipulates.

Cartwright (1989: 190, 191) claims her notion of abstraction to be more Aristotelian than Duhemian. For Duhem an abstraction offers scientists images of concrete instruments manipulated in reality and images of schematic models of those instruments, constituted with the help of theoretical instruments. Scientists then reason about these images and apply the laws of science to them. In model-theoretic terms a cycle of the scientific process starts off in reality and although the mediation of models - Duhem's images perhaps - are necessary to trace science back to reality, it still is claimed that science is about real systems and not oniy about these images. Duhem ${ }^{177}$ - rightly this time - claims that if there is 
correspondence between an abstract symbol and a concrete fact, it can never be in terms of "complete parity". And, moreover, a single theoretical symbol may correspond to a variety of concrete facts such that these facts underdetermine the possible interpretations of the theoretical symbol in question.

For Aristotle, on the other hand, abstraction means subtraction as it does for both Cartwright and me. Aristotle (quoted in Cartwright, 1989: 197) sees the scientific process starting off with a concrete "particular" with all its properties. Then "... we strip away — in our imagination - all that is irrelevant to the concerns of the moment and focus on some single property or set of properties 'as if they were separate"' (ibid.). In other words, other than Duhem, Aristotle believes - like I do, and Cartwright sometimes seems not to - that the objects we study are really there to begin with. Abstractions thus do not imply that the real aspect of reality science focuses on is changed such that the departure from truth is "imperceptibly small", but rather, as Cartwright (1989: 186) puts it, abstraction implies subtraction in the sense of disregarding certain factors that are of less importance for realising the aims of the envisaged theory than others.

Now, for Cartwright it is phenomenological laws that offer the descriptions of real objects. Cartwright (1989: 225) writes: "... if we could write [the phenomenological laws] down, [they] would literally describe the features of this concrete phenomenon and the nomological links within it. [Such a law] would be a highly complex law, and would include a specific description of the physical structure and surroundings of the concrete device. Possibly it could not be written down; perhaps the features that are relevant to its operation make an open-ended list that cannot be completed. But because we are talking about a concrete object, it is at least conceivable that some law is true of its operation". Actually, there exist no such "open-ended lists" of features relevant to the operation of "highly complex" laws. Such lists are only necessary if one's aim is to describe a system in all its complexity, and although - as pointed out before — that seems to be Cartwright's aim, it is neither my own nor the aim of science in general. Science never tries to describe the features of any concrete phenomenon in all its complexity. No empirical model of a specific real system is ever 
complete - or need ever be complete.

Cartwright (1989: 224) describes a schematic diagram (diagram 5.1 there) of a heliumneon laser and points out that it is taken that the concrete object modelled or pictured in that diagram operates under the same law as the one in the diagram. Of course the diagram offers only a representation of the real system, and is, actually, incomplete in the sense that it is not literally true, but that does not make it as abstract as its higher-level theories, because it does still offers a description of the real system's essential nature. She then (in diagram 5.2) gives a block diagram of a laser (Cartwright, 1989: 225). This diagram is a material abstraction of the real laser, because it does not at all attempt to describe the actual behaviour of any laser, but rather it "tells what a laser does by virtue of its function and arrangement of its parts" (Cartwright, 1989: 226). This diagram is an abstraction from the concrete details of a real laser and focuses on its "underlying principles".

The problem of material abstraction is however - as pointed out before - that there is no universal a prion rule that sets out the movement from the abstract law to the phenomenological content, because the additions and corrections necessanily made by the phenomenological laws depend in each instance on how the abstraction is realised in that particular case.

Cartwright wants to know why or how scientific realists may believe that what happens in an idea/ case is commensurable with real cases? Her (Cartwright, 1989: 190, 191) answer is as follows: "... the logic that uses what happens in ideal circumstances to explain what happens in real ones is the logic of tendencies or capacities. What is an ideal situation for studying a particular factor? It is a situation in which all other 'disturbing" factors are missing (or controlled at least). And what is special about that? When all other disturbances are absent, the factor manifests its power explicitly in its behaviour. ... This tells you something about what will happen in different, mixed circumstances - but only if you assume that the factor has a fixed capacity that it carries from situation to situation".

The naive realist description of the scientific process in terms of approximations, however, conflates two separate actions, namely abstraction and idealisation, which both 
Cartwright and I (although maybe for different reasons) want to keep apart in our interpretations of this process. I assume, on the other hand, that scientific descriptions and explanations given by theories and models can be "carried over" to real situations. This is, rather than being the result of the reality of capacities, because of the way the modeltheoretic system interprets the links between aspects of reality, models and scientific theories, and also, because of the fact that we see these theories being applied - and working - all around us every day.

In Cartwright's case, the focus is simply on kinds of abstraction aiming at formulating laws of capacities, where it is causal factors that are isolated and their capacities that are studied. But what exactly is it that ascriptions of capacities (or fundamental laws or abstract theories) say about real things? In other words, what facts in reality make abstract capacity claims true? Given the hierarchy of laws that Cartwright works with, the answer to this question lies in the nature of the bearing of abstract theories on the more concrete and descriptive laws, that is, the phenomenological laws, that fall under them. It is, however, very difficult to answer these questions satisfactorily, because of the problem of material abstraction, already briefly referred to in the above. At the centre of the process of material abstraction lie activities of correction and addition, which are not ruled (governed, or determined) by any set of universal (a priori) rules. (Which is my point exactly.) These activities are, also, not of the ad hoc nature they are sometimes thought to be by naive scientific realists. Rather, according to Cartwright (1989: 202) they are sufficiently motivated in the sense that they genuinely describe other causes, interferences, and so on, that are active in the particular concrete situation being considered. These correcting activities are necessary, because of the subtractions abstract laws make (when addressing reality for the first time in my terms).

However, as Cartwright points out (1989: 106, 107), to get back to the actual concrete laws that constitute the phenomenological content of these abstractions, the initially omitted factors must be added in again. But, where do these factors come from? Cartwright (1989: 204 - 206) explains that the theory itself provides them, and gives an example (ibid.) to 
illustrate her point, although she also stresses that they are never entirely given by the theory since there will never really be an end to further factors relevant to a particular case. She says (1989: 207): "I have put 'real' in quotes to signal my worry. For I think that, no matter how open-ended the list is, this kind of process will never result in an even approximately correct description of any concrete thing. For the end-point of theory-licensed concretisation is always a law true just in the model".

It seems that Cartwright somehow links the so-called "open-endedness" of the corrective ceteris paribus - in her terms - clauses to the fact that theories can only be true in their models. Again, that is not the model-theoretic conclusion, because of the semantic links in terms of satisfaction functions between linguistic systems and their mathematical structures (conceptual models) and substructures (empirical models). Alan Chalmers (1993: 196) offers the following example in support of Cartwright's views: "The explanation and prediction of the retum of Halley's comet was certainly a triumph for Newtonian theory. Nevertheless, the first 'sighting' of the comet on its most recent return enabled the predicted orbit and time of return to be corrected and subsequent attempts to track it were able to benefit from that correction". The empirical truth of this illustration is of course not disputed. However, I do not see why it necessarily has to be interpreted as implying that theories can only ever explain what happens in models. These corrections were made as a result of what "really" happened real observations - and consequently led to more sophisticated models of Newton's theory, which at the same time are empirically more adequate than previous models.

There is a very fine difference in emphasis between Cartwright's account (of the process of science) and mine, but it is a very important one, because it relates to our attitudes towards the realism of our models. I would prefer to say, rather than simply claiming that theories cannot say anything about the aspect of reality that their models may be linked with, that theories can only explain in that little piece of reality that each of their models "refers" to (or rather might refer to). In other words, taking a previous example: the solar system model of Newtonian mechanics consisting of only seven planets, (used before Neptune was discovered) did indeed refer to the real situation. Although, in reality there were nine planets 
all along, the fact remains that it is quite possible to concentrate only on some of them and not on all at once. Whether and to what degree Newton's laws were empinically adequate when using this "restricted" model might seem to be a more difficult issue to deal with. But it is not really, since I claim - in agreement with Cartwright - that empincal adequacy (or truth) is a notion that can only be used meaningfully if linked with the model offening the relevant interpretation of the theory being considered at the time, and the relevant empinical model available at the time.

\subsection{Conclusion}

Cartwright is very ambiguous in her statements about the links between theory and reality, though I find nothing wrong with the following remark (Cartwright, 1989: 211): "I think we cannot understand what theory says about the world nor how it bears on it until we have come to understand the various kinds of abstraction that occur in science, as well as the converse processes of concretisation that tie these to real material objects". I agree that the context-dependence (or ideal character) of the (abstracted) factors "added back" (after the suspension of ceteris paribus clauses) when the model is constructed should be taken into consideration, but, in my account, this is done by linking the empirical adequacy of the theory with the particular conceptual and empinical model, and not by denying the possibility of any relation between theory and reality.

Cartwright's notions concerning ceteris paribus clauses seem to be at the basis of a lot of the aspects of her work that are unacceptable to me. These notions are exemplified by her treatment of Newton's gravitational law: she enfeebles the absolutely general law by rendering it as if it were saying that (as far as we know) the gravitational force only kicks in when no other forces are around; and she ignores the fact that we know how to add forces and, more generally, in many cases how to merge what different theories are saying about different aspects of the same type of system. Apart from this misguided rendering of fundamental laws, she also misinterprets the reason for the idealised nature of conceptual models as the result of ceteris paribus clauses' stabilising influence. As I have pointed out 
before, the idealised nature of these models is far rather the consequence of the formal aspects of the truth (satisfaction) relations between theories and their models. The focused nature of science has nothing to do with some vicious influence that these clauses have on the enterprises of science, but is simply part of the definition of scientific knowledge.

Another of the main shortcomings of her work lies in the fact that she rarely seems to take the stages of theory formulation into account, as model-theoretic realism (see chapters 2 and 5) does. If the structure of scientific theories is analysed both as far as theoretical formulation and application are concerned, the model-theoretic nature of the act of securing some referential link between some theory's terms and the entities and objects of some system in reality becomes clear and obvious. Moreover science does not aim - as Cartwright seems to think - at offering complete descriptions of particular concrete real systems in all their detail. Even if some system could be isolated - which it cannot be - no scientist is ever interested in $a / /$ its aspects!

A last untenable aspect of Cartwright's views lies in her strong metaphysical leanings as far as proving the referential nature of fundamental laws is concerned. According to her, only if it is taken that fundamental laws refer to capacities of Nature, l.e. that these laws are capacity claims, can they be about something in reality. Model-theoretically speaking, the specific ontological nature of that about which fundamental laws are, is not only not necessarily causal or couched in terms of capacities, but basically irrelevant. In the next chapter the main points of a model-theoretic realism will be discussed and illustrated. 


\section{CHAPTER FIVE: A MODEL-THEORETIC REALSM ${ }^{178}$}

\subsection{Introduction}

The realism issue threads through all of the above. No examination of the nature of scientific theories can be complete without addressing the relations between these theories and reality. In a model-theoretic model of science such as mine the basic ontological assumption made is that science is about something that exists independently of it. This ontological assumption has however as little metaphysical content as is possible. Claiming that reality exists "outside" of human practice neither means that reality is unknowable nor, at the other extreme of the scale, that science simply mirrors it.

To determine whether science is about an independent reality or not, I believe one has to examine both the actions necessary for producing scientific knowledge and those aiming to apply such knowledge. From the previous chapters it should be obvious that I argue that at least some of the terms of a given scientific theory possibly refer to entities and relations of some system in reality if reference has been assigned to these terms by some empinical substructure of some conceptual model of the theory. In other words, a scientific theory can be said to be "about" the potential of entities in some system of reality to give reference to some terms or objects in this way. This potential however, is independent of the existence and knowledge of humans. It is the actualisation or realisation of this potential that requires human action. (See also Chapter 2.)

As has been mentioned before, any successful model of a theory is guided by contingent conditions which are context-specifically constructed. The range or content of these models thus cannot be established a prion, but has to be investigated a posteriori by means of histoncal studies of the development of science. We can however at least say (a prior) that formally, the abstract linguistic character of scientific theories allows the possible existence of (many different) models constructed to interpret them; and that these models (with the help of their empinical substructures) have the ability to realise the potential systems in reality have for possibly giving reference to terms in scientific theories. The human-independent potential of reality and its contingent context-specific realisations are thus conceptually speaking 
fundamentally different, even if they are epistemologically (and methodologically) related. "Reality" and "knowledge of reality" - i.e. ontology and epistemology in their general senses - should not be confused. Thus the historical reality of theories and models is dependent on the actions of humans, while the reality of the ongoing potential of systems in reality to give reference to the terms in these theories and models is not. The need for acknowledging the conceptual stratification of the conceptual development of science should now be even more obvious, since at the level of the theory alone, all that is apparent is the multiinterpretability of the theory and thus the possibility of it finally referring to a variety of systems in reality.

In The Scientific Image (1980), Bas Van Fraassen remarks that realists believe scientific theories offer "a faithful replica, in all cetail, of our world". I am arguing (in contrast to Van Fraassen's straw man-realist) for a view of realism that is more sophisticated in the sense that the intricacies of the various semantic relations between reality - in terms of real systems - and scientific theories are taken into account and it is acknowledged that absolute universal statements about these relations fit as uncomfortably within a scientific realist framework as in any other framework. ${ }^{179}$ The identification (discovery) of particular instances of the general terms of theories becomes possible only through interpretations of these general terms, such as mass, momentum, and so on, in context-particular models of the theories.

As acknowledged above, the possibility of discrepancies between theories, models and actual systems in reality has to be acknowledged and as Nancy Cartwright, Ronald Giere and their allies nightly advocate, the need for particular conditions to make "real" sense of general theories has to be accepted too. But this neither leads necessanily, for instance, to Van Fraassen's notion of empirical adequacy concerning only the phenomena, nor to typical scientific realist claims that real systems are approximately captured by models of theories.

I simply claim that there may possibly be a set of models of some theory which fits some systems of reality and add that it is via historical studies concentrating on the context-specific models already constructed to interpret the relevant theories - and perhaps by constructing 
some new ones - that this can be determined. It is this inherent feature of science - that is the multi-interpretability of its theories - which should be emphasised if a sophisticated realist interpretation of the conceptual development of science is offered. And the multiinterpretability of scientific theories is the counterpart of the abstract nature of science. In this sense, it seems natural that it is so difficult to describe the relations between science and reality in simple terms and impossible to give one foolproof set of rules or recipes for theories to refer to reality.

Somehow Ronald Giere's (in Churchland \& Hooker, 1985: 82) remark that man is not the measure of all things but rather the measurer, seems very fitting in this sense. The structure of science's development hangs necessanily together with its theoretical nature. The verification procedures for the content of science however are never given beforehand by fixed rules, but are a result of the context-specific actions and constructions of human scientists. Notions like "constructed reality" and "real science" should therefore not be viewed as representing opposing interpretations of science, but should rather be accepted as the two poles of one very complex time-bound relationship,

\subsection{Reality and science}

Model-theoretically speaking science studies systems in reality. This refers to the abstracting simplifying nature of science. As already often pointed out in the above, no-one, not even scientists, can study reality in all its fullness at once. Not only do scientists focus on some particular system of phenomena in reality, but also they aim to "adjust" that system in such a way that they can focus only on certain of its features. The kind of abstraction that Cartwright talks about and that I have discussed in a model-theoretic context in Chapter 2 is a necessary and sufficient condition for scientific knowledge. If certain abstractions are made from the richness of expenences that reality has to offer, scientific knowledge of the real system in question becomes possible. And, vice versa, if some knowledge claim is offered as part of science, the nature of that claim will (relative to the complexity of the universe) be simple and it will be about a sufficiently abstract version of some real system (even if that 
system is the cosmos!).

Reality is not unknowable, but rather only knowable in a certain way. I am aware that this sounds particularly Kantian, but that is entirely my purpose. The basis of a modeltheoretic methodology is Kantian in the sense that it implies that we can only know reality through science, but that scientific knowledge can only be achieved through certain abstractive actions. However, the Kantian Ding-an-sich is the reality we study via science. There is no other "underlying mechanism" or anything else that is somehow so fundamental to the ontology of reality that we cannot know it. Knowing through abstraction is knowing. There is no other kind of knowing, scientifically speaking. And, moreover, this kind of knowing is adequate in the sense that it does indeed allow us to study, discover, and utilise knowledge about reality.

Any kind of realism that decides how reality has to be in order for science to be possible - such as that of Bhaskar (1978) - is too metaphysical for my taste. I have been arguing all along - and pointed out many times, see Section 2.1 for instance - that a modeltheoretic realism is one that focuses more on science than it does on reality. Connecting an ontology of science to an ontology of reality however often seems to be strangely lurking behind many philosophic accounts of science. It seems as if Cartwright (1989), for one, has been seduced. Her hierarchy of "capacities" somehow seems to be offered as a kind of mirror image of her hierarchy of scientific laws - fundamental laws are capacity claims, phenomenological laws depict causal relations in reality, and the experimental stage of science focuses on "singular causings". Roy Bhaskar $(1979,1981)$ also seems to have been caught, albeit perhaps in a different, but not necessanily lesser, way. In Bhaskar's terms the mechanisms of reality form part of the lowest - ontologically deepest - level of reality and their scientific counterparts are causal laws. The actual events produced by these mecharisms still form part of the "intransitive" (real) dimension of science but are conducive to the identification of constant conjunctions or patterns of events at the "transitive" dimension of science.

From the above - as has already been implied in the introduction to Chapter 2 - it is 
obvious that I plead for a distinction between ontology and epistemology with regard to science. I am aware that philosophers such as Joseph Margolis (1995) deny that this is possible. The reasoning behind disclaiming that such a distinction is possible seems to me as mainly based on the notion of science as a social construction. This is another of many contemporary echo's of Bachelard's (1934) point that it is difficult to see how science can be said to be about a human-independent reality if our notion of reality seems to be so very dependent on human action. One of the problems here, I think, is a certain vagueness of terminology.

In trying to clarify this confusion, let us first make it clear that in model-theoretic terms science is indeed also an individual and social construction. Science is "transitive" in Bhaskar's sense as against the "intransitivity" of reality. Scientific knowledge can change and is contingent on the actions of scientists formulating it. However the notion of "reality" is sometimes used as a group noun to refer to the immediate "stuff" or results of scientific knowledge. That is, the idealised pictures of the "external" world that science offers us are somehow seen as constructing a reality about which science is. Since these "images" are also still changeable because they are part of science, it is then perhaps concluded that reality too is changeable and therefore that a scientific epistemology is necessarily linked to an ontology of this (scientific) reality. ${ }^{1 B D}$ The "immediate" pictures that models of scientific theories offer of some system in reality are however just that - i.e. representations of reality. The reality that is independent of science, in the sense that it exists regardless of whether its processes have already been "discovered", "explained", or "described" by science, is perhaps better denoted by the - somewhat outdated - term "Nature". Reality in this sense is not dependent on human actions at all (except in so far as it encompasses humans and their actions or technology based on science). It is a complex system of "mechanisms" the processes of which continue now as they have done through the ages. And, it is the ontology of reality in this sense that is separate from scientific epistemological factors. The "reality" of science is an idealised version of this "Nature" and consists of already established theories and the various actions of scientists as well as the results of these actions. This "reality" is 
a social construction and this is the reality the more moderate constructivists claim to be constructing, in the sense that science cannot be studied without taking these matters - i.e. the human activities and their context-dependent mativation driving the scientific process into account.

The ontology of this "scientific reality" and the epistemology of its generator - l.e. science - can indeed not be separated. In a model-theoretic account of science this becomes even more clear. The models that offer us these pictures are an integral part of the process of science. The set of conceptual models referred to as the "intended" models of scientific theories in the above, are shaped by the already established pictures of this kind. And, moreover, at the interpretative stage of science the justification for, and evaluation of, scientific theories are offered and carried out within the context of this "scientific reality". The point is however, that in the final instance, although science is "social" and "constructivist" it is not a reflexive enterpise in the usual social constructivist meaning of the word. Science is not about something that it has constructed itself and that is inherently of the same nature as science itself. Science is about "Nature" and about discovering the intricacies of the mechanisms according to which "Nature" operates. In this sense, as pointed out above, ontology - in the sense of the ontology of that about which science is - and epistemology have to be separated.

The "game" of post-Kuhnian philosophy of science has one trick that has to be leamt if the realist quest is to be salvaged. This is the trick of keeping constant one of the changing factors at issue (i.e. "Nature" in this case) while acknowledging the variability of all the (transitive) others involved. Bhaskar (1978) claims that the relationship between science and reality seems problematic only if one either accepts the social character of science, but denies that its object of study is independent of all social activity (the so-called "epistemic fallacy'), or if one accepts the independence of reality, but denies the social nature of science (the so-called "ontic fallacy"). ${ }^{\text {ind }}$ A model-theoretic realism is not guilty of either of these fallacies. He (Bhaskar, 1989) depicts the basis of a valid realist philosophy of science in terms of a choice between either an epistemological or an ontological relativism. He chooses 
an epistemological relativism and refers (Bhaskar, 1989: 57 ) to it as the "... correct thesis of epistemic relativity, which asserts that all beliefs are socially produced, so that all knowledge is transient, and neither truth values nor criteria for rationality exist outside historical time ...". To ontological relativism he (ibid.) refers as the "... incorrect thesis of judgemental relativism, which asserts that all beliefs are equally valid, in the sense that there can be no (rational) grounds for preferring one to the other".

Making the choice - as a model-theorist would also do - for Bhaskar's epistemological relativism, and thus acknowledging that truth criteria as well as criteria for (scientific) rationalism are part of the philosophy of science, does not mean that any criterium goes, but rather the opposite. The construction of these kinds of scientific criteria is, model-theoretically speaking, undeniably a function of the conceptual and empirical models of scientific theories. Validating them is the function of the various semantic relations that exist between reality as "Nature" and these models.

Before we tum to a closer examination of these relations, a last few remarks on science as it is depicted in model-theoretic terms. Around 1960 philosophers of science found themselves in somewhat of a crisis. The logical empinicist inspired way of thinking about scientific knowledge as being the "crowning achievement" of human reason suddenly seemed horribly empty. It began to seem less than clear what exactly it is that determines the line of research science should pursue at a given time, and, consequently, to determine which scientific theories are finally getting at "the truth". Philosophers of science became obsessed with questions like "What do we really know?", "What should we believe?", "What is evidence?", "What are good reasons?", "Is science as rational as people used to think?". These questions lead to serious uncertainty about the nature of reality: "What is the world?", "What kinds of things are in it?", "What is 'true' of them?", "What is 'truth' in this sense?", "Are the entities postulated by theoretical physics real, or only constructs of scientists' minds for organising expeniments, or worse, simply figments of their fertile imaginations that come in handy when they start formulating theories?".

Philosophers of science have been divided over the answers to these questions for 
centuries. During the sixties - and ever since - the debate between realists and anti-realists has become far more intense though because of the strong historical and social flavour of the specific crisis they found themselves in. The opening phrases of Kuhn's The structure of scientific revolutions (1970) decided the context of this debate: "History, if viewed as a repository for more than anecdote or chronology, could produce a decisive transformation in the image of science by which we are now possessed" (Kuhn, 1970: 1). Part of the background of this debate ${ }^{182}$ is the old division between confirmation or verification and falsification. Carnap, for instance, tried to explicate verification in terms of a theory of confirmation because he believed that knowledge has rational foundations, while Popper held that rationality consists in (critical) method, and that our (scientific) knowledge is fallible.

As Hacking (1983: 5) however points out in his book Representing and intervening (1983), these philosophers also share something, that is, they share the image of science that Kuhn's work rejected. They both thought (Hacking, 1983: 5,6) - among other things that

- there is a clear division between observation and theory;

- the growth of scientific knowledge is cumulative;

- $\quad$ science has a pretty strict deductive structure;

- $\quad$ scientific terminology ought to be as precise as possible;

- there is a distinct and fundamental difference between the context of justification and the context of discovery.

Kuhn disagreed with all of the above and stressed that above all science is essentially historical.

My arguments in this section thus far imply that scientific actions - and their authority - are not about Nature in the traditional confirmational sense of satisfying some set of atemporal methodological rules and offering a body of neutral pure objective data about reality. But, neither do the entire scientific enterprise and its products offer simply sets of (false) context-specific data. Rather, science is about "Nature" in the sense that it is a system of knowledge claims that operates according to a set of contingent rules and offers a body 
of equally contingent ${ }^{183}$ data about systems in reality that offers us "snapshots" of "Nature".

\subsection{A modified image of science}

Let us look again at the features of the image of science that Popper and Carnap share, but which are unacceptable to Kuhn, as they are accommodated by a realist model-theoretic account of science.

\subsubsection{The distinction between observation and theory}

I think it is obvious that a model-theoretic realism implies that observational and experimental actions and actions of formulating and applying models and theories take place at different stages or levels of the scientific process, although they constantly complement each other, Abstractions based on established and new observations play a major role at the beginning of the process when the intended model of some future theory is being constructed. Observational results also direct - together with the thematic preferences and other specific factors guiding the interpretations of scientists at this stage - the transition from the intended model to the theory in the sense of the interactive conceptual movement between this model and the selected aspect of reality. In formulating the theory - and thus fixing its class of possible models - the intended model, but also the variety of features in reality that are to be dealt with by the theory and its models, must be taken into account. During the interpretative stage of science observation again plays a definite role in the form of experimental data embodied in empirical substructures of conceptual models of the theory in question. Throughout all of these stages it has to be noted though that established theoretical frameworks - especially those concerning the interpretation of experiments and the use of instruments - are never separate from observational activities.

Observation thus permeates the whole process of science, and moreover, the old fixed distinction between "observational" and "theoretical" terms dissolves. ${ }^{184}$ Theoretical terms such as mass, electron, force - are neither empty metaphysical results of the linguistic formulation of scientific theories, nor can their reference be "fixed" once and for all by the so- 
called bridge relations that are supposed to give them "observational" content. Rather, the meaning and reference of these terms slowly emerge duning the vanious interpretative stages of science via the conceptual and empirical models of the theories containing them.

Dilworth expresses related ideas. He (Dilworth, 1994c: 155) writes:

In seeking to understand why the laws of science take the form that they do, the scientist attempts to conceive what the reality underlying them must be like. He thus constructs an idealised model, which has as its source that which he feels he does understand ... The model should be constructed so as to depict a physically possible, albeit idealised, reality, whose existence would naturally manifest itself in the laws requiring explanation. ... a scientific model represents an ontology the nature of which may be taken as being responsible for the epistemology we associate with scientific laws. And ... the very fact that an explanation of such laws should be tentative necessarily suggests that that aspect of reality which is responsible for them is not open to direct inspection. Thus, if we consider models which depict these (perhaps temporarily) hidden aspects of reality as constructing the essence of scientific theories, we can charactense theoretical terms as terms used in referring to those entities in the real world (should they exist) as they are depicted in such models.

\subsubsection{Science is cumulative}

I have already discussed this issue in Chapters 3 and 5 . Briefly, the following. The metaphor of a clock ${ }^{195}$ nicely describes my view of the way in which science can indeed be said to be cumulative. Take the hour-hand to represent scientific theories, the minute-hand to represent the various context-specific models, and the second-hand to represent empirical data at the level of reality. I claim that the process of science is similar to the speed of the hands of the clock: theories change very slowly, models more quickly, and empirical data (observations etc.) the quickest, while definitive relations hold between these three aspects of science. (Maybe shifting to a new disciplinary matrix is analogous to buying a new clock.) 
Each model of a given scientific theory is subjected to extremely strict testing against reality - by whatever form of testing is applicable to the model in question - and it is seldom that a model withstands these tests without at least being modified in some way, i.e. without the intention shifting to some other model(s) (of the same theory) with better empirical fit. Theory changes usually occur only when the possibility of changing and modifying the relevant models of the theory concerned has been exhausted. But even if a specific model of a theory is "discarded" the possibility of transforming that model and using it for other purposes in another interpretation of the same (or another) theory always exists. (Think of epicycles - the precursor of Fourier analysis - and the ether — the precursor of fields in space-time.) Thus the different levels of the scientific process are inherently related and it is this fact that guarantees the continuity of scientific knowledge. ${ }^{186}$

\subsubsection{The deductive structure of science}

Well, this is a difficult one. In my terms, a theory consists of a deductively closed set of sentences (in some appropriate language) ${ }^{187}$, i.e. the theory contains all the sentences deducible from the axioms of the theory. In model-theoretic terms, the generalising activities of abstraction that take place at the beginning stages of the formulation process of a scientific theory are in direct contrast to the particularising activities of interpretation duning the applicatory stages of science.

\subsubsection{Scientific terminology}

Scientific terminology tries to be as precise as possible, simply in the sense that it would seem a bit funny to be using vague ambiguous terms to formulate so many hours of detailed exacting research. But, if by "precise" is meant something like "having only one obvious absolute meaning or interpretation", I cannot agree, because such an approach totally ignores the model-theoretic implications of the scientific theory as a generalising statement. I am referring here to the various interpretations possible of one scientific theory by virtue of its abstract nature. The meaning and use of scientific terminology are also linked to specific 
models and are thus perhaps more contingent than some philosophers would like, since theoretical terminology has model-specific meaning and reference.

\subsubsection{The contexts of justification and discovery}

I agree with Kuhn that these contexts cannot really be separated. Even if they are taken in the traditional sense of the context of justification being about and using so-called "internal" features of theories, and the context of discovery being about and using factors "external" to scientific theories, the constant interaction and overlap between these contexts during the process of science become obvious in model-theoretic terms. It is possible to see that justification will perhaps play a larger role in the movement from the theory via its various models to systems in reality, than in the initial development from an aspect of reality to the theory via the intended model. And, surely one could say that discovery will be very important in the initial stages of theory formulation, but so will it be in the final examining of systems of reality giving reference to the terms in the model(s) of some theory. Again, it is the interconnectedness of these stages or levels that makes it very difficult to separate these contexts.

It is however undeniable that both have important roles to fulfil in the process of science as a whole, as is specifically illustrated when the question of the truth of a theory in some conceptual model is examined. Scientists work within the context of the logic of justification when they construct the conditions and structure of the interpretation they work from in such a way that the theory (being the deductively justified consequences of axioms) has the best possible chance of being true under that interpretation. However, they work in the context of the logic of discovery when they determine the "truth" or "empirical adequacy" of the interpretation itself — that is the "fit" of the interpretation to some real system - because here they have to do with a multitude of different (often entertwined) tests, varying from the empirical to the mathematical according to the specific interpretation they work from. More on the model-theoretic notion of empirical adequacy in Section 5.4. 


\subsection{The empirical interpretations of scientific theories}

\section{4 .1 Introduction}

The different accounts of science offered by the various non-statement approaches to the status of scientific theories all offer - among other things - variations on the theme of scientific realism. The model-theoretic tools these views are equipped with seem to offer a very good chance of, on the one hand showing that there are, indeed, certain empirical relations between the terms of theories and the entities in real systems, and an the other hand, defining the nature of these relations more and more precisely. I shall first discuss these issues in sections 5.4.2 and 5.4.3.

Finally I shall, against the background of these discussions, analyse some "traditional realist problems". Typically the main "problematic" areas of a realist approach to science are the following. The problem of explaining the success of science, given the realist notion of approximate truth, lies in what Worrall (1994: xviii) refers to as "the argument from radical changes". This comes down to the problem of explaining — or justifying - the "approximate truth" of theoretical concepts that (supposedly) have been "discarded" in the progression of science - for instance "ether". A second related problem is to be found in the issue of underdetermination of theories by empirical data. How can the same theory be applicable to — or explain even - more than one phenomenon (or group of phenomena) if theories are "approximately true"? Finally, there is a problem, typical of many varieties of scientific realism, concerning the "additional" metaphysical content that theories that "really refer" seemingly need - given that it is often implied that mere empirical results, i.e. theories having the "right empirical consequences", are not "enough" to justify these forms of realism's existential claims regarding the phenomena their theories are "about" ${ }^{\mathrm{t}}{ }^{\mathrm{g}}$

\subsubsection{Empirical adequacy}

The main question that one tends to want to answer in a realist context is, of course, how exactly scientific theories "get to" reality. The problem is that there is no simple answer to that question. The conclusion drawn from claims like these - e.g. "there is no final 
description of the links between scientific theories and reality such that theories invariably refer to "something" in reality" - should however not be that therefore realism is untenable. A model-theoretic approach to realism shows us exactly that, and more importantly, such a realism offers us the tools to examine - and make sense of - the various and complex empincal links between scientific theories and real systems. It may seem that - perhaps as a result of the many-to-many relations between theories and their conceptual and empinical models, as well as between these models and systems in reality - model-theoretically one merely ends up in the empinical substructures of some conceptual model of a given theory, rather than "in reality". Well, what does it mean to be "in touch" with reality? I cannot see it meaning anylhing more than being in touch with the empirical practices of science. And, that is precisely what the empirical models embedded into a given theory's conceptual model(s) allow us to do. Of course, they allow this in a conceptual way, but then, the content of science - i.e. the set of its knowledge claims - is conceptual too is it not? A model-theoretic realism is thus a realism about objects in reality and the relations between them, although conceptual models (and the empinical models isomorphically embedded into them) are used to describe real systems by describing the systems' objects and the relations between these objects.

In structuralist terms, if the data concerning the real system in question can be conceptualised in terms of a potential model of the theory in question, that is, if the data satisfy the framework conditions of the theory, then some empirical claim in terms of the theory can be made concerning the specific "range of phenomena" in question. Such an assertion will be empincally testable and so it may be shown that the real system in question also satisfies the empirical laws of the theory. This then will imply that the potential model of the theory is indeed an actual model of the said theory, and thus that this model is an element of the theory core. And then it may be concluded that the real system in question offers (at least) one application of the theory in question.

In my terms, the structuralist notion of a "potential model" might be viewed as one of the conceptual models of a certain theory. The notion of an "actual model" may be very close to my notion of an "empirical model" since roughly it seems that they do the same work. In 
model-theoretic terms, if it can be shown that a conceptual model of a given theory has an empirical substructure in which the experimental and observational dala conceming a certain real system are conceplualised (or represented), the theory may be said to refer to the real system in question. The "empirical claim" that in structuralist terms proclaims a certain potential model of a theory to be an actual model of the theory is, in my terms, represented by the various model-theoretic relations between the theory, its conceptual model, its empinical models, and some real system. This notion of showing a potential model to be an actual model of a theory also reminds of Suppe's definition of empirical truth in terms of which the class of causally possible systems is claimed to be the theory-induced class of systems. (See Chapter 3.)

Van Fraassen considers a theory to be empincally adequate if some model of the theory is such that (real) structures describable in experimental and measurement reports are isomorphic to an empirical substructure of the relevant model of the theary.

In terms of Beth's state-space approach the link to reality is given via some satisfaction function between some (mathematical) state-space describing some physical system, and a set of elementary statements concemed with physical measurements. This means that the actual state of a physical system at a certain time may be given by defining some state-space representing the possible states of that system and some satisfaction function, which holds if the actual state of the system (described by some elementary statement) is an element in the domain of the relevant state-space.

In Suppe's terms a theory is empirically true if the semantic relations holding between the propositions in the theory-formulation ${ }^{489}$ language and the class of causally possible physical systems are those that hold in the case of theory-induced physical systems as well. If a physical system is in the class of theory-induced systems, the domain of the physical system will be a subset of the domain of the theory, and the sequence of stales of that physical system will be determined by the theory's laws. This implies that propositions in the theory-formulation language may refer to and describe some physical system because a physical system in the theory-induced class of systems restricts the theory to a single 
sequence of states.

Van Fraassen's constructive empiricism (Van Fraassen, 1980) denies the need for dwelling on questions concerning reference between theoretical entities and aspects of reality. According to this view, relations of empirical adequacy between phenomena and "empirical substructures" of models of theories that refer to "observational" terms of theories, are sufficient for the needs of philosophy of science.

Let us briefly examine the distinction that Newton made between "apparent motion" and "absolute or real or true motion" as Van Fraassen (1976: 624ff., 1980:44ff.) sets it out. In Ptolemy's terms the earth is stationary. In Copernicus's terms the sun is stationary. In Newton's terms neither the sun nor the earth is stationary. Planetary motion in Newtonian celestial mechanics is observed relative to the earth's motion. The notion of apparent motion is introduced such that the apparent motion of particular bodies accounts for the differences of their "true" motions (Van Fraassen, 1976: 624). Ptolemy need not have made the distinction between true and apparent motion, since to him "true" motion was exactly what was observed. In Copemican terms we can only observe the planets' motion relative to the earth, which is not stationary and thus the apparent motion of the planets are the difference between the earth's true motion and the true motion of the planets. Newton generalised apparent motion - i.e. motion relative to the earth - to the motion of one body relative to another. Any observed motion thus became a relative motion and an apparent motion is motion relative to an observer (Van Fraassen, 1980: 45).

Newton separated the reality he "postulated" from the phenomena he "saved" (Van Fraassen, 1980: 44) by referring to the "absolute magnitudes" of his axioms and to their experimental determination as "sensible measures" (ibid.). Apparent motions "form" (ibid.) relational structures defined by measuning relative distances, time intervals, and angles of separation. These relational structures Van Fraassen (ibid.) calls "appearances". In a mathematical model of Newton's theory bodies are located in Absolute Space in which they have "true" motion (ibid.). Van Fraassen (1976: 624) writes: "[b]ut within these models we can define structures that are meant to be exact reflections of ... appearances and are, as 
Newton says, identifiable as differences between true motions. These structures, defined in terms of the relative relations between absolute locations and absolute times ... I shall call motions ...". The notion of "appearance $5^{\mathrm{N}}$ thus refers to the actual observed motions - the "phenomena" — while the notion of "motions" refers to the terms of some mathematical model of Newtonian celestial mechanics.

Van Fraassen (1980: 45) continues "[w]hen Newton claims empirical adequacy for his theory, he is claiming that his theory has some model such that all actual appearances are identifiable with (isomorphic to) motions in the model". Thus, in Van Fraassen's and my terms $s_{1}$ empinical adequacy - in Newton's terms - would mean that all empinical models (substructures) of Newton's theory will be isomorphically embedded in the particular (conceptual) model defined above. However, as both Van Fraassen and I stress, all that is really necessary or possible for determining the empincal adequacy of a given theory, is that it has at least one (conceptual) model with an embedded empirical model (adequately empirically related to some real system).

In model-theoretic terms the models of the theory should thus indeed be adequate to the phenomena, but if the fact that the theory may be "adequate" to (true in) its (conceptual) models is taken into account as well, we have a model-theoretic realism that addresses the possible meaning and reference of "theoretical entities" without relapsing into the metaphysics typical of the usual scientific realist approaches. Remember that the deductive structure of theories is mirrored semantically in the (conceptual) models of the theory and thus cannot be represented fully by looking only at empincal substructures of these models. ${ }^{180}$ Surely in terms of the above, it is the appearances that allow us to make sense of the motions (via the relevant models) which in their turn allow us to apply Newton's theory in a certain way?

A clear manifestation of the empirical link between a model and some system in reality is given by the dimensional analysis in terms of the basic units in a derived physical quantity. According to the Oxford concise science dictionary (1996), a unit is the "specified measure of a physical quantity such as length [e.g. centimetre], mass [e.g. gram], time 
[e.g. seconds], etc., specified multiples of which are used to express magnitudes of that physical quantity" (ibid.: 751). The basic units of physical quantities are multiplied and divided to get derived units with dimensions, e.g. a unit of the form $L^{p} M^{q} T^{r}$, where $L, M$, and $T$ indicate length, mass, and time, respectively, and $p, q$ and $r$ are (usually) integers. Examples: length (distance): $L=L^{1} M^{0} T^{0} ;$ mass: $M=L^{0} M^{1} T^{0}$; time: $T=L^{0} M^{0} T^{1} ;$ frequency, that is "per time": $1 \pi=L^{0} M^{0} T^{-1}$; speed: $L T=L^{1} M^{0} T^{-1}$; acceleration: $(U T) / T=L^{1} M^{0} T^{-2}$; momentum, that is mass $\times$ speed: $M(U T)=L^{1} M^{1} T^{-1}$; force, that is mass $\times$ acceleration: $M\left(U T^{2}\right)=L^{1} M^{1} T^{-2}$; energy, that is, work, that is momentum $\times$ speed $=$ force $\times$ distance: $L^{2} M^{i} T^{-2}$; action, that is momentum $*$ distance $=$ energy $\times$ time: $L^{2} M^{1} T^{-1}$; power, that is force $x$ dislance $\div$ time $=$ work per time: $L^{2} M^{1} T^{3}$. The definitions (given in conceptual models of the measurement theory in question) of the basic units (and hence of the derived units) link these units empincally (calculations given by some empinical substructure of the conceptual model in question) to certain very definite aspects of reality. A second is the duration of 9192631770 periods of a certain specific radiation emitted by a caesium-133 atom (that is the radialion corresponding to the transition between hyperfine levels of the ground state of this atom). A centimetre is the length of the path travelled by light in a vacuum during a time interval of $1 /\left(2.99792458 \times 10^{10}\right)$ second. A gram is one-thousandth of the mass of a certain platinum-iridium object kept by the International Bureau of Weights and Measures at Sèvres, near Paris in France.

As shown above, the science of measurement (metrology) offers some of the clearest examples of the empinical relations between models and aspects of reality. In physics too we have extraordinarily accurate theories. Penrose (1997: 51) writes: "In quantum field theory, which is the combination of quantum mechanics with Maxwell's electrodynamics and Einstein's Special Theory of relativity, there are effects which can be computed to be accurate to about one part in $10^{11}$. Specifically, in a set of units known as 'Dirac units', the magnetic moment of the electron is predicted to be $1.001159652(46)$, compared with the experimentally determined value of $1.0011596521(93)^{\circ}$. This last instance also shows that the highly regulated results of experimental situations may indeed be "carried over" to the "complexities" 
of reality, quite successfully and without too much ado. This is the subject of the next section.

\subsubsection{Transduction}

The problem of transduction refers to the problem of "carrying over" the results of some idealised (experimental in this case) context to the complexities of real systems. Bhaskar stresses the fact that scientists produce the empirical grounds for the laws of nature in their laboratories, but not the laws themselves. (In my terms this refers to the distinction between science, the "reality" of science, and "Nature" that I discussed in the first sections of this chapter.) He (Bhaskar, 1986: 30) claims that distinguishing between "real and universal ... but non-empirical laws and their real and empirical but contextually localised grounds" dissolves the problem of transduction. The justification for each individual law can thus be found in the Bhaskarian stratification of nature, and not by tying laws to closed systems and ceteris paribus conditions (as "classical" empincists seem to do). ${ }^{191}$ Cartwright (1995a: 155) echoes this when she claims that scientists should "... figure out how to combine laws together and how to cash out ceteris paribus conditions ...". This has to be done, given her problem of material abstraction, against the material conditions of the situation in question, and the only way in which this is possible, she claims, is to assume the existence of capacities. A modeltheoretic realism needs neither a Bhaskarian analysis of reality nor Cartwright's notion of capacities to make sense of the experimental situation and its links to real systems.

Cartwright (1989: 181) concludes that Nature in no way presents us with its laws as "given", but rather Nature selects the capacities of different entities and determines their interaction. "It is not the laws that are fundamental, but rather the capacities ... laws of association are in fact quite uncommon in nature, and should not be seen as fundamental to how it operates. They are only fundamental to us, for they are one of the principal tools that we can use to learn about nature's capacities ${ }^{n}$ (Cartwright, 1989: 181, 182). The fact remains though that Cartwright's aversion to fundamental laws - and her consequent metaphysics of reality in terms of capacities - show that she does not understand the process of science in model-theoretic terms at all. Fundamental laws can only be said to "lie ${ }^{\mu}$ about real 
situations if science is taken as striving towards some final model via which Nature can be explained and described in all its complexity. If it is however - model-theoretically understood that the reality of science is a constructed one, and moreover, that this fact is the flipside of the abstract nature of science, fundamental laws become an integral part of the process of science and the need for devising some total ontology of reality - as "Nature" disappears. Never can all the ceteris paribus clauses of a given scientific theory be "cashed out" at once, rather the model-theoretic process of science is such that different sets of these clauses are suspended through different models of the same theory, relating the theory to possibly different aspects of reality.

Bhaskar also claims that causal laws are "ontologically uncoupled" (Bhaskar 1986:44) from pattems or sequents of events. Empirical regularities only occur as a result of active interference in nature: therefore this ontological distinction - between the empirical regulanity that scientists produce (in the transitive dimension) and the causal law (in the intransitive dimension) that it enables us to identify - has to be presupposed and acknowledged if experimental (and thus scientific) activities are to be comprehensible.

If something like Bhaskar's transcendental realist assumptions about the relations between reality and science are not made, any scientist could generate any pattern of events at will, rendening all scientific activity totally uninteresting. In other words, a realist philosophy of science should indeed be able to sustain an epistemological relativity, but fight against surrendering to ontological relativism, as discussed above. As pointed out before, a modeltheoretic realism can - as it should be able to do - accommodate such a Bhaskarian epistemological relativism. The varied nature of the conceptual models of a scientific theory represents precisely such a relativism. A model-theoretic framework however offers besides the fact that, as in the transcendental realist case, no ontological relativism need follow - two additional implications of a realist nature:

- retaining the notion of a scientific theory (as the only means by which all the possibilities, offered by the variety of models, can be encompassed) protects the epistemological choices at the conceptual (model) level from dissolving into a meaningless multiplicity, 
and

- the intemal structure of the relations between the conceptual models of a specific theory and systems in reality prohibits the stark anti-realist implications usually associated with the problem of the underdetermination of theories by data (and models in this framework).

We as philosophers cannot tell which model (of a certain theory) provides the most adequate description of reality, because the ontology which a realist philosophy of science can offer is limited to descriptive claims about the structure of reality, while the epistemology it offers centres around the conceptual structure and development of scientific knowledge. Thus, although something akin to Bhaskar's distinction in terms of transitivity is also assumed in a model-theoretic realism, such a realism need, I stress again, not dwell on the kind of metaphysical musings about the actual structure of reality that both Bhaskar's notion of lawlike mechanisms, and Cartwright's capacities leading to some patchwork of laws, seem to imply. Only science itself can offer us an ontology which can specify the contents of the structures reality contains and the particular ways in which it behaves.

Scientific method should thus ideally provide a model-dependent theory-dependent strategy, because such a strategy offers within a realist context the possibility of modifying or amending our existing models and theories in the light of further research. The methodological principles of a strategy like this will themselves depend on the theoretical picture provided by currently accepted theories. Both our new theories and the methodology by which we develop and apply them depend upon previously acquired theoretical knowledge. And this fact about the continuous nature of science, as well as science's various relations to reality can best be supported and explained by a model-theoretic realist conception of scientific knowledge.

Thus in explaining phenomena a model-theoretic realism tries to show how these phenomena may be embedded - via some empirical model — within a conceptual model of some theory so that any real system exhibiting the phenomena in question may be within the reference of the theory's terms. Patrick Suppes's approach ${ }^{192}$ seems to me to hold the 
most promise as far as solving problems concerned with possible relations between theoretical entities, empirical data, and phenomena go. In my terms, proving the existence of relations of isomorphic embedding between empinical models and conceptual models (which incorporate Suppes's hierarchy of models between, at the highest level, theories of experiments, and the notion of experimental design, closest to reality) offers a way in which to refer to the contingent and complex relations between real systerns and mathematical models of theories in a precise way. These relations can otherwise only be examined and analysed a posteriori as part of historical studies of science.

From this viewpoint Cartwright's recent attacks (1989, 1994a, 1994b, 1995a) on "fundamentalism" seem rather pointless. Very few philosophers of science still try to "close" the open-endedness ${ }^{193}$ characteristic of models (and accompanying ceteris paribus clauses in Cartwright's terms). Cartwright however does seem to have come to other insights lately, in so far as she (Cartwright, 1997) now stresses that pluralism - the view of the sciences that she $(1989,1994 a, 1994 b, 1995 a, 1995 b, 1995 c)$ has been advocating for the past few years - does not necessarily imply anti-realism. She (Cartwright, 1997: 167) writes: "To say the laws of physics are true ceteris paribus is not to deny that they are true. They are just not entirely sovereign". In an approach such as I am offering the abstract nature of theories, the "stabilising" and controlling nature of ceteris paribus clauses, the idealised nature of models, and the model-specific suspension of the said ceteris paribus clauses all fit into and can be accommodated in a logical reconstruction of the scientific process. Such a reconstruction can meaningfully address questions concerning the "truth" of theories, as well as their possible reference to reality.

5.5 The succession of theories, verisimilitude, underdetermination, and other intertheoretic issues

In what follows I shall discuss some of the problems - briefly mentioned in the introduction to this chapter — that are typical of "traditional" realist accounts.

The main realist problem concerning the succession of theories lies in explaining the 
different meanings or interpretations (or references) certain theoretical notions have been given through the history of science. Larry Laudan (in Cushing, Delaney and Gutting, 1984: 90) remarks that realists typically claim that scientific theories are "approximately true" such that their "central" - empirical - terms "genuinely refer" (ibid.) to real (physical) phenomena. This property of approximate truth is then offered as justification or explanation of the success of science. Laudan (ibid.) writes: "Our theories are successful, the realist maintains, precisely because they come close to representing things as they really are". Putnam (1975; 73) refers to this approach to the success of science as the "no miracle" approach. ${ }^{194}$

Laudan (ibid.) puts his finger on the problem when he comments (ibid.) that we - as realists - know very well that our theories are not "true simpliciter", but at the same time the "truthlikeness" of our theories cannot be left uncommented on. The fundamental flaw underlying the notion of approximate truth $^{\text {t95 }}$ lies in its very definition. There simply is no reason to believe that approximately true scientific theories necessarily will be successful scientific theories, or vice versa. ${ }^{150}$ (The study of the verisimilitude of theories is a highly technical area. More on this a little later on in this section.)

Now Laudan (in Cushing , Delaney, and Gutting, 1984: 92) points out two altemative ways in which to examine the success of science. The first is an examination of the specific semantic features of scientific theories that allow these theories to be so successful (ibid.). The second alternative lies in "epistemic and methodological questions" conceming the "selection procedures which scientists use for picking out theories with impressive credentials":197 (ibid.). It seems, however, as if Laudan (ibid.) feels that realism has very little to say on these issues. A model-theoretic realism however can - and obviously does address both these issues, albeit perhaps with not exactly the kind of slant that Laudan wishes for.

As far as the semantic features of successful theories are concerned, a model-theoretic realism cannot offer a prescriptive discussion of these features. Rather, it explains how the semantic relations of theories in terms of their conceptual models may be used to "track" the "real" referents of these theories. Such a realism is thus descriptive in the sense of claiming 
that a successful scientific theory will have such-and-such semantic features, rather than being prescriptive in the sense of claiming that a theory with such-and-such semantic features will definitely be successful. The reason for this again lies in the fact that this model of realism is a context- or model- specific realism in the sense that no absolute discussion of the empirical links between conceptual models of theories and the systems in reality is offered, and moreover, as Van Fraassen (1980) too points out, all the data concerning all phenomena will never be "in" at a given time. With regard to the second alternative Laudan mentions, briefly the following. A model-theoretic realism is perhaps the only kind of realism that can indeed say anything about these "selection procedures". While Laudan (1981a, 1981b) sees the answer in the so-called "problem-solving" ability of the theory, model-theoretic realists find the answer in terms of models of the theory and its various links to real systems, as well as to other models of the theory in question.

Jarrett Leplin (1997) offers another - rather familiar — approach to explaining the success of science in realist terms. He offers this approach in terms of a definition of a socalled "novel" prediction with the understanding that a theory that is capable of making such predictions would be a better choice than one who does not have this ability. Briefly, his (ibid.) definition seems to come down to the claim that a prediction is novel for a theory if no information about the predicted phenomenon is necessary for the prediction of that phenomenon by the theory in question, and if there is no other theory that can explain the prediction of this phenomenon.

The idea of "novel" predictions to account for the succession - and the success - of theories has been with us most prominently since Lakatos. ${ }^{98}$ Model-theoretically speaking, his (Lakatos, 1987) distinction between progressive and degenerative research programmes - as well as Leplin's version of this distinction - essentially come down to nothing more than the kinds of changes taking place at the conceptual level of science in terms of the construction of models of theories. A new model of the same theory will be able to make predictions conceming the system in reality the theory is focussed on, that older models (of the same or older theories) could perhaps not make. And whenever it becomes impossible 
to construct a new model in these terms, it is time to make a theory change rather than a model change. Such a "new" theory will then presumably have some models that can make "novel" predictions in the usual sense of these predictions not being contained in the previous theory's models. The validity of science in terms of its conceptual (model) level however tums the notion of "novel" facts into a far less rigid feature of "new" theories than is usually implied.

A model-theoretic realist account of science offers all the tools necessary for the technical study of the "truthlikeness" or "closeness to the truth" of scientific theories. The study of verisimilitude had its beginning with Karl Popper's Conjectures and refutations (1963). This book contained a proposal for a definition of "theory $y$ is closer to the truth (has larger verisimilitude) than theory $x^{\prime \prime}$. It is interesting to note that Popper's approach to theories was firmly in terms of the statement approach - i.e. the view of theories in terms of deductively closed sets of sentences, although the study of the verisimilitude of theories may better be done in a model-theoretic context that has decidedly non-statement characteristics too. In 1974 David Miller and Pavel Tichý showed (independently of each other) that Popper's definition of verisimilitude is not usable, since according to it no two theories containing false sentences are comparable. Development after that has mainly been in the "non-statement" approach - or rather in the spirit of my model-theoretic approach in which both models and theories play their equally essential roles. This work is exemplified by a large number of publications, of which the following is a selection of the more important and representative recent papers: Brink, C. \& J. Heidema (1987), Burger, I.C. \& J. Heidema (1994), Kieseppä, I. (1996), Kuipers, T.A.F. (1987), (1992), and (1997), Niiniluto, I. (1987), and (1998), Oddie, G. (1986), Ryan, M. \& P.Y. Schobbens, (1995), Zwart, S.D. (1998) and Zamora Bonilla, J.P. (1996).

Another interesting problem presents itself in these terms. This is the problem of underdetermination. A model-theoretic interpretation of the occurrence of underdetermination of theories by data (and by models!) offers at least new insights to notions such as scientific progress by accumulation, scientific truth, the problem of reference, and the nature of the relationship between language and reality. (See Chapter 6 for final conclusions in model- 
theoretic terms concerning these notions.)

Traditionally (that is, in terms of the received or statement view of science) the nature of underdetermination has been understood in terms of possible relations of a many-to-one nature existing between phenomena or systems in reality and the observation terms of the theory in question, as well as one-to-many relations between sets of protocol sentences (formed from the observation terms and expressing data) and possible theories incorporating or explaining such a set of protocol sentences - that is, the existence of incompatible but empinically equivalent theories. So empirical data are too incomplete to determine uniquely either a real system, or a model, or a theory. If - as has been explained in more detail in Chapters 2 - the interpretative chain between theories and systems in reality is stretched such that conceplual models are interposed between theories and real systems, the character of the underdetermination "problem" changes positively.

Within a model-theoretic account of science the above mentioned problematic relations are analysed in terms of two sets of relations. The first set is a set of one-to-many relations, and the second is a set of relations that may be many-to-many, many-to-one, or one-to-many relations. The one-to-many relations in the first set are interpretative relations between the terms in some theory and the terms in its various models. These relations assign meaning (and potential reference) to the theoretical terms. The second set of relations in different types of combination exist between the terms of models (or of only one model) and the objects and entities of some real system (or systems).

The realist advantage of a model-theoretic account of science is that it illustrates that the complexity of this last set of relations need neither be circumvented somehow, nor be regarded as sufficient to shoot down any realist aspirations. Retaining the notion of scientific theories as linguistic expressions at the "top" level of science solves the problematic part of the first set of one-to-many relations by the simple fact of the incompleteness ("openendedness") of interpretations of formal languages. The possibility of a given scientific theory being interpreted in more than one mathematical model (structure) is natural in a very basic sense in model-theoretic terms. Postulating the various relations between terms in models 
and aspects of reality also in these terms is perhaps not as basic, but seems to be the obvious and best explanation for the successes of science and consequently the best affirmation of scientific realism. Rather than try to wriggle out of the whole referential mess, advocates of a model-theoretic account of science accept that the relations between the terms of conceptual models and real phenomena cannot be described or defined (or even explained) by some rigid (barren) set of absolute correspondence (bridge) principles.

Model-theoretically speaking theories are not simply underdetermined by data in the familiar Quine-Duhem way, but theories are also underdetermined by their individual models. (The theory is the set of sentences true in all its models. Many different theories are also true in any particular model.) The underdetermination of theories by data is the original problem of explaining the existence of empirically equivalent, yet incompatible, scientific theories. In the history of (the philosophy of) science instances of such theories are quite common think of the various ways in which an electromagnetic field has been described from Faraday through Einstein to Feynman. In general model-theoretic terms the underdetermination of theory by data is not really a problem. Underdetermination becomes a problem only if one believes in some "absolute" semantic content of a theory, which that theory, as syntactic (linguistic) entity, is not able to capture uniquely. Otherwise, if the various thematical factors, such as the traditions within which scientists work, are all taken into account, together with the abstractive nature of science and the interpretative nature of its application, underdetermination of theory by data becomes a necessary feature of science rather than an insurmountable problem. The variety of links between the conceptual models of scientific theories and systems in reality in terms of the empirical models of the theory in question dissolves the usual antirealist implications of the notion of underdetermination. These links can, in terms of a model-theoretic realism, be shown to be valid and, moreover, such a realism shows exactly how scientific theories can still be about real systems even if the objects and entities of these systems are somehow described in different conceptual terms.

As far as the underdetermination of theories by their conceptual models is concerned, briefly the following. The models of a theory say "more" than the theory in the following formal 
sense. The set of sentences true in some model $M$ of a theory $T$ in a first-order language $L$ is larger than T. A theory T is usually incomplete in the sense that there is a sentence $\alpha$ such that neither $\alpha$ nor $\neg \alpha$ is in $T$. A particular model $M$ of theory $T$ is however "complete" in the sense that a model "divides" the language $L$ into true (including $T$ ) and false sentences. The choice of a model for interpreting a particular theory is underdetermined in the sense that such a choice is limited by the empirical model in which certain "observation" sentences are true, but this empirical model is just a (small) substructure of the to be chosen model. This is not an arbitrary choice though, precisely because the model has to "make" the theory's sentences true while keeping true the sentences in $L$ that have been shown to be empirically adequate. Again, this takes out a lot of the strength behind the usual antirealist implications of underdetermination, given the nature of the empinical models of a theory as set out above.

Related to the problem of underdetermination is the question of the various relations that may exist between theories and their models. Is it possible to have one structure that makes (under two interpretations) the sentences of two theories (even in different languages) true? Yes (although maybe "in the limit"); one of the models of Einstein's general theory of relativity in its low velocity low field limit is given by Newtonian mechanics. In general though it should not merely be said that all models of preceding theories are models of limiting cases of succeeding theories. Such an approach trivialises the idea of "limiting cases" to an absurd extent. A model-theoretic realism is not merely about the same things being called by different names. Rather it is about the entire conceptual organisation of objects and relations on them, i.e. of structures, in such a way as to give the best possible explanation for real systems at a given time.

Can the same theory have two models that are both valid? Yes, well that depends on the strength of one's realism I suppose. The model of Newton's theory representing our solar system that consisted of only seven planets before the discovery of Neptune, the one containing eight planets before the discovery of Pluto, and one containing all presently known nine planets in our solar system, may all be constructed as models of Newtonian celestial mechanics. And, moreover, they all refer to certain systems in reality as well. Whether they 
each are as "successful" as the others is a different question, the answer to which will have to be found in terms of the research aims of the specific application. In this context, note that then obviously, the same empirical model may be embedded into different conceptual models of the same theory. For instance, think of the various models of Einstein's equations - they have to be empirically equivalent - which in my sense means that they must have the same empirical model - since they are all about the same aspect of reality, although this shared empinical model is in each interpretation of the Einsteinian equations isomorphically embedded into completely different conceptual models of the general theory of relativity.

Let us very briefly look at the structuralist approach to these problems. They approach the question of underdetermination mostly via what Moulines refers to as "intertheoretical links" ${ }^{\text {199 }}$. Roughly, (Sneed, 1984: 367) "an intertheoretic relation is a one-to-one correspondence $\sigma$ between subsets of the elements of models of $T_{1}$ and $T_{2}$ together with a general set-theoretic relation $\lambda$ and a relation $\rho$ between the potential models $\ldots$ of $T_{1}$ and $T_{2}$ so that in $\rho$-related models ... $\sigma$-corresponding elements are $\lambda$-related. ... thus we may say that elements $e_{1}$ and $e_{2}$ in the models for $T_{1}$ and $T_{2}$ are 'the same' if there is an intertheoretical relation $\langle\sigma, \lambda, \rho\rangle$ between $T_{1}$ and $T_{2}$ so that $\lambda$ is the identity relation on sets and $\sigma\left(e_{1}, e_{2}\right)$ ".

Sneed (1984) remarks that in a case where the purely formal properties of all the theoretical elements of theories are exactly the same - i.e. $M_{P}(T)=M_{P}\left(T^{\prime}\right)-$ but the basic laws of the two theories differ, the meanings of the theoretical elements are different in the different theories. In the non-statement sense in which "giving" the axioms of a theory means "giving" the set of models of the theory in question - i.e. all structures that satisfy these axioms - it is the case that the "meaning" - I prefer "reference" - of theoretical terms will be "different" if the "basic laws" - i.e. axioms — of two theories differ. However since these theories' framework conditions may still be the same — in Sneed's sense of their potential models being identical - I should think that it would still be possible to see continuity between the theories. Be that as it may, a difference in empirical laws resulting in different reference for theoretical terms is surely to be expected? I can only think that the usual underdetermination insecurities are lurking somewhere in Sneed's mind in the sense of 
different sets of empirical laws with identical potential models turning out to have the same partial potential models - i.e. intended applications. Underdetermination's sting however may be much reduced in model-theoretic terms as shall be discussed below.

As example of a case of meaning change for terms referring to theoretical individuals, he (Sneed, 1984: 365 - 366) gives the following analysis of terms referring to electrons in classical electrodynamics and terms refering to electrons in relativistic electrodynamics. According to Sneed (ibid.) the two theories have the same partial potential models but not the same potential models. Well, this agrees with the description of sets of parlial potential models as theory-independent subsets of the set of potential models of the theory. The empirical claim concerning electrons in each case will show each partial potential model as being in the specific theory's core and so "tum" the relevant potential model in question "into" an actual model of the relevant theory. Sneed (1984:366) writes "[a]ssuming the pretheoretical description $I$ of the intended applications and the reduction relation $\rho$ are the same, then the relevant [theoretical individual kind terms] ${ }^{200} \ldots$ can have the same denotation only if the denotation of both lie wholly within the intersection of the contents of $T_{1}$ and $T_{2}$ ".

For instance, Sneed (1984: 361) claims that classical rigid body mechanics ( $T^{\prime}$ ) reduces to Newtonian parlicle mechanics (T) in the following terms (ibid.): T'reduces to $T$ via a reduction relation $p$ in which the individuals of $T^{\prime}-i$. e. ngid bodies - correspond to sets of individuals of $T$ - i.e. particles. By the reduction relation $\rho$, every intended application of $T$ ' (i.e. a member of 1 ) may be conceived as a set of Newtonian parlicles in several ways and are intended applications of T that are not "identified" with nigid bodies. Thus Sneed (1984: 360) writes "[r]oughly, rigid bodies are 'conceptualised' as certain kinds of Newtonian particle systems. Those Newionian particle systems of this kind which, in addition, satisfy the laws of Newtonian particle mechanics 'make up' rigid bodies that satisfy the laws of classical rigid body mechanics".

Two terms can however not be theoretical in both theories (Sneed, 1984: 367). They are either non-theoretic in $T_{1}$ and theoretic in $T_{2}$, or non-theoretic in both. This is kind of obvious, though, as Sneed (1984: 368) explains: "For the kind of intertheoretic relation required for an 
element to be regarded as 'the same' in two theories is just the kind that is a necessary condition for a theory-independent determination of the element." The only way in which the same element may appear theoretical in two different theories is that there are no methods of determining (ibid.) it in either theory. This is entirely possible, although, as Sneed (ibid.) points out no real life examples come to mind, probably, he (ibid.) claims, because the lack of such determination possibilities would make us view such theoretical elements as without any "empirical content" (ibid.). These terms can however be theoretical in both theories in model-theoretic terms. Moreover this can indeed be determined - independent (or not) of each other - in terms of the conceptual interpretations of these terms offered by the various models of the two theories concemed.

This point becomes more obvious in Van Fraassen's terms. Recall that according to him (Van Fraassen, 1976: 631) a theory is empirically adequate if "all appearances are isomorphic to empirical substructures in at least one of its models". Van Fraassen's answer to the implications of the notion of underdetermination is given in terms of the empirical strength of a theory. He (Van Fraassen, 1980: 67) writes: "If for every model M of [theory] T there is a model $M^{\prime}$ of $T^{\prime}$ such that all empirical substructures of $M$ are isomorphic to empirical substructures of $\mathrm{M}^{\prime}$, then $\mathrm{T}$ is empirically at least as strong as $\mathrm{T}^{\prime}[\operatorname{sic}]^{\prime \prime}$ - put in this way it seems rather as if it is $T^{\prime}$ that is empincally at least as strong as $T$. Earlier Van Fraassen (1976: 631) wrote that 'Theories $T$ and $T$ ' [each being as least as strong as the other in the above sense] are empinically equivalent exactly if neither is empirically stronger than the other. In that case ... each is empirically adequate if and only if the other is ${ }^{* 201}$

In these terms he (Van Fraassen, 1980: 59) discusses the "classic" example of underdetermination related to the fact that in classical mechanics all measurements may be reduced to measurements of time and position, and (ibid.: 60) writes: "... if two bodies have different masses and if they were brought near a third body ... they would exhibil different acceleration. But ... there are models of mechanics ... in which a complete specification of the basic observable quantities does not suffice to determine the values of all the other quantities. Thus the same observable phenomena equally fit more than one distinct model 
of the theory". ${ }^{202}$ Van Fraassen $(1980: 60,61)$ explains that the various different treatments of "mass" offered by the axiomatic theories of mechanics developed in this century "are demonstrably empirically equivalent [in his terms of his definition of the term] ... Therefore from the point of view of empirical adequacy, they are indeed equal",

A last remark on underdetermination in model-theoretic terms. Van Fraassen (1980: 63, 64) claims that realists "... wish to play down ... underdetermination, arguing that any precise definition of empirical adequacy and empirical equivalence will lead to the conclusion that a physical theory is completely adequate only if it is true". A model-theoretic realist rather claims that if theories are empirically adequate, it means they are true in certain models the empirical substructures of which conceptualise the empirical data of the relevant real system.

Van Fraassen (ibid.) agrees that physical theories describe "much more than what is observable" (ibid.). However it is still the empirical adequacy of the theory in question that really matters. He (Van Fraassen, 1980: 64) claims that the notion of empinical adequacy does not "collapse" into a mere notion of (metaphysical) truth, since "it relates the theory to the actual phenomena (and not to anything which would happen if the world were different, assertions ... which ... have, to my [Van Fraassen's] mind, no basis in fact but reflect only the background theories with which they operate)" (ibid.). The point that Van Fraassen wants to make here is, I think, that empirical adequacy offers us a way in which we can delimit all the talk about the various possible models of some theory so that we can show one of these models to be actually about some real system. Van Fraassen of course would only agree that it can be shown that some model is about the phenomena in some real system and not that this fact somehow links the theory to the relevant real system as well. Since in modeltheoretic terms the models of a theory both interpret the theory and conceptualise the aspects of the real system in question, here it is argued that the property of empirical adequacy concretises not only (at least one of) the models of some theory, but also the theory itself. Empinical adequacy of a theory thus does not collapse into a simple notion of "truth" but rather in a more subtle way, it collapses into the notion of "articulated reference respecting the data 
Related to the problems of underdetermination are problems conceming the "extension" of theoretic terms. Sneed (1983: 352) thinks - correctly - that "[t]he meaning of terms refeming to theoretical elements in a theory depends on the theory's empirical claims and may change as the theory develops naturally". Let us see what he (Sneed, 1983: 352, 353) views as the "meaning" of a theoretical term such as the mass function in Newtonian mechanics. First he (ibid.) claims that we have to know the extension of the theoretical terms itself, i.e. the extension of "mass". At first the denotation of the term may be taken (ibid.) in terms of a particular set of ordered pairs sparticle, real numbers (i.e. a function). This will not do, however, according to Sneed (ibid.), since there is no specific absolutely fixed set of mass values that scientists necessarily have to use. (They may, for instance, change the units in which mass is measured.) Then the extension of mass may perhaps be extended such that it includes "all 'acceptable' assignments [of values] in actual applications" (ibid.). But this would in its tum have to be extended to include all as yet "undiscovered" mass assignments. (All of this is basically an example of the underdetermination of "mass" by the theory in question's individual models.)

The problem of the extensions of theoretical terms takes on a different character in model-theoretic terms, because the notion of a scientific theory serves all the while as a kind of touchstone for its various models. In this sense, extensions of theoretical terms are given in terms of the models of a particular theory. The interpretative function of conceptual models replaces the old rigid statement approach notion of "bridge" principles. The point of a modeltheoretic realism is exactly that instead of offering simply one intended model of "reality", a theory is depicted as a way of constructing or specifying a collection of alternative models, each of which represents, explain5, and predicts different aspects of the same (or different) real system(s). Newtonian dynamics offers a theory that is true of some real systems and has many different - very familiar - models - e.g. the harmonic oscillator and a two-body system in which one point mass moves in a closed orbit around the other (strictly speaking both move around their joint centre of mass).

The issues conceming the extensions of theories come down to another related issue, 
namely the "unfixability" of the set of intended applications - the conceptual and there-in embedded empirical models in my terms — of a given theory. Sneed (1983: 353) remarks that certain specialised laws may be added to the "basic" (ibid.) laws in the core of some theory. For instance (ibid.) "[t]he 'theory element' containing [Newton's second and third laws, i.e. the 'basic' laws of Newton's theory] alone determines one extension for 'mass', given the range of intended applications. If we add the requirement that only 'gravitational' forces appear in some subset of the range of intended applications, we impose additional requirements on the entire array of mass functions and thereby change the extension of 'mass' in Newtonian particle mechanics. ... In part, as the specialisation net grows the extension of 'mass' could be narrowed down so much that the mass function for the entire range of intended applications [is] uniquely determined 'up to a change in units'". In modeltheoretic terms also, the class of models can be "narrowed down" by strengthening the axioms of the theory ${ }^{203}$, which are close to the structuralist "basic" or "proper" empirical laws of the theory.

A model-theoretic realism implies that a naive interpretation of incommensurability is a rather empty philosophical notion that has no real beaning on the process of science. Such a model-theoretic account of science makes it possible to examine technically various formal relations between different theories and their interpretations. Examples of such relations are:

- The interpretability of language $L_{1}$ in language $L_{2}-$ in which every primitive term of $L_{1}$ becomes a defined term in $L_{2}$.

- The interpretability of theory $T_{1}$ (in $L_{1}$ ) in theory $T_{2}$ (in $L_{2}$ ) - where $L_{1}$ is interpreted in $L_{2}$ and all the sentences of $T_{1}$ as interpreted in $L_{2}$ become sentences of $T_{2}$.

- Limiting interpretations - where terms of $L_{1}$ become, when some limit is taken, equivalent to terms of $L_{2}$. For instance Einsteinian mass (which depends on velocity) becomes Newtonian mass (which is independent of velocity) in the limit when the velocity is very small compared to the velocity of light.

Aspects like these and many more similar considerations must be taken into account when considering whether two theories are incommensurable or not. Only then could one 
eventually come to a definition (or probably different definitions) of what "incommensurability" may mean, and then continue to treat it technically.

\subsection{Conclusion}

A last few remarks on the scope of a model-theoretic realism such as the one expounded in this chapter. In Chapters 1 and 2 I have mentioned that although I am concentrating on the natural sciences, this kind of model-theoretic account is probably applicable to the social sciences as well. It should be obvious though that different levels of both the formulative and the interpretative chains of such a model of science will be emphasised differently. For instance, the typical sociology of science fixation with analysing the nature of the particular scientific community in question, and the typical constructivist dependence on the theoretical framework in question, both at least result in emphasising the role these factors play in the process of science. At most these schools of thought have shown that sciences more vulnerable to the influence of these factors (sometimes to the exclusion of factors such as empirical testability - think of the interpretation of the fine arts and literary studies) should be treated differently from those who do not have this "weakness".

A model-theoretic realism nicely caters for both these points. It is obvious that the construction of both the conceptual intended and the interpretative models of some theory would be a far more complex enterprise in the social sciences than in the empirical sciences. However, at the same time, a model-theoretic account of science is the one consistent account available I know of that acknowledges and addresses the role of typical socioconstructivist features of the growth of scientific knowledge. Kuhn (1977: 295) points out that if the term "paradigm" is to be fully understood, "scientific communities must first be recognised as having an independent existence". The same goes for understanding the development of scientific theories - from their origin to their applications - in a modeltheoretic context.

lan Hacking (in Tauber, 1997: 163) sets out two types of realism, i.e. realism about theories, and realism about entities. He (ibid.) describes realism about theories as a result 
of our desire to ".., form true theories about the world, about the inner construction of matter and about the outer reaches of space", while he (ibid.) claims that realism about entities "... asserts the existence of at least some of the entities that are the stock in trade of physics", and he (ibid.) understands by "entities" for instance "processes, states, waves, currents, interactions, fields, black holes". A model-theoretic realist is mostly a realist about entities, with the understanding that these entities are described (and their behaviour explained) by scientific theories and their conceptual and empirical models.

Fine (1986a: 150) claims that the process of science has a teleological side in the sense that "... the significance that realism attaches to science lends itself to the view that what science does ... is exactly what it aims to do. Thus we get the realist slogan that science aims at the truth, with the realist connection between truth and the Wortd being understood". In model-theoretic terms the aim of science simply is to offer certain idealised "insights" into the complex workings of "Nature", No statements about "absolute truth" or the unqualified "truth of scientific theories ${ }^{\mathrm{x}}$ are offered in such an approach and it is shown that such notions are empty concoctions left over from the philosophy of science practised during the first half of the twentieth century. Rather, systems in reality may be explained in terms of certain models interpreting a certain scientific theory. Scientific theories cannot be universally true, but merely true in (a) particular conceptual model(s) of it.

Moreover, these theories' statements are never meant to apply universally in a ceteris panibus way. Scientific theories are formulated ceteris paribus (see my discussion in Chapter 4), however, their application and interpretation are context-specific, i.e. model-specific, and never fixed in any unqualified way. The closer we get to systems in reality, the fewer of these ceteris paribus conditions we need. Models are idealisations of real systems. However these ideal circumstances in which the theory is shown to be true are not as they are by virtue of the fact that all other things remain equal, but are rather the results of focussing on a specific real system and constructing a particular model driven by the scientific tradition, application goal, and other "thematic" factors present.

It is moreover precisely because of the idealised nature of models that we still have the 
possibility of having contact with reality given the abstracting way in which science and its enterprises operate. Idealisation does not mean universalisation, however. It is precisely because the model is so specific - in terms of its focus on selected features of some real system - that it is so ideal. This however in no way implies that no links with the "real complex" systems are possible, but rather that these links are established and checked in a certain specific (scientific) way, i.e. a model-theoretic way.

The slogan of a model-theoretic realism is "truth without universality". This is meant in the sense that it is the specific model-theoretic kind of truth that is at issue, and that theories are never examined for their relevance to reality in their stark linguistic terms, but always in terms of their (conceptual) interpretations in their various models. Theories in this sense are not viewed merely as general knowledge propositions, but rather as the means of organising systems of their models in such a way that certain systems in reality can be (empinically) "embedded" into these models.

A last remark on the nature of scientific reality. Trivially, for those who assume that science is about independent "Nature", we all address the "same reality". However, the notion of a particular "scientific reality" - that I have claimed above to be constructed and to offer "snapshots" of "Nature" - can also be communicated and shared although it may well be that no objective (neutral) way exists to describe it, precisely because of its model-theoretic origin and its changing historical context. 


\section{CHAPTER SIX: CONCLUSION}

\subsection{The meaning of a model-theoretic realism for philosophy of science}

A model-theoretic approach to science supersedes and encompasses the best aspects of both the statement and non-statement accounts of scientific knowledge. Although in both the latter accounts - albeit in different ways - it seems that the notion of a scientific "theory" - however this notion is interpreted - may be given some realist interpretation at least, the unnatural (and simply wrong) rigidity of the statement approach's correspondence rules as well as the non-statement disconnectedness of scientific theories and their models do not allow for reference to reality in a satisfactory way. In a model-theoretic approach a scientific theory is a certain (deductively closed) set of sentences linguistically expressed. ${ }^{204}$ The conceptual embodiments of the contents of these theories are done via their models and the referential relations in question in a realist context are determined both by the empirical substructure, isomorphically embedded in some conceptual model of the relevant theory, and by the nature of the real system in question. By maintaining such an encompassing interpretational link from the theories themselves all the way through their models to some real system(s) the complicated and changing character of science may be described and accounted for in a more adequate way than is perhaps the case with some statement and non-statement approaches to science.

The problem haunting philosophers dabbling in more metaphysical aspects of scientific theories - like Bhaskar and the later Cartwright - is exactly to show that - and how through the complicated contingencies of science a given scientist is still dealing with the same physical phenomenon as her predecessors and her peers. These considerations are related to the underdetermination issue, since the problem is to show that the scientist in question can "get to" the same phenomenon whichever theory (from the class of theories underdetermined by the phenomenon in question) she chooses to use. And, in this sense, Cartwright's capacities - in as far as they somehow have some stabilising influence on the "complex uncontrollability" of nature - may indeed seem to have a lot going for them. She (Cartwright in Boyd et al., 1991: 386) writes: "Competing theoretical treatments - treatments 
that write down different laws for the same phenomena - are encouraged in physics, but only a single causal story is allowed. Although philosophers generally believe in laws and deny causes, explanatory practice in physics is just the reverse". First, we know that science is not about stabilising in the sense of somehow changing the complexity of Nature into a controlled system. Rather science is about offering a glimpse as it were of some specific aspect of Nature. Such a representation of Nature is perhaps "stable" in so far that it focuses only on relevant features of the aspect of nature it concentrates on at a given time. However a model-theoretic realism shows that underdetermination - in a sense the converse of allowing only a "single causal story" - is a necessary characteristic of science, since the abstracting nature of the methodology of science specifically implies that other routes to the same conclusions are possible under a different abstraction from the same aspect of nature.

The necessity of looking to Nature - and, in philosophy of science terms, thus perhaps turning to metaphysics - for solutions to the supposed puzzles conceming underdetermination dissolves within the framework of a model-theoretic realism. As pointed out in Chapter 5, the main assumption of such a realism concerning Nature is simply that it (i.e. Nature) exists independently of science. This basic condition is emphasised and worked out by the model-theoretic insistence on the roles that both science - in the guise of a specific conceptual model of a given theory having isomorphically embedded into it a certain empirical model - and Nature - in the sense of the characteristics of some real system satisfying the empirical results embodied by the specific empirical model in question - play in the processes of science. No metaphysical charactenstic of Nature somehow worked into the mechanics of science is necessary to make sense of a scientifically realist picture of Nature. Rather the definition of the methodology and strategies - and aim - of science, model-theoretically interpreted, already takes care of all of that. And, a scientist can "know" that she is working with the "same phenomenon", even if using "different" theories, simply because of the possibility of analyses that a model-theoretic realism offers of the different empinical links between different empincal models of different conceptual models in (perhaps) different theories. Delailed analyses of these empirical links will reveal common factors on 
the reality side of the link (e.g. light blobs observed through different telescopes by different people at different times indicating - by careful analyses - a common factor called "Neptune") which entails the "same phenomenon". There is, however, no universal prescription for these analyses.

Closely related to this is Putnam's so-called model-theoretic "paradox" (see Putnam, $1978)^{205}$. Van Fraassen (1997: 18) points out that if the criteria narrowing down the relevant interpretation of the language in question are "solely internal" (ibid.) such that "certain sentences must come out true (since they reflect our intensions about how to use the language) (ibid.), then, yes, it seems as if indeed practically any theory is true. Modeltheoretically speaking it is part of the basic conditions of science (here in contrast to mathematics and logic) that these criteria are never solely internal. A "successful" mapping between the individuals of an empirical model embedded into a conceptual model of a given theory and the entities and relations of some real systern depends just as heavily on the nature of the real system as it does on the logical relations of satisfaction between the theory, its conceptual model(s), and its empirical model(s). Van Fraassen (1997: 20) points out the trivial fact that not only does one such a mapping consist in Putnam's ideal case (see Endnote \#205 above), but indeed obviously many such mappings exist. The actual question thus is: how do we know which one to pick? Well, again, we do not do anything as trivial as merely "picking". A "successful" mapping is determined both by our scientific activities that gel finally into a certain empirical model of a given theory, and by the real system of which the empincal model in question is offening a certain (scientific) image. That is why the notion of scientific truth model-theoretically interpreted is a referential notion without any necessary metaphysical content.

Turning to a related issue, Van Fraassen (1997: 36$)$ explains the problem concerning the "fixing" of the reference of language terms as follows: "... each of our predicates has an extension, and might have had a different extension. But unless they have the right extension, we can't use our language to frame genuine, non-trivial empirical statements or theories. So, under what conditions do they have, or acquire, the right extensions?" (ibid.). Later on he 
(ibid.:37) comments that we obviously do not do any kind of fixing and whether Nature does any kind of "glueing" is not really a sensible question — in any absolute sense I might add. This leads us to the historical note in the next section of this chapter that concerns the nominalist-realist divide regarding the nature of reference, and so the very definition of language terms (including so-called "universal terms"). Model-theoretically speaking, language has to be interpreted nominalistically so that science can be interpreted realistically. Only if we can handle the fact that there are no fixed one-to-one unique mappings between universal language terms and objects in reality, do we have a chance of making sense of underdetermination and the unfixability of Nature. Paradoxical as this may sound, this is the only non-metaphysical way to be a scientific realist. The suppleness of model-theoretic realism is the only answer to the seeming chaos of underdetermination - and also of "overdetermination", in the trivial sense of "Blue" not denoting only "truly blue" (whatever that may mean) objects.

Promising research areas in philosophy of science that successfully use model-theory and its interpretation of science are for instance the work being done in the area of verisimilitude, and analyses of the entire experimental enterprise of science - the empinical aspect of science is extremely underrepresented in philosophy of science and a modeltheoretic account of science offers a challenge to philosophers to rectify this. ${ }^{206}$

\subsection{Historical note}

Traditionally there are basically two views of language and its terms: norninalism and realism. Typically nominalists deny the existence of universals. That is they do not support the existence of the referents of general terms such as "flower", or "blue". They claim the resemblances between particular entities are sufficient to justify application of the same general term to all of such entities, and thus deny the need to appeal to any other (universal) entity to classify individual things. Realists traditionally have protested that such an approach still implies at least a tacit implication of reliance on universals since the act of classification of things common to a certain set of individual entities implies a resemblance in some general 
respect. In other words realism with regard to particulars rests on some kind of realism about at least some universals, namely those that are the reputed referents of bona fide natural kind terms. More recently, nominalism usually implies the refusal to acknowledge the existence of abstract entities in general, whether they be particular or universal. Nominalists thus have a rather less rigid approach than the typical (naive) realist attitude to the problem of reference in so far as they claim that general terms - i.e. "universals" - may exist only for the purpose of language and need not "correspond" to "real" entities at all.

Before we discuss the model-theoretic position with regard to these issues, let us briefly examine the notion of an abstract entity or "universal" a little closer. The usual Platonic realist interpretation of these terms concludes that abstract entities have necessary existence, given that they have no causes or effects and no spatio-temporal location. Traditionally universals are taken to be the supposed referents of general terms like "flower" and "blue" and as such are thought to be entities distinct from any of the particular things describable by these terms. Platonists therefore claim that there has to be something by virtue of which all things describable as blue are indeed blue. Aristotelian thoughts on this issue however come down to almost opposite claims: the universal "blue" exists only inseparably from the existence of particular blue things. According to Aristotle only particulars can be genuine subjects and so universals can only be predicates of subjects - and obviously then never be subjects of predication themselves. In the Platonist scheme of things though, it is entirely possible to have predications with universals as subjects. Aristotle claimed the universal to be grasped by the mental process of abstraction ${ }^{207}$, while Plato thought the existence of universals to be completely mind-independent.

In the twentieth century philosophers such as Russell and the early Wittgenstein continued in the more conservative realist tradition which seems to subscribe to the belief that there is only one language and only one reality and that these two should only be linked in one-to-one relations. ${ }^{208}$ Quine and Kripke also seem to have been fighting mainly on this side of the battle. The notion of a so-called "rigid" designator for instance supposedly captures the idea that throughout all possible worlds there are words that refer to the same individual. The 
relations can vary but never the basic entities. It is necessary that certain things are named in certain ways (or by certain terms). ${ }^{209} \mathrm{I}$ think it probable that somehow underlying all such approaches is the belief in some notion of absolute truth such that it is not allowed to arbitrarily ascribe "reality" to general terms. Nancy Cartwright's work on the lying laws of physics offers an interesting - albeit perhaps not a "typical" - case in point. The need to claim that these laws "lie" seems to me to be the direct result of some kind of notion concerning absolute truth. Why else should it matter that these laws can be concretised in so many different ways? $?^{210}$

During the last three or four decades there have been two kinds of reaction against the typical one-world-one-language account of the nature of abstract entities. The first radical reaction is offered to us by the so-called "postmodem" notions of language and world that, in their extreme forms, seem to imply that Rorty's smashing of the "mirror of nature" eliminated all the chances - if there ever were any - to be able to describe reality - as "Nature" - at all. A more rational, rather than radical, reaction to the strict realist account of the reference of general terms is offered by a model-theoretic realism. The defenders of such a realism try to get some kind of a grip on the many-to-many relations between language and the models in which the sentences of the relevant language are true, and show that "truth" should be interpreted referentially in model-theoretic terms and never in terms of an infinite cancelling or postponing of meaning.

Within such an approach it is neither assumed - as in the case of defenders of formalism perhaps - that language expressions are merely "black marks" on white paper or air vibrations that can mean absolutely anything, nor is it claimed that abstract terms have any pre-determined reference or meaning. A model-theoretic realism escapes the extreme nominalist features of an approach such as that offered by so-called formalists because in model-theoretic terms not merely syntactical factors ${ }^{211}$ are at issue, but also - most importantly - issues of semantics come into play. Tarski, Montague, and Adams may be viewed as some of the pioneers of this stream in philosophical thought with regard to the nature of abstract terms. There is however among model-theoretic advocates also some 
sympathy for the Platonic ideals. Perhaps Gödel offers the best example of Platonism in the philosophy of mathematics while physicists such as Stephen Hawking may also be a bit tainted in this respect. In these - more Platonic - circles the mathematical tools most used are those of set theory and number theory. ${ }^{212}$ Model-theoretic realists however give more attention to abstract algebras ${ }^{713}$ - concemed with notions such as nings, groups, and vector spaces. These notions allow one to work with terms that do not necessarily always refer to the same things.

This is the basis of model-theoretic realism: the same language (theoretical) terms can refer to more than one entity in some model of the language (theory), and also the same object - or range of objects - in some real system can be given reference by more than one model. As mentioned above, it is these referential relations between language terms and the terms in the model of the language in question that may solve the problems concerning the referential relations of Quine and Duhem's underdetermination.

Jaakko Hintikka (1989: 53) characterises the traditional nominalist-realist divide in terms of language as calculus and language as (the) universal medium. He (ibid.) explains the latter notion as essentially an attempt to "... escape our language and ... look at it and its logic from the outside". This renders a model-theoretic realism "impossible" (ibid.) since the semantics - because of its supposed absolute character - of the relevant language becomes "inexpressible". From the more nominalist, "language as calculus", point of view however, the semantics of the relevant language becomes expressible to such a high degree that the notion of its interpretation as absolute becomes completely untenable. Hintikka (1989: 54) writes: "The term 'language as calculus' is not calculated to indicate that on this view language would be a meaningless jeu de caracteres - that is not the idea at all. Rather the operative word highlights the thesis that language is freely re-interpretable, like a calculus". This, essentially, is what the notion of "epistemological relativism" 214 in in my account is meant to imply too.

Hintikka (1989: 55) continues to point out that the re-interpretability of our language, according to the "language as calculus" view, implies that we can "... chose freely also the 
'universe of discourse' as it [the relevant language terms] is designed to apply to" (ibid.). This is very much in accordance with the fact that a model-theoretic realism claims to have the ability only to show that some theoretical terms are "about" certain aspects - systems - in reality. Given the abstractive selective nature of science - as discussed above in Chapters 2 and 5 - and given this "free" choice of domain of interpretation ${ }^{215}$, it seems obvious that a model-theoretic realism of science is at least very promising.

However, again, as Hintikka points out too (see above quote), this "choice" is not free to any absurd extent, In philosophy of science terms the "restraining" factor lies in the fact that a theory's terms - or some of them at least - may be shown to refer to some entities and objects of some real system not only because of the nature of the specific model interpreting the relevant theoretical terms at a certain time. Such an interpretation offers the necessary basic platform from which theoretical reference can be determined, but is by no means the final word. The final word is given by empirical data which constitute some empinical substructure of the relevant model on the one hand, and which represents a conceptualisation of features manifested by real interactions with the relevant real system on the other hand,

Thus, it is in a sense both the "way" in which the world "is" (conceptualised via the specific empinical model in question) and the theoretical - abstract - terms of the theory (interpreted via the specific conceptual model in question) that determine any articulation of possible reference. A model-theoretic realism thus displays a referential rather than a correspondence attitude towards the real systems science is "about". And, since the basic assumption of a model-theoretic realism is that science is about (human-independent) "Nature" via the descriptions and explanations of certain real systems offered by science's idealised models of the theory in question, any accusations of "rigging" the models of a theory such that the theory "really" refers - or amything akin to such claims — are rather absurd.

In conclusion, the Aristotelian orientation of a model-theoretic realism can finally be given its nightful recognition. According to a model-theoretic realism such as mine, the general exists only in the particular. The meaning of the general and the particular are inseparably 
entwined. My criticism against the non-statement approach is based on this notion of the nature of general terms, since merely "giving" the theory "in terms of" its mathematical structures leaves out any real interpretation of the nature and role of general terms in the interpretation of science. This might be viewed - and I am sure it is in certain circles - as a rather clever method of escaping the debates remarked on above. However it is my conviction that science cannot be made to be meaningful without these terms.

The essential link between the general terms of scientific theories and their interpretation in the various models of the theory regulates the rest of the referential process. In this sense, I prefer not to speak of the "universal" terms of a theory since these terms never have absolute interpretations. The terms of a theory are "general" in the sense that they are the result of certain abstractive manipulations of the object of scientific investigation at a certain time. Their (particular) meaning can be "given back" only by interpreting them in the limited context of the various conceptual models of their theory and, finally, by finding an isomorphic relation between a substructure of the conceptual model in question and some empinical conceptualisation (model) of relevant experimental data. In this sense the notion of scientific "truth" becomes inextricably linked with that of reference, as it - given its model-dependent nature - should be. 


\section{ENDNOTES}

1. Hilary Putnam's term.

2. See Camap (1958).

3. Closely related to the structuralist approach is the approach of Ludwig (1990), which I shall not analyse here, but which is worth mentioning, since it is clearly important, although it does not receive much attention in the English literature on philosophy of science.

4. Przelewski (1969) analyses the structure of scientific theories in terms of theories formulated in first-order predicate logic. He wants to develop an empinical semantics for such theories, and thus needs to offer some analysis of the empinical interpretation of the basic predicates of scientific theories. He offers this analysis in model-theoretic terms: "in part, an interpretation of a given language is identified with a model theoretic entity - a model $M$ of language $L$. $M$ assigns to each non-logical constant of $L$ a suitable settheoretic entity as its denotation. Thus, e.g., a one-place predicate of $L$ is interpreted by $M$ as denoting a certain set of objects from the universe of $L$. ... my ultimate aim [in (1969)] has been to answer the question ... how is an empirical interpretation possible ..." (Przelewski, 1974:401, 402). Tuomela (1972b, 1974) goes to great lengths to point out the problems involved in Przelewski's assumptions concerning the fixing of the universe of the language $L$ in advance - which Przelewski claims is necessary to do in order to "explain the fact of empirical interpretations" (Przelewski, 1974:404).

5. See Przelewski and Wójcicki, 1969.

6. Pearce and Rantala (1983) claim that they offer a view in which theories are "abstract systems" free of any explicit logical interpretation. This is very interesting, especially since it seems to allow for problems conceming theories too complex to reconstruct in elementary terms, and also since it makes it possible to allow the choice of logic to be an extra-logical (maybe philosophical?) issue.

7. Note that in both the statement and non-statement approach the rational reconstruction of a scientific theory is given in terms of an uninterpreted language, which implies, in principle, the possibility of an unlimited number of interpretations of the language. However, in both cases the possible interpretations of the uninterpreted language are limited at least in the following senses. In the statement approach any interpretation must satisfy the axioms of the theory, and in the non-statement approach an interpretation must belong to the described class of structures.

8. According to Tarski (1956) a model of theory $T$ is a possible realisation in which all valid sentences of the theory $T$ are satisfied.

9. "To define formally a model as a set-theoretical entity which is a certain kind of ordered tuple consisting of a set of objects and relations and operations on these objects is not to rule out the physical model of the kind which is appealing to physicists, for the physical model may be simply taken to define the set of objects in the set-theoretical model" (Suppes, 1960: 290, 291). instead of "define" it would be preferable to say "be in one-toone correspondence with". Mathematical sets of non-mathematical objects are problematic. See my comments on this issue in Chapter 3.

10. I shall from now on sometimes speak of Newton's "theory" when referning to his laws of motion and his law of gravitation.

11. See also Hesse, M. 1963. Models and analogies in science. As Redhead (1980, 149) remarks, Hesse does however point to the role of mathematical models in the 
development of theories, although she pays much more attention to the role of models in science in terms of analogies.

12. I am not implying here of course that these models are "necessarily true" depictions of systems in reality, but simply wish to point out that here at least the possibility of such a turn of events is possible, albeit by very complex ways. I shall come back often to this point.

13. An example that nicely illustrates the relation between Hesse's analogous models and so-called iconic models is given in Da Costa \& French, 1990: 250; They write "In a nucleus ... there are too few particles for a statistical treatment, and there is no overriding centre of force which would enable us to treat the forces between nucleus as small perturbations. For this reason, physicists have fallen back on the 'as if' methods of attack, also known ... as the method of nuclear models. This method consists of looking around for a physical system, the 'model', with which we are familiar and which in some of its properties resembles the nucleus. The physics of the models are then investigated and it is hoped that any properties discovered will also be properties of the nucleus. ... In this way the nucleus has been treated 'as if' it were a gas, a liquid drop, an atom, and several other things".

14. The arguments that $\mathrm{Da}$ Costa and French (1990: 260) offer in support of their claim that models can only be false do not have anything to do with mathematical models, but only with the use of models as iconic models. Therefore they can only be allowed to conclude that iconic models are false, which in the first place is rather obvious, given the analogous "as if" role of these models, and in the second place this kind of remark is not really very important in the context of surveying the model-theoretic (in terms of mathematical models) interpretation of science.

15. Redhead (1980: 147) remarks that models are used as "impoverished theories" if a theory is so complicated that it is very difficult to draw any kind of empirical conclusion from it, since comparisons between the theory and experimental results prove to be too complex. He also shows clearly that the role of these kind of models is not to be confused with the role a Tarskian model plays in the process of science: - "... the important ingredient ... [is] that [the model] and [the theory] logically contradict each other, so that we believe [the model] to be false insofar we believe [the theory] to be truen (Redhead, 1980: 147).

16. Note that also theories which are proved somehow empirically inadequate through experimental or some other type of empirical investigation, tum into "impoverished theories" - for example (Redhead, 1980: 147) Maxwell's kinetic theory of gases is now known as the billiard ball model of gases.

17. See Ruttkamp, $1997 a$, as well as Chapter 2 of this thesis.

18. In my scheme of things, only the "intended" model of some theory has the potential to develop into a "full-fledged theory". See my explanation and discussion of these notions in Chapter 2.

19. See Suppes's example of Mach's research in Suppes, 1960: 296.

20. See Van Fraassen, 1980, pp.45ff., and Chapter 5 of this thesis.

21. Suppes and Giere and Wójcicki all seem to think that scale models and - even more physical perhaps - models of aeroplanes and cars are at least part of the notion of a "physical" model. In my terms part of the conceptualising that culminates in the intended model may well be directed towards such a type of model - or not, depending on the particular line of research in question. (See Chapter 2.) 
22. Note that "physical" here does not necessarily mean concretely physical, but merely serves to show the more "direct" link with the real system of reality being examined. (See endnote \#21 as well.) Any activity ending in the construction of a model is conceptual in the sense that various activities of abstraction and even idealisation are performed. As far as the very few times that an actual concrete model is built go - think for example of the model that Watson and Crick (see Giere, 1991) built of the DNA molecular structure - I would say that, usually, at the same time some kind of conceptual model is also created.

23. Hausman's (1992, chapter 4) approach seems to bear me out here - he (Hausman, 1992: 81) remarks for instance that "[e]ven though models in economics need not be as abstract as those which characterise mainstream theorising, they will never apply to economic reality cleanly. Insofar as one has hopes for economic theory at all, there will always be some need to divorce conceptual development and empirical application. 'Unrealistic' model-making is unavoidable for theoretically inclined economists".

24. This is supported by Harrod (in Wolfe, 1968: 189) as well, as he concludes his article by acknowledging that he would prefer that in science (including social science) "... the word 'model' should be confined to formulae relating to posited entities, viz. to entities that we can never directly observe and about the very existence of which we cannot be sure". And he (in Wolfe, 1968: 190) goes on to say that "[i]f we want to bring 'models' into economics, but to keep them meaningful, we might confine the term to a system of equations, not all of which are tautologies. Some at least might have adjustable parameters. It might be made a condition for the use of the word that some equations explicitly omit to take account of fringe influences".

25. Some sections of this chapter will be published as Ruttkamp (1999a).

26. Although not as common-sensical as Fine's (1986b) "natural ontological attitude" perhaps.

27. One may ask how - and even if - it is possible to distinguish between conceptual and linguistic levels without giving a clear and valid answer to the question of whether it is possible to think without language. I am however not making rigid distinctions here. What I am doing, in fact, is to depict the development of scientific research by emphasising one by one the real, conceptual and linguistic aspects of this developmental process. And, moreover, I am claiming that there always is interplay between these aspects.

28. See the conclusion to Chapter 5 for a little more on this issue.

29. Remember that set theory and hence (most of) mathematics can be formulated in firstorder predicate logic.

30. In a language such as $L$, we usually have the following eight categories of basic symbols available:

- a countable infinite set $\left\{v_{i}\right\}$ of individual variables

- a (possibly empty) set of individual constants

- a nonempty set $\left\{P_{\alpha}\right\}$ of predicate letters, and, associated with each predicate letter $P_{\alpha}$, there is a positive integer $\delta(\alpha)$ called the arity of $P_{\alpha}$, which gives the number of individual variables which are predicated by $P_{\alpha}$

- a (possibly empty) set of function symbols

- the equality symbol "="

- logical connectives (details not important for my purposes here)

- quantifier symbols (ditto)

- punctuation symbols (ditto). 
31. A mathematical structure $U=\left\langle A,\left\{R_{\alpha}\right\}\right\rangle$ consists of a set $A$, the domain of $U$, and a set of relations $R_{a}$ (one for each $\alpha$ from some index set) defined on domain $A$. The sets $A$ and $\left\{R_{\alpha}\right\}$ both may be infinite. $A$ relation $R_{a}$ on domain $A$ is defined as a set of ordered $\mu(\alpha)$-tuples of elements from domain $A$, where $\mu(\alpha)$ is a unique non-negative integer associated with the relation $R_{a}$.

32. In other words the mathematical structure $U$ will count as an interpretation of the language $L$ if and only if the arity of the relations $R_{\alpha}$ correspond to the arity of the predicate letters $P_{\alpha}$. (That is, if $\delta(\alpha)=\mu(\alpha)$.) In this case $U$ is called a realisation of the language $L$ (and we can say that $L$ is appropriate for the structure $U$ ). We call the relation $R_{\alpha}$ the value of $P_{\alpha}$ in the realisation $U$ of language $L$. (If $L$ has constant and function symbols, they are interpreted as elements of $A$ and functions - of the proper anties on $\mathrm{A}$.)

33. E.g., consider the formula $P x y$. If $P$ is interpreted as the relation $<$ and if $x$ and $y$ are given the values of 3 and 5 respectively, then we say that Pxy is true under that interpretation and we say that formula Pxy in language $L$ is satisfied by the valuation in the domain of interpretation $U$, ascribing the given values to variables $x$ and $y$. (Because 3 is indeed smaller than 5.)

34. Note that a realisation of language $L$ is in principle a realisation of all the sentences in $L$, and this implies that every sentence in $L$ is either true or false in that particular realisation.

35. Einstein referred to these convictions as "free conventions" (Holton, 1995: 464). "These themata, to which [he] was obstinately devoted, explain why he would continue his work in a given direction even when tests against experience were difficult or unavailable (as in General Theory of Relativity), or, conversely, why he refused to accept theories well supported by phenomena, but, as in the case of Bohr's quantum mechanics, based on presuppositions opposite to his own, ..." (ibid.: 457).

36. Wójcicki (1994:142) speaks of a "factual interpretation" of a theory that is determined by the "relevant world view", or the relevant paradigm, research programme, or research tradition. He says: "One cannot understand an empirical theory and thus one can know neither what the theory is about nor how to form a theoretical model [my "intended" model] for specific problems relevant to the theory unless one has some idea what is the part (or aspect) of the world to which the theory refers, how this part is related to the others, which is the ontology of all these parts, and how both the claims of the theory and the empirical data on which it is based are related to the entities whose existence the ontology presupposes". These factors are exactly the kind of factors that, in my terms, influence the construction of "intended" models, and that come into play again when the models specifically constructed with an eye on interpreting or applying the theory are created.

37. Chalmers $(1993,202)$ gives another example of these events: "We may abstract the falling of [a] ... leaf from other aspects of its motion ... We theri apply the appropriate fundamental laws [axioms of mostly "background" theories] to [this model] that [is] the result of our abstraction. We apply Newton's laws to the leaf as a mass subject to the gravitational attraction of the earth only, and denive the law of fall from it. Of course, since we have abstracted from winds, air resistance and the like, our model will not in general serve to describe the fall of any particular leaf. After all, the model is an abstraction. Nevertheless, provided we understand the leaf to have a capacity to fall, governed by Newton's laws, the theoretical treatment via the abstract model does explain the falling of the leaf, as distinct from its fluttering in the breeze". This is a point about which Nancy Cartwright has serious reservations, but with which I am in full agreement. See Chapter 4 for more on Cartwright. 
38. Einstein referred to the movement from conceptual structures or models to theories as a "creative leap" and in this sense referred to theories as "free craations of the human mind".

39. Kepler's laws:

- First law: All planets follow elliptical orbits (and nol circular ones, as Copernicus believed) with the sun situated in one of the foci of the ellipse.

- Second law: The line connecting the sun and a planet sweeps over equal areas of the planetary orbit in equal intervals of time.

- Third law: The squares of the periods of revolution of different planets around the sun stand in the same ratio (i.e. is proportional to) the cubes of their mean distances from the sun.

Newton's three laws of motion:

- Every body continues in its state of rest, or of uniform motion in a straight line, unless it is compelled to change that state by forces impressed on it.

- Change of motion is proportional to the force impressed, and is made in the direction of the straight line in which the force is impressed,

- The forces two bodies exert on each other are always equal and opposite in direction.

His law of gravitation:

- All material bodies attract each other with a force directly proportional to their masses and inversely proportional to the square of the distance between them.

40. This is close to Cartwright's notions of the role of scientific explanation and description. See Chapter 5.

41. See the examples of the discoveries of Neptune and Pluto, as well as other applications of Newton's theory in the following section.

42. Another type of approach to the interpretation of language terms is offered by Hans Lenk's methodological or schema interpretationism. See for instance Lenk (1993), (1995),

43. Whenever I speak of models of theories, I am referring to the notion of model in the Tarskian sense that a model of a theory is an interpretation of the theory under which the set of sentences comprising the theory is true. As mentioned in the previous section, at the start of theory formulation the intended "model" scientists work with is not (initially) such a mathematical model, although at the stage of theory interpretation it becomes obvious that such (intended) models can be easily adapted such that they also are elements of the set of all (mathematical) models of the theory in question.

44. These terms are the terms traditionally referred to as "theoretical" terms. Note that therefore I do not follow in the footsteps of advocates of the traditional version of the statement approach, in the sense that I do not need the kind of inadequate and much too simple distinction they make between theoretical and observational terms in the language of the theory. Rather than this forced division, I propose an approach in which theoretical and observational terms, as well as the difficult "correspondence rules" or "bridge principles" supposedly acting between these kinds of terms, all have natural interrelated and co-dependent roles to play at vanious levels of the scientific process.

45. I claim that Giere's theoretical models, Wójcicki's theoretical and semantic models, and Suppes's physical and set-theoretic models are all mathematical models in this sense. Some of these authors make a similar kind of distinction that I make between these models as "intended" models - Wójcicki's theoretical models and Suppes's physical models - and these models as "conceptual models" interpreting the theory - Giere's theoretical models, Wojcicki's semantic models, and Suppes's set-theoretic models; although not all of them seem to view all these notions as mathematical structures in the Tarskian sense. See Chapter 3. 
46. Kuhn (1977: 301, 302) also refers to the two conceptual movements needed to conceptually move from a theory to some real system - i.e. from the theory to a conceptual model of the theory, and then from that model, to an empinical submodel of it - albeit in slightly different terms, and even if he claims that these two "questions" are usually answered "together" in scientific practice. He (ibid.) asks: "How do scientists attach symbolic expressions to nature?", and then he (ibid.) writes: "That is, in fact, two questions in one, for it may be asked either about a special symbolic generalisation designed for a perticular experimental situation or about a singular symbolic consequence of that generalisation deduced for comparison with experiment".

47. And neither can scientists. The best they can do is to react to unwanted models allowed by the theory by refining the theory's set of axioms in such a way that these models become impossible. But, obviously this is a very difficult task, especially in the first stages of the theory's formulation. And, moreover, trying to define these assumptions too finely, could in principle cancel the possibility of refining the theory in a positive way, i.e. in becoming aware of shortcomings or even errors in the formulation of the theory, via different models of it, offered by other interested scientists. Einstein, for instance, had a static universe as his intended model for his general theory of relativity. It so happened, however, that other physicists constructed models in which the universe is anything but static. Then Einstein, initially rather upset, changed his original set of axioms in order to prevent the possibility of constructing such models. (Afterwards he conceded that he made a big mistake because of the implications of the expansion of the universe and the "Big Bang" model.)

48. "... it is ahways legitimate for scientists to ask and sometimes possible for them to answer, questions about whether gasses are really composed of molecules or whether the earth really moves. Such questions cannot be rephrased as questions about the plausibility of our conceptions" (Bhaskar, 1978:155). Well, the verification of our conceptual models depends on being able to show how experiments concerning the data in question may be linked to these models (via certain empirical models). However, what Bhaskar means, I think, is rather that science does not determine the structure of reality, but rather discovers it.

49. Obviously realist issues and the nature of scientific progress are related. I shall here refer to the model-theoretic realism I advocate only when necessary to illustrate or support my claims about the cumulative nature of scientific knowledge. The issue of model-theoretic realism will be discussed in Chapter 5 .

50. See Heidema, J. \& H.J. Schutte (1978).

51. See Einstein, 1956: 11ff., 65ff.

52. Where i equals $\sqrt{-1}$.

53. See also other non-statement approach advocates, like Suppes (1967, 1989), Wójcicki $(1979,1994)$ and Van Fraassen (1980), discussed in Chapter 3.

54. Think, for example, of students able to cite all the rules (or laws) of a specific area of their subject matter, who are still unable to apply this knowledge in any concrete way.

55. In section $X$ of The structure ..., Kuhn (1970: 126) asks: "But is sensory experience fixed and neutral? Are theories simply man-made interpretations of given data?". And he answered: "... Yes! In the absence of a developed alternative, I find it impossible to relinquish entirely that viewpoint. Yet it no longer functions effectively, and the attempts to make it do so through the introduction of a neutral language of observations [Quine] now seem to me hopeless". In the Postscript, Kuhn (1970: 193) tries to solve his problem by drawing clear distinctions between sensory "stimuli" and "sensations" or "perceptions". 
He (ibid.) writes: "Notice now that two groups, the members of which have systematically different sensations on receipt of the same stimuli, do in some sense live in different worlds. We posit the existence of stimuli to explain our perceptions of the world, and we posit their immutability to avoid both individual and social solipsism. About neither posit have I the slightest reservation. But our world is populated in the first instance not by stimuli but by the objects of our sensations, and these need not be the same, individual to individual or group to group".

And, he continues to say that it is because we have been conditioned to see a one-to-one mapping between stimuli and sensations, that we have such difficulty in recognising that the two viewers actually see different things. In reality, we should - and do - know that the same stimulus may produce very different sensations and that very different stimuli can produce the same sensations.

56. See his article in Tauber, 1997.

57. Kuhn describes (Tauber, 1997: 233) the meaning of these terms as "part of what one must have in the head to use the word properly".

58. See Chapter 5 for more on these issues.

59. See Cook, A. 1994. The observational foundations of physics. Cambridge University Press.

60. Newton "did not produce mere mathematical constructs or abstractions that were devoid of any content of reality other than 'saving the phenomena', but he did create what he conceived to be purely mathematical counterparts of simplified and idealised physical situations that could later be brought into relation with the conditions of reality as revealed by experiment and observation" (Sarlemijn \& Sparnaay, 1989: 6). He also preferred synthetic geometry to Descartes's analytical geometry and even to his own calculus, because both the latter have levels of proof without any clear physical interpretation. Bishop Berkeley even referred to the infinitesimals in Newton's calculus as "the ghosts of departed quantities".

61. Newion affirmed Aristotle's inductive-deductive method - he called it the "method of analysis and synthesis". Newton declared that "although the arguing from experiments and observations by induction be no demonstration of general conclusions, yet it is the best way of arguing which the nature of things admits of (Newton, 1952: 404).

62. "The correspondence between atomic structure and the solar system was thought to be self-evident" (Sarlemijn \& Spamaay, 1989: 7).

63. Large sections from this chapter will be published in Ruttkamp (1999a).

64. See Suppes $(1954: 244)$.

65. Obviously the axiomatisation of theories in a set-theoretical framework does not necessarily imply a non-statement approach - it is quite possible to stick to the statement approach and define a set of valid sentences corresponding to the settheoretic predicate by making use of the same axioms used to define the set-theoretic predicate in the first place. This would be closer to the model-theoretic approach spelled out in Chapter 2.

66. Adams (1959) discusses the reduction of one paradigm of science to another, and so touches on some related questions of underdetermination as well.

67. Suppes (in Wójcicki in Humphreys, 1994:148,149) writes: "The more I think about scientific practice and reflect on how to give an accurate account of the complicated 
processes that go into experimentation, the more I am persuaded that there are a large number of distinctions needed to describe experimentation thoroughly, especially as data are purified for quantitative, and even more statistical, analysis. It is a long way from running around the laboratory doing one thing and then another, to having a set of data as printout or on a computer screen ready for analysis. That process still needs much more thorough attention ... gruesome details of exactly how data are purified and selected for analysis, not to speak of details of how they are generated, which itself may involve, as equipment becomes increasingly complicated, many different independent tests of reliability and accuracy of equipment".

68. See Chapter 5 , Section 5.4 .2 for an example of the precision that such a theory of measurement operates with.

69. The issues concerned with this stage of science were addressed by Paul Galison in his book entitled How experiments end (1987), and the details have now been worked out to unbelievable depths in his follow-up /mage and logic (1997).

70. "The kind of co-ordinating definitions often described by philosophers have their place in popular philosophical expositions of theories, but in the actual practice of testing scientific theories a more elaborate and more sophisticated formal machinery for relating a theory to data is required" (Suppes in Morgenbesser, 1967; 62).

71. I agree with Wojcicki (in Humphreys, 1994:130) that Suppes's set-theoretical position may reduce philosophy of science to no more than "selected problems of metamathematics". Wójcicki writes: "Needless to say, as long as an empinical theory is not provided with any factual interpretation, it remains merely a certain formal system. But ... one may wonder whether the differentia specifica allowing us to tell an empirical theory from a piece of pure mathematics does not consist in the fact that the former has some intended empirical applications". He points out that this was essentially Adams's (1959) argument. He started the idea of an empinical theory consisting of two classes of structures, the one the class of all the theory's realisations and the other the class of all intended applications which is a class of empirical structures (physical systems) of which the theory is expected to be true. Adams also pointed out that not every intended application necessarily has to be a realisation of the theory. Sneed modified these notions in the sense that he requires that no component of an intended application be $T$ theoretical, while Adams saw the intended applications of structures of the same settheoretic type as the realisations of the theory itself.

72. I am, of course, not denying the use of statistical methodology to clarify and determine as precisely as possible the chances of a theory's models having connections to some systems in reality. It is merely the case that I rather advocate an elaboration of Van Fraassen's notion of empirical adequacy taking the place of the traditional literal notion of the "truth of a scientific theory" than the mathematical tools of statistics being employed to answer these inherently philosophical questions about possible relations between science and reality.

73. To be able to establish a representation theorem for a theory implies that it can be proved that there is a class of models of the theory such that every model of the theory is isomorphic to some member of this class. Suppes (1960:295) gives a few examples of such theorems, for instance, Cayley's theorem that every group is isomorphic to a group of transformations, and Stone's theorem that every Boolean algebra is isomorphic to a field of sets.

74. Suppes (1988b:254) claims that one of the most important and valuable uses of representation theorems in philosophy of science is that they help to increase (scientific) understanding of the represented object. Well, yes, if the conceptual models and their empirical subsets are of a different logical type, then obviously this may be the case. 
75. Or, in my terms, according to which the conceptual models and their empirical submodels are not necessarily of a different logical type, this simply means that, then, finally, the empirical interpretations of the theories in question may be examined.

76. If a representation theorem is found for one science in terms of a second, the first has been (formally) reduced to the second - e.g. Adams (1959) was the first to give a rigorous proof of reducing rigid body mechanics to particle mechanics.

77. The "type" of a model is determined by the individual constant symbols, as well as by the relation and function symbols of the axiomatic calculus of the theory in question.

78. The model-theoretic notions of both conceptual and empinical models also have this kind of clarifying effect. It is the case, as he (Suppes, 1989: 25) claims, that "A radically different situation often obtains in the companson of theory and expeniment. Theoretical notions are used in the theory which have no direct observable analogue in the experimental data. In addition, it is common for models of a theory to contain continuous functions or infinite sequences although the confirming data are highly discrete and finitistic in character. ... Corresponding to possible realisations of the theory, I introduce possible realisations of the data. As should be apparent, from a logical standpoint possible realisations of data are defined in just the same way as possible realisations of the theory being tested by the experiment from which the data come. The precise definition of models of the data for any given experiment requires that there be a theory of the data in the sense of the experimental procedure, as well as in the ordinary sense of the empirical theory of the phenomena being studied". He (Suppes, 1989:25) gives two reasons why a possible realisation of a theory cannot be a possible realisation of its data: no actual experiment can include an infinite number of discrete trials, and the parameter of the experiment is not directly observable and is not part of the recorded data. Note that although in a model-theoretic approach none of this is denied, this still does not necessarily imply that different languages are needed to talk about the content of the conceptual models and their empirical substructures.

79. Possible realisations of the theory cannot be possible realisations of the data (Suppes, 1989: 25), since no actual experiment can include an infinite number of discrete trials and the parameters of an experiment are not directly observable and are not part of the recorded data. In other words, models of the experiment and models of the theory are of a different logical type. This is not necessarily the case in a model-theoretic account of science, as I have already pointed out often in the above.

80. See Ruttkamp, 1997b, and the discussion related to these issues in Chapter 2.

81. Sneed (1983: 350 ) claims that structuralism "... is essentially a view about the logical form of the claims of empirical theories and the nature of the predicates that are used to make these claims". (The notion of 'predicates' is taken in the usual set-theoretic sense of characterising the type or species of sets of structures.)

82. This is perhaps where the real choice between the statement and the non-statement approach lies.

83. For formalised theories the entire (meta-) mathematical apparatus for studying theories and their models becomes available to the philosopher of science. One example of the tremendous usefulness of this approach is the study of verisimilitude. (See section 5.5.)

84. Both Stegmüller and Sneed formulated reconstructions of parts of Kuhn's theory, touching on the role of the scientific community in the development of scientific theories (Stegmüller, 1976; Sneed, 1976). Sneed claims that "... in order to make sense of what Prof Kuhn was telling us about scientific activity ... we found it convenient to ... employ a concept of scientific theory somewhat different from that commonly used by 
philosophers of science-in-general" (Sneed, 1976: 119). He is referring here to the adoption of the "non-statement view" by him and Stegmüller (and their followers). (Of course, Kuhn's work was not the only motivation in this regard, as Sneed himself acknowledges.) It is however interesting that such a precise formal reconstruction of Kuhn's incommensurability thesis as that offered by Sneed (1976) could have gone so relatively uncommented on in the philosophical community of the time. As already pointed out in the above. Sneed and Stegmüller both reconstructed parts of Kuhn's philosophy of science, focussing especially on the notion of a member of some community "holding" a particular theory, which implies a concentration on the differences between normal and revolutionary science.

Briefly, Sneed (1976: 120) defines "normal change" as change in the body of empinical claims of a theory, while "revolutionary change" consists in the changing of theories themselves. These notions can be very successfully treated by the structuralist programme, and Stegmüller (1973) specifically showed that the relation of reduction can be of significant use in depicting the notion of scientific progress.

85. Recall that in general, a model is a structure (interpretation) of the form $<A_{1}, \ldots, A_{m}, R_{1}$, $\ldots, R_{n}>$ where the $A$ are the "basic sets" or domains of the model (the ontology of the theory); and the $R_{\mathrm{j}}$ are relations on the $A_{i}$. Remember also that - at least for a language with a sound set of rules - satisfaction of the axioms implies satisfaction of the theory, for any interpretation.

86. We all know that a theory usually has many different models, but they all have one thing in common, which Balzer, Moulines and Sneed (1987: 3) identify as the same structure, while I emphasise also the fact that they are all models of the same (linguistically expressed) theory. A theory offers one formulation which binds together all these models (e.g. think of a theory as a set of field equations). That is why the model-theoretic approach is the one that I choose. This approach offers the possibility to focus on the linguistic nature of the theory as well as on its different models. Be that as it may, I do agree with Balzer, Moulines, and Sneed (1987: 3 ) that what is meant by models sharing the same structure, is that they all share the same conceptual framework (i.e. in my terms, they all have the same logical type or signature) and they all satisfy the same laws (theory).

87. Sometimes also referred to as "conceptual determinations".

88. "... this distinction may be understood as the model-theoretic explication of the distinction between the 'analytic' and the 'synthetic' components within a particular theory" (Moulines in Schurz \& Dom, 1991: 318). Or perhaps, in my terms, this may be viewed as the distinction between the themata and related context-specific factors, in so far as these co-determine the logical type (signature) of structures, and the linguistic formulation of the empinical claims suggested by the interpretation of the empirical data in question.

89. See Balzer, Moulines, Sneed (1987: 19, 20).

90. Briefly, the intensional description of $I$ is a description in terms of the properties of $I$, while the corresponding extension of the set $I$ denotes the elements of $I-$ i.e. which elements of $I$ have these (intensional) properties.

91. In my terms, the elements of $I$ would be representations of systems of the "real things".

92. Without a distinction between theoretical and non-theoretical terms, structuralists simply say that a particular intended application is an element of $M_{\rho}$. If such a distinction is made, they say that a particular intended application belongs to the class of partial potential models, $M_{P p}$, which is formally derivable from $M_{p}$. 
93. See Moulines in Schur \& Dom, 1991: 321, for an example of such an unwanted application. Also, recall Einstein's initial reaction to the models of his general theory of relativity implying a notion of the expansion of the universe rather than that of a static universe.

94. Or by any interpretation of models of theories in formal terms, for that matter.

95. Approximation has been left out of this discussion, simply because the inclusion of approximate relationships will only complexify matters needlessly, since this discussion is meant as a brief introduction into the structuralist programme. I think it suffices for my purposes to make it clear that within the structuralist programme all approximate relations can be defined formally and are definitely taken into account in their reconstructions of empirical theories.

96. See Moulines in Shur \& Dorn, 1991: 324.

97. This simply means that the set $I$ has "a life of its ${ }^{\circ} \mathrm{wn}^{\prime \prime}$ (ibid.), in the sense that its endurance is not dependent on the endurance of its members. The issue is especially complex, because the "life" or nature of the class I that endures through lime (or history) depends on the nature of the scientific community to which it is linked, which, in its turn, is also a "genidentical" entity.

98. The reason why the description of the set of intended applications has to be informal, is given by the representation theorem, referred to in the above mentioned quote from Balzer, Moulines, and Sneed (1987: 38). Note, however that although the set of intended applications cannot be depicted in purely formal terms, constraints and intertheoretical links can be formulated formally by using structural descriptions of models of the theory.

99. Sneed in Humphreys, 1994: 196 acknowledges this.

100. "Intended" here refers not to the formulation stages of theory development as I have set it out in Chapter 2, but rather Balzer et al. want to focus on the particular application (interpretation in my terms) of a specific theory to a certain real system or "range of phenomena".

101. Note again that Sneed and company take a theory olement as the core of a theory plus its range of intended applications. (See Sneed (1976).)

102. See Sneed (1976).

103. "The intuitive idea is that a distinction may be drawn between what is ruled out by the struclure of the theory's models $M$ and what is ruled out by restriction on the way that structure is applied 'across' a number of different applications $C^{\prime \prime}$ (Sneed, 1976: 124) - "Local applications [of theory T] may overlap in space and time, they may influence each other (even if they are separated in time and space), certain properties of T's objects may remain the same if the objects are transferred from one application to another one. Any connection of this sort will be captured by what we call constraints" (Balzer, Moulines, Sneed, 1987: 41).

104. See Balzer, Moulines, Sneed, 1987: 46ff., Sections II.2.3 and II.4 for more detail.

105. The sections of the structuralist programme dealing with these issues are very technical - see Balzer, Moulines, and Sneed, 1987: Section II.3.2 pp.57ff; and Section II.3.4 pp.73ff. for more detail.

106. See also Sneed (1976), and Baizer, Moulines, Sneed (1987). 
107. This is done (ibid.) in such a way that the whole array of theoretical components satisfies the constraints $C$.

108. See Balzer, Moulines, and Sneed (1987: 57ff).

109. All of the above is of course set out in idealised terms since it is not, in this context, taken into account that the empirical claim associated with a particular theory element will always - according to the structuralists - be only approximately true. What is relevant to this thesis in this connection is not the overwhelming literature on the technical aspects of the question of approximate truth, but rather, and much more simply, investigating exactly what the structuralists envisage the theory core's function to be in all of this. The briefest answer is, obviously, that the theory core identifies the theory content. More precisely, the theory core defines a set of possible situations or "ways things could be" (Sneed in Humphreys, 1994: 195), called content $(K)$. I shall not go into any more detail as far as these issues are concemed, given the scope of this thesis.

110. See Beth (1949), (1961), and Van Fraassen (1970).

111. See Suppe (1967), (1973), and (1989).

112. Note how such an approach focuses on the underdetermination of theories by models. This depiction of underdetermination differs though from that of a modeltheoretic account of science. See Chapter 5 for a discussion of these issues.

113. "Beth's approach does not require or presuppose the complete formalisation of the theory under analysis. ... His approach takes into account the essential role of models in science. In Beth's account, the mathematics is not part of the physical theory, but is used to construct the theoretical framework. The theoretical reasoning of the physicist is viewed as ordinary mathemalical reasoning conceming the framework. ... Finally, Beth's approach makes possible the use of formal semantic concepts and methods" (Van Fraassen, 1970:337, 338). Van Fraassen believes (1970:338) Beth's approach to be much closer to the actual foundational work done in physics than any variations of the statement approach.

114. Van Fraassen (1970) points out that Wilfred Sellars has since the late fifties been arguing for precisely such a meaning structure for the language of science. See Sellars (1957:225 - 308), and (1963, chapters 4, 10 and 11).

115. See Section 2.4 again.

116. The context-dependency of mathematical models does however also enter in their view of scientific theones in sofar as they - especially Suppe (1973: 151ff.) discuss the "extra-theoretical factors" determining for instance the experimental design of a theory. These factors include "regularities" (ibid.) such as other theories, laws or known regularities about the phenomena in question.

117. Giere (1983: 271) explains that a physical system "... is defined by a set of state variables and system laws that specify the physically possible states of the system and perhaps also its possible evolutions". Giere offers the example of classical thermodynamics which may be understood as defining an ideal gas in terms of three variables: pressure, volume, and temperature, and then specifying that these are related by the law $P V=K T$. (See also Suppe, 1973: 132.)

118. Van Fraassen (1970: 130-132) discusses three types of law:

- Laws of succession are relations of succession indicating the various sequences of states various physical systems will assume over time. These relations are such that 
the sequences may be deterministic or statistically determined, continuous or discrete. These laws (as far as they are non-statistical) thus select the physically possible trajectories in a particular state-space.

- Laws of coexistence are equivalence relations indicating which states are equivalent to which others, if the associated law is deterministic. If it is statistical it indicates which states are equally probable, i.e. it selects the physically possible subsets of the given state-space.

- Laws of interaction (either deterministic or statistical) determine which states result from the interaction between various systems. These laws are combinations of the first two kinds of law.

119. Suppe (1973: 136) elaborates on Beth's discussion of the usage of propositions and uses this elaboration to discuss the semantic relations between theory-formulation languages, theories, physical systems, and phenomena. He then defines a formulation of a theory as a set or class or "collection" of propositions which are true of the theory. Such a formulalion usually consists of a few specified propositions with all deductive consequences of the specified propositions under some "logic". These propositions are in a language called the "theory-formulation language", and usually forms a subset of the propositions of that language. The following basic features of a theory formulation may be identified (Suppe, 1973: 137 - 138):

- A set of elementary propositions in the theory-formulation language specifies that a certain physical parameter $p$ has as value a certain physical quantity $q$ at time $t$, such that an elementary proposition $\phi$ is true of state $s$ in the theory's domain if at time $t$, $s$ has $q$ as the value of the parameter $p$.

- For each elementary proposition $\phi$ there is a maximal subset $h(\phi)$ of the theory's domain such that $\phi$ is true of all the states in that subset.

- The function $h$ from elementary propositions to subsets of the theory is called the satisfaction function for the set of elementary propositions.

- Elementary propositions may be compounded together in accordance to the logic of the theory - the logic is such that every compound proposition is true of at least one state which is - according to the associated theory - physically possible; thus obviously the logic of a theory is theory-dependent and different theories may have different logics. (Suppe's (1973: 137) illustration: classical particle mechanics impose a Boolean algebra mod-2 and quantum theory imposes a non-distributive lattice.) In Beth and Van Fraassen's terms (Van Fraassen, 1970: 335) the logic of the theory is essentially a syntactic description of the set of valid sentences and the semantic entailment relation in that language.

- A language of description is determined by the set of elementary propositions, the theory, the satisfaction function $h$, and the logic of the theory; this language is obviously a sublanguage of the theory-formulation language; this language can describe any physicalfy possible state in a physical system.

- It might be possible that the logic of the theory enables one to deduce logical consequences of propositions in the language of description. However, usually the language of description has to be incorporated into a more complex language with an amended logic - namely none other than the theory-formulation language which can express the laws of the theory and deduce predictions.

- The truth conditions for the theory-formulation language are specified in terms of the relations (laws) of the theory and the truth conditions for the language of description.

- A formulation of a theory is a set of propositions deductively closed under the logic of the theory-formulation language such that every proposition in the set is true of the theory.

- Finally, the theory-formulation language may be a natural or an artificial language, but typically is a language such as "scientific English".

For analysing semantical relations holding between propositions in the expanded theory-formulation language and phenomena, Suppe (1973: 140ff.) offers a operationalist account of factual truth that I shall not discuss here. 
120. Especially non-relativistic physical theories typically use mathematical models to represent the behaviour of a certain kind of physical system - Van Fraassen $(1970: 328)$ gives as examples the use of Hilbert space in quantum mechanics, and the use of Euclidean $2 n$-space [sic.] as phase-space for $n$ particles in classical mechanics. (He probably means ".... for a system with $n$ degrees of freedom ....". For $n$ particles $6 n$-space is neededl)

121. This is very much in agreement with my idea of a (conceptual) model of a theory and relations linking it to some real system via some empincal model offering a "snap shot" view of the real system at a specific time.

122. "For each elementary statement $U$ there is a region $h(U)$ of the state-spaces $H$ such that $U$ is true if and only if the system's actual state is represented by an element of $h(U)$. (We also say that these elements satisfy $U . .$. )" (Van Fraassen, 1970:329).

123. This notion of a "satisfaction function" characterises exactly the kind of relation I see involved in determining the possible isomorphic embeddings of empirical models into conceptual models of some theory.

124. Van Fraassen's semi-interpreted language thus in these terms consists of the elementary statements connected to a certain physical system, the specific statespaces in question, and the satisfaction function in question.

125. See Van Fraassen (1970: 337).

126. "For our purposes, a scientific theory has two components. One is a family of [theoretical] models ... . The second is a set of theoretical hypotheses that pick out things in the real world that may fit one or another of the models in the family" (Giere, 1991:29).

127. See my discussion of this issue in Chapters 4 and 5.

128. Think of Suppes's hierarchy of models. See also my discussion of the underdetermination issue in Chapter 5.

129. See Wartofski (1979: 19).

130. He (Giere, 1984:13) gives as example of a theoretical mypothesis the statement that "The positions and velocities of the earth and moon in the earth-moon system are very close to those of a two-body particle Newtonian model (with specified initial conditions)".

131. Another example of a notion of approximation by degree, as noted by Giere $(1985: 80)$, can be found in Beth's state-space approach in terms of the value $r$ of a magnitude $m$ at a time $t$ in a given state-space. This notion also fits both my and Suppes's approaches to these "empirical" relations.

132. "I agree with contemporary students of probability, induction, and the foundations of statistics that the individual hypothesis is a useful unit of analysis. On the other hand, I reject completely the idea that one can reduce the rationality of the scientific process to the rationality of individual agents. The rationality of science is to be found not so much in the heads of scientists as in the objective features of its methods and institutions" (Giere, 1983:270). He also (like Suppes, Van Fraassen, and Suppe) reflects on the complex nature and role of data in the testing of theoretical hypotheses: "... in order to determine whether a proposed model fits the world one needs some information about the part of the world in question. But not all information is relevant. We will use the term data ... to refer to the special information 
that may be relevant to deciding whether the model in question does fit" (Giere, 1991:29). Giere (1991:37-40) offers a detailed programme for the evaluation of theoretical hypotheses, but I will not go into the finer points of that here.

133. Wójcicki's empiricism has some far reaching consequences that could not have been accommodated by classic empinicism. Newer forms, like that of Van Fraassen, are more flexible though. The classic version views observations in terms of the fixed point of view of the idealised "normal objective observer", while Wojcicki now offers a more flexible empiricism in so far as the experimental accessibility of specific states of affairs depends heavily on the competence of the experimenter and her technical capabilities.

134. See my comments on this matter above, and in Ruttkamp (1997a, 1997b).

135. This is Suppes's notion of a "physical" model and my notion of an "intended" model.

136. See Wójcicki's examples in this regard in Humphreys, 1994:134 - 136.

137. See Wójcicki in Humphreys, 1994:135.

138. "The fact that formation of a theoretical model presupposes formation of a model of the data as well as the fact that formation of a model of the data can be controlled by the requirement of consistency of the model with the corresponding theory are of key significance for proper understanding of the interplay between the data and the theories, and thus for proper accounting for both the corrigibility of the data and the falsifiability of the theoretical claims" (Wójcicki in Humphreys, 1994:137).

139. Wójcicki (in Humphreys, 1994: 138) writes "... let me point out that theoretical models are not empirical systems, unless we consider them to be empirical systems of some hypothetical or possible worlds. The reason is quite obvious. Even though the objects a model involves are supposed to be real ... still these objects are postulated to satisfy certain theoretical assumptions ... which they may not actually satisfy. A theoretical model is just a certain theoretical construct, an abstract entity, even if some of its parts appear to be parts of physical reality". See also Endnote \#9 and Chapter 3 where 1 - referring to Suppes and Moulines - already drew atterition to questionable attempts to mix conceptual and physical objects (by making the latter elements of mathematical sets).

140. Wójcicki (in Humphreys, 1994:140) apparently does see this, though: "There is no question that, on many occasions, the search for a theoretical model for a question $Q$ is the search for a semantical model of a specific theory $T$ such that this particular model will be the right one to examine question $Q$. This is the situation that obtains whenever one believes that $T$ is the theory which allows for solving $Q$. If the formation of a theoretical model is from the very beginning meant to consist in selecting the right semantical model then, of course, the notion of a semantical model is prior to that of a theoretical one. ... Newtonian particle mechanics was viewed as the right theory to solve the question $Q_{t}$ conceming Mercury's movement. However there are numerous occasions when the search for a model for $Q$ starts when one does not consider any theory to be directly applicable to $Q$. Bohr's model of the atom, which in fact was a model of some specific problems on the dynamics of the particles of which the atom was believed to be composed, was certainly not meant to be a semantic model of the official theory; rather it was meant to be a certain tentative theory of the phenomenon. ... [Here] [t]he construction of a theoretical model precedes any semantic considerations and in this sense it is prior to the latter. ... the idea of a model is central for two, in a serise, opposite activities: applying theories and forming them. The interplay between the two is what largely determines the dynamics of science... Now, while the concept of semantic model is 
central for the former, the theoretical model is central for the latter".

141. This is what Wojjcicki (1979: 159) is getting at when he remarks that "... there is an evident need for a term which both when a theory is being constructed and when it is finally set up can be applied to denote those parts of the theory that provide a relatively complete account of particular regularities of phenomena in the scope of the theory".

142. See also Wójcicki's (1979: 161 - 163) example illustrating these problems.

143. This implies that propositions in the theory-formulation language may refer to and describe some physical system because a physical system in the theory-induced class of systems restricts the theory to a single sequence of states.

144. These notions form the core of Giere's "constructive realism" (Giere, 1985).

145. Nancy Cartwright's recent attacks (1989, 1994a, 1994b, 1995a) on "fundamentalism" seem rather pointless from this viewpoint (see the next chapter).

146. Some sections of this chapter will be published in Ruttkamp (1999a) and (1999b).

147. And so they should be, given that they form part of human scientific knowledge which, from the beginning, simply is based on activities of abstraction, because that simply is how we humans know anything.

148. My italics.

149. We have already agreed that fundamental laws are indeed too simple and abstract to be directly about any aspect of reality.

150. She also remarks in Cushing, Delaney, and Gutting (1984: 135) that "... abstractness and scientific realism are two different issues, and not all varieties of abstractness bear equally on questions of descriptive completeness, accuracy, and truth. This is so with our notion [of abstraction], where notions become more and more abstract as less and less explanatory information about them is given".

151. Both Cartwright and I view these models as idealisations, although we differ about the implications of the ideal nature of these models for the process of science, as will be discussed below.

152. Cartwright, 1983 , chapter $B$.

153. Cartwright's "phenomenological laws" remind one very much of Suppes's (1989) models of data.

154. I shall elaborate on this in Section 4.3 .

155. See also Chalmers $(1987 ; 82)$ for confirmation of this interpretation.

156. See Chapter 5.

157. Well, this is true of fundamental laws too - Newton says openly he offers no hypotheses conceming the reasons why his laws of gravitation are true.

158. See also my notes concerning Newton's mechanics offering an explanation of Kepler's laws in Chapter 2. 
159. I cannot go along with Laymon's "normative description" of explanation though, since the "truth" of fundamental laws can only be "discovered" by studying the models interpreting them and investigating their empirical adequacy.

160. Remember that Cartwright takes scientific theories to be a set of fundamental laws - like Maxwell's equations - from which explanations in physics are supposed to start.

161. This is one more reason for the importance of theories or fundamental laws in the process of science. One theory may have infinitely many "incompatible" models (i.e. models that are not isomorphic) and "the facts" may be valid in all of these. Even though Cartwright will probably not agree that it is possible to give a realist slant to the double underdetermination of models, it shows clearly a way in which the notion of theories seems indeed to have an organising and classifying nature in her terms.

162. Cartwright claims (1983: 150) a model realistic in this second sense to be in need of more bridge principles than one realistic in the first sense. The best explanation for this is, I think, the fact that she sees the mathematical representation as being closer to - or perhaps mainly identical to - the theory.

163. As she explains (Cartwright, 1983: 152-154): "The second definition of 'simulacrum' in the Oxford English Dictionary says that a simulacrum is 'something having merely the form or appearance of a certair thing, without possessing its substance or proper qualities"'.

164. She offers Maxwell's treatment of the radiometer as a further example - Cartwright, 1983: 154-155.

165. Cartwright illustrates this accusation with the following remarks: "Not all radiometers that meet Maxwell's two descriptions have the distribution function Maxwell writes down; most have many other relevarit features besides. This will probably continue to be true no matter how many further corrections we add. In general ... the bridge law between the medium of a radiometer and a proposed distribution can hold only ceteris paribus" (Cartwright, 1983: 155).

166. There are cases in which we believe phenomenological laws to be soundly deducible from a certain set of fundamental laws, but find that the actual deduction is extremely difficult. These cases, however, do not prove in any way either that phenomenological laws "typically" cannot be deduced from fundamental ones, or that the "all things being equal" and additional assumptions needed in such deductions may be found to "contradict" the original (set of) fundamental law(s).

167. Cartwright offers the problem of superconductivity as illustration - see Cartwright, Shomar and Suárez, 1995: 142 - 149.

168. Note that, in principle, it is possible to deduce the movement of "Neurath's bill" perfectly from Newton's laws. The real system is in this case simply too complex to be able to actually make this deduction in practise.

169. Cartwright refers to this too.

170. As the criticism of "fundamentalist realist" theories of science aptly shows.

171. She offers the finer detail of her arguments supporting this claim in Nature's capacities and their measurement (1989) by distinguishing between abstraction, idealisation, and concretisation, and offening a discussion of the problem of "material abstraction" (which refers to the problems arising from the fact that no universal 
recipe can be given for the movements from theory to concrete situations).

172. In a nutshell, that is what this thesis is all about.

173. While working in her usual context of theoretical physics, Cartwright most often views theories very mathematically, as sets of field equations or whatever.

174. This notion of capacities is close to Popper's propensities. See Popper (1990).

175. In addressing the testability of causal claims, Cartwright uses probabilities, while the Humean tradition reduced causal laws to probabilities, She says: "I defend a very different understanding of the concept of Natural Law in modem science from the 'Laws = universal regulanities' account ... We aim in science, I urge, to discover the natures of things; we try to find out what powers or capacities they have and in what circumstances and in what ways these capacities can be harnessed to produce predictable behaviours. I call this the study of natures because I want to recall the Aristotelian idea that science aims to understand what things are, and a large part of understanding what they are is to understand what they can do, regularly and as a matter of course. Regularities are secondary. Fixed patterns of association among measurable quantities are a consequence of the repeated operation of factors that have stable capacities (factors of this kind are sometimes called 'mechanisms') arranged in the 'right' way in the 'right kind' of stable environment" (Cartwright, 1995c: 277). Ceteris paribus clauses can, however, it seems, not be escaped - "In order to generate a prediction [or, give an explanation] we must figure out how to combine the laws together and how to cash-out their ceteris paribus conditions and we musi do 50 in a way that takes into account the specific material circumstances of the situation under consideration" (Cartwright, 1995a: 155). The way to do this then, is to assume the existence of capacities (as has already been pointed out) - "The point is that the fundamental facts about nature that ensure that regularities can obtain are not again themselves regularities. They are facts about what things can do" (Cartwright, 1995a: 156).

176. For instance, she claims that standard philosophical accounts of probabilistic causality actually employ a concept of causation that is much stronger than the concept of a mere causal law and gives (Cartwright, 1989: 142) the following

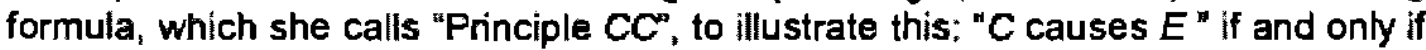
the probability of $E$ is greater with $C$ than without $C$ in every causally homageneous context. According to her (1989: 143) the point with regard to this formula is that it is universally quantified, and thus represents a concept of causality powerful enough to be taken as a concept of capacity as well.

177. See Cartwright (1989: 193).

178. Some sections of this chapter will be published as Ruttkamp (1999b).

179. In a recent spirited defense of "causal realism" Chnistopher Norris (1997) also offers a criticism of the anti-realism implied by Van Fraassen's "constructive realism". (See Norris, 1997, chapters $6 \& 7$.)

180. I have already briefly commented on this issue in Chapter 4.

181. See Spurrett (1998) for a thorough discussion of this aspect of Bhaskar's transcendental realism.

182. Hacking (1983: 3) also sets out the debate in these terms. 
183. Speaking of the "contingent" nature of data is not meant to sound frivolous, but rather to refer to the verification of the validity of data via the various models of some scientific theory.

184. Here I agree with Kuhn's (1977: 267) description of the "intimale and inevitable entanglement of scientific observation with scientific theory" that leads - as Kuhn (ibid.) also remarks - to a certain skepticism conceming the production of a "neutral observation language".

185. I thank Professor Johannes Heidema of the Department of Mathematics, Applied Mathemalics, and Astronomy at the University of South Africa for this metaphor.

186. Nancy Nersessian (1984: 153) wriles that a discussion of a concept of meaning that would be adequate for scientific theories should be given in terms of the following two factors. The study of nature and an analysis of language, as well as an analysis of "actual scientific practices concerning meaning" (ibid.). She (ibid.) claims that it is impossible to separate questions of meaning from the "network of beliefs (theoretical, methodological, melaphysical, common sense) and problems (theoretical, experimental, and metaphysical) which provides the 'motive force' in meaning construction" (ibid.). She (Nersessian, 1984: 156) continues to describe the meaning of a scientific concept as "a two-dimensional array which is constructed on the basis of its descriptive/explanatory function as it develops over time. I will call this array a 'meaning scheme"'. (This reminds somewhat of Davidson's (1984) "conceptual schemes".) The need for something like any of these notions of meaning or conceplual schemes in examining the process and progress of science is taken care of in a model-theoretic account of science in terms of intended, conceptual, and empinical models of scientific theories.

\section{See Chapter 2.}

188. I shall not say anymore on this last point - i.e. the additional metaphysical content of realist theories. I have already pointed out during the discussion of Cartwright's notion of capacities and Bhaskar's notion of tendencies above, that modeltheoretically speaking these kinds of musing are not necessary at all. The discussions of the relations between science and reality in the first parts of this chapter also support this claim. The first two sets of problems - i.e. approximate truth and related issues, and underdetermination - will be discussed in Section 5.5.

189. Suppe (1973:161) defines a theory formulation as a set of propositions which are true of the theory. Such a formulation usually seems to consist of a few specified propositions with all the deductive consequences of those propositions under some "logic" of the theory.

190. A science like physics cannot exist at all if it were not to use rich mathematical structures and the deductive methodology of mathematics and restrict itself to empirical models only. For example, no physical measuring process can ever produce an irrational number as result, although interpreting some of these results necessanily assumes the existence of such numbers.

191. Bhaskar uses the terms "transduction" or the "transfactual nature of laws" to refer to the applicability of scientific laws specifically outside the domain of actual experience. un the full analysis of law-like statements we are thus concerned with a new kind of conditional ... [These conditionals] take us to a level at which things are really going on irrespective of [their] actual outcome" (Bhaskar 1978: 51). These "normic" conditionals are transfactual rather than counterfactual because they don't describe what would happen but rather what is happening, albeit in an unmanifested way. Bhaskar (1986:100) claims a scientist is always certain that, given some effect, 
something is producing the effect in question - her only doubt is about what exactly that "something" may be. In this sense, as claimed above, the function of scientists is primarily to produce a theory which correctly describes - or adequately explains - the mechanism by means of which the effect in question is produced. In asserting a "nomic" statement, one is asserting the operation of a mechanism, imespective of its results (here is referred to the fact that tendencies of mechanisms do not have to become manifest or be realised or actualised in open - i.e. "real" - systems), Hence, the fact that our knowledge can be both universally applicable and rarely instantiated can only become intelligible if we presuppose the (transitive and intransitive) stratification of the world.

192. See Chapter 3.

193. See Clarke (1998) as well.

194. Philosophers such as Richard Boyd (1994) and Eman McMullin (1993) also endorse the notion of approximate truth, although they each offer their own version of it.

195. The inherent problems of the notion of approximate truth are clearly discussed in Laudan's (1981b) A confutation of convergent realism. See also Part I of Donovan, A., L. Laudan, \& R, Laudan (1988).

196. Laudan (in Cushing, Delaney, and Gutting, 1984: 91) offers the example of Newtonian optics in this respect. There is nothing in reality that corresponds - not even approximately - to Newtonian corpuscles. Think also of the well-known notion of ether as the medium through which light moves that was in place for hundreds of years in various forms, and was finally thrown out of the framework of science by Einstein's notions of special and general relativity. One need not concur with Laudan on this point. There is much more continuity over time in the models relevant to these examples than he seems to give credit for. Put simply: the present-day view of photons of light has much in common with Newton's model of light corpuscles. Similarly today's fields in space-time share many of the functions of the ether. Modeltheoretic realism ascribes these common features of models (of successive theories) over time to their empirical links to features of systems in reality. (See also in Chapter 2 my discussion of the nature of scientific progress in model-theoretic terms.)

197. Note for instance that although the Ptolemaic theory's predictions were more accurate than the Copernican heliocentric theory of our solar system, Copernicus's theory still "eliminated" the Ptolemaic one. How can such occurrences in science's processes be explained? Well, an established theory (or model of some theory) has a whole foundational structure of developments and refinements to lean on that a "new" theory (or model) does not immediately have at its disposal. Although it is generally taken that a "newer" theory can explain "more" than an "older" theory, it is not necessarily the case that the level of accuracy of the "new" theory will be as high as that of the "old" theory from the beginning. Also, it must be kept in mind that the accuracy of the "old" theory (or model) might be an "unnatural" accuracy, in the sense of resuscitating - in Lakatos's terms - already degenerative research programmes via ad hoc additions.

198. See Lakatos, (1978).

199. Intertheoretic relations are for instance equivalence, theorelisation, and reduction.

200. Sneed (1983: 364 ) describes the notion of a theoretical individual kind term as follows: "Intuilively, a TIKT for a given pre-theoretically described set of intended applications $I$ 'denotes the set of all theoretical individuals that could be used in some 
way to make members of $I^{\prime}$ into $M_{p p} s^{\prime \prime}$.

201. This is essentially my notion of empirical adequacy too - i.e. that theories with the same empirical models (although they may be embedded into different conceptual models) are empirically equivalent.

202. Van Fraassen (1980:60) also remarks on the fact that in the various axiomatisations of mechanics offered during our century - such as the one offered by McKinsey, Sugar \& Suppes (1953) - the terms "mass" is treated in almost as many ways. Another effective - and perhaps more interesting - example of these issues is given by the measurement problems in quantum mechanics which is even more complex as far as the underdetermination of the underlying microscopic state by the observable macroscopic phenomena is concerned. Van Fraassen $(1980: 62,63)$ discusses this issue as well.

203. See Chapter 3.

204. Note that, in principle, the language in question need not be a formal language at all. Almost any kind of scientific linguistic expression may be formalised in some firstorder language and its interpretations re-constructed in a model-theoretic way.

205. Briefly, Putnam's argument comes down to the following: Take $T_{\text {, }}$ to be an "ideal" theory in all possible senses, except that it is objectively true. Assume that "THE WORLD" can be divided into infinitely many pieces. Take $T$, to claim that there are infinitely many things (such that, in this case, $T_{1}$ is objectively correct about the world). Then, by the completeness theorem, and given that $T_{1}$ is consistent and has only infinite models, $T_{1}$ has a model of every infinite cardinality. Then, if we choose a model $M$ of the same cardinality as "THE WORLD" and we map the individuals of $M$ one-to-one into the pieces of "THE WORLD", and we then use this mapping to define relations of $M$ directly onto "THE WORLD", the result is a satisfaction relation "SAT" which is a "correspondence" between the terms of the language $L$ and the sets of parts of "THE WORLD" such that $\mathrm{T}_{1}$ comes out true of "THE WORLD" - provided that "true" is interpreted as meaning TRUE(SAT). The paradoxical implication of Putnam's argument is that if the criteria we formulate to determine the truth of certain sentences are indeed solely intemal (implying that these sentences "must come out true" (Van Fraassen, 1997: 18)), then "practically any theory is true" (ibid.). l.e. the dilemma is - or seems to be - that if we cannot talk about "THE WORLD" then we cannot say anything about the extensions to our predicates in "THE WORLD"; but if we can indeed describe - talk about - elements in "THE WORLD", then we can distinguish between right and wrong assignments of predicates of extensions to our predicates in a rather trivial manner. See Putnam (1978), Lewis (1984), and Van Fraassen (1997) for more detailed definition and discussion of this argument.

206. E.g. Galison (1987) and (1997) offer a treasure trove for the philosopher in this respect.

\section{See Chapter 5.}

208. Think of the notion of so-called "picture theories", and of logical atomism, for instance.

209. This seems to be the basic idea of Kripke's Naming and necessity (1980).

210. Of course she also is referring to the idealised nature of the models interpreting the fundamental laws of physics when she speaks of the falsity of these laws. That is however a different issue, addressed already in Chapter 4. This does not take away though the issue concerning her attitude towards the reference of the theoretical 
terms in these laws.

211. See Lewis (1970) for a good illustration of the syntactic scheme of things.

212. Perhaps the reason for this is simply the historical psychological "feeling" that numbers and sets are somehow unique entities (floating around somewhere).

213. Abraham Robinson (for instance 1986) developed the model-theoretic structure of algebra in this way.

214. See Chapters 2 and 5.

215. Hintikka (1989: 55) remarks that this domain can be so particular that it can be characterised as "... a 'small world", that is, a relatively short course of local events in some nook or comer of the actual world". 


\section{BIBLIOGRAPHICAL REFERENCES}

Achinstein, P. 1968. Concepts of science: A philosophical analysis. Baltimore: John Hopkins Press.

Adams, E.W. 1959. The foundations of rigid body mechanics and the derivation of its laws from those of particle mechanics. In Henkin, L., P. Suppes \& A. Tarski. (Eds). 1959. The axiomatic method. Amsterdam: North-Holland: $250-265$

Balzer, W., C.U. Moulines, \& J.D. Sneed. 1987. An architectonic for science - The structuralist programme. Dordrecht: D. Reidel.

Bachelard, G. 1934. The new scientific spirit. Boston: Beacon Press.

Beth, E.W. 1948/49. Analyse sémantique des théories physiques. Synthese, Vol. 7: 206 207.

Beth, E.W. 1949. Towards an up-to-date philosophy of the natural sciences. Methodos, Vol. 1: $178-185$.

Beth, E. W. 1961. Semantics of physical theories. In Freudenthal, H. (Ed). 1961. The concept and the role of the model in mathematics and natural and social sciences. Dordrecht: D. Reidel \& Co: 48 - 51.

Bhaskar, R. 1978. (2 ${ }^{\text {nd }}$ ed, ) A realist theory of science. Sussex: Harvester.

Bhaskar, R. 1979. The possibility of naturalism: a philosophical critique of the contemporary human sciences. Sussex. Harvester.

Bhaskar, R. 1986. Scientific realism and human emancipation. London: Verso.

Bhaskar, R. 1989. Reclaiming reality. a critical introduction to contemporary philosophy. London: Verso.

Boyd, R. 1994. What realism implies and what it does not. In Worrall, J. (Ed), 1994. The ontology of science. Aldershot: Dartmouth; 71 - 96.

Brink, C. \& J. Heidema. 1987. A verisimilar ordering of theories phrased in a propositional language. The British joumal for the philosophy of science, Vol. 38: 533 - 549.

Burger, I.C. \& J. Heidema. 1994. Comparing theories by their positive and negative contents. The British joumal for the philosophy of science, Vol. 45: 605 - 630.

Carnap, R. 1958. Beobachtungssprache und theoretische Sprache. Dialectica, Vol. 12: 236 248.

Cartwright, N. 1979. Causal laws and effective strategies. NOŨS, Vol. 13: 419 - 437,

Cartwright, N. 1983. How the laws of physics lie. Oxford: Oxford University Press.

Cartwright, N. 1986. Fables and models. In: Worrall, J. (Ed). 1986. The ontology of science. Aldershot: Dartmouth: 191 - 204.

Cartwright, N. 1989. Nature's capacities and their measurement. Oxford: Clarendon Press. Cartwright, N. 1991a. Can wholism reconcile the inaccuracy of theory with the accuracy of 
prediction? Synthese, Vol. 89: 3 - 13.

Cartwright, N. 1991b. The reality of causes in a world of instrumental laws. In Boyd, R., P. Casper \& J.D. Trout. 1991. The philosophy of science. Massachusetts: MIT Press: 379 - 386.

Cartwright, N. 1994a. In defence of this worldly causality. Comments on Van Fraassen's Laws and symmetry. Philosophy and Phenomenological Research, Vol. 53(2): 423 - 429.

Cartwright, N. 1994b. Fundamentalism and the patchwork of laws. Proceedings of the Aristotelian Society, Vol. 94: 279 - 292.

Cartwright, N. 1994c. Is natural science natural enough? A reply to Philip Allport. Synthese, Vol. 94(2): 291 - 301.

Cartwright, N. 1995a. Précis of "Nature's capacities and their measurement. Philosophy and Phenomenological Research, Vol.55(1): 153 - 156.

Cartwright, N. 1995b. False idealisation: A philosophical threat to scientific method. Philosophical Studies, Vol. 77(2-3): 339 - 352.

Cartwright, N. 1995c. Ceteris Paribus laws and socio-economic machines. The Monist, Vol. 78(3): 276 - 294.

Cartwright, N. 1997. Why physics? In Penrose, R. (Ed). 1997. The large, the small, and the human mind. Cambridge: Cambridge University Press: 161 - 168.

Cartwright, N. \& J. Dupré. 1988. Probability and causality: Why Hume and indeterminism don't mix. NOÜS, Vol. 22: 521 - 536.

Cartwright, N. \& H. Mendell. 1984. What makes physics' objects abstract? In Cushing, J.T., C.F. Delaney, \& G.M. Gutting. (Eds). 1984. Science and reality. Recent work in philosophy of science. Indiana: University of Notre Dame Press: 134 - 152.

Cartwright, N., T. Shomar \& M. Suárez. 1995. The tool-box of science. In: Herfel, W.E., W. Krajewski, I. Niiniluoto \& R. Wöjcicki. (Eds). Theories and models in scientific processes. Poznán studies in the philosophy of sciences and the humanities. Vol. 44: 137 - 149.

Chalmers, A. 1987. Bhaskar, Cartwright and realism in physics. Methodology and science, Vol. 20: 77 - 96 .

Chalmers, A. 1993. So the laws of physics needn't lie. The Australasian journal of Philosophy, Vol. 71(2): 196 - 205.

Clarke, S. 1998. Fundamentalism, laws, causes and lies. In Clarke, S. 1998. Metaphysics and the disunity of scientific knowledge. Aldershot: Ashgate: 18 - 54.

Cook, A. 1994. The observational foundations of physics. Cambridge: Cambridge University Press.

Da Costa, N.C.A. \& S. French. 1990. The model-theoretic approach in the philosophy of science. Philosophy of science, Vol. 57: 248 - 265.

Davidson, D. 1984. The very idea of a conceptual scheme. In Davidson, D. 1984. Inquinies into truth and interpretation. New York: Oxford University Press: 183 - 198.

Dilworth, C. 1994a. The set-theoretic conception of science. Dilworth, C. 1994. ( $3^{\text {rd }}$ ed.) 
Scientific progress. A study conceming the nature of the relation between successive theories. Dordrecht: Kluwer Academic Publishers: 118 - 132.

Dilworth, C. $1994 \mathrm{~b}$. Application of the perspectivist conception to the views of Newton, Kepler, and Galileo. In Dilworth, C. 1994. ( $3^{\text {rd }}$ ed.) Scientific progress. A study concerning the nature of the relation between successive theories. Dordrecht: Kluwer Academic Publishers: 133 141.

Dilworth, C. 1994c. On theoretical terms. Dilworth, C. 1994. (3 $3^{\text {rt }}$ ed.) Scientific progress. A study conceming the nature of the relation between successive theories. Dordrecht: Kluwer Academic Publishers: 142 - 156.

Donovan, A., L. Laudan, \& R. Laudan. 1988. Testing theories of scientific change. In Donovan, A., L. Laudan, \& R. Laudan. (Eds). 1988. Scrutinising science. Empirical studies of scientific change. Dordrecht: Kluwer: 3 - 46.

Duhem, P. 1914. The aim and structure of physical theory. Princeton: Princeton University Press.

Einstein, A. 1956. $\left(5^{\text {th }}\right.$ ed.) The meaning of relativity. New Jersey: Princeton University Press,

Fine, A. 1986a. Unnatural attitudes: Realist and instrumentalist attachments to science. Mind, Vol. XCV(378): $149-179$.

Fine, A 1986b. The shaky game: Einstein, realism, and the quantum theory. Chicago: Chicago University Press.

Galison, P. 1987. How experiments end. Chicago: University of Chicago Press.

Galison, P. 1997. Image and logic: A material cutture of microphysics. Chicago: University of Chicago Press.

Gamov, G. 1962. Biography of physics. London: Hutchinson.

Giere, R.N. 1983. Testing theoretical hypotheses. In Earman, J. (Ed). 1983. Testing scientific theories. Minnesota studies in the philosophy of science, Vol. X. Minneapolis: University of Minnesota Press: 269 - 298.

Giere, R.N. 1984. Toward a unified theory of science. In Cushing, J.T., C.F. Delaney \& G. M. Gutting. (Eds). 1984. Science and reality: Recent work in the philosophy of science Indiana: University of Notre Dame Press: 5 - 31.

Giere, R.N. 1985. Constructive realism. In Churchland, P.M. \& C. A. Hooker. (Eds). 1985. Images of science: Essays on realism and empiricism. Chicago: University of Chicago Press: $75-98$.

Giere, R.N. 1991. Understanding scientific reasoning. New York: Harcourt Braca Jovanovich College Publishers,

Giere, R. N. 1994. The cognitive structure of scientific theories. Philosophy of science, Vol. 2: $276-296$.

Grünbaum, A. 1954. Science and ideology. The scientific monthly. July Edition: 13 - 19.

Haalvelmo, T. 1944. The probability approach in econometrics. Econometrica, Vol. 12 (supplement). 
Hacking, I. 1983. Representing and intervening. Introductory topics in the philosophy of natural science. Cambridge: Cambridge University Press.

Hacking, I. 1997. Experimentation and scientific realism. In Tauber, A.l. (Ed). 1997. Science and the quest for reality. London: Macmillan Press Ltd: 162 - 181.

Harrod, J. 1968. What is a model? In Wolfe, J. N. (Ed). 1968. Value, capital and growth. Papers in honour of Sir John Hicks. Edinburgh: University Press: 173 - 191.

Hausman, D.M. 1992. Models and theories in economics. In Hausman, D. M. 1992. The inexact and separate science of economics. Cambridge: Cambridge University Press: 70 101.

Heidema, J. \& H.J. Schutte. 1978. Truth criteria in deductive theories. Philosophical papers, Vol. 7: $51-68$

Hesse, M. 1963. Models and analogies in science. Oxford: Oxford University Press.

Hintikka, J. 1989. Exploring possible worlds. In Allèn, S. (Ed). 1989. Possible worlds in humanities, arts and sciences. Proceedings of Nobel Symposium 65. Berlin: Walter de Gruyter.

Holton, G. 1995. The role of themata in science. Foundations of physics, Vol. 26(4): 453 465.

Kieseppă, I. 1996. Truthlikeness for multidimensional, quantitative cognitive problems. Dordrecht: Kluwer.

Kripke, S.A. 1980. Naming and necessity. Oxford: Blackwell.

Kuhn, T.S. 1970. (2 $2^{\text {nd }}$ ed.) The structure of scientific revolutions. Oxford: Oxford University Press.

Kuhn, T.S. 1977. The essential tension. Selected studies in scientific tradition and change. Chicago: University of Chicago Press.

Kuhn, T.S. 1997. The road since structure. In Tauber, A.I. (Ed). 1997. Science and the quest for reality. London: Macmillan Press Ltd: 231 - 248.

Kuipers, T.A.F. 1987. What is closer-to-the-truth? Amsterdam: Rodopi.

Kuipers, T.A.F. 1992. Naive and refined truth approximation. Synthese, Vol. 93; $299-341$.

Kuipers, T.A.F. 1994. The refined structure of theories. In Kuokkanen, M. (Ed). 1994. Poznan studies in the philosophy of the sciences and the humanities, Vol. 42: Idealisation IV: Structuralism, idealisation, and approximation. Amsterdam: Rodopi: $3-24$.

Kuipers, T.A.F. 1997. The dual foundation of qualitative truth approximation. Erkenntnis, Vol. 47: 145 - 179 .

Lakatos, I. 1978. The methodology of scientific research programmes. Cambridge; Cambridge University Press.

Laudan, L. 1981a. A problem-solving approach to scientific progress, In Hacking, I. 1981. Scientific revolutions. New York: Oxford University Press: 144 - 155. 
Laudan, L. 1981b. A confutation of convergent realism. Philosophy of science, Vol. 48: 19 49.

Laudan, L. 1984. Explaining the success of science: Beyond epistemic realism and relativism. In Cushing, J.T., C.F. Delaney, \& G.M. Gutting. (Eds). 1984. Science and reality. Recent work in philosophy of science. Indiana: University of Notre Dame Press: 83 - 105.

Laymon, R. 1989. Cartwright and the lying laws of physics. The jouma/ of philosophy, Vol.86: $353-372$

Lenk, H. 1993. Interpretation und Realität. In Lenk, H. 1993. Philosophie und Interpretation. Frankfurt am Main: Suhrkamp: 264 - 272.

Lenk, H. 1995. Bezugs(h)erstellung: Interpretationistischgebrauchstheoretische Theorie der Referenz. In Lenk, H. 1995. Schemaspiele. Ober Schemainterpretationen und Interpretationskonstrukte. Frankfurt am Main: Suhrkamp: 132 - 156.

Leplin, J. 1997. A novel defence of scientific realism. Oxford: Oxford University Press.

Lewis, D. 1970. How to define theoretical terms. The joumal of philosophy, Vol. $67: 427-446$.

Lewis, D. 1984. Putnam's paradox. The Australasian joumal of philosophy, Vol. 62: 221 - 236.

Ludwig, G. 1990. (2 $2^{\text {nd }}$ ed.) Die Grundstrukturen einer physikalischen Theorie. Berlin: Springer Verlag.

Margolis, J. 1995. Historied thought, constructed world. A conceptual primer for the tum of the millennium. Berkeley: University of California Press.

McKinsey, J.C.C. \& P. Suppes. 1953. Philosophy and the axiomatic foundations of physics. In Proceedings of the Xlth International Congress of Philosophy, Vol. 6: $49-54$.

McKinsey, J.C.C., A.C. Sugar, \& P. Suppes. 1953. Axiomatic foundations of classic particle mechanics. The joumal of rational mechanics and analysis, Vol. 2: $253-272$.

McMullin, E. 1993. Rationality and paradigm change in science. In Horwich, P. (Ed). 1993. World changes. T.S. Kuhn and the nature of science. Cambridge: MIT Press: 55 - 78.

Montague, R. M. 1962. Deterministic theories. In Washburne, N. F. (Ed). 1962. Decisions, values, and groups. Oxford: Pergamon Press.

Morgan, M. 1988. Finding a satisfactory empinical model. In De Marchi, N. (Ed). 1988. The Popperian legacy in economics. Cambridge: Cambridge University Press: 199 - 211.

Moulines, C.U. 1991. Pragmatics in the structuralist view of science. In Schurz, G. \& G. J. W. Dom. (Eds). 1991. Advances in scientific philosophy. Essays in honour of Paul Weingartner. Amsterdam: Rodopi: $313-326$.

Musgrave, A. 1981. On interpreting Friedman, KYKLOS, (34): 377 - 387.

Nagel, E. 1961. The structure of science. London: Routledge \& Kegan Paul.

Nersessian, N.J. 1984. Faraday to Einstein: Constructing meaning in scientific theories. Dordrecht: Martinus Nijhoff Publishers.

Newton, I. 1952. (Based on the $4^{\text {th }}$ ed., London, 1730.) Opticks, or, A treatise of the 
reflections, refractions, inflections, and colours of light. New York: Dover Publications.

Niiniluoto, I. 1987, Truthlikeness. Dordrecht: Reidel.

Niiniluoto, I. 1998. Verisimilitude: The third period. (Survey Article). The Eritish joumal for the philosophy of science, Vol. 49: 1 - 29.

Norris, C. 1997. Against relativism. Philosophy of science, deconstruction and critical theory. Oxford: Blackwell.

Oddie, G. 1986. Likeness to the truth. Dordrecht: Reidel.

Papineau, D. 1995. Philosophy of science. In Honderich, T. (Ed). 1995. The Oxford companion to philosophy. New York: Oxford University Press: 809 - 812.

Pearce, D. \& V. Rantala. 1983. New foundations for metascience. Synthese, Vol. 56: 1 - 26.

Penrose, R. 1997. The mysteries of quantum physics. In Penrose, R. (Ed). 1997. The large, the small and the human mind. Cambridge: Cambridge University Press: 50 - 92.

Popper, K.R. 1989. (5 $5^{\text {th }}$ ed. revised) Conjectures and refulations. The growth of scientific knowiedge. London: Routledge.

Popper, K.R. 1990. A world of propensities. Bristol: Thoemmes.

Przelewski, M. 1969. The logic of empincal theories. London: Routledge \& Kegan Paul.

Przelewski, M. 1974. On a model-theoretic approach to empirical interpretation of scientific theories. Synthese, Vol. 26: 401- 406.

Przelewski, M. \& R. Wójcicki. 1969. The problem of analiticity. Synthese, Vol. 18: 374 - 399.

Putnam, H. 1962. What theories are not. In Nagel, E., P. Suppes \& A. Tarski. (Eds). 1962. Logic, methodology, and philosophy of science: Proceedings of the 1960 intemational congress. Stanford: Stanford University Press.

Putnam, H. 1975. Mathematics, matter and methodology. Cambridge: Cambridge University Press.

Putnam. H. 1978. Realism and reason. In Putnam H. 1978. Meaning and the moral sciences. Boston: Routledge \& Kegan Paul.

Rantala, V. 1978. The old and the new logic of metascience. Synthese, Vol. 39: $233-247$.

Rantala, V. 1980. On the logical basis of the structuralist philosophy of science. Enkenntnis, Vol. 15: $269-286$.

Redhead, M. L. G. 1980. Models in physics. The Eritish joumal for the philosophy of science, Vol. 31: 145 - 163.

Robinson, A. 1986. ( $2^{\text {nd }}$ ed.) Introduction to madel-theory and the metamathematics of algebra. New York: North Holland Publishers.

Rubin, H. \& P. Suppes. 1954. Transformations of systems of relativistic particle mechanics. Pacific joumal of mathematics, 4: 563 - 601. 
Rueger, A. \& W.D. Sharp. 1996. Simple theories of a messy world: Truth and explanatory power in nonlinear dynamics. The British joumal for the philosophy of science, (47): $93-112$.

Ruttkamp, E.B. 1997a. The role of models in philosophy of science: Mediating between the "general" and the "particular". In Forrai, G. (Ed). 1997. Images and reality. Proceedings of the 1996 Miskolc conference. Miskolc: Miskolci Egyetem Tár; 127 - 138.

Ruttkamp, E. B. 1997b. A model-theoretic interpretation of science. The South African jouma/ of philosophy, Vol. 16(1): 31 - 36.

Ruttkamp, E.B. 1999a. Semantic approaches in the philosophy of science. The South African joumal of philosophy, Vol. 18(2). (Forthcoming.)

Ruttkamp, E.B. 1999b. Reality in science. The South African joumal of philosophy, Vol. 18(2). (Forthcoming.)

Ryan, M. \& P.-Y, Schobbens. 1995. Belief revision and venisimilitude. The Notre Dame joumal of formal logic, Vol. 36: 15 - 29.

Sachs, M. 1988. Einstein versus Bohr. Illinois: Open Court Publishing Company.

Sarlemijn, A. \& M. Spamaay. (Eds). 1989. Physics in the making. Essays on developments in 20th century physics. In honour of H.B.G. Casimir. New York: North Holland Publishers.

Sartori, L. 1996. Understanding relativity. A simplified approach to Einstein's theories. Berkeley: Califomia University Press.

Scott, D. \& P. Suppes. 1958. Foundational aspects of theories of measurement. The joumal of symbolic logic, Vol. 23: $113-128$.

Schwinger, J, 1986. Einstein's legacy. New York: Scientific American Books.

Sellars, W. 1957. Counterfactuals, dispositions, and the causal modalities. In Minnesota studies in the philosophy of science, Vol II: 225 - 308.

Sellars, W. 1963. Science, perception, and reality. New York: Humenities Press.

Sneed, J.D. 1976. Philosophical problems in the empirical science of science: a formal approach. Erkenntnis, Vol. 10: 115 - 146.

Sneed, J.D. 1979. (2 $2^{\text {nd }}$ revised ed.) The logical structure of mathematical physics. Dordrecht: D. Reidel.

Sneed, J.D. 1983. Structuralism and scientific realism. Erkenntnis, Vol. 19: 245 - 370.

Sneed, J. D. 1994. Structural explanalion. In Humphreys, P. (Ed). 1994. Patrick Suppes: Scientific philosopher. Volume 2, Philosophy of physics, theory structure, and measurement theory. Dordrecht: Kluwer Academic Publishers:195 - 216.

Spurrett, D. 1998. Transcendental realism defended: a response to Allan. The South African joumal of Philosophy, Vol. 17(3): 198 - 210.

Stegmüller, W. 1976. The structure and dynamics of theories. Berlin: Springer-Verlag.

Stegmüller, W. 1979. The structuralist view of theories: $A$ possible analogue of the Bourbaki programme in physica/ science. Berlin: Springer-Verlag. 
Suchting, W. 1992. Reflections upon Roy Bhaskar's "critical realism". Radical Philosophy, Vol. 61: 23-31.

Suppe, F. 1967. The meaning and uses of models in mathematics and the exact sciences. Ph.D. Thesis, University of Michigan.

Suppe, F. 1973. Theories, their formulations and the operational imperative. Synthese, Vol. 25: $129-164$.

Suppe, F. 1989. The semantic conception of theories and scientific realism. Illinois: University of Illinois Press.

Suppes, P. 1954. Some remarks on problems and methods in the philosophy of science. Philosophy of science, 21: 242 - 248.

Suppes, P. 1959. Axioms for relativistic kinematics with or without parity. In Henkin, L., P. Suppes, \& A. Tarski. (Eds). 1959. The axiomatic method. Amsterdam: North-Holland Publishers.

Suppes. P. 1960. A comparison of the meaning and uses of models in mathematics and the empirical sciences. Synthese, Vol. 12: 287 - 300.

Suppes, P. 1967. What is a scientific theory? In Morgenbesser, S. (Ed). 1967. Philosophy of science today. New York: Basic Books: 55 - 67.

Suppes, P. 1969. Studies in the methodology and the foundations of science. Dordrecht: D. Reidel \& Co.

Suppes, P. 1988a. Philosophical implications of Tarski's work. The joumal of symbolic logic, Vol. 53: 80 - 91 .

Suppes, P. 1988b. Representation theory and the analysis of structure. Philosophia Naturalis, Vol. 25: 254 - 268.

Suppes, P. 1989. Methodology: models and measurement. In Suppes, P. 1989. Studies in the methodology and foundations of science. Selected papers from 1951 to 1969. Dordrecht: D. Reidel: Part I: 1 - 80.

Suppes, P. 1993. Models and methods in the philosophy of science: Selected essays. Dordrecht: Kluwer Academic Publishers.

Tarski, A. 1935. Der Wahrheitsbegriff in den formalisierten Sprachen. Studia Philosophica, Vol. 1: 261 - 405. (English translation by J.H. Woodger, 1956. The concept of truth in formalised languages. In Logic, semantics, metamathematics. Oxford: Oxford University Press: 152 - 278.)

Torretti, R. 1990. Creative understanding: Philosophical reflections on physics. Chicago: University of Chicago Press.

Tuomela, R. 1972a. Deductive explanation of scientific laws. The journal of philosophical logic, Vol. 1: 369 - 392.

Tuomela, R. 1972b. Model theory and empirical interpretation of scientific theories. Synthese, Vol. 25: $165-175$. 
Tuomela, R. 1974. Empiricist vs realist semantics and model theory. Synthese, Vol. 26: 407 408.

Van Fraassen, B.C. 1969. Meaning relations among predicates. NOUS, Vol. 3: 155 - 167.

Van Fraassen, B.C. 1970. On the extension of Beth's semantics for physical theories. Philosophy of science, Vol. 37: 325 - 339.

Van Fraassen, B.C. 1976. To save the phenomena. The journal of philosophy, Vol. LXXIII(18): $623-632$.

Van Fraassen, B. C. 1980. The scientific image. Oxford: Oxford University Press.

Van Fraassen, B.C. 1997. Putnam's paradox: metaphysical realism revamped and evaded. In Tomberlin, J.E. (Ed). 1997. Philosophical perspectives, 11, Mind, Causation, and World, A supplement to NOÛS. Boston: Blackwell Publishers: 17 - 42.

Von Neumann, J. 1955. Mathematical foundations of quantum mechanics. Princeton: Princeton University Press.

Wartofsky, M. W. 1979. Models: Representation and understanding of science. Dordrecht: D. Reidel.

Wojcicki, R. 1979. Topics in the formal methodology of empirical sciences. Dordrecht: D. Reidel.

Wojjcicki, R. 1994. Theories and theoretical models. In Humphreys, P. (Ed). 1994. Patrick Suppes: Scientific philosopher. Volume 2, Philosophy of physics, theory structure, and measurement theory. Dordrecht: Kluwer Academic Publishers: 125 - 149.

Worrall, J. 1994. Introduction. In Worrall, J. (Ed). 1994. The ontology of science. Aldershot: Dartmouth: $x i-x x x$.

Zamora Bonilla, J.P. 1996. Verisimilitude, structuralism, and scientific progress. Erkenntnis, Vol. $44: 25$ - 48.

Zwart, S.D. 1998. Approach to the truth. Verisimilitude and truthlikeness. Amsterdam: Institute for logic, language and computation. 